JOINT TRANSPORTATION RESEARCH PROGRAM

FHWA/IN/JTRP-2007/23

Final Report

ANALYSIS OF LATERALLY LOADED PILES IN MULTILAYERED SOIL DEPOSITS

\author{
Dipanjan Basu \\ Rodrigo Salgado \\ Monica Prezzi
}

May 2008 
Final Report

FHWA/IN/JTRP-2007/23

\title{
Analysis of Laterally Loaded Piles in Multilayered Soil Deposits
}

\author{
by \\ Dipanjan Basu \\ Research Associate \\ Rodrigo Salgado \\ Professor \\ and \\ Monica Prezzi \\ Assistant Professor \\ School of Civil Engineering \\ Purdue University \\ Joint Transportation Research Program \\ Project No. C-36-36LL \\ File No. 6-14-38 \\ SPR-2630 \\ Prepared in Cooperation with the \\ Indiana Department of Transportation and the \\ U.S. Department of Transportation \\ Federal Highway Administration
}

The contents of this report reflect the views of the authors, who are responsible for the facts and the accuracy of the data presented herein. The contents do not necessarily reflect the official views or policies of the Indiana Department of Transportation or the Federal Highway Administration at the time of publication. This report does not constitute a standard, specification, or regulation.

Purdue University

West Lafayette, Indiana 47907

May 2008 
TECHNICAL REPORT STANDARD TITLE PAGE

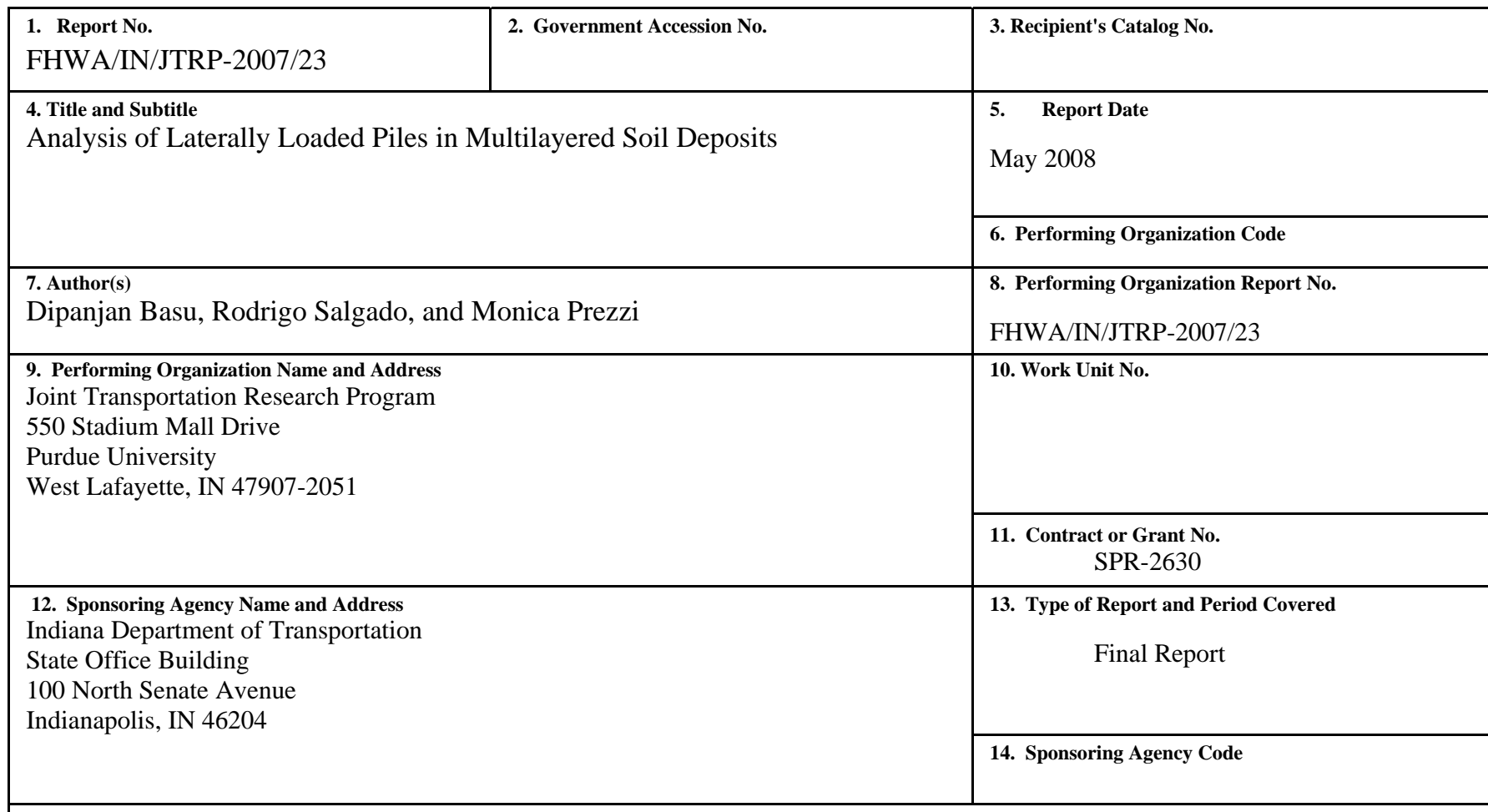

15. Supplementary Notes

Prepared in cooperation with the Indiana Department of Transportation and Federal Highway Administration.

\section{Abstract}

This report focuses on the development of a new method of analysis of laterally loaded piles embedded in a multi-layered soil deposit treated as a three-dimensional continuum. Assuming that soil behaves as a linear elastic material, the governing differential equations for the deflection of laterally loaded piles were obtained using energy principles and calculus of variations. The differential equations were solved using both the method of initial parameters and numerical techniques. Soil resistance, pile deflection, slope of the deflected pile, bending moment and shear force can be easily obtained at any depth along the entire pile length. The results of the analysis were in very good agreement with three-dimensional finite element analysis results. The analysis was further extended to account for soil nonlinearity. A few simple constitutive relationships that allow for modulus degradation with increasing strain were incorporated into the analysis. The interaction of piles in groups was also studied.

17. Key Words

laterally loaded piles, p-y method, p-y curves, soil nonlinearity, pile groups, pile design, layered soil

\section{Distribution Statement}

No restrictions. This document is available to the public through the National Technical Information Service, Springfield, VA 22161
19. Security Classif. (of this report)

Unclassified

\begin{abstract}
20. Security Classif. (of this page)
\end{abstract}
Unclassified

\begin{tabular}{|c|c|}
\hline 21. No. of Pages & 22. Price \\
149 & \\
\hline
\end{tabular}




\section{TABLE OF CONTENTS}

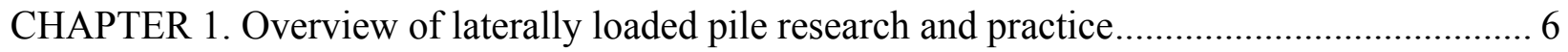

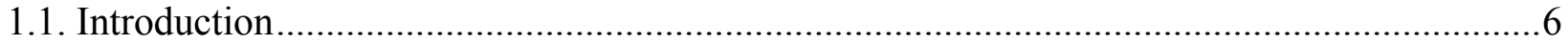

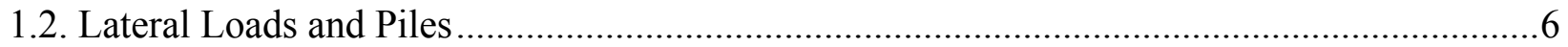

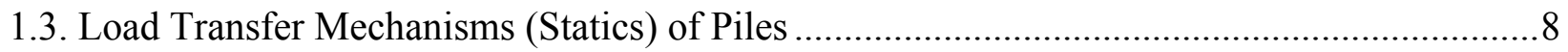

1.4. Kinematics and Failure Modes of Laterally Loaded Piles....................................................12

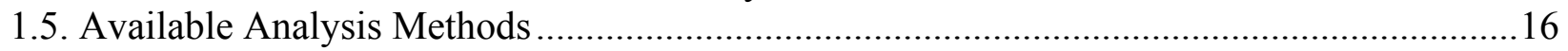

1.5.1. Beam-on-Foundation Approach ………………............................................ 16

1.5.2. Continuum Approach................................................................................... 20

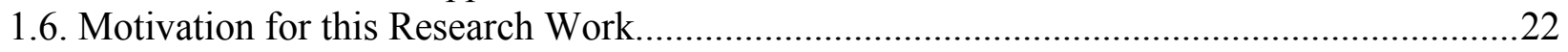

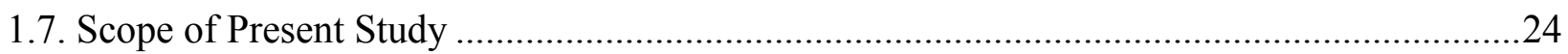

CHAPTER 2. Laterally Loaded Pile in Layered Elastic Medium: A Beam-on-Elastic-Foundation

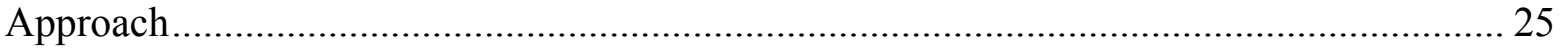

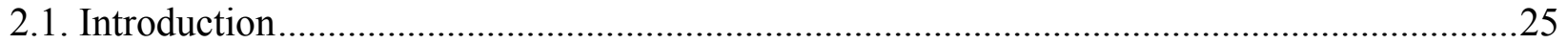

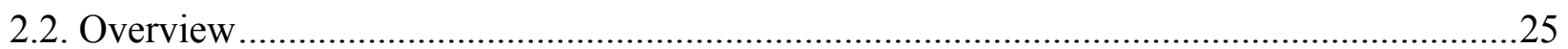

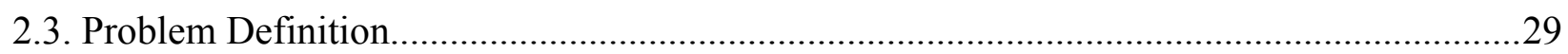

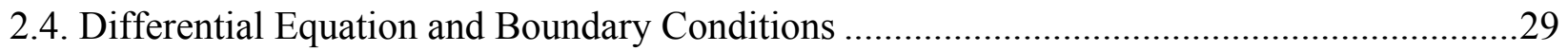

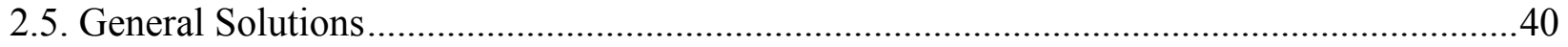

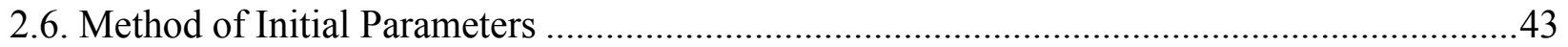

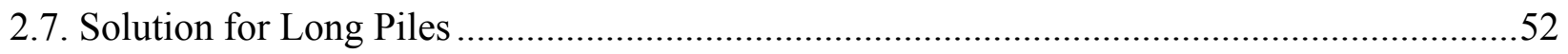

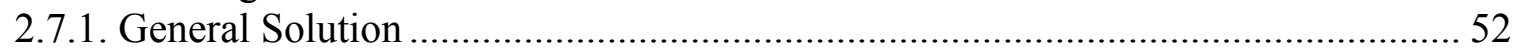

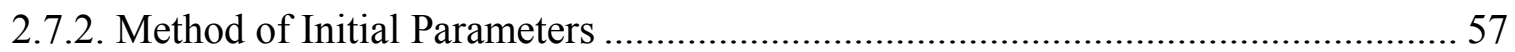

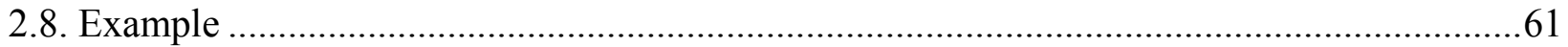

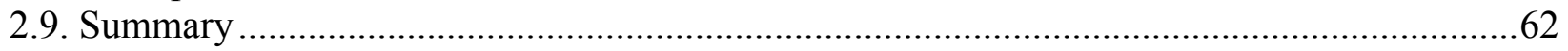

CHAPTER 3. Continuum Analysis of Laterally Loaded Pile in Layered Elastic Medium........... 64

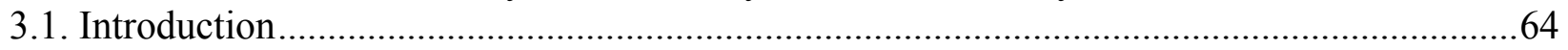

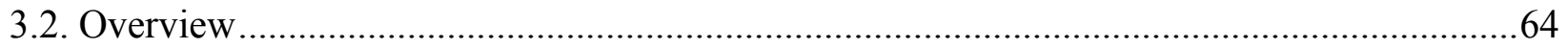

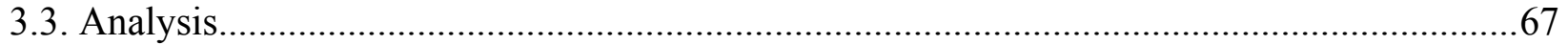

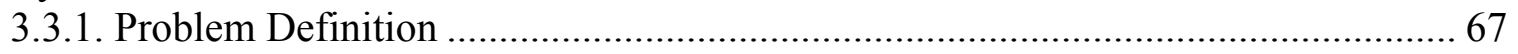

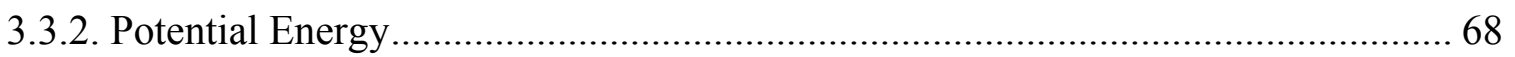

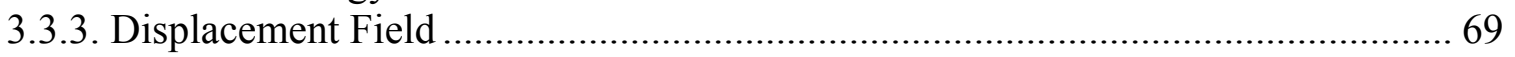

3.3.4. Stress-Strain-Displacement Relationships ........................................................... 70

3.3.5. Principle of Minimum Potential Energy ……………......................................... 71

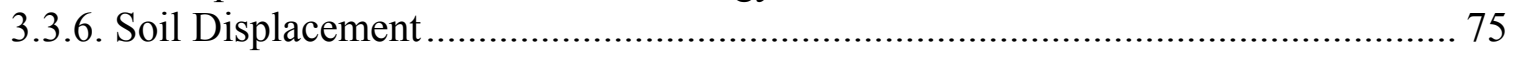

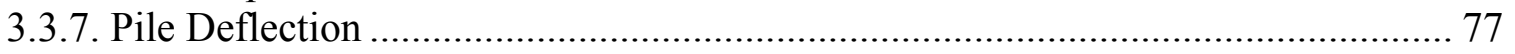

3.3.8. Expression of $\gamma$ 's in Terms of Dimensionless Deflections ..................................... 80

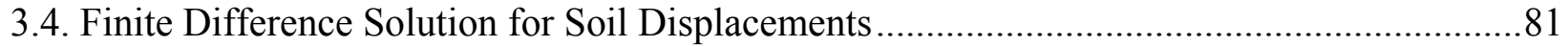

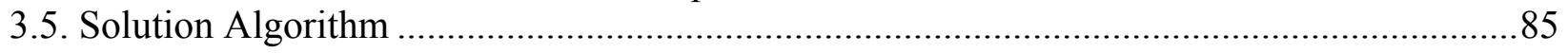




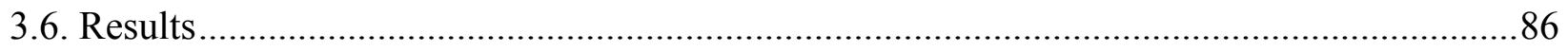

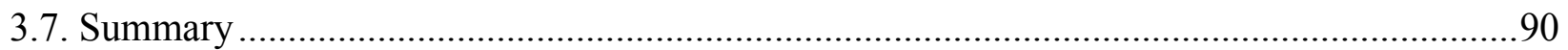

CHAPTER 4. Nonlinear Analysis of Laterally Loaded Pile in Layered Soil Medium ................. 91

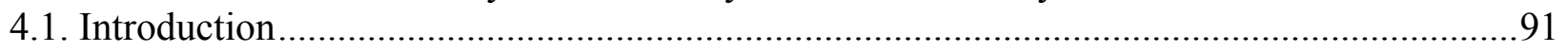

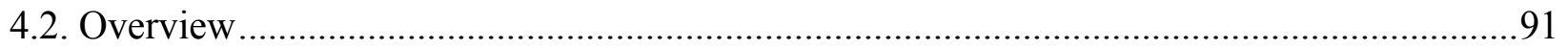

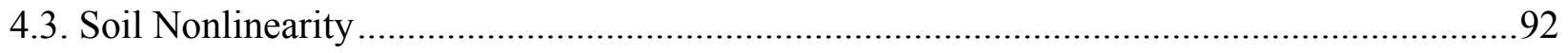

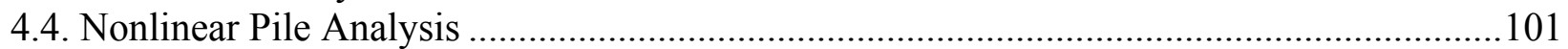

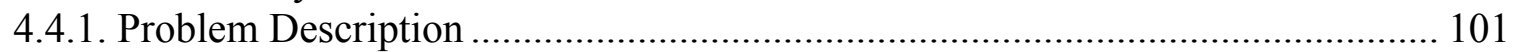

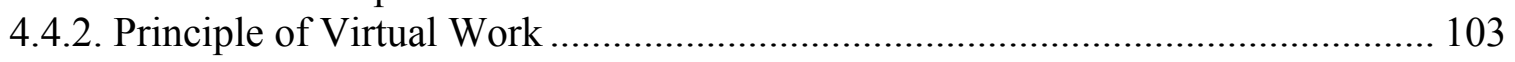

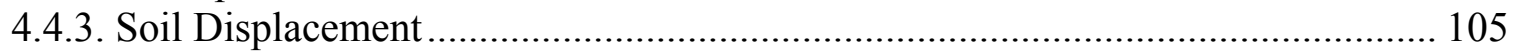

4.4.4. Finite Difference Solution for Soil Displacements............................................... 107

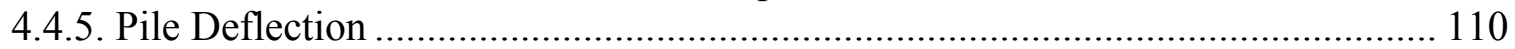

4.5. Interdependence and Iterative Solutions of Pile and Soil Displacements...............................114

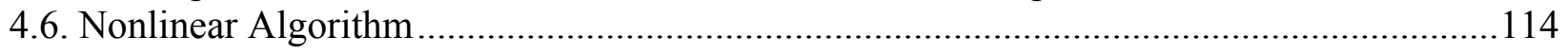

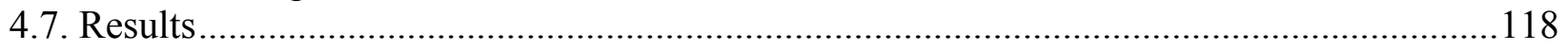

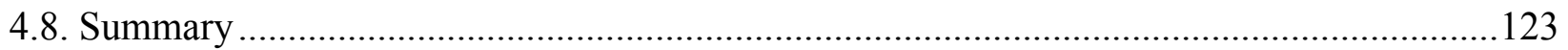

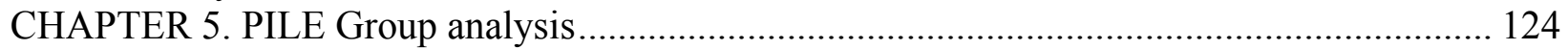

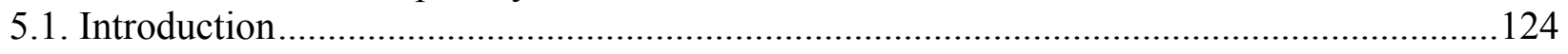

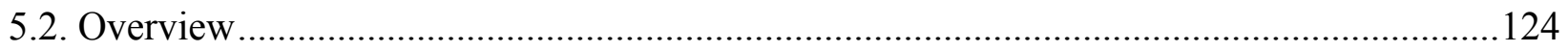

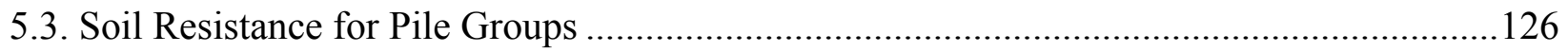

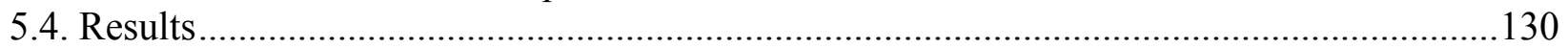

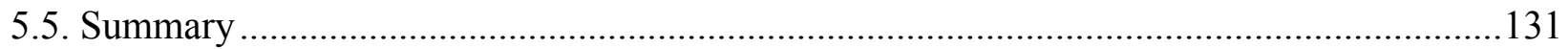

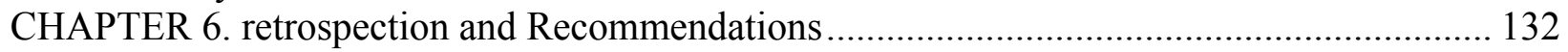

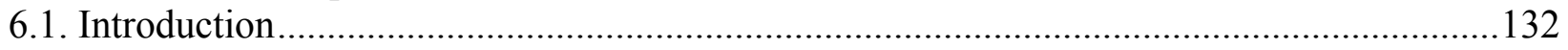

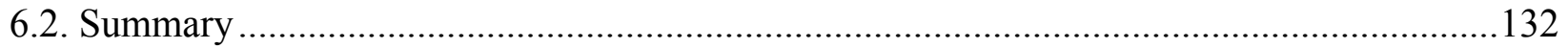

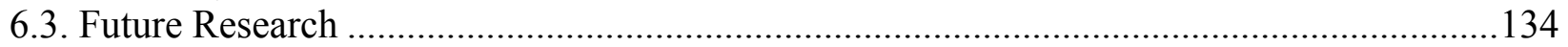

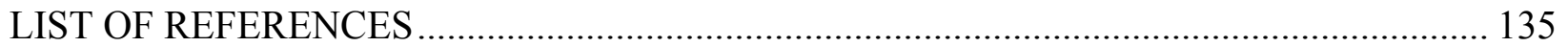




\section{LIST OF FIGURES}

Figure 1-1 Load Transfer Mechanism of Axially Loaded Piles ................................................ 9

Figure 1-2 Load Transfer Mechanism of Laterally Loaded Piles............................................ 10

Figure 1-3 Load transfer mechanism for vertically loaded pile group ...................................... 11

Figure 1-4 Illustration of overlapping zones creating additional load on piles within a group .... 12

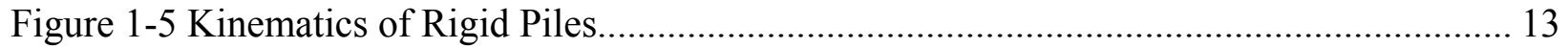

Figure 1-6 Kinematics of Flexible Piles ............................................................................... 13

Figure 1-7 Kinematics of a vertically loaded pile group ................................................... 14

Figure 1-8 Kinematics of a laterally loaded pile group ..................................................... 15

Figure 1-9 A Beam on an Elastic Foundation.......................................................................... 17

Figure 1-10 A Laterally Loaded Pile in a Bed of Springs .................................................... 18

Figure 1-11 Comparison of Pile Resistance $p$ versus Normalized Pile Deflection y/D (D is the

Pile Diameter) Curves Obtained from Model Tests with the Standard Curves Available for

Design (Adapted from Kim et al. 2004) ........................................................................... 23

Figure 2-1 (a) Deflection Predicted by One-Parameter Model; (b) Actual Deflection Profile .... 26

Figure 2-2 (a) A Laterally Loaded Pile in a Layered Soil Medium........................................... 30

Figure 2-3 Pile-Soil Interaction .................................................................................... 31

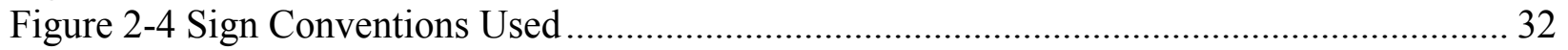

Figure 2-5 Equilibrium of Pile and Soil................................................................................ 32

Figure 2-6 A Laterally Loaded Pile in a Three-Layer Medium .............................................. 47

Figure 2-7 Piles in (a) Dense Sand and (b) Soft Clay .......................................................... 55

Figure 2-8 (a) Deflection, (b) Bending Moment, (c) Shear Force and (d) Soil Resistance of a

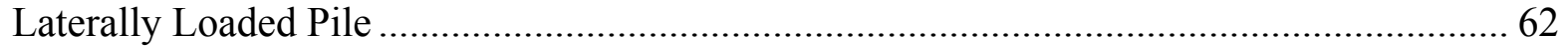

Figure 3-1 A Laterally Loaded Pile in a Layered Elastic Medium.......................................... 68

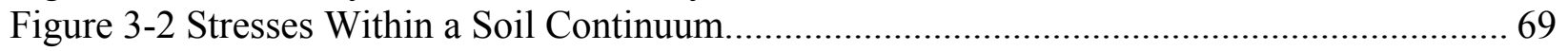

Figure 3-3 Displacements Within a Soil Continuum ...................................................... 70

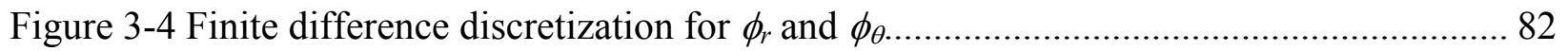

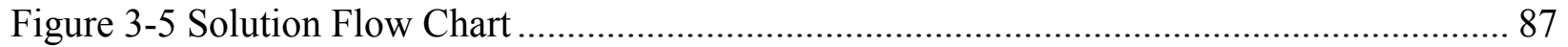

Figure 3-6 Deflection profile of a $15-\mathrm{m}$-long pile ............................................................... 88

Figure 3-7 Deflection profile of a 40-m-long drilled shaft .................................................. 88

Figure 3-8 Deflection profile for the pile load test of McClelland and Focht (1958) ................ 89

Figure 4-1 Typical Stress-Strain Plot of Soil under Drained Condition..................................... 93

Figure 4-2 Typical Modulus Degradation Curve of Soil ......................................................... 93

Figure 4-3 Hyperbolic Stress-Strain Plot of Soil ................................................................. 96

Figure 4-4 Variations of Soil Displacement, Strain and Modulus, at a given Depth $z_{0}$, with Radial

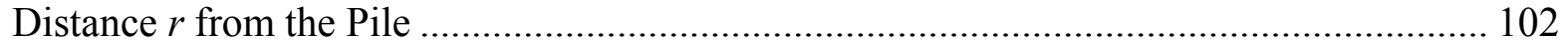

Figure 4-5 Tangential Variation of Soil Displacement and Modulus Surrounding a Pile......... 102

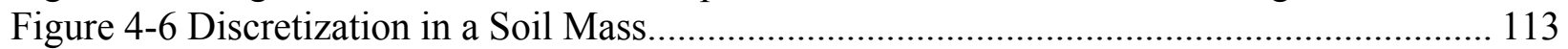

Figure 4-7 Nonlinear Solution Flow Chart ............................................................................ 117

Figure 4-8 Head Deflection as a Function of Applied Force for a Pile in Sand ....................... 119

Figure 4-9 Head Deflection as a Function of Applied Force for a Pile in Sand ....................... 120 
Figure 4-10 Soil Profile at the Pile Load Test Site in Orange County, Indiana ....................... 121

Figure 4-11 Head Deflection versus Applied Force for the Orange County Pile Load Test...... 123

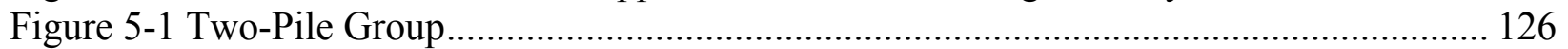

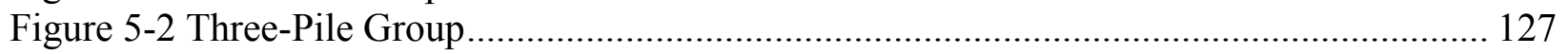

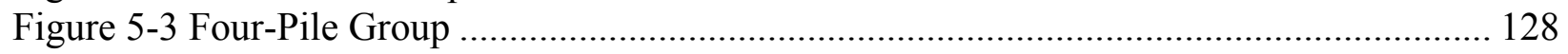

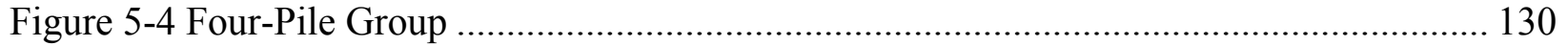

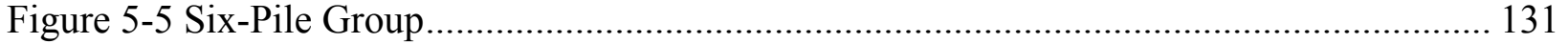




\section{LIST OF TABLES}

Table 2-1 Functions in Equation (2-40) for Piles Crossing Multiple Soil Layers. 42 Table 2-2 Functions in Equation (2-102) for Infinitely Long Piles Crossing Multiple Soil Layers

Table 3-1 Soil Properties at the Pile Load Test Site of Ismael and Klym (1978)........................ 90

Table 4-1 Soil Properties at the Pile Load Test Site in Orange County, Indiana ........................ 122 


\section{CHAPTER 1. OVERVIEW OF LATERALLY LOADED PILE RESEARCH AND PRACTICE}

\subsection{Introduction}

The report documents the development of a new method of analysis of laterally loaded piles. The prevalent method of analysis in the U.S., namely the p-y method, often fails to predict pile response (Kim et al. 2004, Anderson et al. 2003). This is not surprising because the p-y curves, which describe the resistive properties of soil as a function of pile deflection, used in the p-y analysis are developed empirically by back-fitting the results of numerical analysis to match the actual field pile-load test results. Thus, p-y curves developed for a particular site are not applicable to other sites. In order to obtain an accurate prediction of lateral pile response by the p-y method, p-y curves must be developed through pile load tests for every site. Since a pile load test at every site is not feasible economically, an alternative method of analysis is required.

A method of laterally loaded pile analysis is developed that takes into account the physics behind the complex three-dimensional pile-soil interaction. The method rationally relates the elemental resistive properties of soil to the overall resistance of the ground against lateral pile movement. Since the physics of the resistive mechanism is captured, no site specific calibration is necessary for this method. The inputs required for the analysis are simple soil parameters that an engineer can determine in the field without much difficulty.

In this chapter, we provide a general overview of laterally loaded piles and pile groups. We explain why lateral loads act on piles and how piles interact with the surrounding ground as a result of those lateral loads. We then examine the available methods of analysis of laterally loaded piles, discuss where improvements are necessary and point out scope of this research.

\subsection{Lateral Loads and Piles}

Piles are commonly used to transfer vertical (axial) forces, arising primarily from gravity (e.g., the weight of a superstructure). Examples of structures where piles are commonly used as 
foundations are tall buildings, bridges, offshore platforms, defense structures, dams and lock structures, transmission towers, earth retaining structures, wharfs and jetties. However, in all these structures, it is not only the axial force that the piles carry; often the piles are subjected to lateral (horizontal) forces and moments. In fact, there are some structures (e.g., oil production platforms, earth retaining structures, wharfs and jetties) where the primary function of piles is to transfer lateral loads to the ground.

Wind gusts are the most common cause of lateral force (and/or moment) that a pile has to support. The other major cause of lateral force is seismic activity. The horizontal shaking of the ground during earthquakes generates lateral forces that the piles have to withstand. Certain buildings are also acted upon by lateral earth pressures, which transmit lateral forces to the foundations. That apart, depending on the type of structure a pile supports, there can be different causes of lateral forces. For tall buildings and transmission towers, wind action is the primary cause. For offshore oil production platforms, quays, harbors, wharfs and jetties, wave action gives rise to lateral forces. In the case of bridge abutments and piers, horizontal forces are caused due to traffic and wind movement. Dams and lock structures have to withstand water pressures which transfer as horizontal forces on the supporting piles. Defense structures often have to withstand blasts that cause lateral forces. In the case of earth retaining structures, the primary role of piles is to resist lateral forces caused due to the lateral pressures exerted by the soil mass behind the retaining wall. Sometimes, piles are installed into slopes, where slow ground movements are taking place, in order to arrest the movement. In such cases, the piles are subjected only to lateral forces. Piles are used to support open excavations; here also, there is no axial force and the only role of the piles is to resist lateral forces.

In the above examples, there are some cases in which the external horizontal loads act at the pile head (i.e., at the top section of the pile). Such loading is called active loading (Fleming et al. 1992, Reese and Van Impe 2001). Common examples are lateral loads (and moments) transmitted to the pile from superstructures like buildings, bridges and offshore platforms. Sometimes the applied horizontal load acts in a distributed way over a part of the pile shaft; such a loading is called passive loading. Examples of passive loading are loads acting on piles due to movement of slopes or on piles supporting open excavations. There are cases in which external horizontal loads are minimal or absent; even then external moments often exist because of load eccentricities caused by construction defects, e.g., out-of-plumb constructions. Thus, piles in 
most cases are subjected to lateral loads. Consequently, proper analysis of laterally loaded piles is very important to the geotechnical and civil engineering profession.

\subsection{Load Transfer Mechanisms (Statics) of Piles}

A proper understanding of the load transfer mechanisms for piles is necessary for analysis and design. Piles transfer axial and lateral loads through different mechanisms. In the case of axial (vertical) loads, piles may be looked upon as axially loaded columns; they transfer loads to the ground by shaft friction and base resistance (Figure 1-1) (Salgado 2008). As a pile is loaded axially, it slightly settles and the surrounding soil mass offers resistance to the downward movement. Because soil is a frictional material, frictional forces develop at the interface of the pile shaft and the surrounding soil that oppose the downward pile movement. The frictional forces acting all along the pile shaft partly resist the applied axial load and are referred to as shaft resistance, shaft friction or skin friction. A part of the axial load is transferred to the ground through the bottom of the pile (commonly referred to as the pile base). As a pile tries to move down, the soil mass below the pile base offers compressive resistance to the movement. This mechanism is called base resistance or end-bearing resistance. The total resistance (shaft friction plus end-bearing resistance) keeps a pile in equilibrium with the applied load. Piles that transfer most of the axial load through the base are called end-bearing piles, while those that transfer most of the load through shaft friction are called friction piles. For end-bearing piles, it is necessary to have the pile base inserted into a strong layer of soil (e.g., dense sand, stiff clay or rock). Typically, engineers would prefer to design end-bearing piles because the base resistance is more reliable than shaft friction. However, if no such strong layer is available at a site, then engineers have to rely only on shaft friction; in such a case the pile is called a floating pile. 


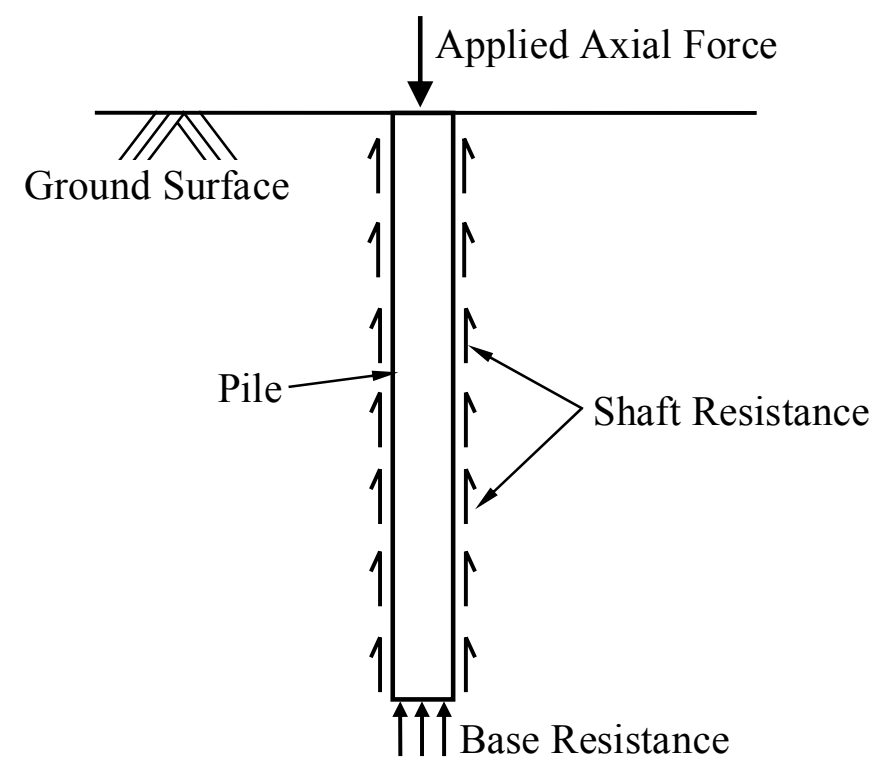

Figure 1-1 Load Transfer Mechanism of Axially Loaded Piles

In the case of lateral loads, piles behave as transversely loaded beams. They transfer lateral load to the surrounding soil mass by using the lateral resistance of soil (Figure 1-2). When a pile is loaded laterally, a part or whole of the pile tries to shift horizontally in the direction of the applied load, causing bending, rotation or translation of the pile (Fleming et al. 1992, Salgado 2008). The pile presses against the soil in front of it (i.e., the soil mass lying in the direction of the applied load), generating compressive and shear stresses and strains in the soil that offers resistance to the pile movement. This is the primary mechanism of load transfer for lateral loads. The total soil resistance acting over the entire pile shaft balances the external horizontal forces. The soil resistance also allows satisfaction of moment equilibrium of the pile. 


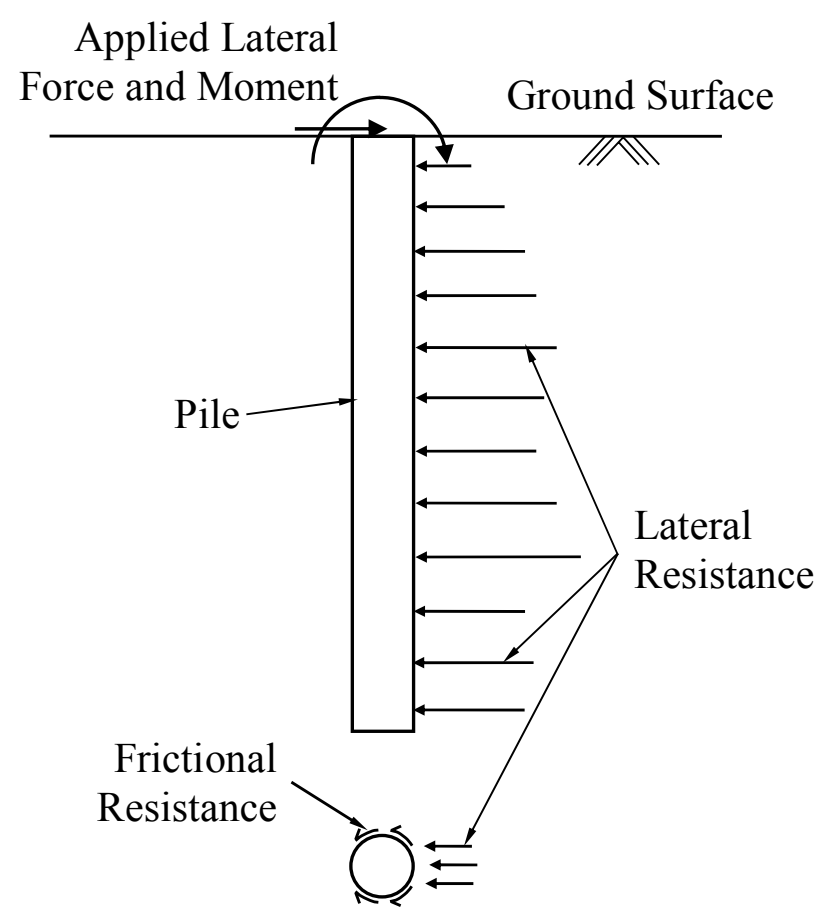

Figure 1-2 Load Transfer Mechanism of Laterally Loaded Piles

Often, the load acting on a superstructure is larger than the capacity of a single pile. When that happened, piles are grouped under each column to resist the total force acting at the column base. The piles in a group no longer behave as isolated units but interact with each other and resist the external load in an integrated manner. Consequently, the response of a single pile differs from that of a pile placed within a pile group (Prakash and Sharma 1990, McVay 1998., Ilyas et al. 2004, Bogard and Matlock 1983, Ashour et al. 2004). Each pile in a group, whether loaded axially or laterally, generates a displacement field of its own around itself. The displacement field of each pile interferes and overlaps with those of the adjacent piles; this results in the interaction between piles.

Similarly to single piles, pile groups have two resistance mechanisms against vertical loads: friction along the sides and base resistance. However, compared with the behavior of an isolated pile, the response of a pile within a group differs due to the interaction of the adjacent piles. The difference in response is more pronounced for pile groups that resist vertical loads primarily by side friction (Figure 1-3). Additional forces are induced along the pile shafts due to the settlement of adjacent piles. Thus, the piles resist not only the vertical column load but also 
the interaction forces along the pile shafts. For end bearing piles, however, a larger fraction of the applied load is supported by the compressive resistance of the ground below the pile base because of which the interaction along the pile shafts is minimal. Consequently, the response of each pile within a group is closer to that of a single isolated pile.

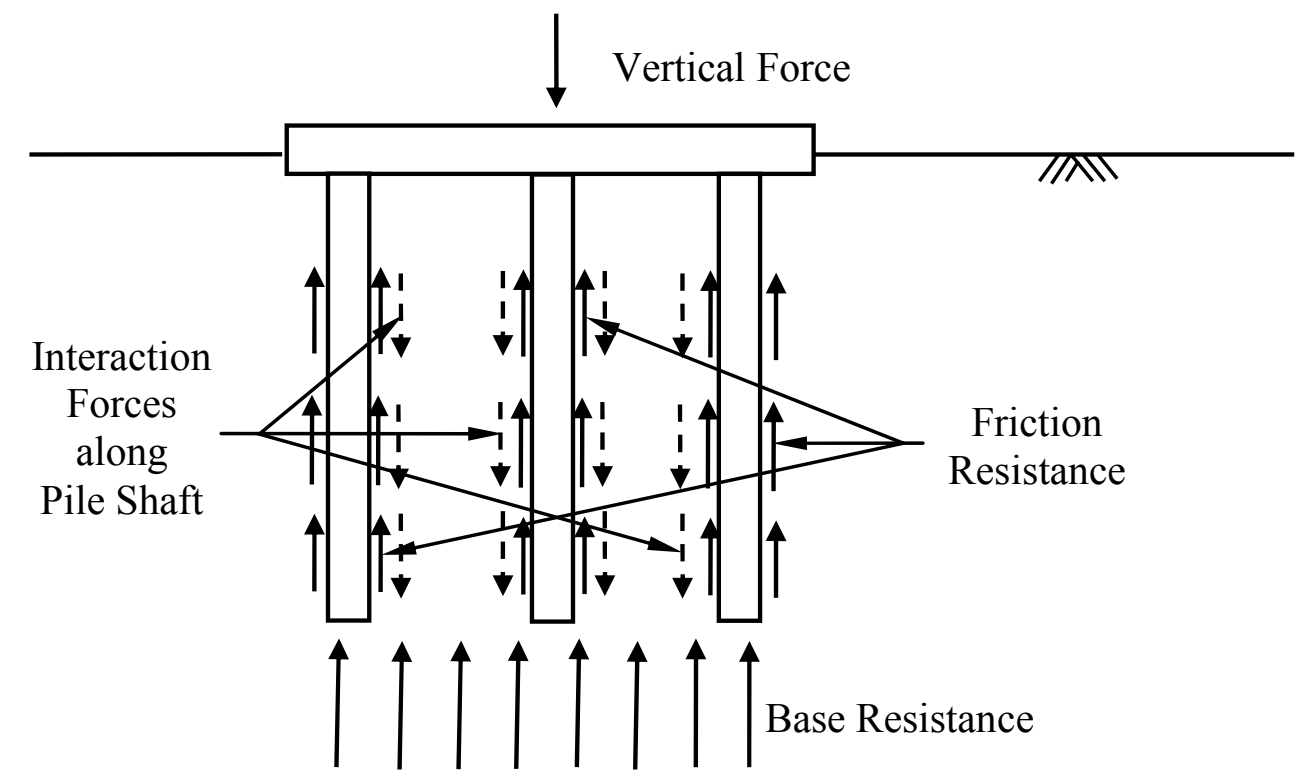

Figure 1-3 Load transfer mechanism for vertically loaded pile group

Interaction between piles occurs in the case of laterally loaded pile groups as well. In a laterally loaded pile group, each pile pushes the soil in front of it (i.e., in the direction of the applied force). Movement of the piles placed in the first (leading) row in the direction of the applied force is resisted by the soil in front of it. In contrast, the piles in the rows behind the first row (i.e., the piles in the trailing rows) push on the soil which in turn pushed on the piles in the rows in front of them (Figure 1-4). The resistive forces acting on the trailing-row piles are in general less than the resistive forces acting on the leading row (Prakash and Sharma 1990, Salgado 2008, Ilyas et al. 2004, Ashour et al. 2004). 


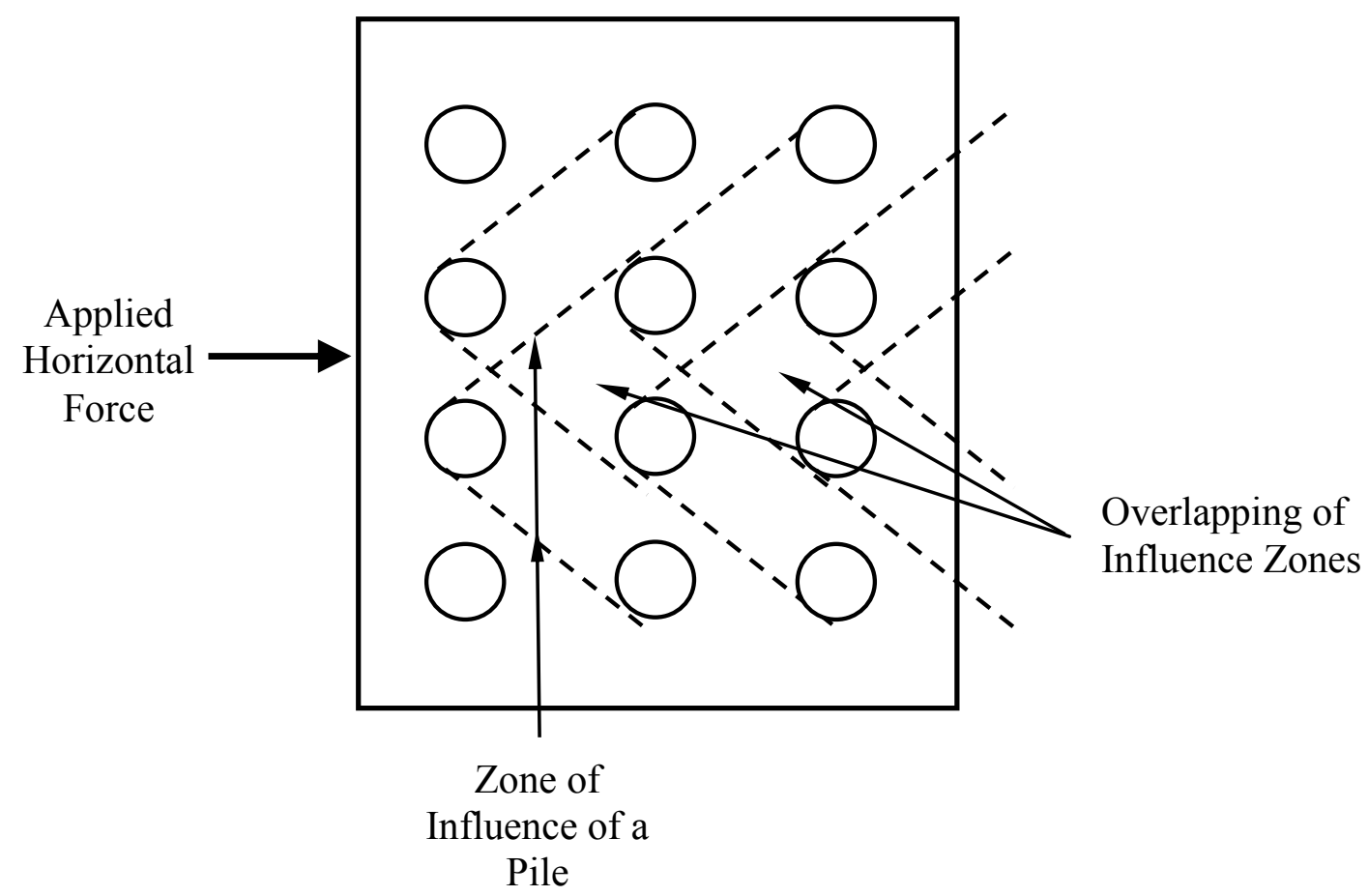

Figure 1-4 Illustration of overlapping zones creating additional load on piles within a group

\subsection{Kinematics and Failure Modes of Laterally Loaded Piles}

The kinematics of axially loaded piles is simple: the pile moves vertically downward under the acting load and, if the resistive forces (i.e., shaft and base resistances) exceed the limit values, then the pile suffers excessive vertical deflection (plunging) leading to collapse. The kinematics and failure mechanisms of laterally loaded piles are more complex and vary depending on the type of pile.

Since laterally loaded piles are transversely loaded, the pile may rotate, bend or translate (Fleming et al. 1992, Salgado 2008). As the pile moves in the direction of the applied force, a gap may also open up between the back of the pile and the soil over the top few meters. If the pile is short and stubby, it will not bend much but will rotate or even translate (Figure 1-5). Such piles are called rigid piles. If the pile is long and slender, then it bends because of the applied load (Figure 1-6). These piles are called flexible piles. In most practical situations, piles are long enough to behave as flexible piles. For flexible piles, the laterally loaded pile problem is one of soil-structure interaction; i.e., the lateral deflection of the pile depends on the soil resistance, and the resistance of the soil, in turn, depends on the pile deflection. 


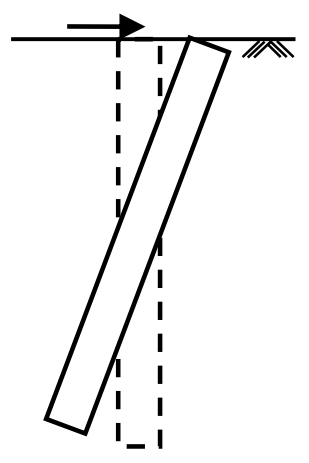

Rotation

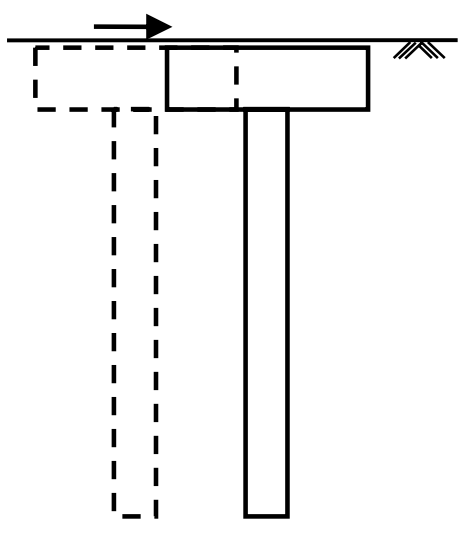

Translation

Figure 1-5 Kinematics of Rigid Piles
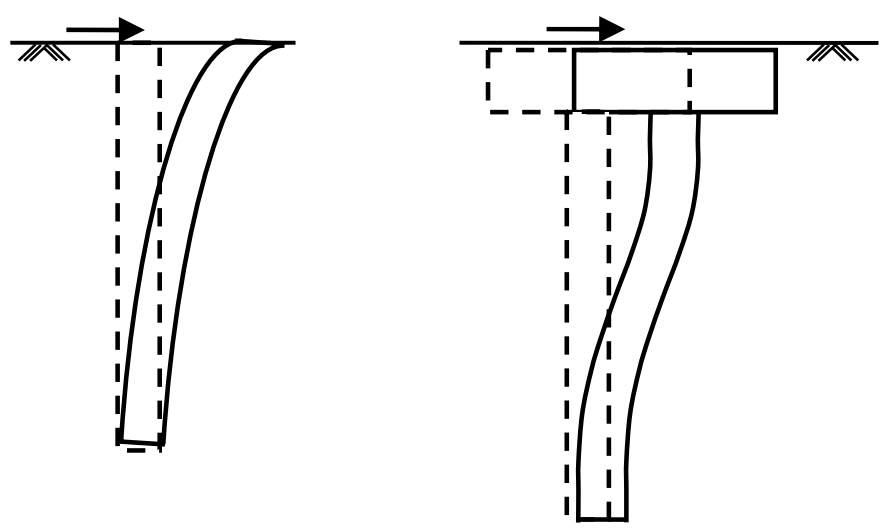

Figure 1-6 Kinematics of Flexible Piles

The kinematics of a vertically loaded pile group is similar to that of an axially loaded pile. A vertically loaded pile group moves down under the applied load. However, the difference in the response of a pile in a group and a similarly loaded isolated pile is that the pile in a group undergoes more settlement due to the additional downward forces acting on it due to the interaction of the adjacent piles (Figure 1-7) (Fleming and Randolph 1985, Salgado 2008). 


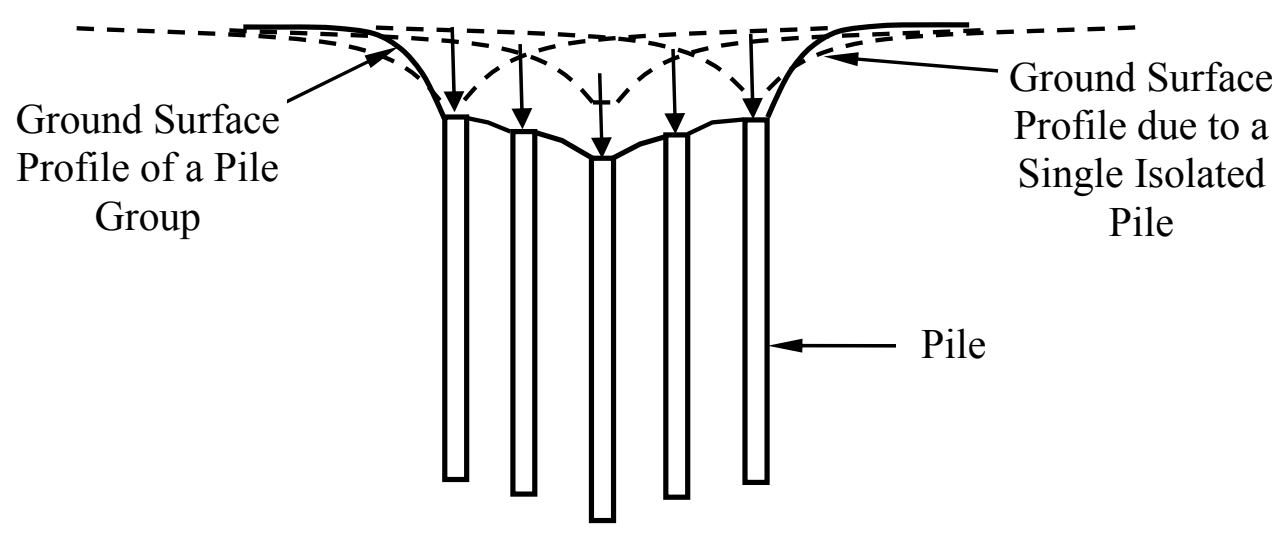

Figure 1-7 Kinematics of a vertically loaded pile group

The kinematics of a laterally pile group is such that the piles in a group may have vertical movement in addition to lateral movement, rotation and bending. If, due to the externally applied force and moment, the pile cap rotates, then the piles in the rows in front of the pile-cap center undergo downward movement while those behind undergo uplift (Figure 1-8) (Fleming and Randolph 1985, Salgado 2008). However, if the rotation of the pile cap is not large, then the piles can be assumed to move only in the horizontal direction.

Failure is a term that we engineers define for our convenience. We set some criteria which we want a structure or a foundation to satisfy. If one or more of those criteria are not satisfied, we say that the structure or the foundation has failed. In general, we identify two classes of criteria: (1) ultimate limit states and (2) serviceability limit states (Salgado 2008). Ultimate limit states are associated with dangerous outcomes, such as partial or total collapse of a structure. Serviceability limit states are used as measures to maintain the serviceability of a structure. In general, serviceability limit states refer to tolerable settlements or deflections. For design, all the possible ultimate and serviceability limit states associated with a particular structural or foundation element are identified and then it is designed so that all the limit states are satisfied. 


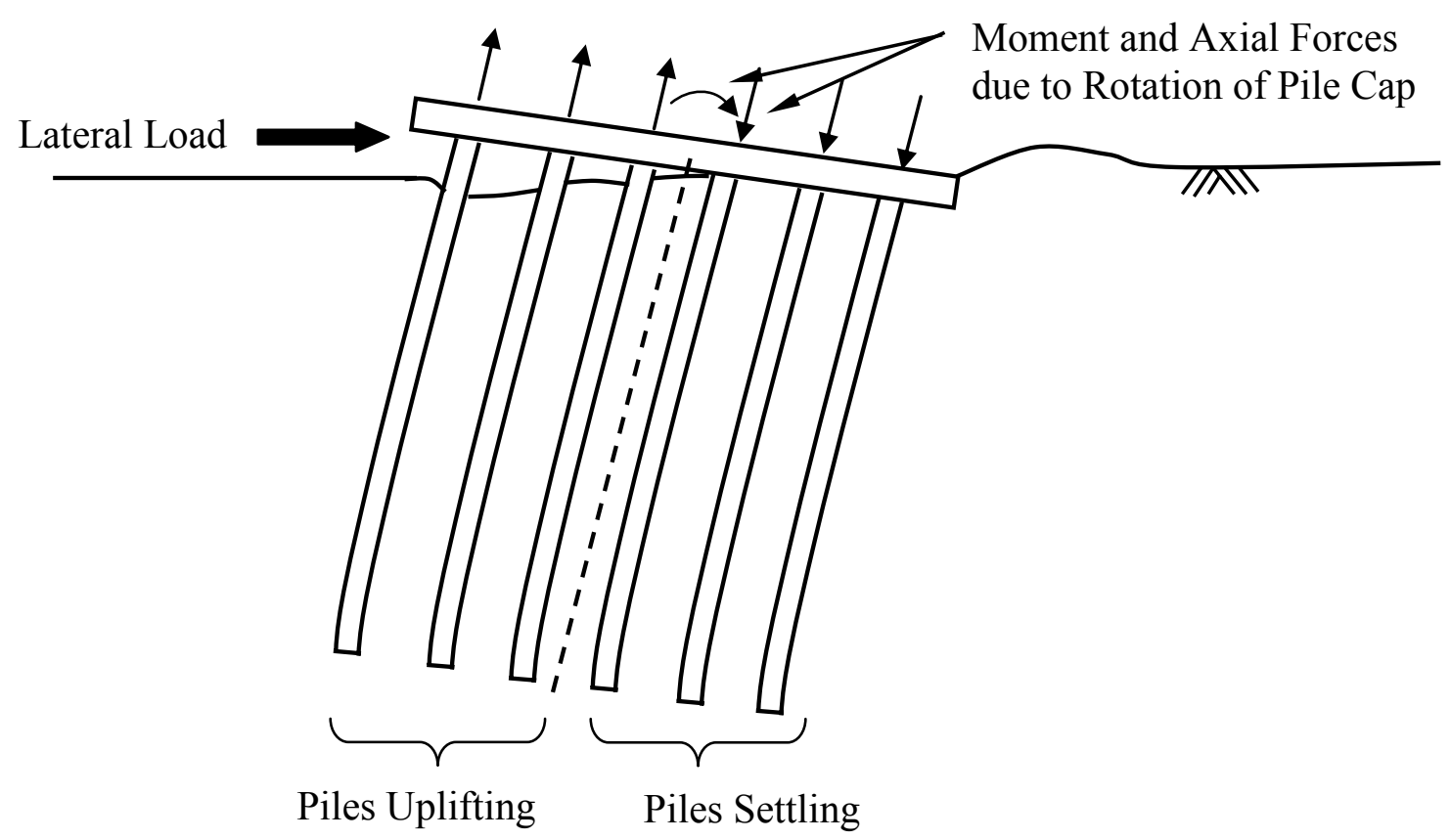

Figure 1-8 Kinematics of a laterally loaded pile group

One ultimate limit state for a laterally loaded piles is reached if the resistive stresses in the soil attain the limit (yield) value over a substantial portion of the pile length so that plastic flow occurs within the soil mass resulting in large lateral deflections, translation or rotation of the pile (e.g., inflexible piles, with possible yield or breakage of the pile at one or more cross sections). This ultimate limit state may lead to collapse of the superstructure. For flexible piles, the mechanism consists of a plastic wedge of soil that forms in front of the pile, leading to excessive lateral deflection and bending. If the bending moment is excessive, plastic hinges may form, leading possibly to collapse. Much before this pile-centered ultimate limit state is reached, other ultimate limit states or serviceability limit states may occur as the pile head deflection exceeds the tolerable head deflection. Hence, it is the restriction of the horizontal pile deflection that determines the limits of pile performance and designs in most cases. In fact, in most cases, piles are first designed against ultimate limit states corresponding to axial loads (i.e., against the limit vertical load carrying capacity) and then checked against serviceability limit states corresponding to axial and lateral loads (i.e., against vertical and lateral deflections). 
In the case of laterally loaded pile groups, a serviceability limit state restricting the lateral deflection would govern the design in most cases. However, checks against ultimate limit states resulting from the yielding of soil in front of pile rows (as well as the limit states due to formation of plastic hinges in the piles) may also be required. Additionally, checks might be necessary against the limit states arising due to the rotation of the pile cap and the associated vertical movement of the piles.

\subsection{Available Analysis Methods}

Having assessed the statics, kinematics and the possible failure modes of laterally loaded piles, we now discuss the methods available for analyzing them so that safe designs can be produced. We restrict our discussion to only piles with active loading. In fact, most of the analyses available in the literature are developed for active loading, although most of the methods can be extended to passive loading as well.

Research on analysis of laterally loaded piles started more than five decades ago. As a consequence of such sustained research, we have a number of analysis methods that can be used for design (an account of the salient analysis methods available can be obtained from Poulos and Davis 1980, Scott 1981, Fleming et al. 1992, Reese and Van Impe 2001, Reese et al. 2006). Broadly, the methods of analysis can be classified into two: 1) beam-on-foundation approach and 2) continuum approach.

\subsubsection{Beam-on-Foundation Approach}

Long before the research on laterally loaded pile started, foundation engineers had looked into the possibility of representing shallow foundations that are long and flexible enough (e.g., strip footings) as beams resting on foundations. In the context of beam-on-foundation approach, the beam represents the foundation (e.g., footings, piles etc.) and the foundation represents the soil mass. As early as 1867 , Winkler (1867) proposed that the vertical resistance of a subgrade against external forces can be assumed to be proportional to the ground deflection. Researchers, extending the idea, represented the ground with a series of elastic springs so that the compression (or extension) of the spring (which is the same as the deflection of the ground) is proportional to 
the applied load. The spring constant represents the stiffness of the ground (foundation) against the applied loads.

This concept was extended by placing an Euler-Bernoulli beam on top of the elastic foundation and applying loads on top of the beam (Figure 1-9). A differential equation governing the beam deflection for such a beam-foundation system was developed (which is a fourth order linear differential equation) and analytical solutions for different types and positions of loads and load distributions were obtained (Biot 1937, Hetényi 1946). The input parameters required are the elastic modulus and geometry of the beam, the spring constant of the foundation (soil) and the magnitude and distribution of the applied load. As a result of the analysis, the beam deflection, bending moment and shear force along the span of the beam can be determined.

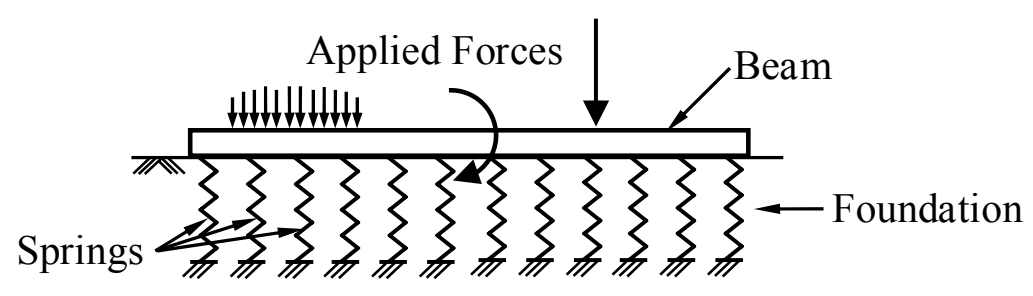

Figure 1-9 A Beam on an Elastic Foundation

It is important to mention here that there is a subtle difference between the foundation springs and the conventional springs. In conventional springs, the spring constant multiplied by the spring deflection gives the spring force. In foundation springs, the spring constant multiplied by the spring deflection (which is the same as the beam deflection) produces the resistive force of the foundation (ground) per unit beam length. Therefore, the spring constant unit for a foundation in which the resistance is expressed per unit of length is $\mathrm{FL}^{-2}$ ( $\mathrm{F}=$ force, $\mathrm{L}=$ length), while the spring constant unit of a conventional spring is $\mathrm{FL}^{-1}$.

The beam-on-foundation approach can also be called subgrade-reaction approach because the foundation spring constant can be related to the modulus of subgrade reaction of a soil mass (Terzaghi 1955, Bowles 1997) (if the pressure at a point on the contact surface between the foundation and the beam is $p$ and if, because of $p$, the deflection of the point is $\delta$, then the 
modulus of subgrade reaction is given by $p / \delta$ and has a unit of $\mathrm{FL}^{-3}$ ). The modulus of subgrade reaction multiplied by the width of the beam gives the foundation spring constant. In fact, the spring constants are often estimated by determining the soil subgrade reaction modulus (the modulus can be determined experimentally, e.g., by performing a plate load test).

The beam-on-foundation concept was adapted by the researchers on laterally loaded piles (Davisson 1970, Francis 1964, Broms 1964a, b, Matlock and Reese 1960, Reese and Matlock 1956) because, in most cases, the piles behave as flexible beams against lateral (transverse) loads and the problem can be looked upon as a beam-on-foundation problem rotated by $90^{\circ}$ (Figure 110). However, the laterally loaded pile problem is more complex because soils in real field situations behave nonlinearly, particularly near the top part of the pile. In other words, because of the nonlinear nature of a typical soil stress-strain plot, the head deflection of piles, when plotted against applied load, produce a nonlinear curve. The linear springs, as hypothesized by Winkler (1867), could no longer be used for laterally loaded piles, and were replaced by nonlinear springs (for which the value of the spring constant changes with pile deflection). As a result, the governing fourth order differential equation becomes nonlinear and the finite difference method was used to iteratively solve the equation (McClelland and Focht 1958). In order to simplify the problem, some researchers assumed the soil to be linear elastic up to a certain value of pile deflection and perfectly plastic beyond that value (Bowles 1997, Hsiung and Chen 1997).

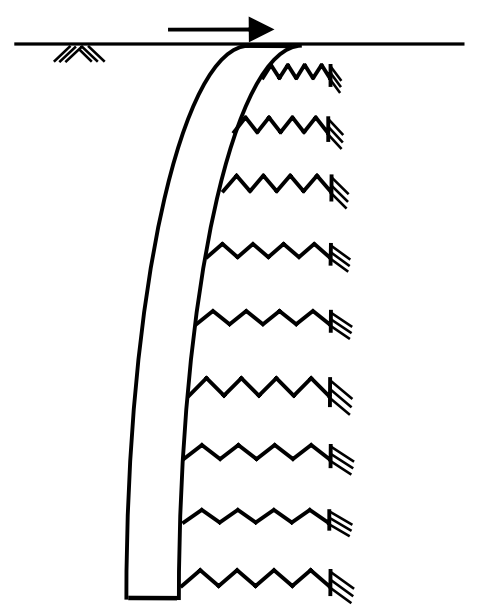

Figure 1-10 A Laterally Loaded Pile in a Bed of Springs 
Further modification of the beam-on-nonlinear-foundation approach led to the $\mathrm{p}-\mathrm{y}$ method (Matlock 1970, Reese et al. 1974, 1975, Reese and Welch 1975, Reese 1977, 1997, O'neill et al. 1990). In the p-y method, p stands for the soil pressure (resistance) per unit pile length and $\mathrm{y}$ stands for pile deflection (note that the soil resistance $\mathrm{p}$ is the product of pile deflection and the nonlinear spring constant). Instead of giving inputs for the nonlinear spring constant (i.e., the values of the spring constant as a function of pile deflection), p-y curves are given as inputs to the analysis in the $\mathrm{p}-\mathrm{y}$ method. Different $\mathrm{p}-\mathrm{y}$ curves have been developed over the years for different soil types, which give the magnitude of soil pressure as a function of the pile deflection (Reese et al. 1974, 1975, Reese and Welch 1975, Matlock 1970, Georgiadis 1983, O’Neill et al. 1990, Georgiadis et al. 1992, Yan and Bryne 1992, Gabr et al. 1994, Brown et al. 1994, Reese 1997, Wu et al. 1998, Bransby 1999, Zhang et al. 1999a, Ashour and Norris 2000). For the analysis, the pile is divided into small segments, and for each segment, a p-y curve is given as input. Depending on the magnitude of the deflection of a pile segment, the correct soil resistance is calculated from the p-y curve iteratively (since deflections and soil pressures are interdependent and since neither is known a priori, iterations are necessary to obtain their correct values) and solutions to the nonlinear fourth order differential equation are obtained using the finite difference method. With the development of the finite element method, analysis using beam finite elements have replaced the finite difference method in many calculations involving the subgrade-reaction approach or the p-y method (Stewart 2000, Hsiung and Chen 1997, Sogge 1981). Today, the p-y method is the most widely used method for calculating the response of laterally loaded piles.

The p-y method is often used for the analysis of pile groups as well. However, in order to use the standard $p-y$ curves developed for single piles, the $\mathrm{p}$ values are reduced to take into account the reduced resistance that a pile in a group offers due to pile interactions. The reduction in the values of $\mathrm{p}$ is generally done by multiplying $\mathrm{p}$ of the single-pile case by a multiplier $\mathrm{f}$, which depends, among other factors, on the number of piles in a group and their relative positions with respect to the pile in question (Salgado 2008). Different values and equations of the multiplier $\mathrm{f}$ have been proposed by various authors and are available in the literature (Brown et al. 1991, McVay et al. 1998, Mokwa 1999, Ilyas et al. 2004, Reese et al. 2006).

Using the p-y method or the subgrade-reaction approach, pile deflection is estimated as a function of applied load under working load conditions. In other words, design against the 
serviceability limit state of tolerable lateral deflection can be done using the p-y method. Since the serviceability limit state is the primary concern in the design of laterally loaded piles, the p-y method has gained huge popularity, particularly in the US. Over the years, several modifications and extensions of the beam-on-foundation approach and the p-y method have been made (Reddy and Valsangkar 1970, 1971, Madhav et al. 1971, Scott 1981, Aköz et al. 1981, Hsiung 2003, Shen and Teh 2004, Hsiung et al. 2006, Yang and Liang 2006). The characteristic load method of Duncan et al. (1994), in which dimensionless equations are developed from p-y analysis, and the strain wedge model of Ashour and Norris (2000), which considers a mobilized passive soil wedge in front of the pile to determine p-y curves, are examples of these methods.

The ultimate capacity due to the structural failure of a pile can be determined by using the p-y method if the plastic moment of the pile section is given as input to the p-y analysis. However, the p-y method cannot model the slip mechanism that would form if zones of soil adjacent to the pile were to yield. The beam-on-foundation approach can be used to calculate the ultimate capacity due to soil yielding, in which the soil is assumed to be perfectly plastic and limit soil resistance is used to estimate the ultimate lateral capacity. In such an approach, a limit soil pressure (i.e., passive pressure) is assumed to act throughout the length of the pile (in one direction above a certain center of rotation and in the opposite direction below it). The magnitude of the limit soil pressure is estimated, the positions of plastic hinge formation in the pile are located (required only for flexible piles), and force and moment equilibrium conditions are applied to calculate the ultimate (limit) load and moment that can act at the pile head (Broms 1964a, b, Poulos and Davis 1980, Fleming et al. 1992, Zhang et al. 2005).

\subsubsection{Continuum Approach}

Analysis of laterally loaded piles can be done by treating the soil surrounding the pile as a three-dimensional continuum. Such an approach is conceptually more appealing than the beamon-foundation approach because the interaction of the pile and the soil is indeed threedimensional in nature. Research in this direction was pioneered by Poulos (1971a), who treated the soil mass as an elastic continuum and the pile as a strip, which applied pressure on the continuum. He used Mindlin's solution (Mindlin 1936) for horizontal load acting at the interior of an elastic half space and applied a boundary integral technique to obtain pile deflection. 
However, the method is less popular than the p-y method, most likely because the analysis steps are relatively involved. The elastic analysis was extended to account for soil nonlinearity in an approximate way by assuming elastic-perfectly plastic soil (Poulos 1972, 1973, Davies and Budhu 1986, Budhu and Davies 1988). A similar boundary element analysis was performed by Banerjee and Davies (1978).

Today, the most versatile continuum-based method of analysis available is the finite element method. The method can take into account the three-dimensional interaction, and both elastic and nonlinear soils can be simulated by giving inputs of elastic constants (e.g., Young's modulus and Poisson's ratio) or by plugging in appropriate nonlinear constitutive relationships. Several researchers have used different forms of the finite element method (e.g., twodimensional analysis, three-dimensional analysis, finite elements coupled with Fourier techniques, finite elements coupled with finite difference, finite elements with substructuring) to analyze laterally loaded piles (Desai and Appel 1976, Randolph 1981, Kooijman and Vermeer 1988, Verruijt and Kooijman 1989, Trochanis et al. 1991, Bhowmik and Long 1991, Bransby 1999).

Other continuum-based analysis methods are also available (Baguelin et al. 1977, Pyke and Beikae 1984, Lee et al. 1987, Lee and Small 1991, Sun 1994a, Guo and Lee 2001, Einav 2005). However, these methods are rarely used by practitioners because either the analyses involve complicated mathematics and do not provide simple, practical steps for obtaining pile deflection or the methods are applicable only to linear elastic soils, which do not represent the reality of practical problems.

Continuum-based analyses have also been used to analyze pile groups. The boundary integral technique was used to capture the interaction between piles in groups (Poulos 1971b, Banerjee and Davies 1980, Basile 1999, Xu and Poulos 2000). The finite element method (Shibata et al. 1988, Chow 1987) and variational methods (Shen and Teh 2002) have been applied to pile-group problems as well. Because of the difficulties of applying the finite element method to large pile groups, Law and Lam (2001) proposed the application of periodic boundary conditions in finite element analysis of large pile groups. Additionally, some hybrid methods coupling both the continuum approach and the p-y have been used to model pile groups (Foch and Koch 1973, O’Neil et al. 1977, Horsnell et al. 1990). 


\subsection{Motivation for this Research Work}

The beam-on-foundation approach or the p-y method is an ideal candidate for laterally loaded-pile analysis from a practical point of view because of the ease with which solutions can be obtained. Solutions of the ordinary fourth order differential equation, even if nonlinear, can be obtained quickly. The assumption of an Euler-Bernoulli beam for the pile is satisfactory because most flexible piles are slender enough so that shear stresses and deformations within the piles can be neglected. However, springs are a poor representation of the surrounding soil. The interaction of a laterally loaded pile with the soil is three-dimensional in nature; the resistive properties of each element of soil surrounding the pile add up to generate the overall resistance against pile movement. Therefore, the nonlinear spring constant should be related to the resistive properties (e.g., stress-strain response) of the soil elements by taking into account the threedimensional interaction. Unfortunately, such a rigorous relationship is not available; for the beam-on-foundation approach, the spring constants are mostly estimated from empirical or semiempirical correlations (Francis 1964, Poulos and Davis 1980, Scott 1981, Bowles 1997, Hsiung and Chen 1997, Ashford and Juirnarongrit 2003).

The same limitation is applicable for the p-y curves as well. The method of preparation of the p-y curves developed from field observation and experience (Matlock 1970, Reese et al. 1974, 1975). The p-y curves used today are mostly obtained either by back fitting the results of numerical analysis (of the fourth-order beam-on-foundation equation) to match the observed deflections in the field or the results of model tests; or by correlating the curves with soil properties determined by laboratory or in-situ tests; or by comparing the results of p-y analysis with other numerical analyses (Matlock 1970, Reese et al. 1974, 1975, Brown and Kumar 1989, Yan and Byrne 1992, Brown et al. 1994, Gabr et al. 1994, Briaud 1997, Wu et al. 1998, Bransby 1999, Ashour and Norris 2000, Anderson et al. 2003). As a result, the p-y curves are sitespecific and do not take into account the three-dimensional pile-soil interaction. Considerable judgment is required for using the $\mathrm{p}$-y curves to predict proper pile response; in fact, analyses using the standard p-y curves often are reported to have failed to predict the actual pile loaddeflection response (Yan and Byrne 1992, Anderson et al. 2003, Kim et al. 2004). For example, Figure 1-11 (adapted from Kim et al. 2004) compares the p-y curves obtained from back calculation of the results of model tests on steel piles installed in Nak-Dong river sand, as reported by Kim et al. (2004), with the standard p-y curves for sands proposed by Reese et al. 
(1974), O’Neill and Murchinson (1983) and Wesselink et al. (1988) that are used in design. The figure clearly shows the deficiency of the standard p-y curves in producing reliable designs.

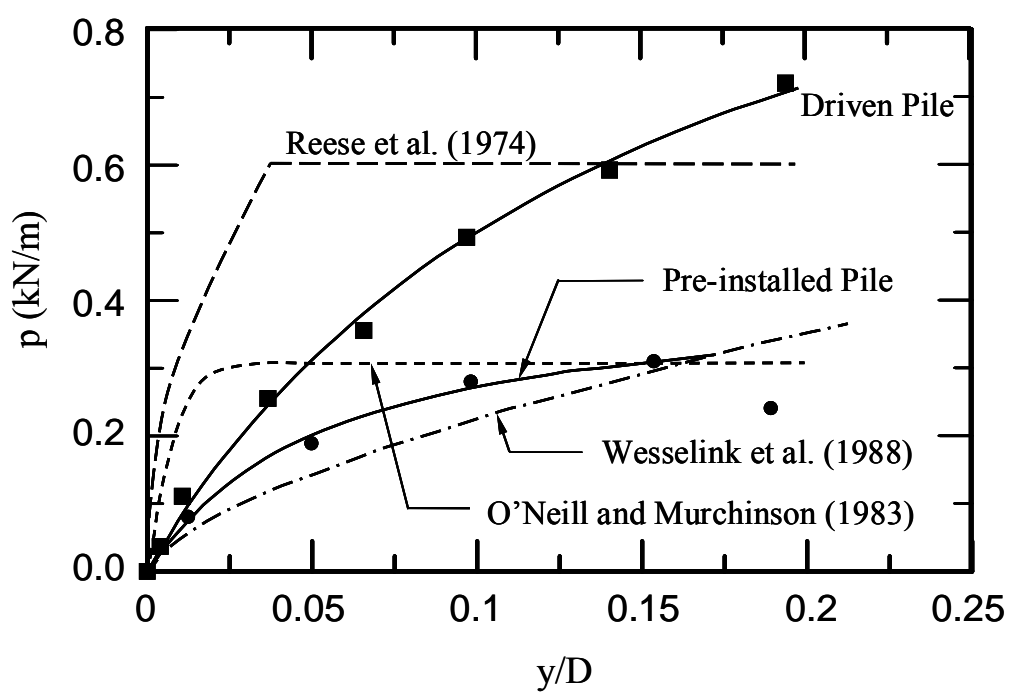

Figure 1-11 Comparison of Pile Resistance p versus Normalized Pile Deflection y/D (D is the Pile Diameter) Curves Obtained from Model Tests with the Standard Curves Available for Design (Adapted from Kim et al. 2004)

The finite element method, in its three-dimensional form, has the potential for producing realistic results for laterally loaded piles if appropriate soil constitutive relationships are used and if appropriate elements and domains are chosen for the soil and the pile. However, the enormous computation time and resources required for such an analysis prohibit practitioners from using finite elements in routine design.

An ideal method of analysis should have the rigor of a three-dimensional continuum approach, but should produce results as quickly as the beam-on-foundation approach. This is precisely the aim of this research. We hypothesize that a continuum-based, three-dimensional analysis can be developed for laterally loaded piles that rigorously relates the overall resistance of a soil mass to the soil constitutive relationship (i.e., stress-strain relationship). The analysis would take into account the actual pile-soil interaction and add up the resistances of each soil element to produce the total soil resistance. Consequently, the nonlinear properties of soil would be explicitly used to produce the nonlinear pile response, and the p-y curves would no longer be 
required. We further envisage connecting the continuum-based analysis to the to the beam-onfoundation approach so that the ordinary differential equation of the beam-on-foundation approach can be used to quickly obtain pile deflection. A particular aim is to develop the solutions in closed form so that expensive computer resources, essential for numerical analyses (e.g., by using finite elements), can be avoided.

\subsection{Scope of Present Study}

We develop a method of analysis of a laterally loaded pile embedded in a multi-layered soil medium and subjected to a horizontal force and moment at the pile head. Only static response is considered. We focus on serviceability and settlement-based limit states; i.e., we develop an analysis by which pile deflections can be predicted for the initial stages of loading (typically, a maximum pile-head deflection of the order of $25 \mathrm{~mm}$ is used as the criterion for serviceability limit state). The research starts with the development of a general framework, which shows logically how an improved beam-on-foundation model can be used to effectively analyze a laterally loaded pile embedded in a multi-layered soil. Then a continuum-based analysis is performed, which rigorously connects the properties of the three-dimensional continuum surrounding the pile to those of the soil springs, so that a one-to-one correspondence between the continuum-based approach and the beam-on-foundation approach can be established. The analysis is subsequently improved to incorporate the nonlinear stress-strain relationships of soils in the model. Finally, a method for pile group analysis of is presented.

In chapter 2, the pile is modeled as a beam resting on a multi-layered elastic foundation and solution for pile deflection is obtained analytically by using the method of initial parameters. In chapter 3, an elastic continuum model is introduced which is subsequently modified to incorporate soil nonlinearity in chapter 4. In chapter 5, we extend the analysis to pile groups. Finally, in chapter 6, we consolidate the research findings and propose future extensions of the research. 


\section{CHAPTER 2. LATERALLY LOADED PILE IN LAYERED ELASTIC MEDIUM: A BEAM- ON-ELASTIC-FOUNDATION APPROACH}

\subsection{Introduction}

In this chapter, we derive the governing differential equations for deflection of laterally loaded piles using a beam-on-elastic-foundation approach. Such an approach illustrates how simple idealizations of the statics and kinematics of pile-soil interaction can be used to model a laterally loaded pile as a beam resting on a foundation comprising of a series of springs. We derive the equations for multi-layered, elastic foundations. Then we obtain the analytical solutions for pile deflection, slope of the deflected curve (elastic curve), bending moment and shear force within each layer by using the method of initial parameters. Finally we discuss the modifications of the analytical solutions required for applying the solutions to long piles.

\subsection{Overview}

The beam-on-foundation model has been used in the past to analyze the response of laterally loaded piles (Broms 1964a, b, Matlock and Reese 1960, Fleming et al. 1992, Bowles 1997). Generally, a one-parameter foundation model represented by $k$ is considered ( $k$ being the spring constant per unit pile/beam length), although a two parameter model (which includes the shear parameter $t$ in addition to $k$ ) can also be used.

In order to account for soil nonlinearity, modification of the linear one-parameter model has been done by replacing the linear Winkler springs with nonlinear springs (McClelland and Focht 1958). For nonlinear springs, the spring constant $k$ (per unit pile or beam length) depends on the pile (beam) deflection $w$ (in general, the value of $k$ decreases with increasing $w$ ). Hence, the soil reaction per unit length $p=k w$ does not increase linearly with $w$. The nonlinear modification of the one-parameter model culminated in the development of the p-y method (Reese and Cox 1969, Matlock 1970, Reese et al. 1974, 1975, Reese and Van Impe 2001). In the p-y method, $k$ is no longer given as input (as a function of $w$ ); the nonlinear relationship of $k$ (or 
p) with $w$ are given as inputs to the analysis in the form of $p$-w curves, which are widely known in the literature as " $p-y$ " curves.

The one-parameter model assumes that the springs do not interact. This implies that the soil mass has only compressive resistance. Furthermore, the concentration of the load response at spring locations implies that there is no deflection beyond the loaded region (i.e., anywhere where there are no springs). In reality, both compression and shearing develop within the soil mass; consequently, deflections spread out beyond the loaded region (Figure 2-1). Thus, the one-parameter model cannot properly model the interaction between the pile and the soil. Different researchers have proposed different two-parameter models; these models result in the same differential equation but the interpretation of the second parameter $t$ is different in each of the models (Kerr 1964, Zhaohua and Cook 1983). Unfortunately, the two-parameter foundation model has rarely been used for laterally loaded pile analysis; on one occasion, Georgiadis and Butterfield (1982) assumed a two-parameter model to couple nonlinear soil shear force with the p-y method.

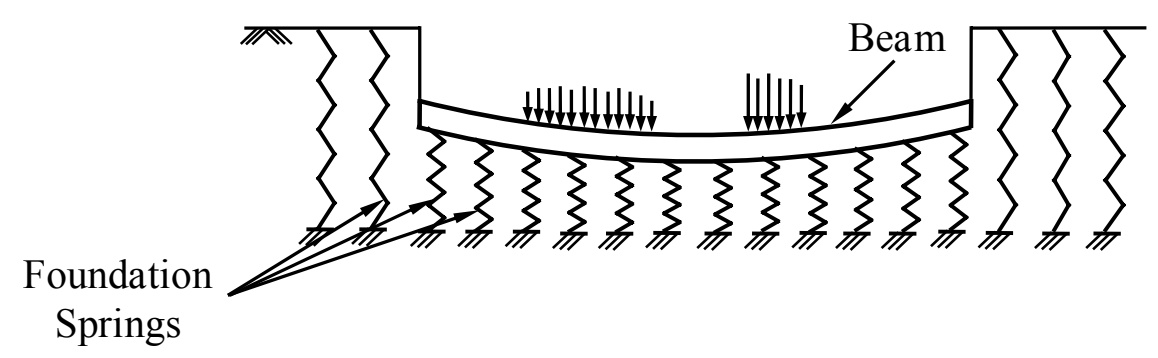

(a)

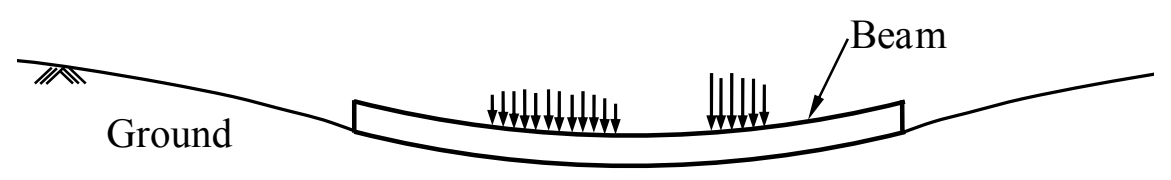

(b)

Figure 2-1 (a) Deflection Predicted by One-Parameter Model; (b) Actual Deflection Profile 
Another analysis approach is available in which the pile is treated as an Euler-Bernoulli beam and the surrounding soil mass is treated as an elastic continuum with a simplified assumption on the displacement field (Sun 1994a, Guo and Lee 2001). The analysis finally produces equations that are the same as the two-parameter-model equations. Thus, all these (one-parameter, two-parameter or continuum) approaches finally result in similar fourth-order differential equations, with pile deflection $w$ as the variable.

If the soil is assumed to be linear elastic, then the differential equations are also linear, and closed-form solutions for pile deflection can be obtained by solving the differential equations with proper boundary conditions. In the case of nonlinear soils, the equations are nonlinear, and numerical methods like the finite element method or the finite difference method are generally used to solve the problem. This applies equally to the continuum approach and to the p-y method, which is formulated using nonlinear (p-y) springs (McClelland and Focht 1958, Stewart 2000). For linear soils, general solutions of the fourth-order, linear differential equations are readily available (Hetényi 1946, Vlasov and Leont'ev 1966), and the four constants of integration can be determined from the pile boundary conditions (Sun 1994a, Guo and Lee 2001).

Soil layering is an important factor that affects laterally loaded pile response (Basu and Salgado 2007a). Layering has been taken into account approximately in some pile analyses by either assuming (typically) a linear variation of $k$ with depth or by proposing different $\mathrm{p}$-y curves for different soil depths (Broms 1965, Matlock and Reese 1960, Davisson 1970, Madhav et al. 1971, Valsangkar et al. 1973, Scott 1981, Ashour et al. 1998, Hsiung 2003). Such gradual variation of soil properties with depth has been assumed in many continuum-based analyses as well (Poulos 1973, Randolph 1981, Budhu and Davies 1988, Zhang et al. 2000, Banerjee and Davies 1978). However, in real field situations, discrete soil layers are often present and the assumption of linear (or similar) variation of soil properties does not properly represent the ground conditions. Analyses considering explicit layering (i.e., with multiple layers) are rather limited. Davisson and Gill (1963) analyzed a two-layer system using the p-y method. Georgiadis (1983) developed a method of developing p-y curves for layered soil profiles. A few continuum-based numerical analyses are also available (Pise 1982, Lee et al. 1987, Veruijt and Kooijman1989). Thus, in order to design laterally loaded piles for practical problems, a method of analysis by considering a multi-layered deposit needs to be developed. 
Although closed-form solutions of the fourth-order differential equation governing laterally loaded pile deflection exist for linear-elastic, homogeneous soils (Sun 1994a, Guo and Lee 2001), algebraic solutions for piles embedded in multi-layered soil deposits are difficult to obtain (albeit theoretically possible) because of the increased number of constants of integration. For example, for a four-layer laterally loaded pile problem, there are sixteen constants of integration (four constants per layer) that need to be determined algebraically by solving a set of sixteen simultaneous equations, arising due to the boundary conditions.

A finite element analysis using beam elements or a finite difference analysis can be used to analyze the problem (Scott 1981, Zhaohua and Cook, 1983, Sun 1994b). However, as described in chapter 3, our analysis requires the calculation of integrals, along depth, of the square of pile defection and slope. These integrations are performed numerically and require fine discretization of the pile along its length for accurate results. Therefore, if finite element or finite difference methods are used, the number of discretized pile elements will have to be very large resulting in increased computation time. Thus, obtaining analytical solutions of the piledeflection equation is necessary for our analysis.

We obtain analytical solutions by using the method of initial parameters (MIP), also known as the method of initial conditions (Hetényi 1946, Vlasov and Leont'ev 1966, Selvadurai 1979, Basu and Salgado 2007b), which yields the final analytical solutions without directly determining the integration constants. MIP was originally developed for solving problems of beams on elastic foundations (Hetényi 1946, Vlasov and Leont'ev 1966, Harr et al. 1969, Rao et al. 1971). The method is particularly useful when some form of discontinuity exists within the span of a beam. MIP has been applied to problems where the discontinuity is caused due to the application of concentrated forces at different points along the span of a beam (Vlasov and Leont'ev 1966, Harr et al. 1969, Rao et al. 1971).

In this chapter, we develop the equations for pile deflection following the beam-onelastic-foundation approach by considering both the one-parameter and two-parameter foundation models. This helps us to distinguish between the two models and to identify the advantages of the two-parameter model over the one-parameter model. We then modify the existing MIP to account for discontinuities along a pile caused by abrupt change in soil properties due to soil layering. This allows us to obtain analytical solutions for deflection of laterally loaded piles embedded in multi-layered elastic soils. We do not address the issue of soil 
nonlinearity in this chapter. However, the framework built in this chapter is subsequently improved in chapter 3 by incorporating a rigorous, continuum-based analysis, which culminates in the incorporation of soil nonlinearity in chapter 4.

\subsection{Problem Definition}

We consider a pile of constant flexural rigidity $E_{p} I_{p}\left(E_{p}\right.$ is the Young's modulus of the pile and $I_{p}$ is the second moment of inertia of the pile section) and length $L_{p}$ embedded in a multi-layered soil deposit (Figure 2-2). The soil is assumed to behave as a linear, elastic material. There are $n$ horizontal soil layers, with the bottom $\left(n^{\text {th }}\right)$ layer extending to infinity downward. The vertical depth to the bottom surface of any intermediate layer $i$ is $H_{i}$, which implies that the thickness of layer $i$ is $H_{i}-H_{i-1}$ with $H_{0}=0$. The pile top (head) is at the level of the ground surface. The bottom (base) of the pile is considered embedded in the $n^{\text {th }}$ layer. The pile is acted upon by a horizontal force $F_{a}$ and moment $M_{a}$ at the pile head.

We assume a right-handed Cartesian coordinate system $x-y-z$ with its origin at the center of the pile head such that the $z$ axis coincides with the pile axis and the $x-z$ plane coincides with the plane of the paper. The force $F_{a}$ acts in the $x$ direction and lies on the $x-z$ plane. The moment $M_{a}$, when expressed as a vector following the right-hand cork screw rule, acts into the plane of the paper (i.e. opposite $y$-direction). The bending of the pile takes place in the $x-z$ plane.

\subsection{Differential Equation and Boundary Conditions}

The pile is modeled as an Euler-Bernoulli beam. Considering the equilibrium of a pile cross section, as it bends under the action of the applied loads (Figure 2-3), we arrive at:

$$
\sigma_{n}=\frac{M x}{I_{p}}
$$

where $\sigma_{n}$ is the normal stress within the pile in the direction of the pile axis (i.e., $z$-axis); $x$ is the distance of the point (from the pile cross-section neutral axis) at which the normal stress $\sigma_{n}$ acts; $M=M(z)$ is the bending moment acting at the cross section (the positive sign convention for $M$ is shown in Figure 2-4). The corresponding normal strain (assuming compression positive) in the pile cross section can be obtained from equation (2-1) as: 

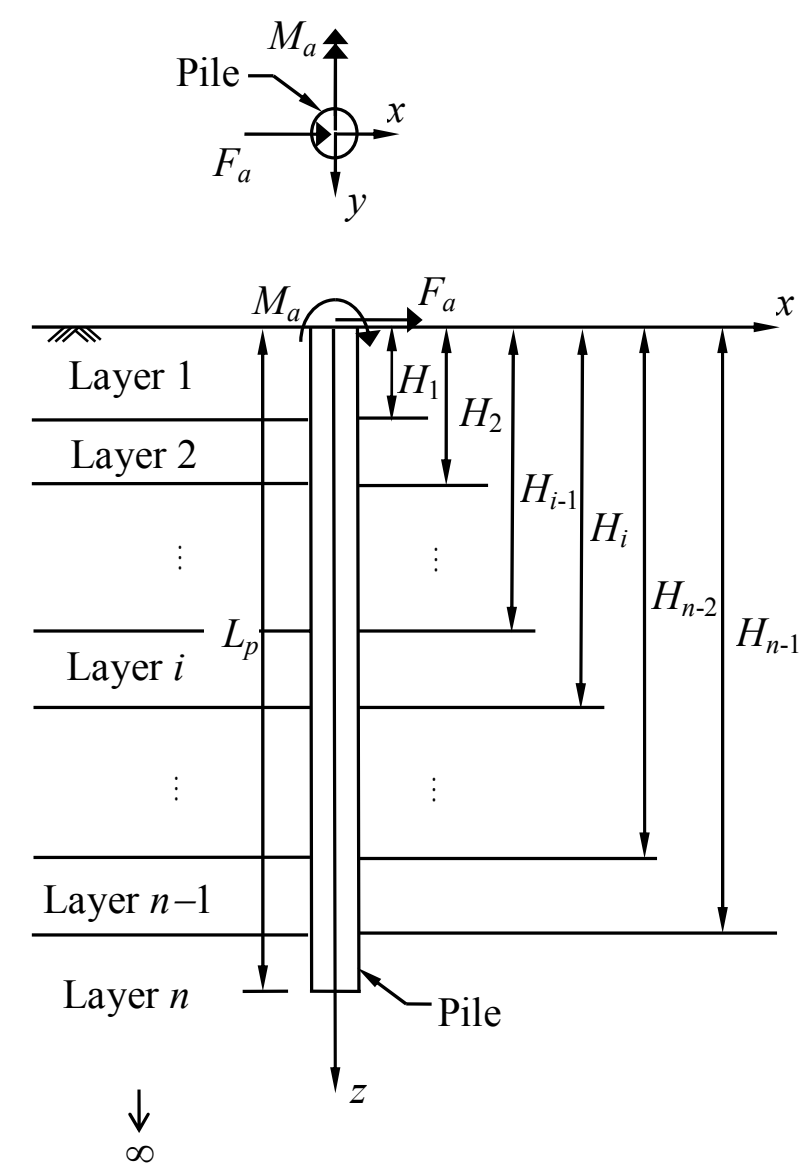

Figure 2-2 (a) A Laterally Loaded Pile in a Layered Soil Medium

$\varepsilon_{n}=\frac{M x}{E_{p} I_{p}}$

Considering the kinematics of the pile, we develop the following equation:

$\frac{d^{2} w_{i}}{d z^{2}}=\frac{\varepsilon_{n}}{x}$

where $w_{i}=w_{i}(z)$ is the lateral pile deflection at a depth $z$ (at a level corresponding to the $i^{\text {th }}$ layer) from the pile head.

Combining the statics and kinematics, we get (for the $i^{\text {th }}$ layer):

$E_{p} I_{p} \frac{d^{2} w_{i}}{d z^{2}}=M_{i}$

As we go down the pile by an infinitesimal distance $d z$, the shear force $S_{p}=S_{p}(z)$ on the pile cross section increases by $d S_{p}$ (the positive sign convention for $S_{p}$ is shown in Figure 2-4). 
Since the surrounding soil mass offers resistance to pile movement (Figure 2-3), the rate at which the shear force in the pile section increases over an infinitesimal length $d z$ can be related to the soil resistance $p=p(z)$ (produced by a "soil column" (Figure 2-3) of infinitesimal thickness $d z$ ) acting on the element (Figure 2-4).

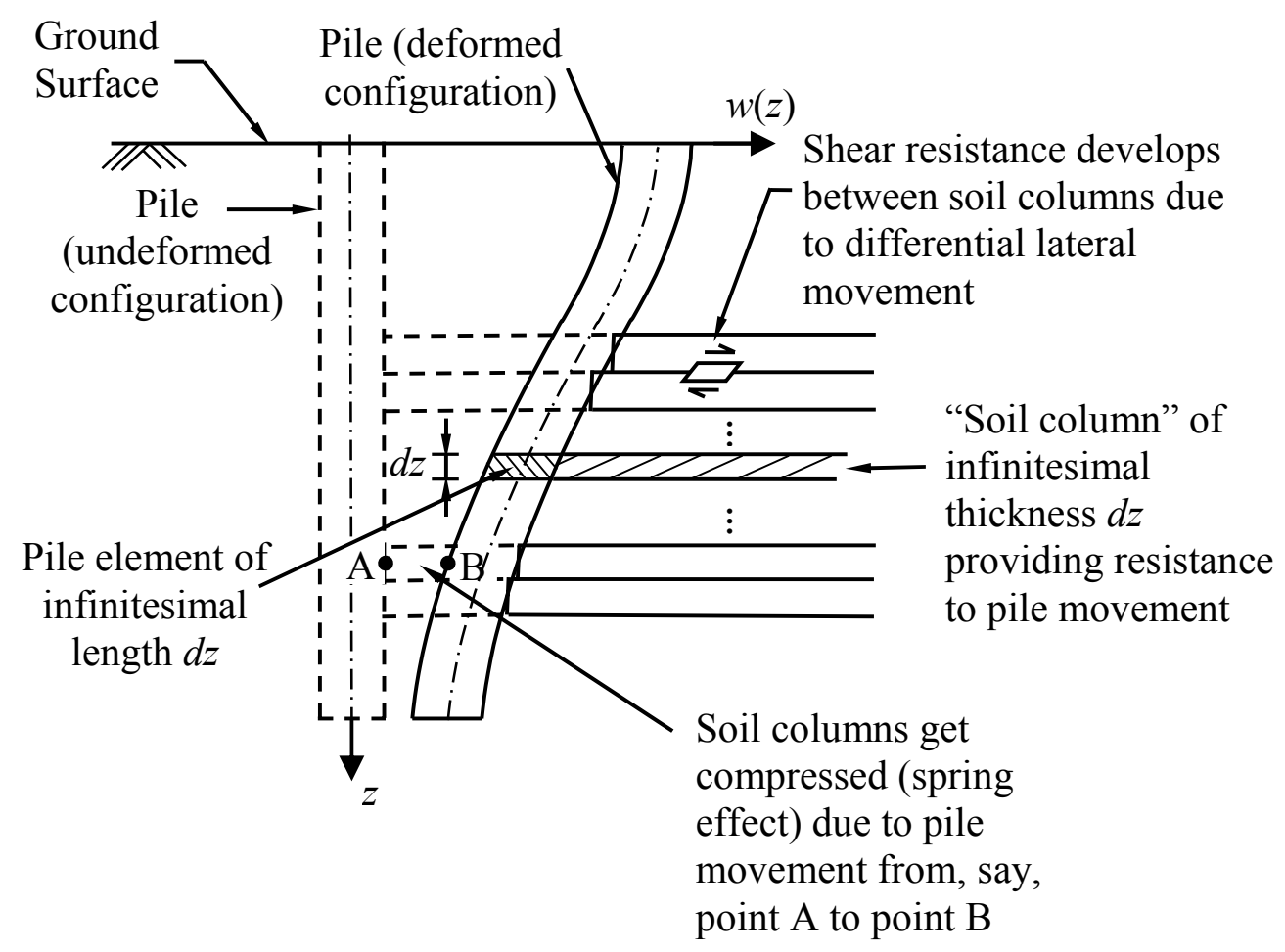

Figure 2-3 Pile-Soil Interaction

The soil resistance $p$ is a continuous, distributed force (per unit length) acting along the pile shaft in the negative $w(z)$ direction. The total soil resistance $p$ against pile movement has contributions from both the soil compressive resistance $p_{c}$ (since, the soil columns are compressed as the pile presses against them) and the soil shear resistance $p_{s}$ (since, the soil columns slide relative to each other due to differential change in pile deflection with depth) (Figure 2-3). Thus, for any layer $i$, we get (Figure 2-5):

$$
p_{i}=p_{c i}+p_{s i}
$$




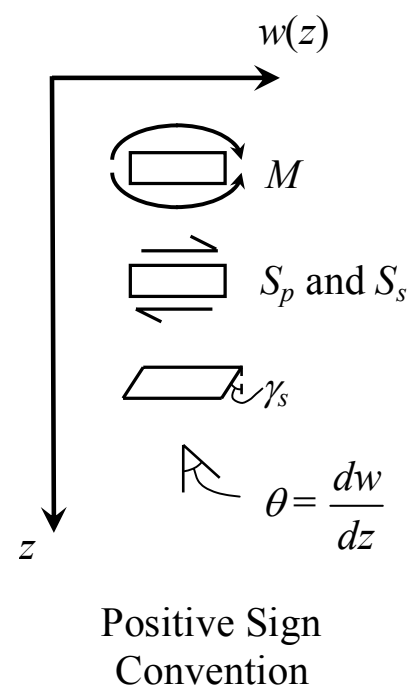

Figure 2-4 Sign Conventions Used

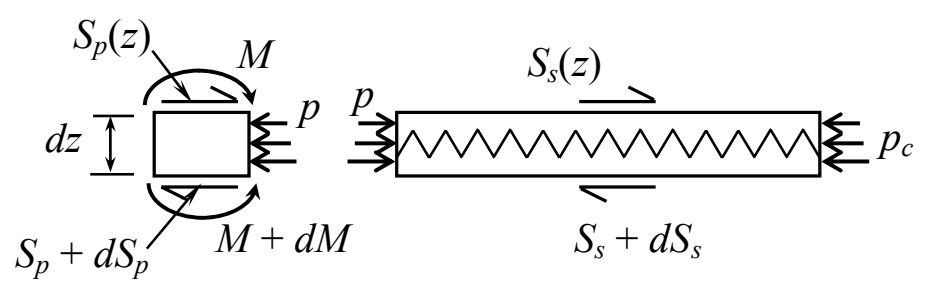

Pile element Soil column or spring

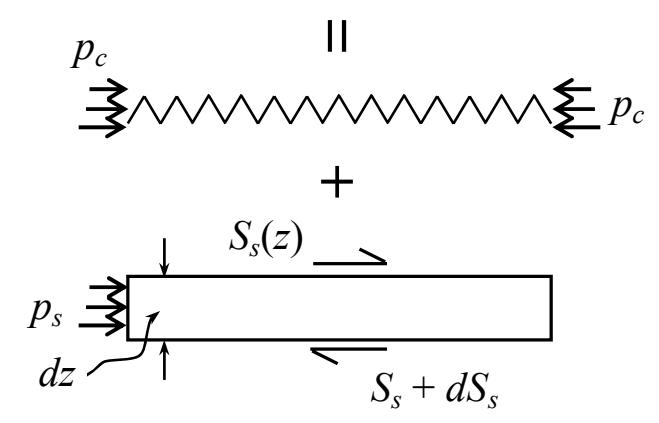

Figure 2-5 Equilibrium of Pile and Soil

The total soil resistance $p$ balances the change in pile shear force $d S_{p}$ over an infinitesimal length $d z$ and keeps the pile element in equilibrium (Figure 2-5). Therefore, considering the 
force equilibrium of the pile element for the $i^{\text {th }}$ layer, we get $S_{p i}-\left(S_{p i}+d S_{p i}\right)-p_{i} d z=0$, which gives:

$$
d S_{p i}=-p_{i} d z
$$

The increase $d M_{i}$ in the bending moment over the infinitesimal distance $d z$ can be related to the shear force $S_{p i}$ using moment equilibrium of the pile element (Figure 2-5) as $-M_{i}+\left(M+d M_{i}\right)-S_{p i} d z+p_{i} d z \frac{d z}{2}=0$. Neglecting the higher order term we get:

$d M_{i}=S_{p i} d z$

Equations (2-6) and (2-7) yield:

$$
\frac{d S_{p i}}{d z}=-p_{i}
$$

and

$$
\frac{d M_{i}}{d z}=S_{p i}
$$

Using equations (2-8) and (2-9), equation (2-4) can be rewritten as:

$$
E_{p} I_{p} \frac{d^{4} w_{i}}{d z^{4}}=-p_{i}
$$

Let us now consider the equilibrium of a soil column of infinitesimal thickness $d z$ at a depth $z$ as shown in Figure 2-5. As mentioned before, the soil resistance $p_{c}$ develops because of the compressive resistance of the soil column. Thus, in order to model the compressive resistance, the soil column can be replaced by an equivalent "soil spring" that reproduces the same compressive resistance. Consequently the part $p_{c}$ of the soil resistance in the $i^{\text {th }}$ layer can be expressed as:

$$
p_{c i}=k_{i} w_{i}
$$

where $k_{i}$ is the spring constant $\left(\mathrm{FL}^{-2}\right)$.

The soil columns move by different amounts as the pile deflects and bends in order to maintain displacement compatibility (Figure 2-3). Since soil offers resistance against shearing, shear forces are developed at the interfaces of adjacent soil columns due to their relative motion. The relative motion is not a constant with depth because the pile slope $\theta(=d w / d z)$ (i.e., the rate at which the pile deflection changes from one depth to another) is not a constant. Consequently, 
the soil shear force $S_{s}=S_{s}(z)$ is a function of $z$. As we move by an infinitesimal distance $d z$, the soil shear force increases from $S_{s}$ to $S_{s}+d S_{s}$ (the positive sign convention of $S_{s}$ is given in Figure 2-4). The change in the soil shear force $d S_{s}$ over a distance $d z$ is balanced by the soil resistance $p_{s}$ (Figure 2-5). Thus, considering the equilibrium of a soil element in the $i^{\text {th }}$ layer we get $p_{s i} d z+S_{s i}-\left(S_{s i}+d S_{s i}\right)=0$, which, along with equation (2-5) gives:

$$
d S_{s i}=p_{s i} d z=\left(p_{i}-p_{c i}\right) d z=\left(p_{i}-k_{i} w_{i}\right) d z
$$

The average soil shear stress arising from the soil shear force $S_{s i}$ can be related to the corresponding engineering shear strain $\gamma_{s}$ as:

$$
\gamma_{s}=\frac{1}{G_{s i}} \frac{S_{s i}}{A_{e}}=\frac{S_{s i}}{2 t_{i}}
$$

where $G_{s i}$ is the average soil shear modulus in the $i^{\text {th }}$ layer; $A_{e}$ is an equivalent area in the soil that relates the soil shear force to the corresponding average soil shear stress; and $t_{i}$ is the soil shear parameter, which is related to the soil shear modulus ( $t_{i}$ has a unit of force). The engineering shear strain $\gamma_{s}$ is also equal to the negative of the pile slope $\theta_{i}=\frac{d w_{i}}{d z}$ (a positive shear strain in the soil column causes a negative pile slope because of the sign convention for soil shear force shown in Figure 2-3). Therefore, from equation (2-13), we get:

$$
S_{s i}=-2 t_{i} \frac{d w_{i}}{d z}
$$

Using equations (2-12) and (2-14) we get:

$$
p_{i}=k_{i} w_{i}-2 t_{i} \frac{d^{2} w_{i}}{d z^{2}}=p_{c i}+p_{s i}
$$

The above equation also follows from the continuum model, as will be seen in chapter 3 , if some simplifying assumptions regarding the soil displacement fields are made.

In the case of the one-parameter model, the shear resistance of soil is neglected (i.e., $t_{i}=$ 0 , which means $p_{s i}=0$ ). Consequently, using equations (2-10) and (2-15) with $p_{s i}=0$, we get:

$$
E_{p} I_{p} \frac{d^{4} w_{i}}{d z^{4}}+k_{i} w_{i}=0
$$

In the case of the two-parameter model or the continuum model, in which the soil shearing resistance is taken into account, we get from equations (2-10) and (2-15): 


$$
E_{p} I_{p} \frac{d^{4} w_{i}}{d z^{4}}-2 t_{i} \frac{d^{2} w_{i}}{d z^{2}}+k_{i} w_{i}=0
$$

Equations (2-16a) and (2-17a) are the governing differential equations for pile deflection considering the one-parameter and the two-parameter (or the continuum) models, respectively.

The bending moment at any pile section at a depth $z$ is expressed in terms of pile deflection in equation (2-4). The shear force on any horizontal plane (which passes through both the pile and the soil) at any depth is the sum of the shear force $S_{p}$ acting in the pile section and the shear force $S_{s}$ acting in the soil. The shear force $S_{p}$ in the pile section can be expressed in terms of pile deflection using equations (2-4) and (2-7) as:

$$
S_{p i}=\frac{d M_{i}}{d z}=E_{p} I_{p} \frac{d^{3} w_{i}}{d z^{3}}
$$

The soil shear force $S_{s}$ is expressed in terms of pile deflection in equation (2-14). Hence, the total shear force $S$ at any depth $z$ within the $i^{\text {th }}$ layer can be expressed as:

$$
S_{i}=S_{p i}+S_{s i}=E_{p} I_{p} \frac{d^{3} w_{i}}{d z^{3}}-2 t_{i} \frac{d w_{i}}{d z}
$$

In the case of the one-parameter model, $S_{s i}$ in the above equation is equal to zero $\left(t_{i}=0\right)$; thus, the one-parameter model does not take into account the shear resistance of soil. The soil resistance $p_{i}$ is given by equation (2-11) for the one-parameter model and by equation (2-15) for the two-parameter (or continuum) model.

In an Euler-Bernoulli beam, the deflection, slope, bending moment and shear force is continuous along its span. In order to satisfy equilibrium at the beam ends (boundaries), any applied concentrated force (or reaction force) at the ends must be equal to the shear force at the corresponding sections (or the negative of the shear force, depending on the choice of sign convention). Similarly, any applied concentrated moment (or moment generated as a reaction due to restraints in rotation) at the ends must be equal to the bending moment at the corresponding sections (or the negative of the bending moment, depending on the choice of sign convention). In the case of laterally loaded piles, this is also true. These continuity and equilibrium requirements produce the boundary conditions for the governing differential equations (2-16a) and (2-17a).

For our problem, the boundary conditions for equations (2-16a) and (2-17a) at the pile head $(z=0)$ are: 


$$
\left.\left[E_{p} I_{p} \frac{d^{3} w_{1}}{d z^{3}}-2 t_{1} \frac{d w_{1}}{d z}\right]\right|_{z=0}=F_{a}
$$

and

$$
\left.\frac{d w_{1}}{d z}\right|_{z=0}=0
$$

or

$$
\left.E_{p} I_{p} \frac{d^{2} w_{1}}{d z^{2}}\right|_{z=0}=M_{a}
$$

At the interface between the $i^{\text {th }}$ and $(i+1)^{\text {th }}$ layer (i.e., at $\left.z=H_{i}\right)$, the boundary conditions are:

$$
\begin{aligned}
& \left.w_{i}\right|_{z=H_{i}}=\left.w_{i+1}\right|_{z=H_{i}} \\
& \left.\frac{d w_{i}}{d z}\right|_{z=H_{i}}=\left.\frac{d w_{i+1}}{d z}\right|_{z=H_{i}} \\
& \left.\frac{d^{2} w_{i}}{d z^{2}}\right|_{z=H_{i}}=\left.\frac{d^{2} w_{i+1}}{d z^{2}}\right|_{z=H_{i}} \\
& {\left.\left[E_{p} I_{p} \frac{d^{3} w_{i}}{d z^{3}}-2 t_{i} \frac{d w_{i}}{d z}\right]\right|_{z=H_{i}}=\left.\left[E_{p} I_{p} \frac{d^{3} w_{i+1}}{d z^{3}}-2 t_{i+1} \frac{d w_{i+1}}{d z}\right]\right|_{z=H_{i}}}
\end{aligned}
$$

At the pile base $\left(z=L_{p}\right)$ the boundary conditions are:

$$
\left.w_{n}\right|_{z=L_{p}}=0
$$

or

$$
\left.\left[E_{p} I_{p} \frac{d^{3} w_{n}}{d z^{3}}-2 t_{n} \frac{d w_{n}}{d z}\right]\right|_{z=L_{p}}=\left.\sqrt{2 k_{n} t_{n+1}} w_{n}\right|_{z=L_{p}}
$$

and

$$
\left.\frac{d w_{n}}{d z}\right|_{z=L_{p}}=0
$$

or

$$
\left.\frac{d^{2} w_{n}}{d z^{2}}\right|_{z=L_{p}}=0
$$


with $t_{i}=0$ for the one-parameter model.

Equation (2-20a) states that, at the pile head, the shear force is equal to the applied horizontal force. The direction of the applied force $F_{a}$ produces a positive shear force at the pile head. For two-parameter models, the shear force in the pile section and the shear force in the soil have to be considered together. Equations (2-21a) and (2-22a) state that at the pile head either the slope is equal to zero (this is the fixed-head condition, which might occur if a pile cap is present that may be considered to completely restrain pile head rotation) or the bending moment is equal to the applied moment (this is the free-head condition, which occurs when there is no pile cap present and the pile head is free to rotate). The direction of the applied moment $M_{a}$ produces a positive bending moment at the pile head. Equations (2-23a) through (2-26a) ensure the continuity of the deflection, slope, bending moment and shear force across adjacent layers. Equations (2-27a) and (2-28a) state that, at the pile base, either the deflection is equal to zero (this is the fixed-base condition, which may be assumed to occur when the pile is socketed into a very firm layer, like rock) or the shear force just above the base of the pile is equal to the shear force just below the base. It is worth mentioning here that, for the two-parameter or the continuum model, the shear force just below the pile base is equal to $\left.\sqrt{2 k_{n} t_{n+1}} w_{n}\right|_{z=L_{p}}$ (equation (2-28a)) as will be seen in chapter $3\left(t_{n+1}\right.$, defined in chapter 3 , quantifies the shear force at the pile base produced by the soil column that starts immediately below the pile and extends to infinity downward and has the same cross section as the pile). For the one-parameter model $\left(t_{i}=\right.$ 0 ), the shear force just below the pile base is equal to zero (which does not represent the real field condition and is a limitation of the one-parameter model). Equations (2-29a) and (2-30a) state that, at the pile base, either the slope is zero (valid for fixed-base condition) or the bending moment is zero (this is the free-base condition which might occur if the pile base floats in a soft layer of soil).

Equations (2-16a) and (2-17a) are normalized with respect to the length $L_{p}$ of the pile so that the results are applicable to any field conditions. We accomplished this by introducing the dimensionless variables $\tilde{z}=z / L_{p}$ and $\tilde{w}=w / L_{p}$ (note that $d z / d \tilde{z}=L_{p}$ ). The relationships of the successive differentiations of the normalized deflection function $\tilde{w}(\tilde{z})$ with respect to the normalized space (independent) variable $\tilde{z}$ with those of the original variables with dimensions are given by: 


$$
\begin{aligned}
& \frac{d \tilde{w}}{d \tilde{z}}=\frac{d}{d z}\left(\frac{w}{L_{p}}\right) \frac{d z}{d \tilde{z}}=\frac{1}{L_{p}} \frac{d w}{d z} L_{p}=\frac{d w}{d z} \\
& \frac{d^{2} \tilde{w}}{d \tilde{z}^{2}}=\frac{d}{d z}\left(\frac{d \tilde{w}}{d \tilde{z}}\right) \frac{d z}{d \tilde{z}}=\frac{d}{d z}\left(\frac{d w}{d z}\right) L_{p}=L_{p} \frac{d^{2} w}{d z^{2}} \\
& \frac{d^{3} \tilde{w}}{d \tilde{z}^{3}}=\frac{d}{d z}\left(\frac{d^{2} \tilde{w}}{d \tilde{z}^{2}}\right) \frac{d z}{d \tilde{z}}=\frac{d}{d z}\left(L_{p} \frac{d^{2} w}{d z^{2}}\right) L_{p}=L_{p}^{2} \frac{d^{3} w}{d z^{3}} \\
& \frac{d^{4} \tilde{w}}{d \tilde{z}^{4}}=\frac{d}{d z}\left(\frac{d^{3} \tilde{w}}{d \tilde{z}^{3}}\right) \frac{d z}{d \tilde{z}}=\frac{d}{d z}\left(L_{p}^{2} \frac{d^{3} w}{d z^{3}}\right) L_{p}=L_{p}^{3} \frac{d^{4} w}{d z^{4}}
\end{aligned}
$$

Using the above relationships in equations (2-16a) and (2-17a), we get the normalized form of the governing differential equations for the one-parameter and the two-parameter (or continuum) models as:

$$
\begin{aligned}
& \frac{d^{4} \tilde{w}_{i}}{d \tilde{z}^{4}}+\tilde{k}_{i} \tilde{w}_{i}=0 \\
& \frac{d^{4} \tilde{w}_{i}}{d \tilde{z}^{4}}-2 \tilde{t}_{i} \frac{d^{2} \tilde{w}_{i}}{d \tilde{z}^{2}}+\tilde{k}_{i} \tilde{w}_{i}=0
\end{aligned}
$$

where

$$
\begin{gathered}
\tilde{k}_{i}=\frac{k_{i} L_{p}^{4}}{E_{p} I_{p}} \\
\tilde{t}_{i}=\frac{t_{i} L_{p}^{2}}{E_{p} I_{p}}
\end{gathered}
$$

The slope $\theta_{i}=\frac{d w_{i}}{d z}$, bending moment $M_{i}$ (equation (2-4)), shear force $S_{i}$ (equation 2-19) and soil resistance $p_{i}$ (equation (2-15)) (in the $i^{\text {th }}$ layer) are respectively normalized and expressed in terms of the normalized pile deflection $\tilde{w}_{i}$ using the relationships given by equations (2-31a) through (2-31c) as:

$$
\begin{aligned}
& \widetilde{\theta}_{i}=\frac{d \widetilde{w}_{i}}{d \widetilde{z}} \\
& \widetilde{M}_{i}=\frac{M_{i} L_{p}}{E_{p} I_{p}}=\frac{d^{2} \widetilde{w}_{i}}{d \widetilde{z}^{2}}
\end{aligned}
$$




$$
\begin{aligned}
& \widetilde{S}_{i}=\frac{S_{i} L_{p}^{2}}{E_{p} I_{p}}=\frac{d^{3} \widetilde{w}_{i}}{d \widetilde{z}^{3}}-2 \widetilde{t}_{i} \frac{d \widetilde{w}_{i}}{d \widetilde{z}} \\
& \tilde{p}_{i}=\frac{p_{i} L_{p}^{3}}{E_{p} I_{p}}=\tilde{k}_{i} \tilde{w}_{i}-2 \tilde{t}_{i} \frac{d^{2} \tilde{w}_{i}}{d z^{2}}
\end{aligned}
$$

The applied force and moment can be similarly normalized as:

$$
\begin{gathered}
\widetilde{F}_{a}=\frac{F_{a} L_{p}^{2}}{E_{p} I_{p}} \\
\tilde{M}_{a}=\frac{M_{a} L_{p}}{E_{p} I_{p}}
\end{gathered}
$$

The normalized boundary conditions for equations (2-16b) and (2-17b) at the pile head $(\tilde{z}$ $=0)$ are:

$$
\left.\left[\frac{d^{3} \tilde{w}_{1}}{d \tilde{z}^{3}}-2 \tilde{t}_{1} \frac{d \tilde{w}_{1}}{d \tilde{z}}\right]\right|_{\tilde{z}=0}=\tilde{F}_{a}
$$

and

$$
\left.\frac{d \tilde{w}_{1}}{d \tilde{z}}\right|_{\tilde{z}=0}=0
$$

or

$$
\left.\frac{d^{2} \tilde{w}_{1}}{d \tilde{z}^{2}}\right|_{z=0}=\tilde{M}_{a}
$$

At the interface between the $i^{\text {th }}$ and $(i+1)^{\text {th }}$ layer (i.e., at $\tilde{z}=\tilde{H}_{i}$; where $\left.\tilde{H}_{i}=H_{i} / L_{p}\right)$, the boundary conditions are:

$$
\begin{aligned}
& \left.\tilde{w}_{i}\right|_{\tilde{z}=\tilde{H}_{i}}=\left.\tilde{w}_{i+1}\right|_{\tilde{z}=\tilde{H}_{i}} \\
& \left.\frac{d \tilde{w}_{i}}{d \tilde{z}}\right|_{\tilde{z}=\tilde{H}_{i}}=\left.\frac{d \tilde{w}_{i+1}}{d \tilde{z}}\right|_{\tilde{z}=\tilde{H}_{i}} \\
& \left.\frac{d^{2} \tilde{w}_{i}}{d \tilde{z}^{2}}\right|_{\tilde{z}=\tilde{H}_{i}}=\left.\frac{d^{2} \tilde{w}_{i+1}}{d \tilde{z}^{2}}\right|_{\tilde{z}=\tilde{H}_{i}} \\
& {\left.\left[\frac{d^{3} \tilde{w}_{i}}{d \tilde{z}^{3}}-2 \tilde{t}_{i} \frac{d \tilde{w}_{i}}{d \tilde{z}}\right]\right|_{\tilde{z}=\tilde{H}_{i}}=\left.\left[\frac{d^{3} \tilde{w}_{i+1}}{d \tilde{z}^{3}}-2 \tilde{t}_{i+1} \frac{d \tilde{w}_{i+1}}{d \tilde{z}}\right]\right|_{\tilde{z}=\tilde{H}_{i}}}
\end{aligned}
$$

At the pile base $(\tilde{z}=1)$ the boundary conditions are: 


$$
\left.\tilde{w}_{n}\right|_{\tilde{z}=1}=0
$$

or

$\left.\left[\frac{d^{3} \tilde{w}_{n}}{d \tilde{z}^{3}}-2 \tilde{t}_{n} \frac{d \tilde{w}_{n}}{d \tilde{z}}\right]\right|_{z=1}=\left.\sqrt{2 \tilde{k}_{n} \tilde{t}_{n+1}} \tilde{w}_{n}\right|_{z=1}$

and

$$
\left.\frac{d \tilde{w}_{n}}{d \tilde{z}}\right|_{z=1}=0
$$

or

$$
\left.\frac{d^{2} \tilde{w}_{n}}{d \tilde{z}^{2}}\right|_{\tilde{z}=1}=0
$$

with $\tilde{t}_{i}=0$ for the one-parameter model. Note that the normalized shear force at the pile base, for the two-parameter or the continuum model, is equal to $\left.\sqrt{2 \tilde{k}_{n} \tilde{t}_{n+1}} \tilde{w}_{n}\right|_{z=1}$ (equation (228b)) as we will show in chapter 3.

\subsection{General Solutions}

The general solution for both equations (2-16b) and (2-17b) is:

$$
\widetilde{w}_{i}(\widetilde{z})=C_{1}^{(i)} \Phi_{1}+C_{2}^{(i)} \Phi_{2}+C_{3}^{(i)} \Phi_{3}+C_{4}^{(i)} \Phi_{4}
$$

where, $C_{1}^{(i)}, C_{2}^{(i)}, C_{3}^{(i)}$ and $C_{4}^{(i)}$ are integration constants for the $i^{\text {th }}$ layer; and $\Phi_{1}, \Phi_{2}, \Phi_{3}$ and $\Phi_{4}$ are functions of $\tilde{z}$ that are individual solutions of the differential equation (2-16b) or (2-17b). The above general solution is not valid for long piles; we discuss the solution procedure for long piles later in the chapter.

Assuming a general solution of the form $\tilde{w}(\tilde{z})=e^{m \tilde{z}}$, the auxiliary equation corresponding to equation (2-16b) is given by:

$$
m^{4}+\tilde{k}_{i}=0
$$

Solution of equation (2-41) produces $m^{2}= \pm \sqrt{-\tilde{k}_{i}}$. Introducing the imaginary unit $i$ with the property $i^{2}=-1$, we can write $m^{2}= \pm i \sqrt{\tilde{k}_{i}}$, which produces $m= \pm \sqrt{ \pm i \sqrt{\tilde{k}_{i}}}$. Therefore, $m$ 
can be expressed as a complex number of the form $m=a+i b$, which yields $m^{2}=a^{2}-b^{2}+2 a b i= \pm i \sqrt{\tilde{k}_{i}}$. Since the real and imaginary part of a complex number are independent of each other, we get $a^{2}-b^{2}=0$ and $2 a b= \pm \sqrt{\tilde{k}_{i}}$. These two equations, when solved simultaneously, produce $a= \pm b= \pm \sqrt[4]{\tilde{k}_{i} / 4}$. Thus, we get $\tilde{w}(\tilde{z})=e^{m \tilde{z}}=e^{(a+i b) \tilde{z}}=e^{a \tilde{z}} e^{i b \tilde{z}}=e^{a \tilde{z}}(\cos b \tilde{z}+i \sin b \tilde{z})=e^{ \pm \sqrt{\frac{\tilde{k}_{i}}{4}}}\left\{\cos \left( \pm \sqrt{\tilde{k}_{i} / 4} \tilde{z}\right)+i \sin \left( \pm \sqrt{\tilde{k}_{i}} / 4 \tilde{z}\right)\right\}$. The four possible combinations of signs of $a$ and $b$ produce the functions $\Phi_{1}, \Phi_{2}, \Phi_{3}$ and $\Phi_{4}$ (which are individual solutions of equation (2-16b)) as combinations of trigonometric and exponential (or hyperbolic) functions as given in Table 2-1.

The auxiliary equation corresponding to equation $(2-17 b)$ is

$$
m^{4}-2 \tilde{t}_{i} m^{2}+\tilde{k}_{i}=0
$$

Solution of equation (2-42) produces $m= \pm \sqrt{\tilde{t}_{i} \pm \sqrt{\tilde{t}_{i}^{2}-\tilde{k}_{i}}}$, which leads to three conditions: 1) $\tilde{k_{i}}>\tilde{t}_{i}^{2}$, 2) ${\tilde{k_{i}}}_{i} \tilde{t}_{i}^{2}$ and 3) $\tilde{k}_{i}=\tilde{t}_{i}^{2}$. Since condition 3) can occur only under very special conditions (when the numerical value of the square of $\tilde{k_{i}}$ exactly equals the numerical value of $\tilde{t}_{i}$ ), we deal with the first two conditions only. Condition 1) makes $m$ a complex number of the form $m=a+i b$, similar to the case of equation (2-41), so that $m^{2}=a^{2}-b^{2}+2 a b i=\tilde{t}_{i} \pm i \sqrt{\tilde{k}_{i}-\tilde{t}_{i}^{2}}$, which yields $a^{2}-b^{2}=\tilde{t}_{i}$ and $2 a b=\sqrt{\tilde{k}_{i}-\tilde{t}_{i}^{2}}$. Solving these two equations simultaneously gives the expressions for $a$ and $b$, which are given in Table 2-1. Since $m$ is complex, $\Phi_{1}, \Phi_{2}, \Phi_{3}$ and $\Phi_{4}$ are again combinations of trigonometric and hyperbolic functions. To satisfy condition 2), $m$ does not require an imaginary part; consequently, $\Phi_{1}, \Phi_{2}$, $\Phi_{3}$ and $\Phi_{4}$ are exponential or hyperbolic functions. Table 2-1 gives the detailed expressions for the functions $\Phi_{1}, \Phi_{2}, \Phi_{3}$ and $\Phi_{4}$ as solutions to both equations (2-16b) and (2-17b).

The constants $C_{1}^{(i)}, C_{2}^{(i)}, C_{3}^{(i)}$ and $C_{4}^{(i)}$ need to be determined for different boundary conditions. However, for multi-layered soil deposits, obtaining algebraic expressions of these constants is extremely tedious. We avoid such algebra by using the method of initial parameters. 
Table 2-1 Functions in Equation (2-40) for Piles Crossing Multiple Soil Layers

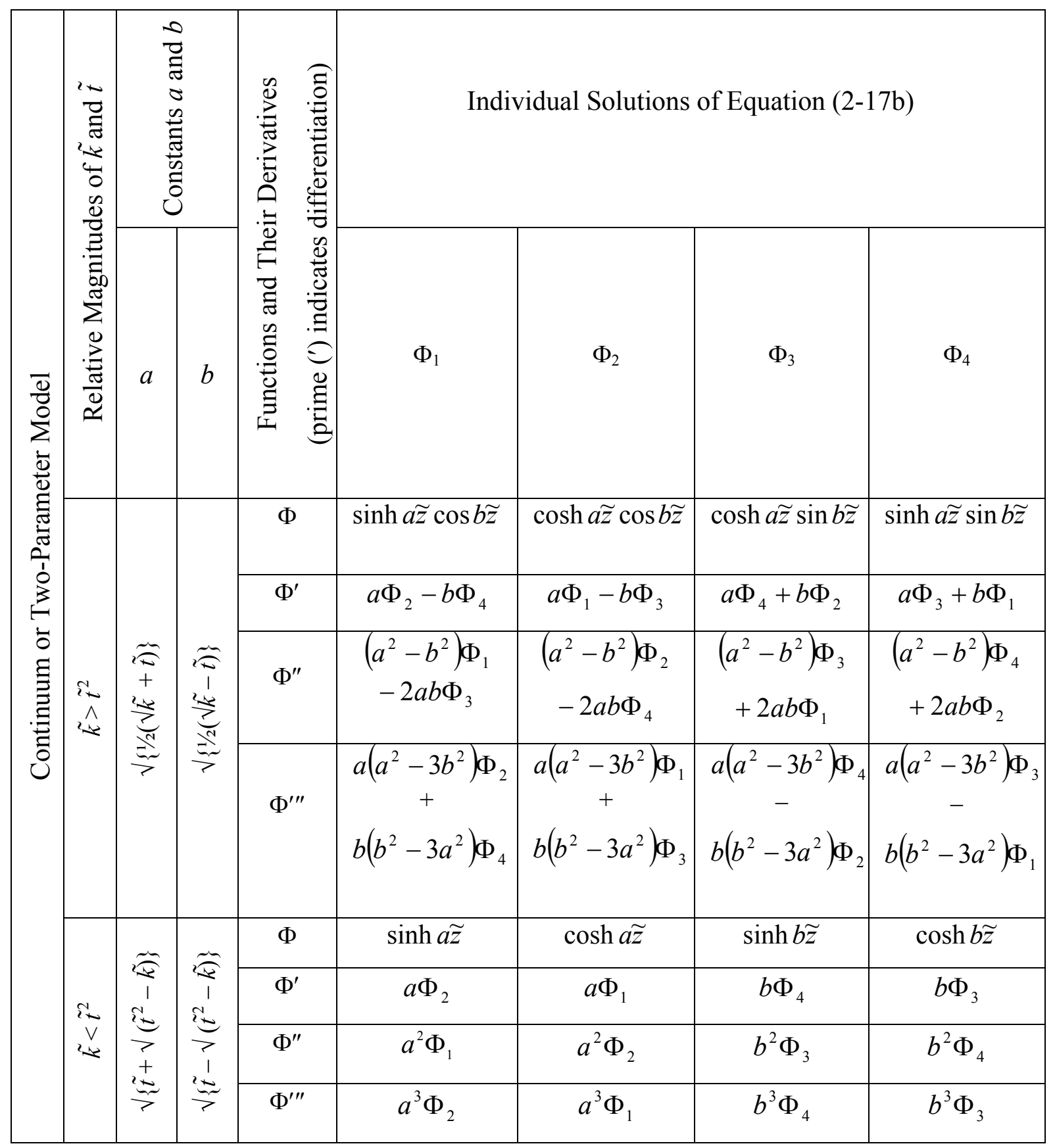

Note 1: For the individual solutions of equation (2-16b) (i.e., for the one-parameter model), the functions $\Phi_{1}, \Phi_{2}, \Phi_{3}$ and $\Phi_{4}$ are the same as for the case $\tilde{k}>\tilde{t}^{2}$ in Table 2-1, but $a=b=\sqrt[4]{\tilde{k} / 4}$.

Note 2: $\cosh a \tilde{z}=\frac{e^{a \tilde{z}}+e^{-a \tilde{z}}}{2}, \sinh a \tilde{z}=\frac{e^{a \tilde{z}}-e^{-a \tilde{z}}}{2}$. 


\subsection{Method of Initial Parameters}

We first outline the conceptual basis for MIP and then illustrate how it works for laterally loaded piles embedded in a layered soil. We illustrate the method for the two-parameter model (or the continuum model) for $\tilde{k_{i}}>\tilde{t}_{i}^{2}$. The method is also applicable for $\tilde{k_{i}} \leq{\tilde{t_{i}}}^{2}$ and for the oneparameter model, but it is sufficient to demonstrate the method for the case of $\tilde{k}_{i}>\tilde{t}_{i}^{2}$.

For the case of $\tilde{k_{i}}>\tilde{t}_{i}^{2}$, each of the functions $\Phi_{1}, \Phi_{2}, \Phi_{3}$ and $\Phi_{4}$ is a product of a trigonometric by a hyperbolic function, as shown in Table 2-1. Successive differentiations of these functions with respect to $\tilde{z}$ are also given in Table 2-1; these derivatives are required for obtaining the final solution for the laterally loaded-pile problem.

Let us first consider the top layer $(n=1)$. The following equations express the normalized deflection $\tilde{w}(\tilde{z})$, slope (equation (2-34)), bending moment (equation (2-35)) and shear force (equation (2-36)) for the top layer in terms of $\Phi_{1}, \Phi_{2}, \Phi_{3}$ and $\Phi_{4}$ as (see Table 2-1 under $\tilde{k}$ $>\tilde{t}^{2}$ for the differentiations of $\Phi_{1}, \Phi_{2}, \Phi_{3}$ and $\left.\Phi_{4}\right)$ :

$$
\begin{aligned}
& \widetilde{w}_{1}=C_{1} \Phi_{1}+C_{2} \Phi_{2}+C_{3} \Phi_{3}+C_{4} \Phi_{4} \\
& \tilde{\theta}_{1}=C_{1}\left(a \Phi_{2}-b \Phi_{4}\right)+C_{2}\left(a \Phi_{1}-b \Phi_{3}\right)+C_{3}\left(a \Phi_{4}+b \Phi_{2}\right)+C_{4}\left(a \Phi_{3}+b \Phi_{1}\right) \\
& \tilde{M}_{1}=C_{1}\left\{\left(a^{2}-b^{2}\right) \Phi_{1}-2 a b \Phi_{3}\right\}+C_{2}\left\{\left(a^{2}-b^{2}\right) \Phi_{2}-2 a b \Phi_{4}\right\} \\
& \quad+C_{3}\left\{\left(a^{2}-b^{2}\right) \Phi_{3}+2 a b \Phi_{1}\right\}+C_{4}\left\{\left(a^{2}-b^{2}\right) \Phi_{4}+2 a b \Phi_{2}\right\} \\
& \tilde{S}_{1}=C_{1}\left\{a\left(a^{2}-3 b^{2}\right) \Phi_{2}+b\left(b^{2}-3 a^{2}\right) \Phi_{4}\right\}+C_{2}\left\{a\left(a^{2}-3 b^{2}\right) \Phi_{1}+b\left(b^{2}-3 a^{2}\right) \Phi_{3}\right\} \\
& +C_{3}\left\{a\left(a^{2}-3 b^{2}\right) \Phi_{4}-b\left(b^{2}-3 a^{2}\right) \Phi_{2}\right\}+C_{4}\left\{a\left(a^{2}-3 b^{2}\right) \Phi_{3}-b\left(b^{2}-3 a^{2}\right) \Phi_{1}\right\} \\
& \quad-2 \tilde{t}_{1}\left\{C_{1}\left(a \Phi_{2}-b \Phi_{4}\right)+C_{2}\left(a \Phi_{1}-b \Phi_{3}\right)+C_{3}\left(a \Phi_{4}+b \Phi_{2}\right)+C_{4}\left(a \Phi_{3}+b \Phi_{1}\right)\right\}
\end{aligned}
$$

where the constants $C_{1}, C_{2}, C_{3}$ and $C_{4}$ and the functions $\Phi_{1}, \Phi_{2}, \Phi_{3}$ and $\Phi_{4}$ are valid for layer 1 $\left(0 \leq \tilde{z} \leq H_{1}\right)$. Let the normalized pile deflection, slope, bending moment and shear force at the pile head (i.e., at $\tilde{z}=0$ ) be $\tilde{w}_{0}^{(1)}, \tilde{\theta}_{0}^{(1)}, \tilde{M}_{0}^{(1)}$ and $\tilde{S}_{0}^{(1)}$, respectively. If we substitute $\tilde{z}=0$ in equations (2-43) through (2-46) (note that, for $\tilde{z}=0, \Phi_{1}=\Phi_{3}=\Phi_{4}=0$ and $\Phi_{2}=1$ ), then we can express $\tilde{w}_{0}^{(1)}, \tilde{\theta}_{0}^{(1)}, \tilde{M}_{0}^{(1)}$ and $\tilde{S}_{0}^{(1)}$ as:

$$
\begin{aligned}
& \tilde{w}_{0}^{(1)}=C_{2} \\
& \tilde{\theta}_{0}^{(1)}=\left.\frac{d \tilde{w}_{1}}{d \tilde{z}}\right|_{\tilde{z}=0}=C_{1} a+C_{3} b
\end{aligned}
$$




$$
\begin{aligned}
& \tilde{M}_{0}^{(1)}=\left.\frac{d^{2} \tilde{w}_{1}}{d \tilde{z}^{2}}\right|_{z=0}=C_{1}\left(a^{2}-b^{2}\right)+C_{4}(2 a b) \\
& \tilde{S}_{0}^{(1)}=\left.\left[\frac{d^{3} \tilde{w}_{1}}{d \tilde{z}^{3}}-2 \tilde{t}_{1} \frac{d \tilde{w}_{1}}{d \tilde{z}}\right]\right|_{z=0}=C_{1} a\left(a^{2}-3 b^{2}\right)-C_{3} b\left(b^{2}-3 a^{2}\right)-2 \tilde{t}_{1}\left(C_{1} a+C_{3} b\right)
\end{aligned}
$$

Solving equations (2-47) through (2-50) simultaneously for $C_{1}, C_{2}, C_{3}$ and $C_{4}$ yields:

$$
\begin{aligned}
& C_{1}=\frac{\left\{\left(3 a^{2}-b^{2}\right)-2 \tilde{t}_{1}\right\} \tilde{\theta}_{0}^{(1)}-\tilde{S}_{0}^{(1)}}{2 a\left(a^{2}+b^{2}\right)} \\
& C_{2}=\tilde{w}_{0}^{(1)} \\
& C_{3}=-\frac{\left\{\left(a^{2}-3 b^{2}\right)-2 \tilde{t}_{1}\right\} \tilde{\theta}_{0}^{(1)}-\tilde{S}_{0}^{(1)}}{2 b\left(a^{2}+b^{2}\right)} \\
& C_{4}=-\frac{\left(a^{2}-b^{2}\right) \tilde{w}_{0}^{(1)}-\tilde{M}_{0}^{(1)}}{2 a b}
\end{aligned}
$$

If we now substitute $C_{1}, C_{2}, C_{3}$ and $C_{4}$ in equations (2-43) through (2-46), we get the expression for pile displacement, slope, bending moment and shear force in the top layer as:

$$
\begin{aligned}
\tilde{w}_{1}(z)= & {\left[\Phi_{2}-\frac{\left(a^{2}-b^{2}\right) \Phi_{4}}{2 a b}\right] \tilde{w}_{0}^{(1)} } \\
& +\left[-\frac{\left(a^{3} \Phi_{3}-3 a^{2} b \Phi_{1}-3 a b^{2} \Phi_{3}+b^{3} \Phi_{1}\right)-2 \tilde{t}_{1}\left(a \Phi_{3}-b \Phi_{3}\right)}{2 a b\left(a^{2}+b^{2}\right)}\right] \tilde{\theta}_{0}^{(1)} \\
& +\frac{\Phi_{4}}{2 a b} \tilde{M}_{0}^{(1)}+\left[\frac{a \Phi_{3}-b \Phi_{1}}{2 a b\left(a^{2}+b^{2}\right)}\right] \tilde{S}_{0}^{(1)} \\
\tilde{\theta}_{1}(z)= & {\left[a \Phi_{1}-b \Phi_{3}-\frac{\left(a^{2}-b^{2}\right)\left(a \Phi_{3}+b \Phi_{1}\right)}{2 a b}\right] \tilde{w}_{0}^{(1)} } \\
+ & {\left[-\frac{a^{2} \Phi_{4}-2 a b \Phi_{2}-b^{2} \Phi_{4}-2 \tilde{t}_{1} \Phi_{4}}{2 a b}\right] \tilde{\theta}_{0}^{(1)}+\frac{a \Phi_{3}+b \Phi_{1}}{2 a b} \tilde{M}_{0}^{(1)}+\frac{\Phi_{4}}{2 a b} \tilde{S}_{0}^{(1)} }
\end{aligned}
$$




$$
\begin{aligned}
\tilde{M}_{1}(z)= & {\left[\frac{\Phi_{4}\left(a^{4}+b^{4}+2 a^{2} b^{2}\right)}{2 a b}\right] \tilde{w}_{0}^{(1)} } \\
& +\left[-\frac{a^{3} \Phi_{3}-a^{2} b \Phi_{1}+a b^{2} \Phi_{3}-b^{3} \Phi_{1}-2 \tilde{t}_{1}\left(a \Phi_{3}+b \Phi_{1}\right)}{2 a b}\right] \tilde{\theta}_{0}^{(1)} \\
& +\frac{\left(a^{2}-b^{2}\right) \Phi_{4}+2 a b \Phi_{2}}{2 a b} \tilde{M}_{0}^{(1)}+\frac{a \Phi_{3}+b \Phi_{1}}{2 a b} \tilde{S}_{0}^{(1)} \\
\tilde{S}_{1}(z)= & {\left[-\frac{1}{2 a b}\left\{a^{5} \Phi_{3}+a^{4} b \Phi_{1}+2 a^{3} b^{2} \Phi_{3}+2 a^{2} b^{3} \Phi_{1}+a b^{4} \Phi_{3}+b^{5} \Phi_{1}\right.\right.} \\
& \left.\left.-2 \tilde{t}_{1}\left(a^{3} \Phi_{3}-a^{2} b \Phi_{1}+a b^{2} \Phi_{3}-b^{3} \Phi_{1}\right)\right\}\right] \tilde{w}_{0}^{(1)} \\
& +\left[\left\{-\frac{a^{4}+2 a^{2} b^{2}+b^{4}-4 \tilde{t}_{1}\left(a^{2}-b^{2}\right)}{2 a b}-2 \tilde{t}_{1}^{2}\right\} \Phi_{4}\right] \tilde{\theta}_{0}^{(1)} \\
& +\left[\frac{a^{3} \Phi_{3}+3 a^{2} b \Phi_{1}-3 a b^{2} \Phi_{3}-b^{3} \Phi_{1}-2 \tilde{t}_{1}\left(a \Phi_{3}+b \Phi_{1}\right)}{2 a b}\right] \tilde{M}_{0}^{(1)} \\
& +\left[\frac{a^{2} \Phi_{4}+2 a b \Phi_{2}-b^{2} \Phi_{4}-2 \tilde{t}_{1} \Phi_{4}}{2 a b}\right] \tilde{S}_{0}^{(1)}
\end{aligned}
$$

Thus, the normalized pile deflection, slope, bending moment and shear force can be expressed in terms of $\tilde{w}_{0}^{(1)}, \tilde{\theta}_{0}^{(1)}, \tilde{M}_{0}^{(1)}$ and $\tilde{S}_{0}^{(1)}$ along with some known functions of $\Phi_{1}, \Phi_{2}, \Phi_{3}$ and $\Phi_{4}$. If $\tilde{w}_{0}^{(1)}, \tilde{\theta}_{0}^{(1)}, \tilde{M}_{0}^{(1)}$ and $\tilde{S}_{0}^{(1)}$ are known, then we can determine the deflection, slope, bending moment and shear force at any point within the first layer. This is the basic idea behind the method of initial parameters. The quantities $\tilde{w}_{0}^{(1)}, \tilde{\theta}_{0}^{(1)}, \tilde{M}_{0}^{(1)}$ and $\tilde{S}_{0}^{(1)}$ are called the initial parameters for the first layer; the corresponding section of the pile ( $\tilde{z}=0$ in this case) is called the initial section. The expressions in square brackets, containing $\Phi_{1}, \Phi_{2}, \Phi_{3}$ and $\Phi_{4}$, associated with the initial parameters, are called the influence functions or influence coefficients.

Any section within the first layer can be chosen as the initial section and equations similar to equations (2-55) through (2-58) can be obtained. The expressions for the influence coefficients will change if a different initial section is chosen. 
Extending the method to account for multi-layered soil, we can state that the normalized deflection $\widetilde{w}_{i}$, the slope $\tilde{\theta}_{i}$, the bending moment $\tilde{M}_{i}$ and the shear force $\tilde{S}_{i}$ within any layer $i$ can be expressed as:

$$
\begin{aligned}
& \widetilde{w}_{i}(\widetilde{z})=\widetilde{w}_{0}^{(i)} K_{w w}^{(i)}+\widetilde{\theta}_{0}^{(i)} K_{w \theta}^{(i)}+\widetilde{M}_{0}^{(i)} K_{w M}^{(i)}+\widetilde{S}_{0}^{(i)} K_{w S}^{(i)} \\
& \widetilde{\theta}_{i}(\widetilde{z})=\widetilde{w}_{0}^{(i)} K_{\theta w}^{(i)}+\widetilde{\theta}_{0}^{(i)} K_{\theta \theta}^{(i)}+\widetilde{M}_{0}^{(i)} K_{\theta M}^{(i)}+\widetilde{S}_{0}^{(i)} K_{\theta S}^{(i)} \\
& \widetilde{M}_{i}(\widetilde{z})=\widetilde{w}_{0}^{(i)} K_{M w}^{(i)}+\widetilde{\theta}_{0}^{(i)} K_{M \theta}^{(i)}+\widetilde{M}_{0}^{(i)} K_{M M}^{(i)}+\widetilde{S}_{0}^{(i)} K_{M S}^{(i)} \\
& \widetilde{S}_{i}(\widetilde{z})=\widetilde{w}_{0}^{(i)} K_{S w}^{(i)}+\widetilde{\theta}_{0}^{(i)} K_{S \theta}^{(i)}+\widetilde{M}_{0}^{(i)} K_{S M}^{(i)}+\widetilde{S}_{0}^{(i)} K_{S S}^{(i)}
\end{aligned}
$$

where $\widetilde{w}_{0}^{(i)}, \widetilde{\theta}_{0}^{(i)}, \widetilde{M}_{0}^{(i)}$ and $\widetilde{S}_{0}^{(i)}$ are the initial parameters of the $i^{\text {th }}$ layer. Each layer requires its own initial section. The influence coefficients are denoted by $K$ 's. If the initial parameters and the influence coefficients are known for a layer, then the deflection, slope, bending moment, and shear force can be obtained as a function of depth.

We now illustrate how MIP can be used for layered soil with the help of an example. We consider, for our example, a pile embedded in a three-layer soil medium with ${\tilde{k_{i}}}{\tilde{t_{i}}}_{i}^{2}$ for all the layers (Figure 2-6). There is no restraint at the pile head (i.e., it is free to translate and rotate). The same is true for the pile base.

The first step is to choose an appropriate initial section for each layer. We choose the pile head and base as the initial sections for the top and the bottom (third) layers respectively. For the middle (second) layer we choose the upper interface as the initial section. Thus, the initial section for the top layer (layer 1) is at $\tilde{z}=0$. The four initial parameters are $\tilde{w}_{0}^{(1)}, \tilde{\theta}_{0}^{(1)}$, $\tilde{M}_{0}^{(1)}$ and $\tilde{S}_{0}^{(1)}$, which are the normalized deflection, slope, bending moment and shear force at $\tilde{z}=$ 0 . The initial section for the bottom layer (layer 3 ) is at $\tilde{z}=1$. The corresponding initial parameters are $\tilde{w}_{0}^{(3)}, \tilde{\theta}_{0}^{(3)}, \tilde{M}_{0}^{(3)}$ and $\tilde{S}_{0}^{(3)}$. The initial section for the middle layer (layer 2) is at $\tilde{z}$ $=\tilde{H}_{1}$ with the initial parameters $\tilde{w}_{0}^{(2)}, \tilde{\theta}_{0}^{(2)}, \tilde{M}_{0}^{(2)}$ and $\tilde{S}_{0}^{(2)}$.

Next we impose the boundary conditions for the pile head and base on the initial parameters for the two end layers. For a free pile head, equations (2-20b) and (2-22b) are valid; this yields:

$$
\begin{aligned}
& \widetilde{S}_{0}^{(1)}=\widetilde{F}_{a} \\
& \widetilde{M}_{0}^{(1)}=\widetilde{M}_{a}
\end{aligned}
$$


For a free pile base, equations (2-28b) and (2-30b) are valid, which yields:

$$
\begin{aligned}
& \tilde{S}_{0}^{(3)}=\sqrt{2 \tilde{k}_{3} \tilde{t}_{4}} \tilde{w}_{0}^{(3)} \\
& \tilde{M}_{0}^{(3)}=0
\end{aligned}
$$

Thus, the initial parameters $\tilde{S}_{0}^{(1)}, \tilde{M}_{0}^{(1)}, \tilde{S}_{0}^{(3)}$ and $\tilde{M}_{0}^{(3)}$ are now known. The equations for the normalized deflection, slope, bending moment and shear force for layers 1 and 3 can now be rewritten by substituting the known initial parameters into equation (2-59) through (2-62) (for $i=$ 1 and 3) as:

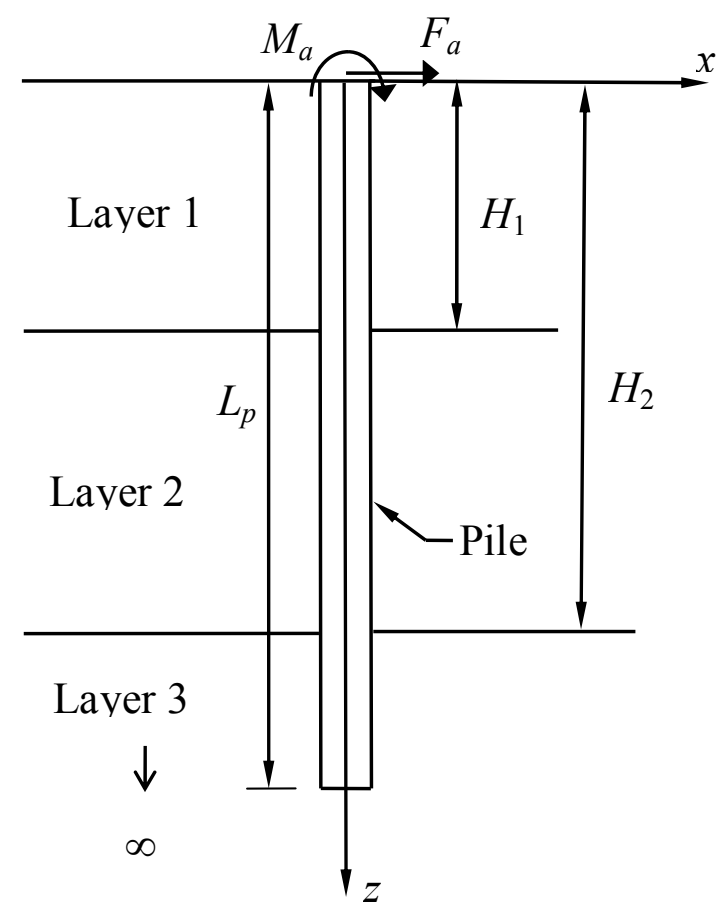

Figure 2-6 A Laterally Loaded Pile in a Three-Layer Medium

$$
\begin{aligned}
& \widetilde{w}_{1}=\widetilde{w}_{0}^{(1)} K_{w w}^{(1)}+\widetilde{\theta}_{0}^{(1)} K_{w \theta}^{(1)}+\widetilde{M}_{a} K_{w M}^{(1)}+\widetilde{F}_{a} K_{w S}^{(1)} \\
& \widetilde{\theta}_{1}=\widetilde{w}_{0}^{(1)} K_{\theta w}^{(1)}+\widetilde{\theta}_{0}^{(1)} K_{\theta \theta}^{(1)}+\widetilde{M}_{a} K_{\theta M}^{(1)}+\widetilde{F}_{a} K_{\theta S}^{(1)} \\
& \widetilde{M}_{1}=\widetilde{w}_{0}^{(1)} K_{M w}^{(1)}+\widetilde{\theta}_{0}^{(1)} K_{M \theta}^{(1)}+\widetilde{M}_{a} K_{M M}^{(1)}+\widetilde{F}_{a} K_{M S}^{(1)} \\
& \widetilde{S}_{1}=\widetilde{w}_{0}^{(1)} K_{S w}^{(1)}+\widetilde{\theta}_{0}^{(1)} K_{S \theta}^{(1)}+\widetilde{M}_{a} K_{S M}^{(1)}+\widetilde{F}_{a} K_{S S}^{(1)}
\end{aligned}
$$




$$
\begin{aligned}
& \tilde{w}_{3}=\tilde{w}_{0}^{(3)} K_{w w}^{(3)}+\tilde{\theta}_{0}^{(3)} K_{w \theta}^{(3)}+\sqrt{2 \tilde{k}_{3} \tilde{t}_{4}} \tilde{w}_{0}^{(3)} K_{w S}^{(3)}=\tilde{w}_{0}^{(3)} K_{w w}^{(3)}+\tilde{\theta}_{0}^{(3)} K_{w \theta}^{(3)} \\
& \tilde{\theta}_{3}=\tilde{w}_{0}^{(3)} K_{\theta w}^{(3)}+\tilde{\theta}_{0}^{(3)} K_{\theta \theta}^{(3)}+\sqrt{2 \tilde{k}_{3} \tilde{t}_{4}} \tilde{w}_{0}^{(3)} K_{\theta S}^{(3)}=\tilde{w}_{0}^{(3)} K_{\theta w}^{(3)}+\tilde{\theta}_{0}^{(3)} K_{\theta \theta}^{(3)} \\
& \tilde{M}_{3}=\tilde{w}_{0}^{(3)} K_{M w}^{(3)}+\tilde{\theta}_{0}^{(3)} K_{M \theta}^{(3)}+\sqrt{2 \tilde{k}_{3} \tilde{t}_{4}} \tilde{w}_{0}^{(3)} K_{M S}^{(3)}=\tilde{w}_{0}^{(3)} K_{M w}^{\prime(3)}+\tilde{\theta}_{0}^{(3)} K_{M \theta}^{(3)} \\
& \tilde{S}_{3}=\tilde{w}_{0}^{(3)} K_{S w}^{(3)}+\tilde{\theta}_{0}^{(3)} K_{S \theta}^{(3)}+\sqrt{2 \tilde{k}_{3} \tilde{t}_{4}} \tilde{w}_{0}^{(3)} K_{S S}^{(3)}=\tilde{w}_{0}^{(3)} K_{S w}^{(3)}+\tilde{\theta}_{0}^{(3)} K_{S \theta}^{(3)}
\end{aligned}
$$

where $K_{* w}^{\prime(3)}=K_{*_{w}}^{(3)}+\sqrt{2 \tilde{t}_{3} \tilde{k}_{4}} K_{* S}^{(3)}$. Equations (2-67) through (2-70) are valid for layer 1 while equations (2-71) through (2-74) are valid for layer 3.

Now we impose the interface boundary conditions on the initial parameters. This leads to a set of algebraic equations consisting of the unknown influence coefficients and initial parameters. In our example problem, there are two interfaces $\left(\tilde{z}=\tilde{H}_{1}\right.$ and $\left.\tilde{z}=\tilde{H}_{2}\right)$, and the boundary conditions at these interfaces are given by equations (2-23b) through (2-26b) with $i=1$ and 2 for $\tilde{z}=\tilde{H}_{1}$ and $\tilde{z}=\tilde{H}_{2}$, respectively. There are four equations per interface, resulting in eight simultaneous equations, which can be written in matrix form as:

$$
\begin{aligned}
& {\left[\begin{array}{cccccccc}
K_{w w}^{(1)} & K_{w \theta}^{(1)} & -1 & 0 & 0 & 0 & 0 & 0 \\
K_{\theta w}^{(1)} & K_{\theta \theta}^{(1)} & 0 & -1 & 0 & 0 & 0 & 0 \\
K_{M w}^{(1)} & K_{M \theta}^{(1)} & 0 & 0 & -1 & 0 & 0 & 0 \\
K_{S w}^{(1)} & K_{S \theta}^{(1)} & 0 & 0 & 0 & -1 & 0 & 0 \\
0 & 0 & K_{w w}^{(2)} & K_{w \theta}^{(2)} & K_{w M}^{(2)} & K_{w S}^{(2)} & -1 & 0 \\
0 & 0 & K_{\theta w}^{(2)} & K_{\theta \theta}^{(2)} & K_{\theta M}^{(2)} & K_{\theta S}^{(2)} & 0 & -1 \\
0 & 0 & K_{M w}^{(2)} & K_{M \theta}^{(2)} & K_{M M}^{(2)} & K_{M S}^{(2)} & -K_{M w}^{(3)} & -K_{M \theta}^{(3)} \\
0 & 0 & K_{S w}^{(2)} & K_{S \theta}^{(2)} & K_{S M}^{(2)} & K_{S S}^{(2)} & -K_{S w}^{(3)} & -K_{S \theta}^{(3)}
\end{array}\right]\left[\begin{array}{c}
\tilde{w}_{0}^{(1)} \\
\tilde{\theta}_{0}^{(1)} \\
\tilde{w}_{0}^{(2)} \\
\tilde{\theta}_{0}^{(2)} \\
\tilde{M}_{0}^{(2)} \\
\tilde{S}_{0}^{(2)} \\
\tilde{w}_{0}^{(3)} \\
\tilde{\theta}_{0}^{(3)}
\end{array}\right]} \\
& =\left[\begin{array}{c}
-\tilde{M}_{a} K_{w M}^{(1)}-\tilde{F}_{a} K_{w S}^{(1)} \\
-\tilde{M}_{a} K_{\theta M}^{(1)}-\tilde{F}_{a} K_{\theta S}^{(1)} \\
-\tilde{M}_{a} K_{M M}^{(1)}-\tilde{F}_{a} K_{M S}^{(1)} \\
-\tilde{M}_{a} K_{S M}^{(1)}-\tilde{F}_{a} K_{S S}^{(1)} \\
0 \\
0 \\
0 \\
0
\end{array}\right]
\end{aligned}
$$

We refer to equation (2-75) as the "matrix equation". In the matrix equation, the first four rows are valid at $\tilde{z}=\tilde{H}_{1}$ while the remaining four are valid at $\tilde{z}=\tilde{H}_{2}$. If, in equation (2-75), we assume that the influence coefficients $(K$ 's) are known, then all the unknown initial 
parameters for the different layers can be obtained. Thus, the matrix in the left-hand side of equation (2-75) has to be inverted to obtain the initial parameters. Such inversions were done using the LU decomposition method (Chapra and Canale 1998).

We can determine the influence coefficients for use in equation (2-75) by referring back to equations (2-55) through (2-58). In these equations $\tilde{M}_{0}^{(1)}$ and $\tilde{S}_{0}^{(1)}$ are now replaced by $\tilde{M}_{a}$ and $\tilde{F}_{a}$, respectively, because of the boundary conditions of the example problem given by equations (2-63) and (2-64). Hence, by comparing the coefficients of $\tilde{w}_{0}^{(1)}, \tilde{\theta}_{0}^{(1)}, \tilde{M}_{a}$ and $\tilde{F}_{a}$ in equations (2-55) through (2-58) with those in equations (2-67) through (2-70), we obtain the influence coefficients for layer 1 as:

$$
\begin{aligned}
& K_{w w}^{(1)}=\Phi_{2}-\frac{\left(a^{2}-b^{2}\right) \Phi_{4}}{2 a b} \\
& K_{w \theta}^{(1)}=-\frac{a^{3} \Phi_{3}-3 a^{2} b \Phi_{1}-3 a b^{2} \Phi_{3}+b^{3} \Phi_{1}-2 \tilde{t}_{1}\left(a \Phi_{3}-b \Phi_{3}\right)}{2 a b\left(a^{2}+b^{2}\right)} \\
& K_{w M}^{(1)}=\frac{\Phi_{4}}{2 a b} \\
& K_{w S}^{(1)}=\frac{a \Phi_{3}-b \Phi_{1}}{2 a b\left(a^{2}+b^{2}\right)} \\
& K_{\theta w}^{(1)}=\frac{a \Phi_{1}-b \Phi_{3}-\frac{\left(a^{2}-b^{2}\right)\left(a \Phi_{3}+b \Phi_{1}\right)}{2 a b}}{2 a b} \\
& K_{\theta \theta}^{(1)}=-\frac{a^{2} \Phi_{4}-2 a b \Phi_{2}-b^{2} \Phi_{4}-2 \tilde{t}_{1} \Phi_{4}}{2 a b} \\
& K_{M \theta}^{(1)}=-\frac{a^{3} \Phi_{3}-a^{2} b \Phi_{1}+a b^{2} \Phi_{3}-b^{3} \Phi_{1}-2 \tilde{t}_{1}\left(a \Phi_{3}+b \Phi_{1}\right)}{2 a b}=\frac{a \Phi_{3}+b \Phi_{1}}{2 a b} \\
& K_{M w}^{(1)}=\frac{\Phi_{4}\left(a^{4}+b^{4}+2 a^{2} b^{2}\right)}{2 a b} \\
& K_{\theta S} \\
& K_{\theta}
\end{aligned}
$$




$$
\begin{aligned}
K_{M M}^{(1)}= & \frac{\left(a^{2}-b^{2}\right) \Phi_{4}+2 a b \Phi_{2}}{2 a b} \\
K_{M S}^{(1)}= & \frac{a \Phi_{3}+b \Phi_{1}}{2 a b} \\
K_{S w}^{(1)}= & -\frac{1}{2 a b}\left\{a^{5} \Phi_{3}+a^{4} b \Phi_{1}+2 a^{3} b^{2} \Phi_{3}+2 a^{2} b^{3} \Phi_{1}+a b^{4} \Phi_{3}+b^{5} \Phi_{1}\right. \\
& \left.-2 \tilde{t}_{1}\left(a^{3} \Phi_{3}-a^{2} b \Phi_{1}+a b^{2} \Phi_{3}-b^{3} \Phi_{1}\right)\right\} \\
K_{S \theta}^{(1)}= & \left\{-\frac{a^{4}+2 a^{2} b^{2}+b^{4}-4 \tilde{t}_{1}\left(a^{2}-b^{2}\right)}{2 a b}-2 \tilde{t}_{1}^{2}\right\} \Phi_{4} \\
K_{S M}^{(1)}= & \frac{a^{3} \Phi_{3}+3 a^{2} b \Phi_{1}-3 a b^{2} \Phi_{3}-b^{3} \Phi_{1}-2 \tilde{t}_{1}\left(a \Phi_{3}+b \Phi_{1}\right)}{2 a b} \\
K_{S S}^{(1)}= & \frac{a^{2} \Phi_{4}+2 a b \Phi_{2}-b^{2} \Phi_{4}-2 \tilde{t}_{1} \Phi_{4}}{2 a b}
\end{aligned}
$$

For the second (middle) layer, the following equations need to be solved simultaneously in terms of $C_{1}, C_{2}, C_{3}$ and $C_{4}$ (note that these constants are different from the constants of layer 1):

$$
\begin{aligned}
\widetilde{w}_{0}^{(2)}= & C_{1} \phi_{1}+C_{2} \phi_{2}+C_{3} \phi_{3}+C_{4} \phi_{4} \\
\widetilde{\theta}_{0}^{(2)}= & C_{1}\left(a \phi_{2}-b \phi_{4}\right)+C_{2}\left(a \phi_{1}-b \phi_{3}\right)+C_{3}\left(a \phi_{4}+b \phi_{2}\right)+C_{4}\left(a \phi_{3}+b \phi_{1}\right) \\
\widetilde{M}_{0}^{(2)}= & C_{1}\left\{\left(a^{2}-b^{2}\right) \phi_{1}-2 a b \phi_{3}\right\}+C_{2}\left\{\left(a^{2}-b^{2}\right) \phi_{2}-2 a b \phi_{4}\right\} \\
& +C_{3}\left\{\left(a^{2}-b^{2}\right) \phi_{3}+2 a b \phi_{1}\right\}+C_{4}\left\{\left(a^{2}-b^{2}\right) \phi_{4}+2 a b \phi_{2}\right\} \\
\widetilde{S}_{0}^{(2)}= & C_{1}\left\{a\left(a^{2}-3 b^{2}\right) \phi_{2}+b\left(b^{2}-3 a^{2}\right) \phi_{4}\right\}+C_{2}\left\{a\left(a^{2}-3 b^{2}\right) \phi_{1}+b\left(b^{2}-3 a^{2}\right) \phi_{3}\right\} \\
+ & C_{3}\left\{a\left(a^{2}-3 b^{2}\right) \phi_{4}-b\left(b^{2}-3 a^{2}\right) \phi_{2}\right\}+C_{4}\left\{a\left(a^{2}-3 b^{2}\right) \phi_{3}-b\left(b^{2}-3 a^{2}\right) \phi_{1}\right\} \\
& -2 \tilde{t}_{2}\left\{C_{1}\left(a \phi_{2}-b \phi_{4}\right)+C_{2}\left(a \phi_{1}-b \phi_{3}\right)+C_{3}\left(a \phi_{4}+b \phi_{2}\right)+C_{4}\left(a \phi_{3}+b \phi_{1}\right)\right\}
\end{aligned}
$$

where $\phi_{1}, \phi_{2}, \phi_{3}$ and $\phi_{4}$ are the values of $\Phi_{1}, \Phi_{2}, \Phi_{3}$ and $\Phi_{4}$, respectively, at the initial section of the second layer (i.e., at $\tilde{z}=\tilde{H}_{1}$ ). After obtaining the expressions of $C_{1}, C_{2}, C_{3}$ and $C_{4}$, the same procedure as for layer 1 needs to be followed to obtain the influence coefficients of layer 2 . Similarly, the influence coefficients of layer 3 can be obtained by solving the following equations for $C_{1}, C_{2}, C_{3}$ and $C_{4}$ :

$$
\widetilde{w}_{0}^{(3)}=C_{1} \phi_{1}+C_{2} \phi_{2}+C_{3} \phi_{3}+C_{4} \phi_{4}
$$




$$
\begin{aligned}
\widetilde{\theta}_{0}^{(3)} & =C_{1}\left(a \phi_{2}-b \phi_{4}\right)+C_{2}\left(a \phi_{1}-b \phi_{3}\right)+C_{3}\left(a \phi_{4}+b \phi_{2}\right)+C_{4}\left(a \phi_{3}+b \phi_{1}\right) \\
\tilde{M}_{0}^{(3)} & =0=C_{1}\left\{\left(a^{2}-b^{2}\right) \phi_{1}-2 a b \phi_{3}\right\}+C_{2}\left\{\left(a^{2}-b^{2}\right) \phi_{2}-2 a b \phi_{4}\right\} \\
& +C_{3}\left\{\left(a^{2}-b^{2}\right) \phi_{3}+2 a b \phi_{1}\right\}+C_{4}\left\{\left(a^{2}-b^{2}\right) \phi_{4}+2 a b \phi_{2}\right\} \\
\tilde{S}_{0}^{(3)} & =\sqrt{2 \tilde{k}_{3} \tilde{t}_{4}} \tilde{w}_{0}^{(3)}=C_{1}\left\{a\left(a^{2}-3 b^{2}\right) \phi_{2}+b\left(b^{2}-3 a^{2}\right) \phi_{4}\right\} \\
& +C_{2}\left\{a\left(a^{2}-3 b^{2}\right) \phi_{1}+b\left(b^{2}-3 a^{2}\right) \phi_{3}\right\}+C_{3}\left\{a\left(a^{2}-3 b^{2}\right) \phi_{4}-b\left(b^{2}-3 a^{2}\right) \phi_{2}\right\} \\
& +C_{4}\left\{a\left(a^{2}-3 b^{2}\right) \phi_{3}-b\left(b^{2}-3 a^{2}\right) \phi_{1}\right\}-2 \tilde{t}_{3}\left\{C_{1}\left(a \phi_{2}-b \phi_{4}\right)+C_{2}\left(a \phi_{1}-b \phi_{3}\right)\right. \\
& \left.+C_{3}\left(a \phi_{4}+b \phi_{2}\right)+C_{4}\left(a \phi_{3}+b \phi_{1}\right)\right\}
\end{aligned}
$$

where $\phi_{1}, \phi_{2}, \phi_{3}$ and $\phi_{4}$ are the values of $\Phi_{1}, \Phi_{2}, \Phi_{3}$ and $\Phi_{4}$, respectively at $\tilde{z}=1$.

Once the influence coefficients are known, the unknown initial parameters are determined by solving equation (2-75). The initial parameters and influence coefficients are then used to find the normalized pile deflection, slope, bending moment and shear force at any pile section by using equations (2-67) through (2-70) for layer 1, (2-59) through (2-62) for layer 2 (with $i=2$ ), and (2-71) through (2-74) for layer 3 .

Evidently, MIP can be used for solving problems with any number of layers and for different boundary conditions. It is important to note that the matrix equations for the different cases (arising due to soil layering and pile boundary conditions) are different. However, for a given pile head boundary condition, the influence coefficients for the top layer are the same irrespective of the number of soil layers and of the boundary conditions at the pile base. Similarly, for the bottom layer, the influence coefficients are independent of the number of soil layers and of the pile head boundary conditions. Likewise, for any intermediate layer, the expressions of the influence coefficients are the same irrespective of the pile head and base boundary conditions and the number of layers present. This makes the determination of the influence coefficient expressions and subsequent programming easy because the expressions for the top and bottom layers do not vary from problem to problem if the boundary conditions remain the same, and the expressions for intermediate layers are always the same, being also independent of the head and base boundary conditions. 
The influence coefficients and matrix equations for one-, two-, three- and four-layer soil deposits have been obtained for all possible boundary conditions (i.e., free or fixed pile head with free or fixed pile base). The expressions are given in Basu (2006).

\subsection{Solution for Long Piles}

\subsubsection{General Solution}

Long piles are piles whose response is identical to that of infinitely long piles. For such piles, the boundary conditions at the pile base do not affect the pile response. Pile response depends on the relative magnitudes of the pile and soil stiffness and on the pile geometry (pile slenderness ratio). Depending on such ratios, as illustrated in Figure 2-7, a pile of length $5 \mathrm{~m}$ may behave as a long pile, while a 10-m-long pile may not.

Figure 2-7a shows the deflection versus depth profiles of a set of 500 -mm-diameter concrete piles $\left(E_{p}=2.4 \times 10^{6} \mathrm{kPa}\right)$ of lengths $2.5 \mathrm{~m}, 5 \mathrm{~m}$ and $6 \mathrm{~m}$, respectively, embedded in a dense sand layer $(k=130 \mathrm{MPa}$, and $t=7.9 \mathrm{MN})$. A horizontal load of $100 \mathrm{kN}$ is applied to all the piles at the pile head. The piles are assumed to be free at the head; however, both free and fixed conditions for the base are considered. It is evident from Figure 2-7a that the deflection profile of the $5 \mathrm{~m}$-long pile is independent of the pile base conditions and that the pile behaves as a long pile. This happens because the ground is stiff enough when compared to the pile so that it prevents lateral movement of the pile beyond a certain depth $h_{f}(=3.3 \mathrm{~m}$ in this example), known as the depth of fixity, which is less than the length of the pile. The 6-m-long pile also behaves as a long pile because the depth of fixity remains at $3.3 \mathrm{~m}$, which is less than the pile length (Figure 2-7a). However, the response of the 2.5-m-long pile depends on the base condition because the pile has a length less than the depth of fixity; consequently, it does not behave as a long pile.

Figure 2-7b shows the deflection versus depth profile of a 1-m-diameter concrete pile of length $10 \mathrm{~m}$, embedded in a soft clay deposit with $k=11.6 \mathrm{MPa}$ and $t=6.8 \mathrm{MN}$ and subjected to a $100 \mathrm{kN}$ horizontal load at the head. The pile head is assumed to be free, while both free and fixed conditions are considered for the base. The deflection profile clearly shows the influence of the pile base condition, which is a contrast to the response of the 5-m-long pile of Figure 2-6a (note that both the 5-m and 10-m piles have the same slenderness ratio). This indicates that the 
10-m-long pile in clay does not behave as a long pile and that the depth of fixity $h_{f}$ is greater than the length of the pile. Thus, the greater the ratio of pile to soil stiffness is, the larger the depth of fixity is and the larger the length required for a pile to behave as a long pile is.

For long piles, the solution given by equation (2-40) must be modified. This is required because, for long piles, the exponential terms with positive exponents (i.e., the terms containing $e^{a \tilde{z}}$ or $\left.e^{b \tilde{z}}\right)$ in the functions $\Phi_{1}, \Phi_{2}, \Phi_{3}$ and $\Phi_{4}$ (Table 2-1) become excessively large for sufficiently large $\tilde{z}$ (note that, the stiffer the soil, the greater the values of $k, t, a$ and $b$ are) and the solution no longer represents the actual physical problem. The reason why this happens is that, for long piles, the exponential terms with negative exponents (i.e., the terms containing $e^{-a \tilde{z}}$ or $e^{-b \tilde{z}}$ ) become negligible beyond a threshold value of $\tilde{z}$ and cannot balance the effects of the extremely large positive exponential terms. This makes the general solution unacceptable because the pile deflection, and thus the functions $\Phi_{1}, \Phi_{2}, \Phi_{3}$ and $\Phi_{4}$, should decrease with increasing $\tilde{z}$, while the positive exponential terms produce the opposite result.

The solution of equation (2-16b) or of equation (2-17b) for $\tilde{k_{i}}>\tilde{t}_{i}^{2}$, when rewritten by uncoupling the positive and negative exponential terms of the hyperbolic functions (Table 2-1), leads to:

$$
\tilde{w}_{i}(\tilde{z})=e^{-a \tilde{z}}\left[C_{1}^{(i)} \cos b \tilde{z}+C_{2}^{(i)} \sin b \tilde{z}\right]+e^{a \tilde{z}}\left[C_{3}^{(i)} \cos b \tilde{z}+C_{4}^{(i)} \sin b \tilde{z}\right]
$$

But, as $\tilde{z} \rightarrow 1\left(z \rightarrow L_{p}\right), \widetilde{w}_{i} \rightarrow 0$ (i.e., as the depth $z$ gets closer to the pile base, the deflection decreases and approaches a zero value). This condition can be satisfied only if the constants $C_{3}{ }^{(i)}$ and $C_{4}{ }^{(i)}$ are each identically equal to zero because, otherwise, the term $e^{a \tilde{z}}$ will dominate (i.e., it will increase greatly with increases in $\tilde{z}$ and produce large values of $\widetilde{w}_{i}$ ). Consequently, we get only two functions $\Phi_{1}$ and $\Phi_{2}$, associated with the negative exponent term, as the solutions for equation (2-16b) and for equation (2-17b) with $\tilde{k}_{i}>\tilde{t}_{i}^{2}$. The solution for equation $(2-17 \mathrm{~b})$ with $\tilde{k_{i}}<\tilde{t}_{i}^{2}$ can be similarly written by decoupling the positive and negative exponents as:

$$
\tilde{w}_{i}(\tilde{z})=C_{1}^{(i)} e^{-a \tilde{z}}+C_{2}^{(i)} e^{-b \tilde{z}}+C_{3}^{(i)} e^{a \tilde{z}}+C_{4}^{(i)} e^{b \tilde{z}}
$$

Following similar logic as for equation (2-100), the constants $C_{3}{ }^{(i)}$ and $C_{4}{ }^{(i)}$ in equation (2-101) are zero, and again we get only two functions which contain the negative exponential terms. Thus, for long piles, the general solution can be written as: 


$$
\widetilde{w}_{i}(\widetilde{z})=C_{1}^{(i)} \Phi_{1}+C_{2}^{(i)} \Phi_{2}
$$

The details of the functions $\Phi_{1}$ and $\Phi_{2}$ and their derivatives are given in Table 2-2.

The reduction of the solution functions from four to two can be explained using linear algebra as well. The arguments $a \tilde{z}$ or $b \tilde{z}$ of the hyperbolic functions in Table 2-1 have values greater than 3 for values of $\tilde{z}$ corresponding to depths greater than the depth of fixity. For such values of arguments, hyperbolic sine and cosine functions give almost identical results (i.e., the functions, when plotted, fall on top of each other for arguments greater than 3 ). Since functions can be treated as vectors in linear algebra, the solution $\widetilde{w}_{i}(\widetilde{z})=C_{1}^{(i)} \Phi_{1}+C_{2}^{(i)} \Phi_{2}+C_{3}^{(i)} \Phi_{3}+C_{4}^{(i)} \Phi_{4}$ can be assumed to represent a vector space (Strang 1988) with the linearly-independent vectors $\Phi_{1}, \Phi_{2}, \Phi_{3}$ and $\Phi_{4}$ (the functions $\Phi_{1}, \Phi_{2}, \Phi_{3}$ and $\Phi_{4}$ are linearly independent because they are solutions of linear differential equations) forming the basis of the four-dimensional vector space. However, when two of the functions become equal to the other two (i.e., $\sinh a \tilde{z}=\cosh a \tilde{z}$ and $\sinh b \tilde{z}=\cosh b \tilde{z})$, there are only two linearly-independent vectors, and the four-dimensional solution (vector) space collapses to a two-dimensional space. Consequently, we need only two functions to produce the required solution. 


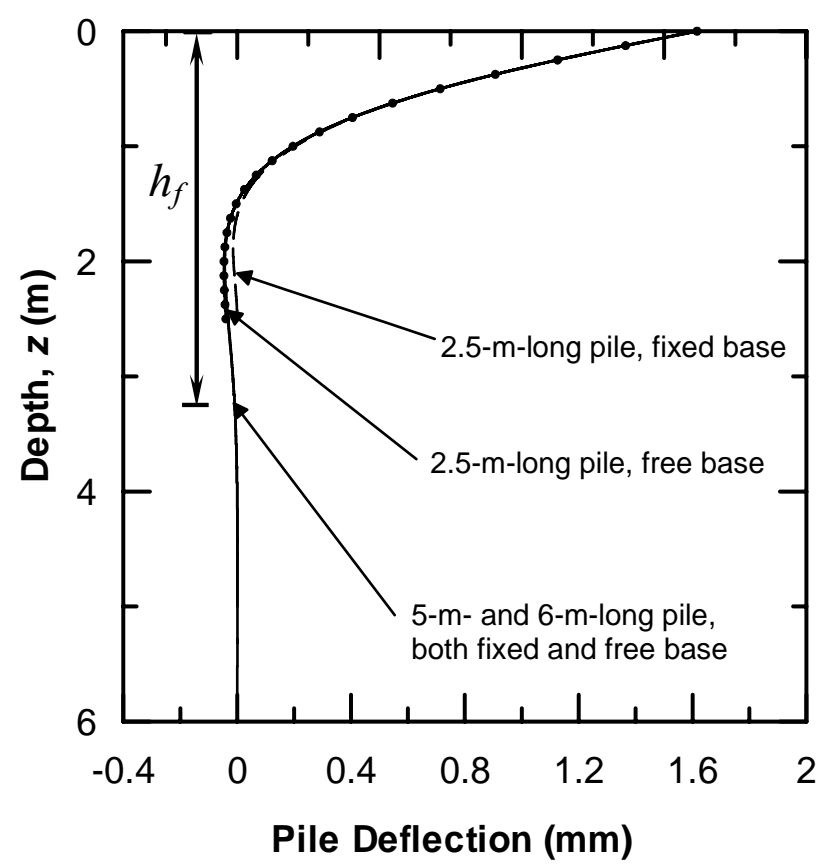

(a)

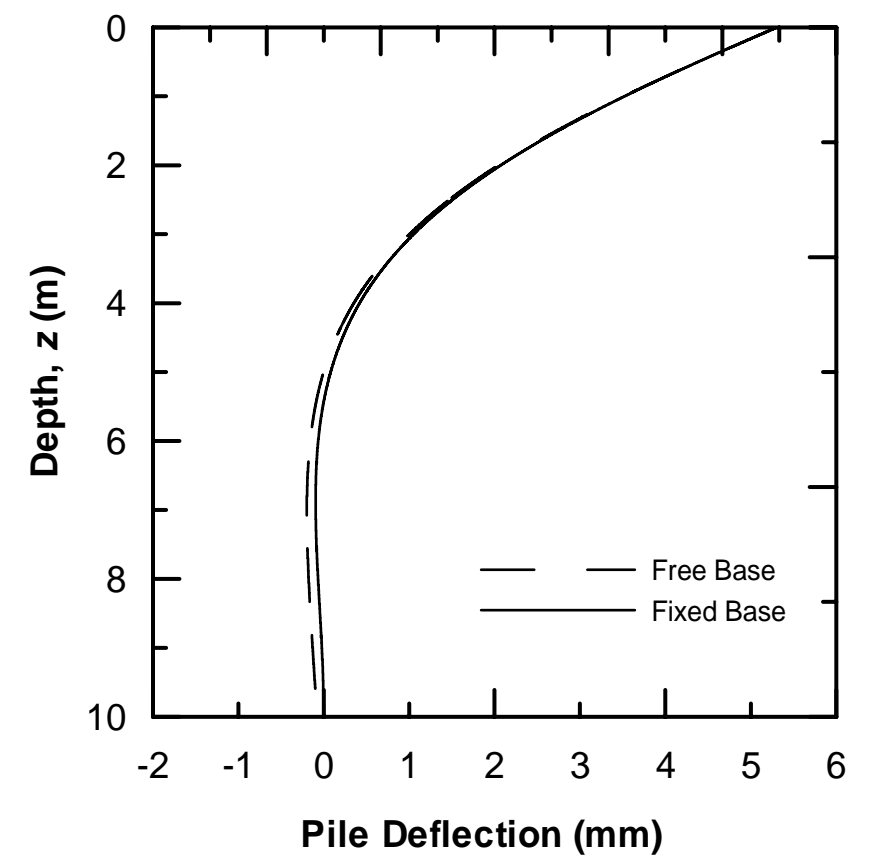

(b)

Figure 2-7 Piles in (a) Dense Sand and (b) Soft Clay 
Table 2-2 Functions in Equation (2-102) for Infinitely Long Piles Crossing Multiple Soil Layers

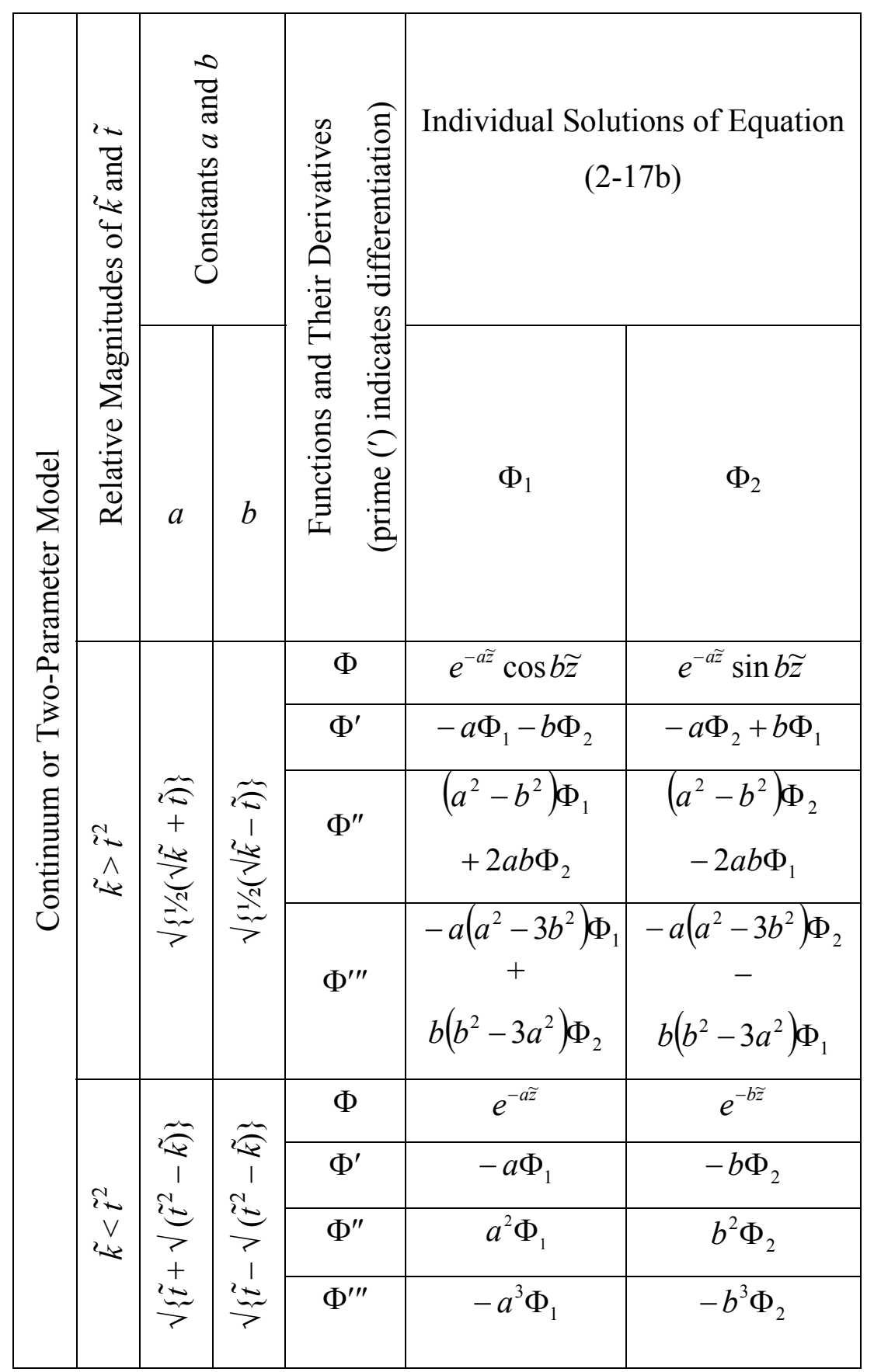

Note: For the individual solutions of equation (2-16b) (i.e., for the one-parameter model), the functions $\Phi_{1}$ and $\Phi_{2}$ are the same as for the case $\tilde{k}>\tilde{t}^{2}$ in Table 2-2, but $a=b=\sqrt[4]{\tilde{k} / 4}$. 


\subsubsection{Method of Initial Parameters}

Application of MIP to equation (2-102) requires modifications to the method described earlier, although the procedure, in principle, is the same. The solution procedure using MIP is simple in case of a homogeneous soil deposit; however, for layered deposits, MIP equations will be different depending on the location of the depth of fixity. For layers deeper than the depth of fixity $h_{f}$ (and, indeed, for portions of a layer deeper than it), the MIP equations are not the same as the equations derived earlier, which apply to $z<h_{f}$.

In order to perform the analysis, it is essential to know whether a pile falls under the category of long (infinite) pile or not (i.e., whether or not the depth of fixity is less than the pile length). Such studies for beams on elastic foundations (i.e., whether a beam on elastic foundation behaves as an infinite beam so that its boundary conditions do not affect its response) have been studied by several authors. For example, Hetényi (1946) suggested that, for a beam on a one-parameter foundation, if the length $l_{b}$ of the beam is greater than $\pi / \lambda_{b}$, where $\lambda_{b}=\sqrt[4]{k / 4 E_{b} I_{b}}$ ( $k$ is the soil spring constant per unit beam length, $E_{b}$ is the beam Young's modulus and $I_{b}$ is the second moment of inertia of beam section), then the beam behaves as an infinite beam. Vesić (1961), on the other hand, suggested that $l_{b}$ should be greater than $5 / \lambda_{b}$ for a beam on a one-parameter foundation to behave as an infinite beam. Similar limits are available for beams on two-parameter foundations as well (Vlasov and Leont'ev 1966). However, these limits were obtained by considering some specific examples (with specific loading conditions and specific values of foundation parameters) and comparing the responses of beams of different lengths for those specific conditions. Consequently, the limits suggested by these authors are applicable to some specific cases; the limits change with loading condition and foundation parameters. Moreover, these limits were obtained for infinite beams for which both the beam ends are at infinite distance from the applied loads, while a long laterally loaded pile behaves as a semi-infinite beam with only one end far away from the applied loads. Fleming et al. (1992) suggested that the depth of fixity of laterally loaded piles in a one-parameter foundation is $4 / \lambda_{p}$ where $\lambda_{p}=\sqrt[4]{k / 4 E_{p} I_{p}}$. Poulos and Davis (1980) suggested that the depth of fixity is $2.5 / \lambda_{p}$ and $1.5 / \lambda_{p}$ for free-head and fixed-head piles, respectively. Clearly, there is no consensus in the literature regarding the issue, and no limit for the depth of fixity for piles in two-parameter foundation is available. Additionally, these limits for beams and piles were proposed based on 
the assumption of a homogeneous deposit (i.e., with one value for $k$ and one value for $t$ throughout), while, in our case, we have a layered deposit. For these reasons, these limits, proposed for beams and piles, cannot be adapted in our study. The criterion adapted in this thesis to identify depths greater than the depth of fixity (i.e., to identify long piles) is the following: if, at any depth $z$ within a layer $i, a_{i} \tilde{z}>3$ or $b_{i} \tilde{z}>3$ (a value slightly higher but not greater than 3.5 can be used as well), then the depth of fixity corresponds to that depth $z$. Thus, piles longer than that depth behave as long piles. This criterion is mathematically rigorous, as explained before, and is easily applicable for layered media.

The MIP equations for equation (2-102) can be developed by following a similar procedure as was followed before for equation (2-40). Since there are only two constants now, only two initial parameters are required. For any layer $i$, the new MIP equations are given by:

$$
\begin{aligned}
& \tilde{w}_{i}(\tilde{z})=\tilde{w}_{0}^{(i)} K_{w w}^{(i)}+\tilde{\theta}_{0}^{(i)} K_{w \theta}^{(i)} \\
& \tilde{\theta}_{i}(\tilde{z})=\tilde{w}_{0}^{(i)} K_{\theta w}^{(i)}+\tilde{\theta}_{0}^{(i)} K_{\theta \theta}^{(i)} \\
& \tilde{M}_{i}(\tilde{z})=\tilde{w}_{0}^{(i)} K_{M w}^{(i)}+\tilde{\theta}_{0}^{(i)} K_{M \theta}^{(i)} \\
& \tilde{S}_{i}(\tilde{z})=\tilde{w}_{0}^{(i)} K_{S w}^{(i)}+\tilde{\theta}_{0}^{(i)} K_{S \theta}^{(i)}
\end{aligned}
$$

In order to illustrate how MIP works for long piles, we will again consider the same example of a three-layer soil (Figure 2-5) with $\tilde{k_{i}}>\tilde{t}_{i}^{2}$ for all the layers. The pile head is assumed to be free; however, no condition for pile base is required to be stated explicitly because, for long piles, the base is so far away from the influence of the applied force or moment that the deflection, slope, bending moment and shear force at the base are all equal to zero. We will further assume that the depth of fixity lies in the second (middle) layer. In other words, the arguments $a \tilde{z}$ and $b \tilde{z}$ become greater than 3 in the second layer. Thus, the MIP equations corresponding to the top layer, as described before in the example, are all valid, while, for the second and the third (bottom) layers new equations need to be developed.

A point to be noted here is that, although $a \tilde{z}$ and $b \tilde{z}$ are assumed to become greater than 3 in the middle layer in this example, they are not necessarily greater than 3 in the bottom layer (this can happen if the bottom layer consists of soft or loose soil so that the values of $a$ and $b$ are lower than 3). However, equation (2-102) is still valid for the bottom layer (even if $a \tilde{z}$ and $b \tilde{z}$ are less than 3 ) because, once the depth of fixity is reached for any layer, deflections can only 
decrease with further increase in $\tilde{z}$ (which means that the positive exponential terms should be omitted).

As before, we first choose the initial sections for each soil layer. For the top layer, the pile head is chosen as the initial section. For the bottom layer, the pile base cannot be chosen as the initial section because the corresponding initial parameters (i.e., the deflection and slope at pile base) are already known to be zero. So, for both the middle and the bottom layers, we choose the upper interfaces as the initial sections. Thus, the initial sections in the example are at $\tilde{z}=0$ for the top layer, at $\tilde{z}=\tilde{H}_{1}$ for the middle layer and at $\tilde{z}=\tilde{H}_{2}$ for the bottom layer. The initial parameters are $\tilde{w}_{0}^{(1)}, \tilde{\theta}_{0}^{(1)}, \tilde{M}_{0}^{(1)}$ and $\tilde{S}_{0}^{(1)}$ for the top layer, $\tilde{w}_{0}^{(2)}$ and $\tilde{\theta}_{0}^{(2)}$ for the middle layer, and $\tilde{w}_{0}^{(3)}$ and $\tilde{\theta}_{0}^{(3)}$ for the bottom layer.

Next, we impose the boundary conditions. Imposing boundary conditions at the pile head results in equations (2-63) and (2-64). This leads to equations (2-67) through (2-70) again describing the top layer. The pile base boundary conditions have already been used up in obtaining equation (2-102). Using the interface boundary conditions at $\tilde{H}_{1}$ and $\tilde{H}_{2}$, we get the following matrix equation:

$$
\left[\begin{array}{cccccc}
K_{w w}^{(1)} & K_{w \theta}^{(1)} & -1 & 0 & 0 & 0 \\
K_{\theta w}^{(1)} & K_{\theta \theta}^{(1)} & 0 & -1 & 0 & 0 \\
K_{M w}^{(1)} & K_{M \theta}^{(1)} & -K_{M w}^{(2)} & -K_{M \theta}^{(2)} & 0 & 0 \\
K_{S w}^{(1)} & K_{S \theta}^{(1)} & -K_{S w}^{(2)} & -K_{S \theta}^{(2)} & 0 & 0 \\
0 & 0 & K_{w w}^{(2)} & K_{w \theta}^{(2)} & -1 & 0 \\
0 & 0 & K_{\theta w}^{(2)} & K_{\theta \theta}^{(2)} & 0 & -1
\end{array}\right]\left[\begin{array}{c}
\tilde{w}_{0}^{(1)} \\
\tilde{\theta}_{0}^{(1)} \\
\tilde{w}_{0}^{(2)} \\
\tilde{\theta}_{0}^{(2)} \\
\tilde{w}_{0}^{(3)} \\
\tilde{\theta}_{0}^{(3)}
\end{array}\right]=\left[\begin{array}{c}
-\tilde{M}_{a} K_{w M}^{(1)}-\tilde{F}_{a} K_{w S}^{(1)} \\
-\tilde{M}_{a} K_{\theta M}^{(1)}-\tilde{F}_{a} K_{\theta S}^{(1)} \\
-\tilde{M}_{a} K_{M M}^{(1)}-\tilde{F}_{a} K_{M S}^{(1)} \\
-\tilde{M}_{a} K_{S M}^{(1)}-\tilde{F}_{a} K_{S S}^{(1)} \\
0 \\
0
\end{array}\right]
$$

In the above matrix equation, the first four rows are valid at $\tilde{z}=\tilde{H}_{1}$ while the remaining two are valid at $\tilde{z}=\tilde{H}_{2}$.

The influence coefficients can be obtained as before. Equations (2-76) through (2-91) give the influence coefficients for the top layer. In order to obtain the influence coefficients for the middle (second) layer, we refer back to equation (2-102). The normalized pile deflection, slope, bending moment and shear force can be obtained from equation (2-102) and its derivatives (see Table 2-2 under $\tilde{k}>\tilde{t}^{2}$ for the differentiations of $\Phi_{1}$ and $\Phi_{2}$ ) as:

$$
\begin{aligned}
& \widetilde{w}_{2}=C_{1} \Phi_{1}+C_{2} \Phi_{2} \\
& \widetilde{\theta}_{2}=-C_{1}\left(a \Phi_{1}+b \Phi_{2}\right)+C_{2}\left(-a \Phi_{2}+b \Phi_{1}\right)
\end{aligned}
$$




$$
\begin{aligned}
& \tilde{M}_{2}=C_{1}\left\{\left(a^{2}-b^{2}\right) \Phi_{1}+2 a b \Phi_{2}\right\}+C_{2}\left\{\left(a^{2}-b^{2}\right) \Phi_{2}-2 a b \Phi_{1}\right\} \\
& \widetilde{S}_{2}=C_{1}\left\{-a\left(a^{2}-3 b^{2}\right) \Phi_{1}+b\left(b^{2}-3 a^{2}\right) \Phi_{2}\right\}+C_{2}\left\{-a\left(a^{2}-3 b^{2}\right) \Phi_{2}-b\left(b^{2}-3 a^{2}\right) \Phi_{1}\right\} \\
& \quad-2 \widetilde{t}_{1}\left\{-C_{1}\left(a \Phi_{1}+b \Phi_{2}\right)+C_{2}\left(-a \Phi_{2}+b \Phi_{1}\right)\right\}
\end{aligned}
$$

where the constants $C_{1}$ and $C_{2}$ are valid for the second layer.

We solve equations (2-108) and (2-109) for $C_{1}$ and $C_{2}$ at the initial section of the second layer (i.e., at $\tilde{z}=\tilde{H}_{1}$ ):

$$
\begin{aligned}
& \widetilde{w}_{0}^{(2)}=C_{1} \phi_{1}+C_{2} \phi_{2} \\
& \widetilde{\theta}_{0}^{(2)}=-C_{1}\left(a \phi_{1}+b \phi_{2}\right)+C_{2}\left(-a \phi_{2}+b \phi_{1}\right)
\end{aligned}
$$

where $\phi_{1}$ and $\phi_{2}$ are the values of $\Phi_{1}$ and $\Phi_{2}$ (Table 2-2) at $\tilde{z}=\tilde{H}_{1}$. Solving equations (2-112) and (2-113), we get:

$$
\begin{aligned}
& C_{1}=\frac{\tilde{w}_{0}^{(2)}\left(b \phi_{1}-a \phi_{2}\right)-\tilde{\theta}_{0}^{(2)} \phi_{2}}{b\left(\phi_{1}^{2}+\phi_{2}^{2}\right)} \\
& C_{2}=\frac{\tilde{w}_{0}^{(2)}\left(a \phi_{1}+b \phi_{2}\right)+\tilde{\theta}_{0}^{(2)} \phi_{1}}{b\left(\phi_{1}^{2}+\phi_{2}^{2}\right)}
\end{aligned}
$$

Substituting the constants $C_{1}$ and $C_{2}$ into equations (2-108) through (2-111) and comparing the coefficients of $\tilde{w}_{0}^{(2)}$ and $\tilde{\theta}_{0}^{(2)}$ in these four equations with those in equations (2103) through (2-106) (with $i=2)$, we obtain the influence coefficients for the middle layer. The influence coefficients for the bottom layer can be obtained following similar steps, and, in fact, the expressions are identical to those of the middle layer (with the exception that $\phi_{1}$ and $\phi_{2}$ are the values of $\Phi_{1}$ and $\Phi_{2}$ at $\tilde{z}=\tilde{H}_{2}$ ).

Using the values of the influence coefficients, the unknown initial parameters are determined by solving equation (2-107). The initial parameters and the influence coefficients are then used to find the normalized pile deflection, slope, bending moment and shear force at any pile section by using equations (2-67) through (2-70) for layer 1 and by using equations (2-103) through (2-106) for layers 2 (with $i=2$ ) and 3 (with $i=3$ ), respectively.

The influence coefficients and matrix equations for up to four layers, with the depth of fixity lying in the first, second, third or fourth layers have been obtained for free or fixed pile head conditions and can be readily used. 
MIP can also be used to solve nonlinear problems by the recurring use of the MIP equations with different $k$ and $t$ values (when the relationships of $k$ and $t$ versus deflection or strain are known), until convergence is attained. This is illustrated in chapter 8. So the theory we present in this chapter can be the basis for nonlinear analyses that will be capable of handling very realistic problems.

\subsection{Example}

One example problem is solved for a laterally loaded pile in a four-layer soil deposit. The pile has a length $L_{p}=20.0 \mathrm{~m}$, radius $r_{p}=0.3 \mathrm{~m}$ and modulus $E_{p}=25 \times 10^{6} \mathrm{kN} / \mathrm{m}^{2}$ and is acted upon by a lateral force $F_{a}=300 \mathrm{kN}$ and a moment $M_{a}=100 \mathrm{kNm}$ at the pile head. The pile is assumed to be free both at the head and at the base. The soil deposit has four layers with $H_{1}=5 \mathrm{~m}, H_{2}=10 \mathrm{~m}$ and $H_{3}=15 \mathrm{~m}$. A two-parameter or continuum model with $k_{1}=56.0 \mathrm{MPa}$, $k_{2}=140.0 \mathrm{MPa}, k_{3}=155.0 \mathrm{MPa}$ and $k_{4}=200.0 \mathrm{MPa}$, and $t_{1}=11.0 \mathrm{MN}, t_{2}=28.0 \mathrm{MN}, t_{3}=40.0$ $\mathrm{MN}$ and $t_{4}=60.0 \mathrm{MN}$ is assumed. Figure 2-8(a), (b), (c) and (d) show the pile deflection, bending moment, shear force and soil resistance, respectively. Note that the depth of fixity is at about $8 \mathrm{~m}$. 


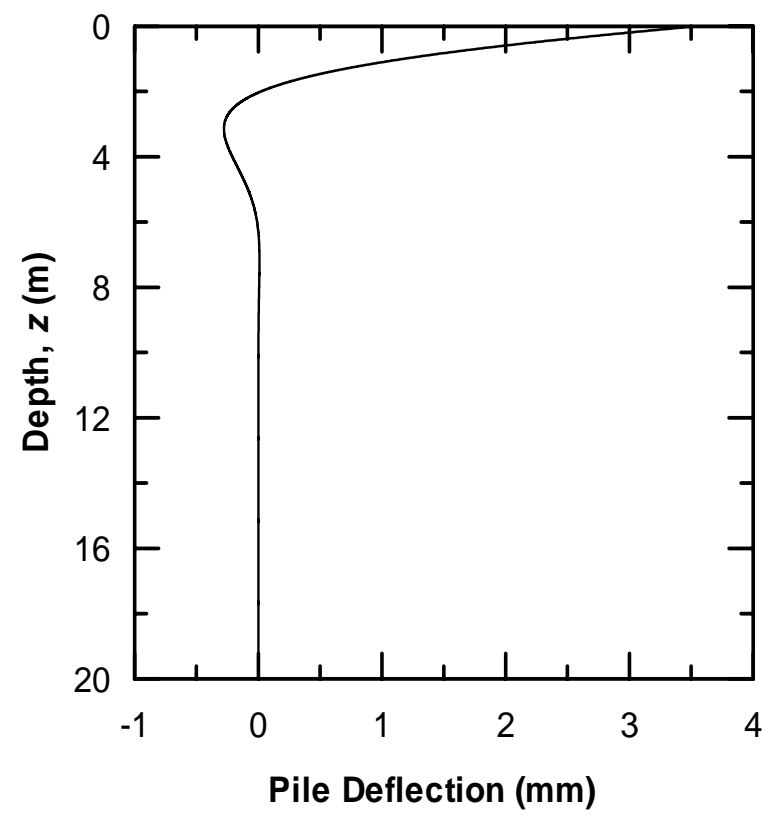

(a)

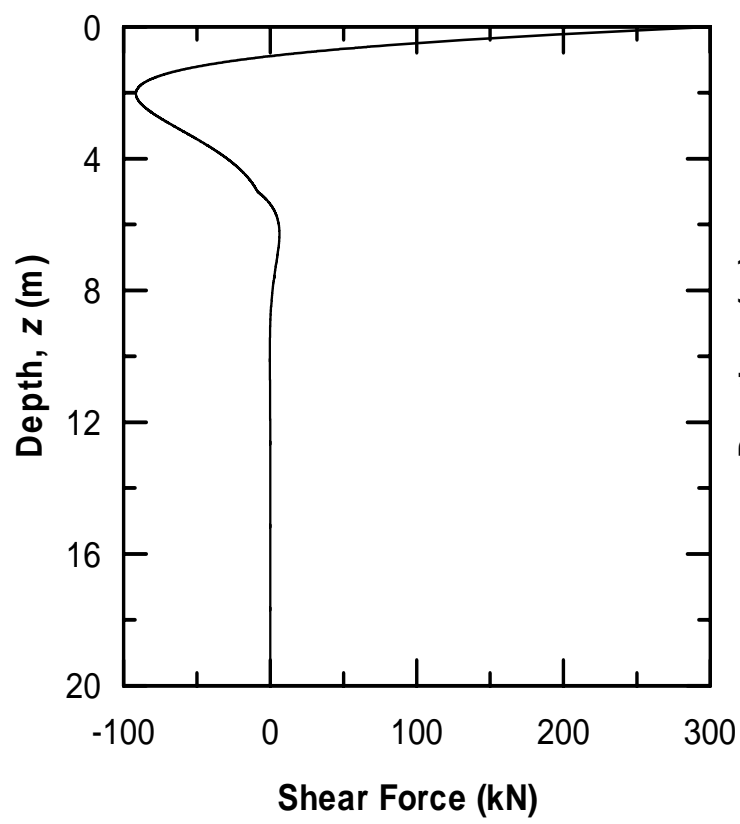

(c)

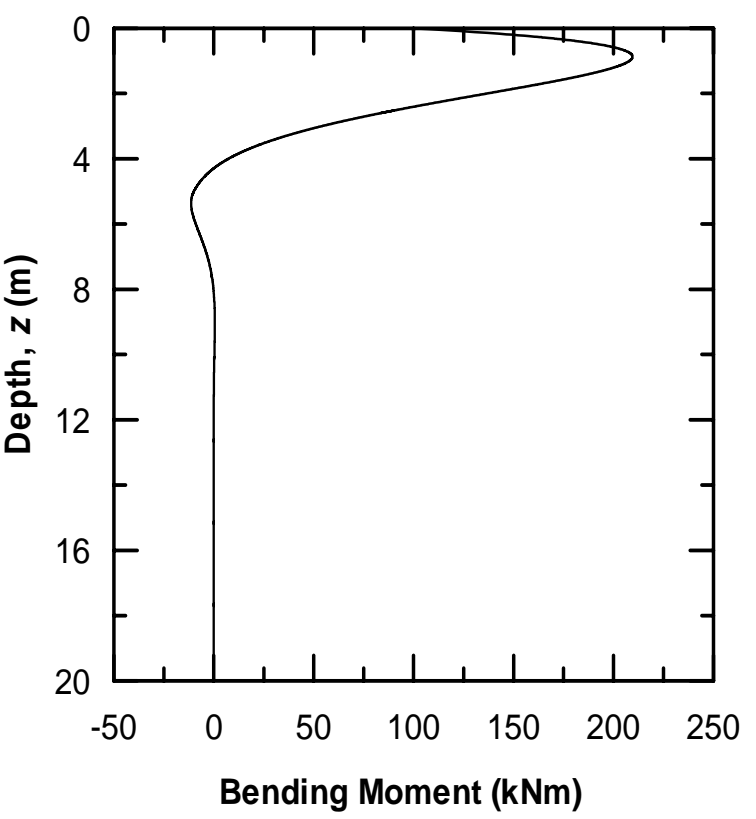

(b)

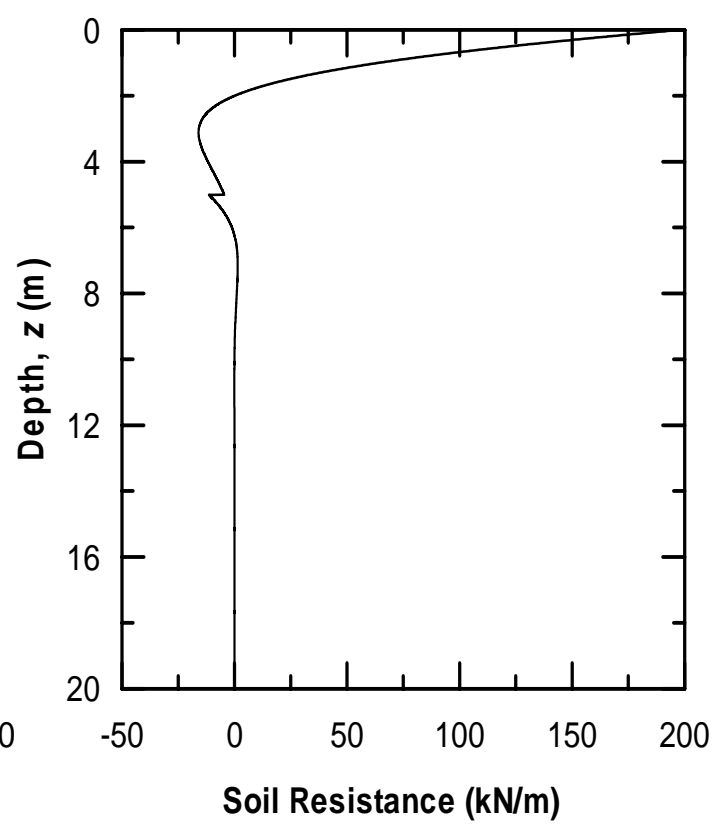

(d)

Figure 2-8 (a) Deflection, (b) Bending Moment, (c) Shear Force and (d) Soil Resistance of a Laterally Loaded Pile

\subsection{Summary}

The governing differential equations and boundary conditions for laterally loaded piles embedded in layered elastic media were developed following a beam-on-elastic-foundation 
approach. The method of initial parameters, traditionally used to solve problems of beams on elastic foundations, was then modified to obtain analytically the pile deflection, slope, bending moment and shear force as functions of depth. The method was illustrated using an example of a laterally loaded pile embedded in a three-layer soil deposit. 


\section{CHAPTER 3. CONTINUUM ANALYSIS OF LATERALLY LOADED PILE IN LAYERED ELASTIC MEDIUM}

\subsection{Introduction}

In this chapter, we develop a method of analysis for a laterally loaded pile embedded in a multi-layered soil deposit by treating the soil deposit as a three-dimensional, elastic continuum. We apply the principle of minimum potential energy to obtain the governing differential equations for deflection of pile and displacements in the continuum, after making some simplified assumption regarding the displacement field within the elastic medium. The resulting differential equations describing the pile deflection and soil displacements are interdependent, showing that the analysis takes explicit account of the soil-structure interaction.

The differential equations governing the pile deflection function and the boundary conditions are exactly the same as the corresponding equations obtained for the two-parameter foundation model in chapter 2. Thus, we show a one-to-one correspondence between the continuum-based approach and the beam-on-elastic-foundation approach for laterally loaded piles in layered media. Moreover, the analysis developed in this chapter provides a rational basis for the calculation of the parameters $k$ and $t$ for the elastic foundation models described in chapter 2. Because of the correspondence between the two approaches, analytical solutions of the pile deflection equations developed in this chapter can be obtained using the MIP, which was described in chapter 2 .

\section{2. $\underline{\text { Overview }}$}

The beam-on-elastic foundation approach, outlined in chapter 2, can be efficiently used for the analysis of laterally loaded piles in elastic soil if we can readily obtain the values of the parameters $k$ and $t$ for different soils. The spring constant $k$ can be determined from plate load tests; but the limitation is that the obtained values of $k$ are not unique because the values depend on many factors like the size, shape and flexibility of the plate, depth at which the test is 
performed and the rate of loading (Selvadurai 1979). Moreover, a plate load test determines the vertical stiffness of ground, whereas, for laterally loaded piles, horizontal stiffness is required. A few laterally loaded plate tests were performed by Georgiadis and Butterfield (1982) to obtain both the parameters $k$ and $t$ (and their nonlinear variation with pile deflection) by fitting a twoparameter foundation-model equation, proposed by Kerr (1964), to the experimental results. However, performing such tests for routine projects is uneconomical and is not used in practice. Efforts have been made to relate results of the standard penetration test (SPT) and the cone penetration test (CPT) with the modulus of subgrade reaction (Anderson and Townsend 2001). The pressuremeter test and the dilatometer test can also be used to estimate $k$; in fact, they have been used to develop p-y curves (Gabr et al. 1994, Briaud 1997). But such correlations are empirical and not generally applicable. Thus, there is no easy and rational way of directly determining the value of $k$ or $t$ that is applicable for laterally loaded pile analysis. In fact, most researchers neglect the shear parameter (i.e., assume a one-parameter model). Some authors, based on experience and back calculation of numerical analyses, have proposed some bulk-part values (or range of values) of $k$ for different soils (Poulos and Davis 1980, Scott 1981, Bowles 1997). Similar empirical approach is adopted to develop the p-y curves as well (Reese and Cox 1969, Matlock 1970, Reese et al. 1974, 1975).

Clearly, improvements in estimating the soil parameters are necessary if realistic predictions for lateral deflections of piles are to be made. Such an effort for beams on elastic foundations led several authors to develop empirical and semi-empirical relationships between the parameter $k$ and the elastic constants of the soil, namely, Young's modulus $E_{S}$ and Poisson's ration $v_{s}$ (Vesić 1961, Biot 1937). The advantage of this approach is that the elastic constants can be determined with reasonable accuracy from a variety of laboratory and field tests or through simple correlations with easily measurable soil properties (Salgado 2008, Bowles 1997, Selvadurai 1979), and typical values (or range of values) of the constants for different types of soil are available in the literature (Selvadurai 1979, Rao 1998, Bowles 1997). However, the available relationships relating $k$ with $E_{s}$ and $v_{s}$ for beams are semi-empirical, and although modifications of these expressions for laterally piles are available (Francis 1964, Hsiung and Chen 1997, Ashford and Juirnarongrit 2003), these relationships are restrictive in their applicability and not rigorous enough to be used reliably in design. 
A rational method of analysis of beams on elastic foundations was developed by Vlasov and Leont'ev (1966) based on an elastic continuum approach. Their analysis rigorously related the two parameters $k$ and $t$ with the soil elastic constants $E_{s}$ and $v_{s}$ using variational principles. These relationships are valid for any generalized loading and for all boundary conditions. In their analysis, Vlasov and Leont'ev (1966) considered only vertical displacement within the continuum (displacements in the horizontal directions were assumed to be zero) and expressed the displacement as a product of two separable functions; an unknown function describing the beam deflection and a known function describing the variation of displacement within the continuum. Applying the principle of virtual work, Vlasov and Leont'ev (1966) obtained the governing differential equation for the beam deflection function, which is exactly the same as the equation for the two-parameter foundation. Improvement to this analysis was done by Rao et al. (1971), who considered non-zero horizontal displacement in the foundation.

Vlasov and Leont'ev (1966) had assumed a known function that described the variation of displacement with depth within the continuum. Vallabhan and Das (1988), (1991), following a methodology adapted by Jones and Xenophontos (1977) for analyzing plates on elastic foundations, improved the analysis of Vlasov and Leont'ev (1966) by letting the spatial function be unknown to begin with, and then rigorously determined the function using variational principles. This analysis framework was adapted by Sun (1993), (1994a), who applied it to laterally loaded piles embedded in homogeneous elastic media. Guo and Lee (2001) modified the analysis by assuming a simplified stress field in the continuum surrounding the pile; however, such simplification led to empirical equations relating $k$ and $t$ with $E_{s}$ and $v_{s}$. Yang et al. (2002) extended the analysis of Sun (1994a) for a two-layer system; however, the boundarycondition equations pertaining to shear force were incorrect, making the analysis unusable.

In the analysis of Sun (1994a), the assumption regarding the displacement field, that the variation of displacements within the soil mass depends on the same displacement function for both the radial and circumferential directions, leads to a soil response that is stiffer than it is in reality. In this chapter, we adapt the basic methodology of Sun (1994a) but propose a new displacement field by assuming different displacement functions for the radial and circumferential directions that removes the artificial stiffness in the model of Sun (1994a). At the same time, we extend the analysis to account for multi-layered soil. A similar attempt to 
remove the artificial stiffness was made by Vallabhan (1999); however, the expressions obtained in that paper are valid only for a single layer.

This analysis allows us to rationally link $k_{i}$ and $t_{i}$ with the Young's modulus $E_{s i}$ and Poisson's ratio $v_{s i}$ for any layer $i$. It also shows how the resistive properties of soil elements (i.e., the stress-strain relationships) add together to produce the overall soil resistance against laterally loaded pile movement. At the same time, the analysis forms the basis for future formulations that can take into account the nonlinear stress-strain behavior of soil.

\subsection{Analysis}

\subsubsection{Problem Definition}

We consider a pile with a circular cross section of radius $r_{p}$ and length $L_{p}$ embedded in a soil deposit that has $n$ layers (Figure 3-1). Each layer extends to infinity in all radial directions, and the bottom $\left(n^{\text {th }}\right)$ layer extends to infinity in the downward direction. The vertical depth to the base of any intermediate layer $i$ is $H_{i}$, which implies that the thickness of the $i^{\text {th }}$ layer is $H_{i}-$ $H_{i-1}$ with $H_{0}=0$. The pile head is at the ground surface, and the base is embedded in the $n^{\text {th }}$ layer. The pile is subjected to a horizontal force $F_{a}$ and a moment $M_{a}$ at the pile head such that $F_{a}$ and $M_{a}$ are orthogonal vectors.

In the analysis, we choose a cylindrical $(r-\theta-z)$ coordinate system with its origin coinciding with the center of the pile head and the positive $z$-axis (coinciding with the pile axis) pointing downward. The goal of the analysis is to obtain pile deflection as a function of depth caused by the action of $F_{a}$ and/or $M_{a}$ at the pile head.

The soil medium is assumed to be isotropic, homogeneous within each layer, and linearly elastic with Lame's constants $\lambda_{s}$ and $G_{s}$. There is no slippage or separation between the pile and the surrounding soil or between the soil layers. The pile behaves as an Euler-Bernoulli beam with a constant flexural rigidity $E_{p} I_{p}$. 


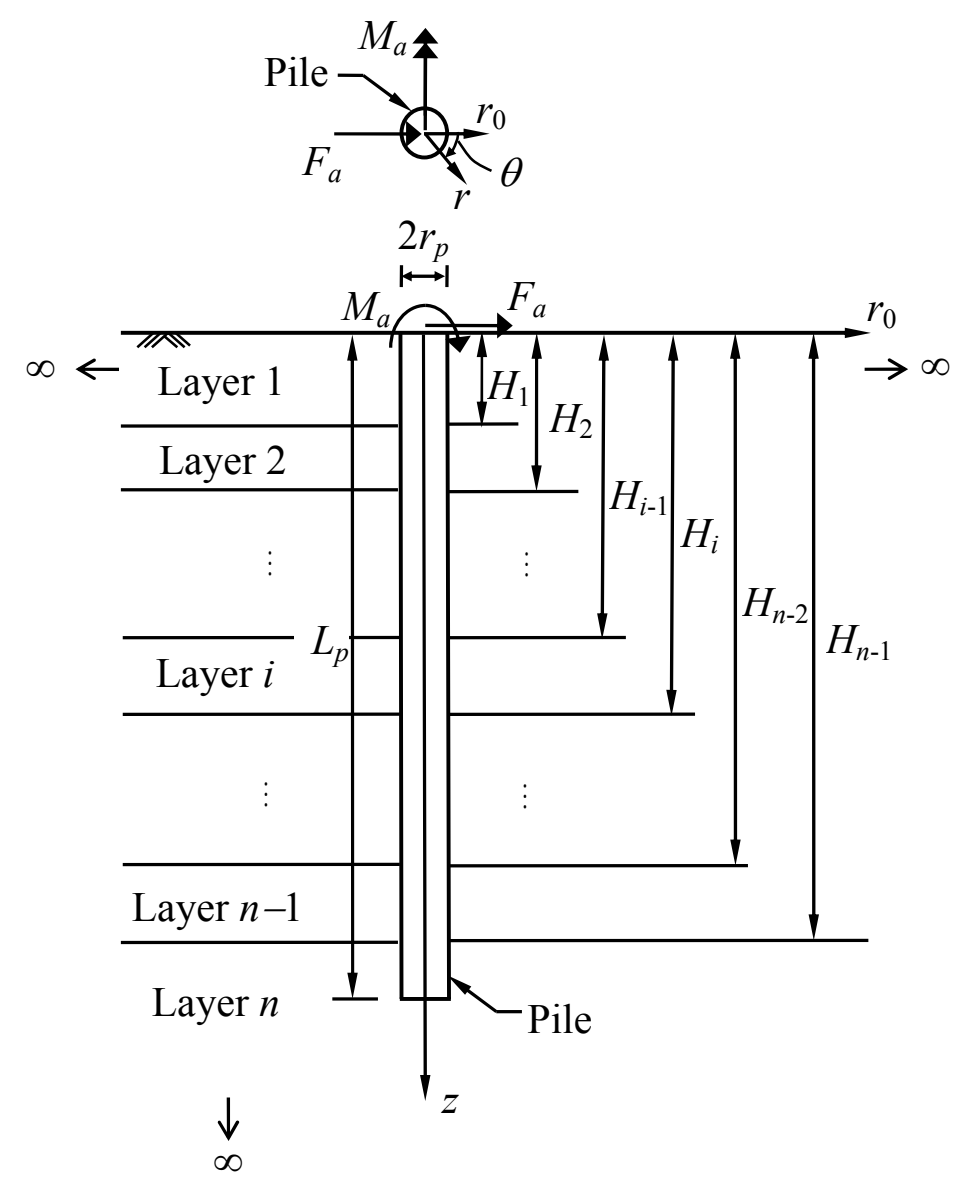

Figure 3-1 A Laterally Loaded Pile in a Layered Elastic Medium

\subsubsection{Potential Energy}

The total potential energy of the pile-soil system, including both the internal and external potential energies, is given by:

$$
\begin{aligned}
\Pi= & \frac{1}{2} E_{p} I_{p} \int_{0}^{L_{p}}\left(\frac{d^{2} w}{d z^{2}}\right)^{2} d z+\int_{0}^{\infty} \int_{0}^{2 \pi} \int_{r_{p}}^{\infty} \frac{1}{2} \sigma_{p q} \varepsilon_{p q} r d r d \theta d z+\int_{L_{p}}^{\infty} \int_{0}^{2 \pi} \int_{0}^{r_{p}} \frac{1}{2} \sigma_{p q} \varepsilon_{p q} r d r d \theta d z \\
& -\left.F_{a} w\right|_{z=0}+\left.M_{a} \frac{d w}{d z}\right|_{z=0}
\end{aligned}
$$

where $w$ is the lateral pile deflection; and $\sigma_{p q}$ and $\varepsilon_{p q}$ are the stress and strain tensors (see Figure $3-2$ ) in the soil (summation is implied by the repetition of the indices $p$ and $q$ in the product of corresponding stress and strain components). The first integral represents the internal potential energy of the pile. The second and third integrals represent the internal potential energy of the continuum (note that the third integral represents the energy of the column of soil with radius $r_{p}$ 
starting at the pile base and extending to infinity downward, while the second integral represents the energy of the soil surrounding both the pile and this column of soil). The remaining two terms represent the external potential energy.

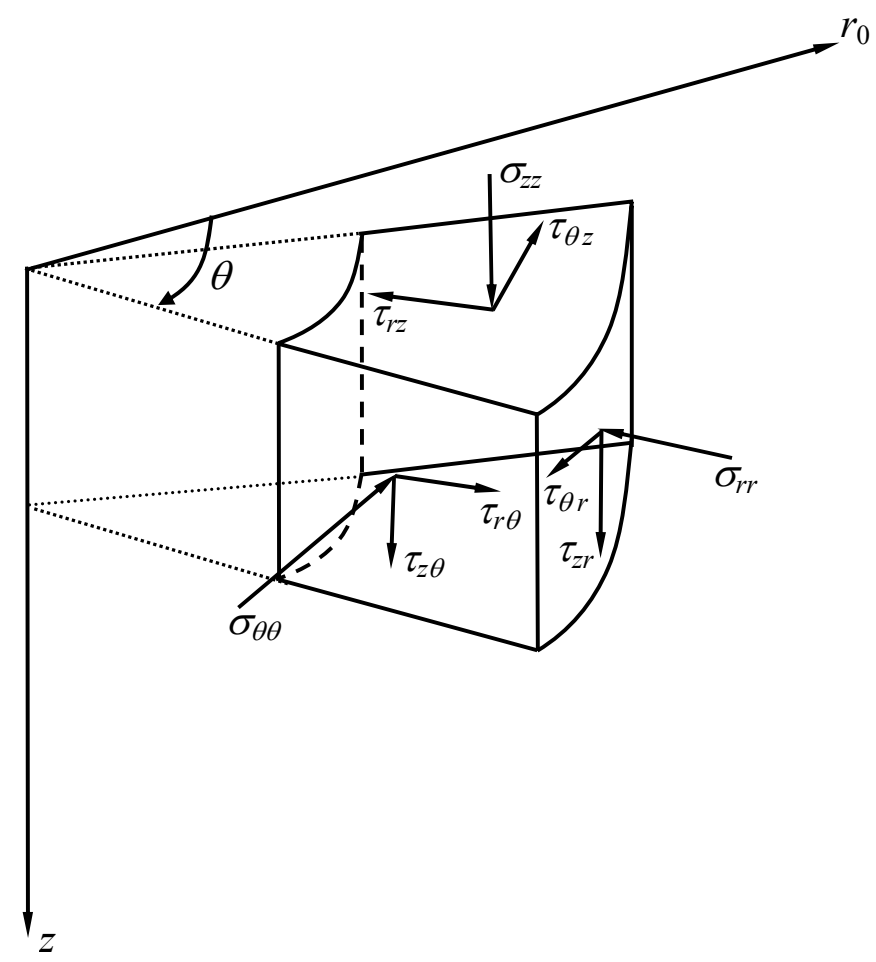

Figure 3-2 Stresses Within a Soil Continuum

\subsubsection{Displacement Field}

We assume the following displacement fields (Figure 3-3) in the soil:

$$
\begin{aligned}
& u_{r}=w(z) \phi_{r}(r) \cos \theta \\
& u_{\theta}=-w(z) \phi_{\theta}(r) \sin \theta \\
& u_{z}=0
\end{aligned}
$$

where $w(z)$ is a displacement function (with a dimension of length), varying with depth $z$, representing the deflection of the pile axis; $\phi_{r}(r)$ and $\phi_{\theta}(r)$ are dimensionless displacement functions varying with the radial coordinate $r$, and $\theta$ is the angle measured clockwise from a vertical reference section $\left(r=r_{0}\right)$ that contains the applied force vector $F_{a}$. Note that the 
reference plane $r_{0}-z$ coincides with the $x-z$ plane of the $x-y-z$ coordinate system (Figure 2-2) assumed in chapter 2. Equation (3-2c) is based on the assumption that the vertical displacement of the pile caused by the lateral load and moment applied at the pile head is negligible.

The functions $\phi_{r}(r)$ and $\phi_{\theta}(r)$ describe how the displacements within the soil mass (due to pile deflection) decrease with increasing radial distance from the pile axis. We set $\phi_{r}(r)=1$ and $\phi_{\theta}(r)=1$ at $r=r_{p}$ (this ensures compatibility at the pile-soil interface) and $\phi_{r}(r)=0$ and $\phi_{\theta}(r)=0$ at $r=\infty$ (this ensures that displacements in the soil decrease with increasing radial distance from the pile). Thus, $\phi_{r}$ and $\phi_{\theta}$ vary between 1 at the pile-soil interface to 0 at infinite radial distance from the pile.

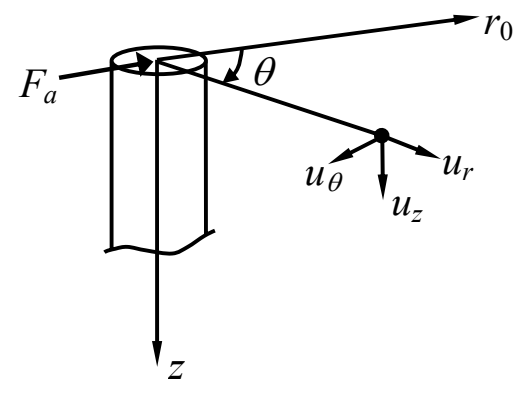

Figure 3-3 Displacements Within a Soil Continuum

\subsubsection{Stress-Strain-Displacement Relationships}

The strain-displacement relationship, considering equation (3-2), leads to:

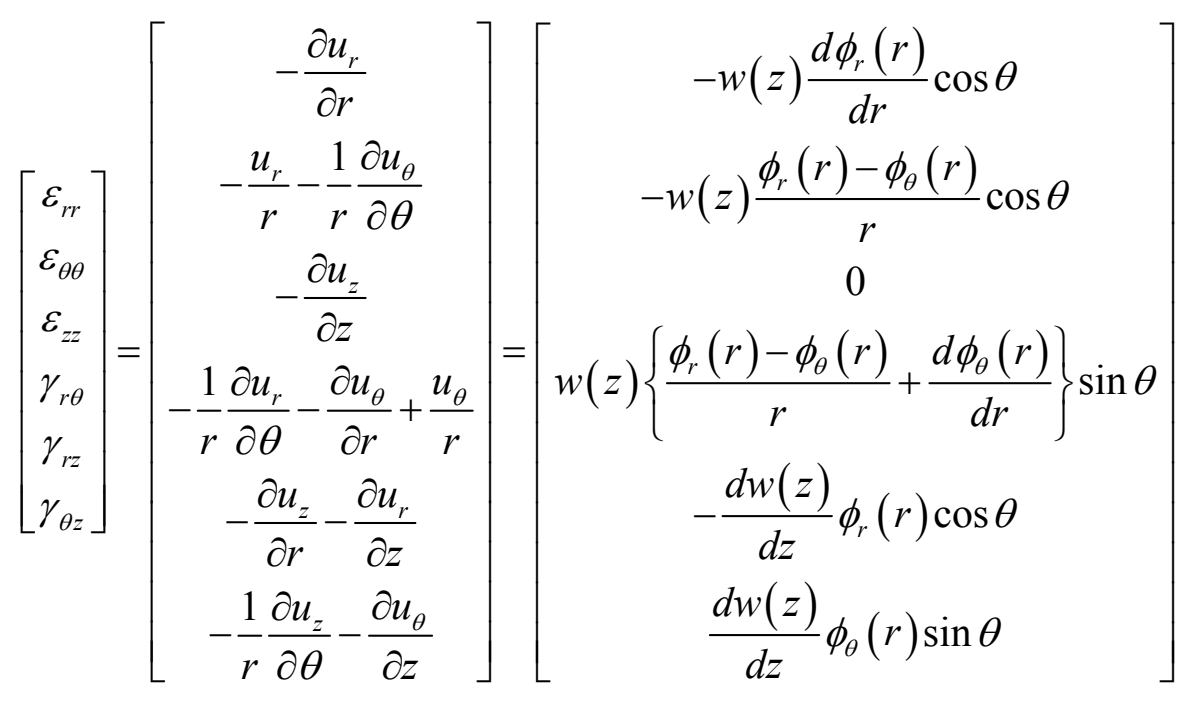


The stress-strain relationship in any elastic layer is given by:

$$
\left[\begin{array}{c}
\sigma_{r r} \\
\sigma_{\theta \theta} \\
\sigma_{z z} \\
\tau_{r \theta} \\
\tau_{r z} \\
\tau_{\theta z}
\end{array}\right]=\left[\begin{array}{cccccc}
\lambda_{s}+2 G_{s} & \lambda_{s} & \lambda_{s} & 0 & 0 & 0 \\
\lambda_{s} & \lambda_{s}+2 G_{s} & \lambda_{s} & 0 & 0 & 0 \\
\lambda_{s} & \lambda_{s} & \lambda_{s}+2 G_{s} & 0 & 0 & 0 \\
0 & 0 & 0 & G_{s} & 0 & 0 \\
0 & 0 & 0 & 0 & G_{s} & 0 \\
0 & 0 & 0 & 0 & 0 & G_{s}
\end{array}\right]\left[\begin{array}{c}
\varepsilon_{r r} \\
\varepsilon_{\theta \theta} \\
\varepsilon_{z z} \\
\gamma_{r \theta} \\
\gamma_{r z} \\
\gamma_{\theta z}
\end{array}\right]
$$

Combination of equations (3-3) and (3-4) gives the strain energy density within any layer as:

$$
\begin{aligned}
\frac{1}{2} \sigma_{p q} \varepsilon_{p q}=\frac{1}{2} & {\left[\left(\lambda_{s}+2 G_{s}\right) w^{2}\left(\frac{d \phi_{r}}{d r}\right)^{2} \cos ^{2} \theta+2 \lambda_{s} w^{2} \frac{d \phi_{r}}{d r} \frac{\left(\phi_{r}-\phi_{\theta}\right)}{r} \cos ^{2} \theta\right.} \\
& +\left(\lambda_{s}+G_{s}\right) w^{2} \frac{\left(\phi_{r}-\phi_{\theta}\right)^{2}}{r^{2}} \cos ^{2} \theta+G_{s} w^{2} \frac{\left(\phi_{r}-\phi_{\theta}\right)^{2}}{r^{2}} \\
& +G_{s} w^{2}\left(\frac{d \phi_{\theta}}{d r}\right)^{2} \sin ^{2} \theta+2 G_{s} w^{2} \frac{\left(\phi_{r}-\phi_{\theta}\right)}{r} \frac{d \phi_{\theta}}{d r} \sin ^{2} \theta \\
& \left.+G_{s}\left(\frac{d w}{d z}\right)^{2} \phi_{r}^{2} \cos ^{2} \theta+G_{s}\left(\frac{d w}{d z}\right)^{2} \phi_{\theta}^{2} \sin ^{2} \theta\right]
\end{aligned}
$$

Substituting equation (3-5) into equation (3-1), we get:

$$
\begin{aligned}
\Pi= & \frac{1}{2} E_{p} I_{p} \int_{0}^{L_{p}}\left(\frac{d^{2} w}{d z^{2}}\right)^{2} d z+\frac{\pi}{2} \int_{0}^{\infty} \int_{r_{p}}^{\infty}\left[\left(\lambda_{s}+2 G_{s}\right) w^{2}\left(\frac{d \phi_{r}}{d r}\right)^{2}\right. \\
& +2 \lambda_{s} w^{2} \frac{d \phi_{r}}{d r} \frac{\left(\phi_{r}-\phi_{\theta}\right)}{r}+\left(\lambda_{s}+3 G_{s}\right) w^{2} \frac{\left(\phi_{r}-\phi_{\theta}\right)^{2}}{r^{2}}+G_{s} w^{2}\left(\frac{d \phi_{\theta}}{d r}\right)^{2} \\
& \left.+2 G_{s} w^{2} \frac{\left(\phi_{r}-\phi_{\theta}\right)}{r} \frac{d \phi_{\theta}}{d r}+G_{s}\left(\frac{d w}{d z}\right)^{2} \phi_{r}^{2}+G_{s}\left(\frac{d w}{d z}\right)^{2} \phi_{\theta}^{2}\right] r d r d z \\
& +\frac{\pi}{2} r_{p}^{2} \int_{L_{p}}^{\infty} G_{s}\left(\frac{d w}{d z}\right)^{2} d z-\left.F_{a} w\right|_{z=0}+\left.M_{a} \frac{d w}{d z}\right|_{z=0}
\end{aligned}
$$

\subsubsection{Principle of Minimum Potential Energy}

A system in equilibrium exists with its potential energy at a minimum. Hence, minimizing the potential energy of the pile-soil system (i.e., setting the first variation $\delta \Pi$ of the 
potential energy equal to 0 ) produces the equilibrium equations. Applying $\delta \Pi=0$ to equation (3-6), we get:

$$
\begin{aligned}
& \left\{\int_{0}^{L_{p}} E_{p} I_{p} \frac{d^{2} w}{d z^{2}} \delta\left(\frac{d^{2} w}{d z^{2}}\right) d z+\pi \int_{0}^{\infty} \int_{r_{p}}^{\infty}\left[\left(\lambda_{s}+2 G_{s}\right) w \delta w\left(\frac{d \phi_{r}}{d r}\right)^{2}+2 \lambda_{s} w \delta w \frac{\phi_{r}}{r} \frac{d \phi_{r}}{d r}\right.\right. \\
& -2 \lambda_{s} w \delta w \frac{\phi_{\theta}}{r} \frac{d \phi_{r}}{d r}+\left(\lambda_{s}+3 G_{s}\right) w \delta w \frac{\phi_{r}^{2}}{r^{2}}+\left(\lambda_{s}+3 G_{s}\right) w \delta w \frac{\phi_{\theta}^{2}}{r^{2}} \\
& -2\left(\lambda_{s}+3 G_{s}\right) w \delta w \frac{\phi_{r} \phi_{\theta}}{r^{2}}+G_{s} w \delta w\left(\frac{d \phi_{\theta}}{d r}\right)^{2}+2 G_{s} w \delta w \frac{\phi_{r}}{r} \frac{d \phi_{\theta}}{d r} \\
& \left.-2 G_{s} w \delta w \frac{\phi_{\theta}}{r} \frac{d \phi_{\theta}}{d r}+G_{s} \frac{d w}{d z} \delta\left(\frac{d w}{d z}\right) \phi_{r}^{2}+G_{s} \frac{d w}{d z} \delta\left(\frac{d w}{d z}\right) \phi_{\theta}^{2}\right] r d r d z \\
& \left.+\pi r_{p}^{2} \int_{L_{p}}^{\infty} G_{s} \frac{d w}{d z} \delta\left(\frac{d w}{d z}\right) d z-\left.F_{a} \delta w\right|_{z=0}+\left.M_{a} \delta\left(\frac{d w}{d z}\right)\right|_{z=0}\right\} \\
& +\left\{\pi \int _ { 0 } ^ { \infty } \int _ { r _ { p } } ^ { \infty } \left[\left(\lambda_{s}+2 G_{s}\right) w^{2}\left(\frac{d \phi_{r}}{d r}\right) \delta\left(\frac{d \phi_{r}}{d r}\right)+\lambda_{s} w^{2} \frac{1}{r} \delta \phi_{r} \frac{d \phi_{r}}{d r}+\lambda_{s} w^{2} \frac{\phi_{r}}{r} \delta\left(\frac{d \phi_{r}}{d r}\right)\right.\right. \\
& -\lambda_{s} w^{2} \frac{\phi_{\theta}}{r} \delta\left(\frac{d \phi_{r}}{d r}\right)+\left(\lambda_{s}+3 G_{s}\right) w^{2} \frac{\phi_{r}}{r^{2}} \delta \phi_{r}-\left(\lambda_{s}+3 G_{s}\right) w^{2} \delta \phi_{r} \frac{\phi_{\theta}}{r^{2}} \\
& \left.\left.+G_{s} w^{2} \frac{1}{r} \delta \phi_{r} \frac{d \phi_{\theta}}{d r}+G_{s}\left(\frac{d w}{d z}\right)^{2} \phi_{r} \delta \phi_{r}\right] r d r d z\right\} \\
& +\left\{\pi \int_{0}^{\infty} \int_{r_{p}}^{\infty}-\lambda_{s} w^{2} \frac{1}{r} \delta \phi_{\theta} \frac{d \phi_{r}}{d r}+\left(\lambda_{s}+3 G_{s}\right) w^{2} \frac{\phi_{\theta}}{r^{2}} \delta \phi_{\theta}-\left(\lambda_{s}+3 G_{s}\right) w^{2} \frac{\phi_{r}}{r^{2}} \delta \phi_{\theta}\right. \\
& +G_{s} w^{2} \frac{d \phi_{\theta}}{d r} \delta\left(\frac{d \phi_{\theta}}{d r}\right)+G_{s} w^{2} \frac{\phi_{r}}{r} \delta\left(\frac{d \phi_{\theta}}{d r}\right)-G_{s} w^{2} \frac{1}{r} \delta \phi_{\theta} \frac{d \phi_{\theta}}{d r} \\
& \left.\left.\left.-G_{s} w^{2} \frac{\phi_{\theta}}{r} \delta\left(\frac{d \phi_{\theta}}{d r}\right)+G_{s}\left(\frac{d w}{d z}\right)^{2} \phi_{\theta} \delta \phi_{\theta}\right\}\right] r d r d z\right\}=0
\end{aligned}
$$

Simplifying further and considering a layered system (Figure 3-1): 


$$
\begin{aligned}
& \left\{\int_{0}^{H_{1}}\left[E_{p} I_{p} \frac{d^{4} w_{1}}{d z^{4}}-2 t_{1} \frac{d^{2} w_{1}}{d z^{2}}+k_{1} w_{1}\right] \delta w_{1} d z+\int_{H_{1}}^{H_{2}}\left[E_{p} I_{p} \frac{d^{4} w_{2}}{d z^{4}}-2 t_{2} \frac{d^{2} w_{2}}{d z^{2}}+k_{2} w_{2}\right] \delta w_{2} d z\right. \\
& +\ldots .+\int_{H_{n-1}}^{L_{p}}\left[E_{p} I_{p} \frac{d^{4} w_{n}}{d z^{4}}-2 t_{i} \frac{d^{2} w_{n}}{d z^{2}}+k_{i} w_{n}\right] \delta w_{n} d z+\int_{L_{p}}^{\infty}\left[-2 t_{n+1} \frac{d^{2} w_{n+1}}{d z^{2}}+k_{n} w_{n+1}\right] \delta w_{n+1} d z \\
& +\left.\left[E_{p} I_{p} \frac{d^{3} w_{1}}{d z^{3}}-2 t_{1} \frac{d w_{1}}{d z}-F_{a}\right] \delta w_{1}\right|_{z=0}-\left.\left[E_{p} I_{p} \frac{d^{2} w_{1}}{d z^{2}}-M_{a}\right] \delta\left(\frac{d w_{1}}{d z}\right)\right|_{z=0} \\
& +\left[-\left.\left(E_{p} I_{p} \frac{d^{3} w_{1}}{d z^{3}}-2 t_{1} \frac{d w_{1}}{d z}\right) \delta w_{1}\right|_{z=H_{1}}+\left.\left(E_{p} I_{p} \frac{d^{3} w_{2}}{d z^{3}}-2 t_{2} \frac{d w_{2}}{d z}\right) \delta w_{2}\right|_{z=H_{1}}\right] \\
& +\left[\left.E_{p} I_{p} \frac{d^{2} w_{1}}{d z^{2}} \delta\left(\frac{d w_{1}}{d z}\right)\right|_{z=H_{1}}-\left.E_{p} I_{p} \frac{d^{2} w_{2}}{d z^{2}} \delta\left(\frac{d w_{2}}{d z}\right)\right|_{z=H_{1}}\right] \\
& \left.+\ldots .+\left.\left[-\left(E_{p} I_{p} \frac{d^{3} w_{n}}{d z^{3}}-2 t_{n} \frac{d w_{n}}{d z}\right\}\right) \delta w_{n}\right|_{z=L_{p}}-\left.2 t_{n+1} \frac{d w_{n+1}}{d z} \delta w_{n+1}\right|_{z=L_{p}}\right] \\
& \left.+\left.E_{p} I_{p} \frac{d^{2} w_{n}}{d z^{2}} \delta\left(\frac{d w_{n}}{d z}\right)\right|_{z=L_{p}}+\left.2 t_{n+1} \frac{d w_{n+1}}{d z} \delta w_{n+1}\right|_{z=\infty}\right\} \\
& +\left\{\int_{r_{p}}^{\infty}\left[-m_{s 1}\left(r \frac{d^{2} \phi_{r}}{d r^{2}}+\frac{d \phi_{r}}{d r}\right)+\left(m_{s 3}+m_{s 2}\right) \frac{d \phi_{\theta}}{d r}+m_{s 4} \frac{\phi_{r}}{r}-m_{s 4} \frac{\phi_{\theta}}{r}+n_{s} r \phi_{r}\right] \delta \phi_{r} d r\right. \\
& \left.+\left.m_{s 1} r \frac{d \phi_{r}}{d r} \delta \phi_{r}\right|_{r_{p}} ^{\infty}+\left.m_{s 3} \phi_{r} \delta \phi_{r}\right|_{r_{p}} ^{\infty}-\left.m_{s 3} \phi_{\theta} \delta \phi_{r}\right|_{r_{p}} ^{\infty}\right\} \\
& +\left\{\int_{r_{p}}^{\infty}\left[-m_{s 2}\left(r \frac{d^{2} \phi_{\theta}}{d r^{2}}+\frac{d \phi_{\theta}}{d r}\right)-\left(m_{s 2}+m_{s 3}\right) \frac{d \phi_{r}}{d r}+m_{s 4} \frac{\phi_{\theta}}{r}-m_{s 4} \frac{\phi_{r}}{r}+n_{s} r \phi_{\theta}\right] \delta \phi_{\theta} d r\right. \\
& \left.+\left.m_{s 2} r \frac{d \phi_{\theta}}{d r} \delta \phi_{\theta}\right|_{r_{p}} ^{\infty}+\left.m_{s 2} \phi_{r} \delta \phi_{\theta}\right|_{r_{p}} ^{\infty}-\left.m_{s 2} \phi_{\theta} \delta \phi_{\theta}\right|_{r_{p}} ^{\infty}\right\}=0
\end{aligned}
$$

where

$$
\begin{aligned}
& t_{i}=\left\{\begin{array}{l}
\frac{\pi}{2} G_{s i} r_{p}^{2}\left(\xi_{1}+\xi_{2}\right) ; i=1,2, \ldots, n \\
\frac{\pi}{2} G_{s n} r_{p}^{2}\left(\xi_{1}+\xi_{2}+1\right) ; i=n+1
\end{array}\right. \\
& \xi_{1}=\frac{1}{r_{p}^{2}} \int_{r_{p}}^{\infty} r \phi_{r}^{2} d r
\end{aligned}
$$




$$
\begin{aligned}
& \xi_{2}=\frac{1}{r_{p}^{2}} \int_{r_{p}}^{\infty} r \phi_{\theta}^{2} d r \\
& k_{i}=\pi\left[\left(\lambda_{s i}+2 G_{s i}\right) \eta_{1}+G_{s i} \eta_{2}+2 \lambda_{s i}\left(\eta_{3}-\eta_{5}\right)-2 G_{s i}\left(\eta_{4}-\eta_{6}\right)\right. \\
& \left.+\left(\lambda_{s i}+3 G_{s i}\right)\left(\eta_{7}+\eta_{8}-2 \eta_{9}\right)\right] \\
& \eta_{1}=\int_{r_{p}}^{\infty} r\left(\frac{d \phi_{r}}{d r}\right)^{2} d r \\
& \eta_{2}=\int_{r_{p}}^{\infty} r\left(\frac{d \phi_{\theta}}{d r}\right)^{2} d r \\
& \eta_{3}=\int_{r_{p}}^{\infty} \phi_{r} \frac{d \phi_{r}}{d r} d r \\
& \eta_{4}=\int_{r_{p}}^{\infty} \phi_{\theta} \frac{d \phi_{\theta}}{d r} d r \\
& \eta_{5}=\int_{r_{p}}^{\infty} \phi_{\theta} \frac{d \phi_{r}}{d r} d r \\
& \eta_{6}=\int_{r_{p}}^{\infty} \phi_{r} \frac{d \phi_{\theta}}{d r} d r \\
& \eta_{7}=\int_{r_{p}}^{\infty} \frac{1}{r} \phi_{r}^{2} d r \\
& \eta_{8}=\int_{r_{p}}^{\infty} \frac{1}{r} \phi_{\theta}^{2} d r \\
& \eta_{9}=\int_{r_{p}}^{\infty} \frac{1}{r} \phi_{r} \phi_{\theta} d r \\
& m_{s 1}=\left(\lambda_{s}+2 G_{s}\right) \int_{0}^{\infty} w^{2} d z=\sum_{i=1}^{n+1}\left(\lambda_{s i}+2 G_{s i}\right) \int_{H_{i-1}}^{H_{i}} w_{i}^{2} d z \\
& m_{s 2}=G_{s} \int_{0}^{\infty} w^{2} d z=\sum_{i=1}^{n+1} G_{s i} \int_{H_{i-1}}^{H_{i}} w_{i}^{2} d z \\
& m_{s 3}=\lambda_{s} \int_{0}^{\infty} w^{2} d z=\sum_{i=1}^{n+1} \lambda_{s i} \int_{H_{i-1}}^{H_{i}} w_{i}^{2} d z
\end{aligned}
$$




$$
\begin{aligned}
& m_{s 4}=\left(\lambda_{s}+3 G_{s}\right) \int_{0}^{\infty} w^{2} d z=\sum_{i=1}^{n+1}\left(\lambda_{s i}+3 G_{s i}\right) \int_{H_{i-1}}^{H_{i}} w_{i}^{2} d z \\
& n_{s}=G_{s} \int_{0}^{\infty}\left(\frac{d w}{d z}\right)^{2} d z=\sum_{i=1}^{n+1} G_{s i} \int_{H_{i-1}}^{H_{i}}\left(\frac{d w_{i}}{d z}\right)^{2} d z
\end{aligned}
$$

The subscript $i$ in the above equations refers to the $i^{\text {th }}$ layer of the multi-layered continuum (Figure 3-1); $w_{i}$ represents the function $w(z)$ in the $i^{\text {th }}$ layer with $\left.w_{i}\right|_{z=H_{i}}=\left.w_{i+1}\right|_{z=H_{i}}$. Note that the $n^{\text {th }}$ (bottom) layer is split into two parts with the part below the pile denoted by the subscript $n+1$; therefore, in the above equations, $H_{n}=L_{p}$ and $H_{n+1} \rightarrow \infty$.

Equation (3-8) is of the form:

$$
\delta \Pi=\left\{A(w) \delta w+B(w) \delta\left(\frac{d w}{d z}\right)\right\}+\left\{C\left(\phi_{r}\right) \delta \phi_{r}\right\}+\left\{D\left(\phi_{\theta}\right) \delta \phi_{\theta}\right\}=0
$$

Since the variations $\delta w(z), \delta(d w / d z), \delta \phi_{r}(r)$ and $\delta \phi_{\theta}(r)$ of the functions $w(z)$ (and its derivative), $\phi_{r}(r)$ and $\phi_{\theta}(r)$ are independent, the terms associated with each of these variations must individually be equal to zero (i.e., $A(w)=0, B(w)=0, C\left(\phi_{r}\right)=0$ and $\left.D\left(\phi_{\theta}\right)=0\right)$ in order to satisfy the condition $\delta \Pi=0$. The resulting equations produce the optimal functions $w_{\mathrm{opt}}(z)$, $\phi_{r, \text { opt }}(r)$ and $\phi_{\theta, \text { opt }}(r)$ that describe the equilibrium configuration of the pile-soil system.

While considering the terms of the variation of potential energy related to $w$, we do so for the following sub-domains: $0 \leq z \leq H_{1}, H_{1} \leq z \leq H_{2}, \ldots, H_{n-1} \leq z \leq L_{p}$, and $L_{p} \leq z<\infty$. Accordingly, $w$ is forced to satisfy equilibrium within each of these sub-domains and hence over the entire domain. For $\phi_{r}$ and $\phi_{\theta}$, the domain over which the potential energy and its variation are calculated is $r_{p} \leq r<\infty$.

\subsubsection{Soil Displacement}

We first consider the variation of $\phi_{r}(r)$. Referring back to equation (3-8) and equation (327), which represents in a simplified manner the form of equation (3-8), we first collect all the terms of $\delta \Pi$ associated with $\delta \phi_{r}$ and add them together, obtaining: 


$$
\begin{aligned}
\int_{r_{p}}^{\infty}[- & \left.-m_{s 1}\left(r \frac{d^{2} \phi_{r}}{d r^{2}}+\frac{d \phi_{r}}{d r}\right)+\left(m_{s 3}+m_{s 2}\right) \frac{d \phi_{\theta}}{d r}+m_{s 4} \frac{\phi_{r}}{r}-m_{s 4} \frac{\phi_{\theta}}{r}+n_{s} r \phi_{r}\right] \delta \phi_{r} d r \\
& +\left.\left(m_{s 1} r \frac{d \phi_{r}}{d r}+m_{s 3} \phi_{r}-m_{s 3} \phi_{\theta}\right) \delta \phi_{r}\right|_{r_{p}} ^{\infty}=0
\end{aligned}
$$

The last term on the left-hand side of equation (3-28) is a multiple of the subtraction of the value of $\delta \phi_{r}$ at $r=r_{p}$ from the value of $\delta \phi_{r}$ at $r=\infty$ and is therefore identically zero for the boundary conditions of our problem $\left(\phi_{r}=0\right.$ at $r=\infty$ and $\phi_{r}=1$ at $\left.r=r_{p}\right)$. This is so because a known (or prescribed) $\phi_{r}$ implies that $\delta \phi_{r}=0$. After this term is made equal to zero, what is left is an equation of form $\left\{C\left(\phi_{r}\right) \delta \phi_{r}\right\}=0$. As discussed earlier, the function $\phi_{r}(r)$ has a non-zero variation (i.e., $\delta \phi_{r} \neq 0$ ) for $r_{p}<r<\infty$ because $\phi_{r}$ is not known a priori in this interval, so $C\left(\phi_{r}\right)=0$, which means the integrand in equation (3-28) must be set to zero, leading to the following differential equation, which, when solved, yields $\phi_{r, \text { opt: }}$ :

$$
\frac{d^{2} \phi_{r}}{d r^{2}}+\frac{1}{r} \frac{d \phi_{r}}{d r}-\left[\left(\frac{\gamma_{1}}{r}\right)^{2}+\left(\frac{\gamma_{2}}{r_{p}}\right)^{2}\right] \phi_{r}=\frac{\gamma_{3}^{2}}{r} \frac{d \phi_{\theta}}{d r}-\left(\frac{\gamma_{1}}{r}\right)^{2} \phi_{\theta}
$$

where the $\gamma$ s are dimensionless constants given by:

$$
\begin{aligned}
& \gamma_{1}^{2}=\frac{m_{s 4}}{m_{s 1}} \\
& \left(\frac{\gamma_{2}}{r_{p}}\right)^{2}=\frac{n_{s}}{m_{s 1}} \\
& \gamma_{3}^{2}=\frac{m_{s 2}+m_{s 3}}{m_{s 1}}
\end{aligned}
$$

We now consider the variation of $\phi_{\phi}(r)$. We collect the terms of equation (3-8) containing $\delta \phi_{\theta}$ and, following a similar procedure as for $\phi_{r}$, we get the following governing differential equation for $\phi_{\theta}$ :

$$
\frac{d^{2} \phi_{\theta}}{d r^{2}}+\frac{1}{r} \frac{d \phi_{\theta}}{d r}-\left[\left(\frac{\gamma_{4}}{r}\right)^{2}+\left(\frac{\gamma_{5}}{r_{p}}\right)^{2}\right] \phi_{\theta}=-\frac{\gamma_{6}^{2}}{r} \frac{d \phi_{r}}{d r}-\left(\frac{\gamma_{4}}{r}\right)^{2} \phi_{r}
$$

with the boundary conditions that $\phi_{\theta}=0$ at $r=\infty$ and $\phi_{\theta}=1$ at $r=r_{p}$, where 


$$
\begin{aligned}
& \gamma_{4}^{2}=\frac{m_{s 4}}{m_{s 2}} \\
& \left(\frac{\gamma_{5}}{r_{p}}\right)^{2}=\frac{n_{s}}{m_{s 2}} \\
& \gamma_{6}^{2}=\frac{m_{s 2}+m_{s 3}}{m_{s 2}}
\end{aligned}
$$

\subsubsection{Pile Deflection}

Finally, we consider the variation of the function $w$ and its derivative. We again refer back to equation (3-8), collect all the terms associated with $\delta w$ and $\delta\left(\frac{d w}{d z}\right)$ and equate their sum to zero:

$$
\begin{aligned}
\int_{0}^{H_{1}} & {\left[E_{p} I_{p} \frac{d^{4} w_{1}}{d z^{4}}-2 t_{1} \frac{d^{2} w_{1}}{d z^{2}}+k_{1} w_{1}\right] \delta w_{1} d z+\int_{H_{1}}^{H_{2}}\left[E_{p} I_{p} \frac{d^{4} w_{2}}{d z^{4}}-2 t_{2} \frac{d^{2} w_{2}}{d z^{2}}+k_{2} w_{2}\right] \delta w_{2} d z } \\
& +\ldots+\int_{H_{n-1}}^{L_{p}}\left[E_{p} I_{p} \frac{d^{4} w_{n}}{d z^{4}}-2 t_{i} \frac{d^{2} w_{n}}{d z^{2}}+k_{i} w_{n}\right] \delta w_{n} d z+\int_{L_{p}}^{\infty}\left[-2 t_{n+1} \frac{d^{2} w_{n+1}}{d z^{2}}+k_{n} w_{n+1}\right] \delta w_{n+1} d z \\
& +\left.\left[E_{p} I_{p} \frac{d^{3} w_{1}}{d z^{3}}-2 t_{1} \frac{d w_{1}}{d z}-F_{a}\right] \delta w_{1}\right|_{z=0}-\left.\left[E_{p} I_{p} \frac{d^{2} w_{1}}{d z^{2}}-M_{a}\right] \delta\left(\frac{d w_{1}}{d z}\right)\right|_{z=0} \\
& +\left[-\left.\left\{E_{p} I_{p} \frac{d^{3} w_{1}}{d z^{3}}-2 t_{1} \frac{d w_{1}}{d z}\right\} \delta w_{1}\right|_{z=H_{1}}+\left.\left\{E_{p} I_{p} \frac{d^{3} w_{2}}{d z^{3}}-2 t_{2} \frac{d w_{2}}{d z}\right\} \delta w_{2}\right|_{z=H_{1}}\right] \\
& +\left[\left.E_{p} I_{p} \frac{d^{2} w_{1}}{d z^{2}} \delta\left(\frac{d w_{1}}{d z}\right)\right|_{z=H_{1}}-\left.E_{p} I_{p} \frac{d^{2} w_{2}}{d z^{2}} \delta\left(\frac{d w_{2}}{d z}\right)\right|_{z=H_{1}}\right] \\
& +\ldots .+\left[-\left.\left\{E_{p} I_{p} \frac{d^{3} w_{n}}{d z^{3}}-2 t_{n} \frac{d w_{n}}{d z}\right\} \delta w_{n}\right|_{z=L_{p}}-\left.2 t_{n+1} \frac{d w_{n+1}}{d z} \delta w_{n+1}\right|_{z=L_{p}}\right] \\
& +\left.E_{p} I_{p} \frac{d^{2} w_{n}}{d z^{2}} \delta\left(\frac{d w_{n}}{d z}\right)\right|_{z=L_{p}}+\left.2 t_{n+1} \frac{d w_{n+1}}{d z} \delta w_{n+1}\right|_{z=\infty}=0
\end{aligned}
$$

We first consider the domain below the pile, i.e., $L_{p} \leq z \leq \infty$. The terms associated with $\delta w$ and $\delta\left(\frac{d w}{d z}\right)$ in equation (37) for $L_{p}<z \leq \infty$ are equated to zero. Since the variation of $w(z)$ with depth is not known a priori within the interior of the domain $L_{p}<z<\infty, \delta w_{n+1} \neq 0$ and so 
the integrand in the integral between $z=L_{p}$ and $z=\infty$ must be equal to zero in order to satisfy equation (3-37). This results in the following differential equation:

$$
2 t_{n+1} \frac{d^{2} w_{n+1}}{d z^{2}}-k_{n} w_{n+1}=0
$$

The displacement in the soil must vanish at infinite vertical distance. We use this as our boundary condition:

$$
w_{n+1}=0(\text { at } z=\infty)
$$

The above equation implies that $\delta w_{n+1}=0$ at $z=\infty$, making the term associated with $\delta w$ at $z=\infty$ equal to zero (which is of course required to satisfy equation (3-37)).

The solution of equation (3-38) satisfying the boundary condition (3-39) is:

$$
w_{n+1}=\left.w_{n}\right|_{z=L_{p}} e^{-\sqrt{\frac{k_{n}}{2 t_{n+1}}}\left(z-L_{p}\right)}
$$

We now consider the function $w$ for the domains $0 \leq z \leq H_{1}, H_{1} \leq z \leq H_{2}, \ldots, H_{n-1} \leq z \leq L_{p}$. The terms containing $\delta w$ and $\delta\left(\frac{d w}{d z}\right)$ in equation (3-37) are equated to zero for each domain. Considering the integrals associated with each individual layer (or each domain $H_{i-1}<z<H_{i}$ ), the integrand for each of these integrals must equal zero because $\delta w_{i}=0$ (as the variation of $w_{i}$ with $z$ within the domains is not known a priori). This gives us the differential equation for the $i^{\text {th }}$ layer, which, expressed in terms of normalized depth $\tilde{z}=z / L_{p}$ and displacement $\tilde{w}=w / L_{p}$ (see equations (2-31a) through (2-31d) for the normalization), is given by:

$$
\frac{d^{4} \tilde{w}_{i}}{d \tilde{z}^{4}}-2 \tilde{t}_{i} \frac{d^{2} \tilde{w}_{i}}{d \tilde{z}^{2}}+\tilde{k}_{i} \tilde{w}_{i}=0
$$

The terms associated with the boundaries of each domain (i.e., at $z=H_{i}$ ) in equation (3-37) must also each be equal to zero. For each layer, there are two terms: one multiplying $\delta w_{i}$ and another multiplying $\delta\left(d w_{i} / d z\right)$. Setting each separately equal to zero yields the boundary conditions for the differential equations represented by equation (3-41). These terms can be seen to be a product of an expression and the variation of the displacement or of its derivative. If the displacement or its derivative is specified at the boundary, then its variation is equal to zero; otherwise, the expression multiplying the variation of the displacement or of its derivative is equal to zero. The boundary conditions at the pile head $(z=\tilde{z}=0)$ are as follows: 


$$
\tilde{w}_{1}=\text { constant }
$$

or

$$
\frac{d^{3} \tilde{w}_{1}}{d \tilde{z}^{3}}-2 \tilde{t}_{1} \frac{d \tilde{w}_{1}}{d \tilde{z}}-\tilde{F}_{a}=0
$$

and

$$
\frac{d \tilde{w}_{1}}{d \tilde{z}}=\text { constant }
$$

or

$$
\frac{d^{2} \widetilde{w}_{1}}{d \widetilde{z}^{2}}-\widetilde{M}_{a}=0
$$

At the interface between any two layers $\left(z=H_{i}\right.$ or $\left.\tilde{z}=\tilde{H}_{i}\right)$ :

$$
\begin{aligned}
& \widetilde{w}_{i}=\widetilde{w}_{i+1} \\
& \frac{d \widetilde{w}_{i}}{d \widetilde{z}}=\frac{d \widetilde{w}_{i+1}}{d \tilde{z}} \\
& \frac{d^{3} \tilde{w}_{i}}{d \tilde{z}^{3}}-2 \tilde{t}_{i} \frac{d \tilde{w}_{i}}{d \tilde{z}}=\frac{d^{3} \tilde{w}_{i+1}}{d \tilde{z}^{3}}-2 \tilde{t}_{i+1} \frac{d \tilde{w}_{i+1}}{d \tilde{z}} \\
& \frac{d^{2} \widetilde{w}_{i}}{d \widetilde{z}^{2}}=\frac{d^{2} \widetilde{w}_{i+1}}{d \widetilde{z}^{2}}
\end{aligned}
$$

At the pile base $\left(z=L_{p}\right.$ or $\left.\tilde{z}=1\right)$, the boundary conditions are:

$$
\tilde{w}_{n}=\text { constant }
$$

or

$$
\begin{aligned}
& \frac{d^{3} \tilde{w}_{n}}{d \tilde{z}^{3}}-2 \tilde{t}_{n} \frac{d \tilde{w}_{n}}{d \tilde{z}}=-2 \tilde{t}_{n+1} \frac{d \tilde{w}_{n+1}}{d \tilde{z}} \\
& \frac{d \tilde{w}_{n}}{d z}=\text { constant }
\end{aligned}
$$

or

$$
\frac{d^{2} \tilde{w}_{n}}{d \tilde{z}^{2}}=0
$$

Equation (3-44b) is further simplified and solely expressed in terms of $\tilde{w}_{n}$ by differentiating $w_{n+1}$ in equation (3-40) with respect to $z$, normalizing the expression and then substituting it back into equation (3-44b) to yield: 


$$
\frac{d^{3} \tilde{w}_{n}}{d \tilde{z}^{3}}-2 \tilde{t}_{n} \frac{d \tilde{w}_{n}}{d \tilde{z}}-\sqrt{2 \tilde{k}_{n} \tilde{t}_{n+1}} \tilde{w}_{n}=0
$$

The dimensionless terms in the above equations are defined as follows:

$$
\begin{aligned}
& \tilde{t}_{i}=\frac{t_{i} L_{p}^{2}}{E_{p} I_{p}} \\
& \tilde{k}_{i}=\frac{k_{i} L_{p}^{4}}{E_{p} I_{p}} \\
& \tilde{F}_{a}=\frac{F_{a} L_{p}^{2}}{E_{p} I_{p}} \\
& \tilde{M}_{a}=\frac{M_{a} L_{p}}{E_{p} I_{p}} \\
& \tilde{H}_{i}=\frac{H_{i}}{L_{p}}
\end{aligned}
$$

The similarity between the above equations of pile deflection with those of chapter 2 must be apparent. Using the boundary conditions, we satisfy the equilibrium and continuity of forces and displacements, respectively, over the entire pile length. The right hand side of equation (3-44) represents the shear force just below the pile base (note that the right hand side does not contain any shear from the pile section because below the pile base the contribution can come only from the soil). This base shear acts only if the pile base displaces horizontally (i.e., if the base is floating, which results in nonzero $\left.w_{n+1}\right)$. Analytical solution of the pile equation is obtained using MIP described in chapter 2.

\subsubsection{Expression of $\gamma$ 's in Terms of Dimensionless Deflections}

The $\gamma$ 's in equations (3-30) though (3-32) and (3-34) through (3-36) are expressed in terms of the dimensionless pile deflection and slope as follows:

$$
\gamma_{1}=\sqrt{\frac{\sum_{i=1}^{n}\left(\lambda_{s i}+3 G_{s i}\right) \int_{\tilde{H}_{i-1}}^{\tilde{H}_{i}} \tilde{w}_{i}^{2} d \tilde{z}+\left.\left(\lambda_{s n}+3 G_{s n}\right) \sqrt{\frac{\tilde{t}_{n+1}}{2 \tilde{k}_{n}}} \tilde{w}_{n}^{2}\right|_{\tilde{z}=1}}{\sum_{i=1}^{n}\left(\lambda_{s i}+2 G_{s i}\right) \int_{\tilde{H}_{i-1}}^{\tilde{H}_{i}} \tilde{w}_{i}^{2} d \tilde{z}+\left.\left(\lambda_{s n}+2 G_{s n}\right) \sqrt{\frac{\tilde{t}_{n+1}}{2 \tilde{k}_{n}}} \tilde{w}_{n}^{2}\right|_{\tilde{z}=1}}}
$$


$\gamma_{2}=\sqrt{\frac{1}{\psi^{2}} \frac{\sum_{i=1}^{n} G_{s i} \int_{\tilde{H}_{i-1}}^{\tilde{H}_{i}}\left(\frac{d \tilde{w}_{i}}{d \tilde{z}}\right)^{2} d \tilde{z}+\left.G_{s n} \sqrt{\frac{\tilde{k}_{n}}{8 \tilde{t}_{n+1}}} \tilde{w}_{n}^{2}\right|_{\tilde{z}=1}\left(\lambda_{s i}+2 G_{s i}\right) \int_{\tilde{H}_{i-1}}^{\tilde{H}_{i}} \tilde{w}_{i}^{2} d \tilde{z}+\left(\lambda_{s n}+2 G_{s n}\right) \sqrt{\left.\frac{\tilde{t}_{n+1}}{2 \tilde{k}_{n}} \tilde{w}_{n}^{2}\right|_{\tilde{z}=1}}}{}}$

$\gamma_{3}=\sqrt{\frac{\sum_{i=1}^{n}\left(\lambda_{s i}+G_{s i}\right) \int_{\tilde{H}_{i-1}}^{\tilde{H}_{i}} \tilde{w}_{i}^{2} d \tilde{z}+\left.\left(\lambda_{s n}+G_{s n}\right) \sqrt{\frac{\tilde{t}_{n+1}}{2 \tilde{k}_{n}}} \tilde{w}_{n}^{2}\right|_{\tilde{z}=1}}{\sum_{i=1}^{n}\left(\lambda_{s i}+2 G_{s i}\right) \int_{\tilde{H}_{i-1}}^{\tilde{H}_{i}} \tilde{w}_{i}^{2} d \tilde{z}+\left.\left(\lambda_{s n}+2 G_{s n}\right) \sqrt{\frac{\tilde{t}_{n+1}}{2 \tilde{k}_{n}}} \tilde{w}_{n}^{2}\right|_{\tilde{z}=1}}}$

$\gamma_{4}=\sqrt{\frac{\sum_{i=1}^{n}\left(\lambda_{s i}+3 G_{s i}\right) \int_{\tilde{H}_{i-1}}^{\tilde{H}_{i}} \tilde{w}_{i}^{2} d \tilde{z}+\left.\left(\lambda_{s n}+3 G_{s n}\right) \sqrt{\frac{\tilde{t}_{n+1}}{2 \tilde{k}_{n}}} \tilde{w}_{n}^{2}\right|_{\tilde{z}=1}}{\sum_{i=1}^{n} G_{s i} \int_{\tilde{H}_{i-1}}^{\tilde{H}_{i}} \tilde{w}_{i}^{2} d \tilde{z}+\left.G_{s n} \sqrt{\frac{\tilde{t}_{n+1}}{2 \tilde{k}_{n}}} \tilde{w}_{n}^{2}\right|_{\tilde{z}=1}}}$

$\gamma_{5}=\sqrt{\frac{1}{\psi^{2}} \frac{\sum_{i=1}^{n} G_{s i} \int_{\tilde{H}_{i-1}}^{\tilde{H}_{i}}\left(\frac{d \tilde{w}_{i}}{d \tilde{z}}\right)^{2} d \tilde{z}+\left.G_{s n} \sqrt{\frac{\tilde{k}_{n}}{8 \tilde{t}_{n+1}}} \tilde{w}_{n}^{2}\right|_{\tilde{z}=1}}{\sum_{i=1}^{n} G_{s i} \int_{\tilde{H}_{i-1}}^{\tilde{H}_{i}} \tilde{w}_{i}^{2} d \tilde{z}+G_{s n} \sqrt{\left.\frac{\tilde{t}_{n+1}}{2 \tilde{k}_{n}} \tilde{w}_{n}^{2}\right|_{\tilde{z}=1}}}}$

$\gamma_{6}=\sqrt{\frac{\sum_{i=1}^{n}\left(\lambda_{s i}+G_{s i}\right) \int_{\tilde{H}_{i-1}}^{\tilde{H}_{i}} \tilde{w}_{i}^{2} d \tilde{z}+\left(\lambda_{s n}+G_{s n}\right) \sqrt{\frac{\tilde{t}_{n+1}}{2 \tilde{k}_{n}}}}{\sum_{i=1}^{n} G_{s i} \int_{\tilde{H}_{i-1}}^{2} \tilde{w}_{i}^{2} d \tilde{z}+\left.G_{s n} \sqrt{\frac{\tilde{t}_{n+1}}{2 \tilde{k}_{n}}} \tilde{w}_{n}^{2}\right|_{\tilde{z}=1}}}$

where $\psi=L_{p} / r_{p}$. These expressions can be directly used in the computations.

\subsection{Finite Difference Solution for Soil Displacements}

The differential equations (3-29) and (3-33) for $\phi_{r}$ and $\phi_{\theta}$ are solved using the finite difference method. The equations are interdependent, and hence, must be solved simultaneously. Using the central-difference scheme, equations (3-29) and (3-33) can be respectively written as: 


$$
\begin{aligned}
& \frac{\phi_{r}^{j+1}-2 \phi_{r}^{j}+\phi_{r}^{j-1}}{\Delta r^{2}}+\frac{1}{r_{j}} \frac{\phi_{r}^{j+1}-\phi_{r}^{j-1}}{2 \Delta r}-\left[\left(\frac{\gamma_{1}}{r_{j}}\right)^{2}+\left(\frac{\gamma_{2}}{r_{p}}\right)^{2}\right] \phi_{r}^{j}=\frac{\gamma_{3}^{2}}{r_{j}} \frac{\phi_{\theta}^{j+1}-\phi_{\theta}^{j-1}}{2 \Delta r}-\left(\frac{\gamma_{1}}{r_{j}}\right)^{2} \phi_{\theta}^{j} \\
& \frac{\phi_{\theta}^{j+1}-2 \phi_{\theta}^{j}+\phi_{\theta}^{j-1}}{\Delta r^{2}}+\frac{1}{r_{j}} \frac{\phi_{\theta}^{j+1}-\phi_{\theta}^{j-1}}{2 \Delta r}-\left[\left(\frac{\gamma_{4}}{r_{j}}\right)^{2}+\left(\frac{\gamma_{5}}{r_{p}}\right)^{2}\right] \phi_{\theta}^{j}=-\frac{\gamma_{6}^{2}}{r_{j}} \frac{\phi_{r}^{j+1}-\phi_{r}^{j-1}}{2 \Delta r}-\left(\frac{\gamma_{4}}{r_{j}}\right)^{2} \phi_{r}^{j}
\end{aligned}
$$

where $j$ represents the $j^{\text {th }}$ node, which is at a radial distance $r_{j}$ from the pile axis; and $\Delta r$ is the distance between consecutive nodes (discretization length). The total number of discretized nodes $m$ should be sufficiently large so that the infinite domain in the radial direction can be adequately modeled (Figure 3-4). The discretization length $\Delta r$ should be sufficiently small to maintain a satisfactory level of accuracy.

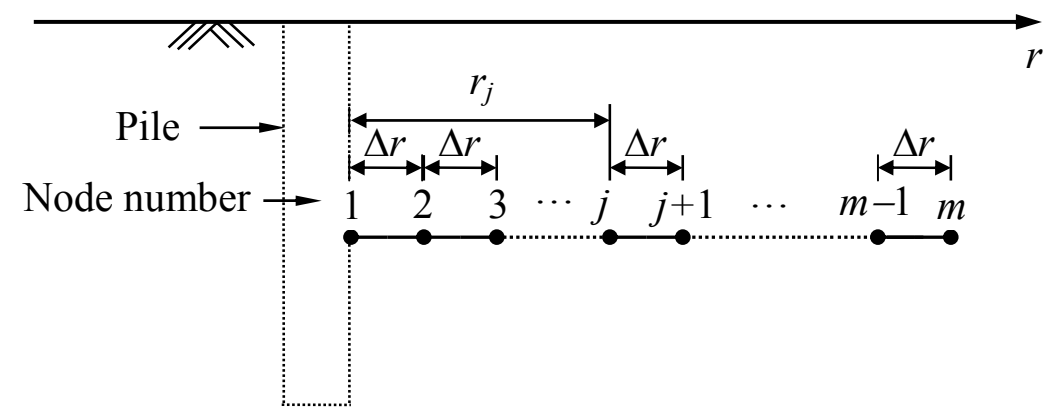

Figure 3-4 Finite difference discretization for $\phi_{r}$ and $\phi_{\theta}$

Equation (3-57) along with the boundary conditions $\phi_{r}^{(1)}=1$ (at $r=r_{p}$ ) and $\phi_{r}{ }^{(m)}=0$ (at $r$ $=\infty$ ) is applied to the discretized nodes, which yields the following equation: 


$$
\begin{aligned}
& {\left[\begin{array}{cccccccccc}
1 & 0 & 0 & \ldots & & & & & \cdots & 0 \\
0 & K_{2,2}^{\phi_{r}} & K_{2,3}^{\phi_{r}} & 0 & \ldots & & & & \ldots & 0 \\
0 & K_{3,2}^{\phi_{r}} & K_{3,3}^{\phi_{r}} & K_{3,4}^{\phi_{r}} & 0 & \ldots & & & \ldots & 0 \\
0 & 0 & K_{4,3}^{\phi_{r}} & K_{4,4}^{\phi_{r}} & K_{4,5}^{\phi_{r}} & 0 & \ldots & & \ldots & 0 \\
\vdots & & \ddots & \ddots & \ddots & \ddots & \ddots & & \ldots & \vdots \\
0 & \ldots & \ldots & 0 & K_{j, j-1}^{\phi_{r}} & K_{j, j}^{\phi_{r}} & K_{j, j+1}^{\phi_{r}} & 0 & \ldots & 0 \\
\vdots & & & & \ddots & \ddots & \ddots & \ddots & \ddots & \vdots \\
0 & \cdots & & & \ldots & 0 & K_{m-3, m-2}^{\phi_{r}} & K_{m-2, m-2}^{\phi_{r}} & K_{m-2, m-1}^{\phi_{r}} & 0 \\
0 & \cdots & & & & \ldots & 0 & K_{m-1, m-2}^{\phi_{r}} & K_{m-1, m-1}^{\phi_{r}} & 0 \\
0 & \cdots & & & & & \ldots & 0 & 0 & 1
\end{array}\right]\left[\begin{array}{c}
\phi_{r}^{(1)} \\
\phi_{r}^{(2)} \\
\phi_{r}^{(3)} \\
\phi_{r}^{(4)} \\
\vdots \\
\phi_{r}^{(j)} \\
\vdots \\
\phi_{r}^{(m-2)} \\
\phi_{r}^{(m-1)} \\
\phi_{r}^{(m)}
\end{array}\right]} \\
& =\left[\begin{array}{c}
1 \\
F_{2}^{\phi_{r}} \\
F_{3}^{\phi_{r}} \\
F_{4}^{\phi_{r}} \\
\vdots \\
F_{j}^{\phi_{r}} \\
\vdots \\
F_{m-2}^{\phi_{r}} \\
F_{m-1}^{\phi_{r}} \\
0
\end{array}\right]
\end{aligned}
$$

The nonzero elements of the left-hand side matrix $\left[K^{\phi_{r}}\right]_{m \times m}$ in the above equation are given by:

$$
\begin{aligned}
& K_{j, j-1}^{\phi_{r}}=\frac{1}{\Delta r^{2}}-\frac{1}{2 r_{j} \Delta r} \\
& K_{j, j}^{\phi_{r}}=-\frac{2}{\Delta r^{2}}-\left[\left(\frac{\gamma_{1}}{r_{j}}\right)^{2}+\left(\frac{\gamma_{2}}{r_{p}}\right)^{2}\right] \\
& K_{j, j+1}^{\phi_{r}}=\frac{1}{\Delta r^{2}}+\frac{1}{2 r_{j} \Delta r}
\end{aligned}
$$

in which the subscript $j$ is valid for nodes 2 through $m-1$ with the exception that $K_{2,1}^{\phi_{r}}=0$ and $K_{m-1, m}^{\phi_{r}}=0$ (as is evident from equation (3-59)).

The elements of the right-hand side vector $\left\{F^{\phi_{r}}\right\}_{m \times 1}$ in equation (3-59) are given by: 


$$
F_{j}^{\phi_{r}}=\frac{\gamma_{3}^{2}}{r_{j}} \frac{\phi_{\theta}^{j+1}-\phi_{\theta}^{j-1}}{2 \Delta r}-\left(\frac{\gamma_{1}}{r_{j}}\right)^{2} \phi_{\theta}^{j}
$$

where $j$ represents nodes 3 through $m-2$. The elements corresponding to node 2 and $m-1$ are given by:

$$
\begin{aligned}
& F_{2}^{\phi_{r}}=-\frac{1}{\Delta r^{2}}+\frac{1}{2 r_{2} \Delta r}+\frac{\gamma_{3}^{2}}{r_{2}} \frac{\phi_{\theta}^{(3)}-1}{2 \Delta r}-\left(\frac{\gamma_{1}}{r_{2}}\right)^{2} \phi_{\theta}^{(2)} \\
& F_{m-1}^{\phi_{r}}=\frac{\gamma_{3}^{2}}{r_{m-1}} \frac{-\phi_{\theta}^{m-2}}{2 \Delta r}-\left(\frac{\gamma_{1}}{r_{m-1}}\right)^{2} \phi_{\theta}^{m-1}
\end{aligned}
$$

Using equation (3-58) and the boundary conditions that $\phi_{\theta}^{(1)}=1$ (at $r=r_{p}$ ) and $\phi_{\theta}{ }^{(m)}=0$ (at $r=\infty$ ), a matrix equation (similar to equation (3-59)) for $\phi_{\theta}$ can also be formed for the discretized nodes:

$$
\left[K^{\phi_{\theta}}\right]\left\{\phi_{\theta}\right\}=\left\{F^{\phi_{\theta}}\right\}
$$

The number and positioning of the nonzero elements of $\left[K^{\phi_{\theta}}\right]_{m \times m}$ in equation (3-66) are exactly the same as that of $\left[K^{\phi_{r}}\right]_{m \times m}$ of equation (3-59). The expressions of the off-diagonal elements of $\left[K^{\phi_{\theta}}\right]$ and $\left[K^{\phi_{r}}\right]_{m \times m}$ are also the same (i.e., $K_{p, q}^{\phi_{r}}=K_{p, q}^{\phi_{\theta}}$ for $p \neq q$ ). The diagonal elements of $\left[K^{\phi_{\theta}}\right]$ for $j=2$ through $m-1$ are given by:

$$
K_{j, j}^{\phi_{r}}=-\frac{2}{\Delta r^{2}}-\left[\left(\frac{\gamma_{4}}{r_{j}}\right)^{2}+\left(\frac{\gamma_{5}}{r_{p}}\right)^{2}\right]
$$

The structure of $\left\{F^{\phi_{\theta}}\right\}_{m \times 1}$ in equation (3-66) is also similar to $\left\{F^{\phi_{r}}\right\}_{m \times 1}$ of equation (59) with $F_{1}^{\phi_{\theta}}=1$ and $F_{m}^{\phi_{\theta}}=0$. The remaining elements of $\left\{F^{\phi_{\theta}}\right\}$ are given by:

$$
\begin{aligned}
& F_{j}^{\phi_{\theta}}=-\frac{\gamma_{6}^{2}}{r_{j}} \frac{\phi_{r}^{j+1}-\phi_{r}^{j-1}}{2 \Delta r}-\left(\frac{\gamma_{4}}{r_{j}}\right)^{2} \phi_{r}^{j} \quad(j=3,4, \ldots, m-2) \\
& F_{2}^{\phi_{\theta}}=-\frac{1}{\Delta r^{2}}+\frac{1}{2 r_{2} \Delta r}-\frac{\gamma_{6}^{2}}{r_{2}} \frac{\phi_{r}^{(3)}-1}{2 \Delta r}-\left(\frac{\gamma_{4}}{r_{2}}\right)^{2} \phi_{r}^{(2)}
\end{aligned}
$$




$$
F_{m-1}^{\phi_{\theta}}=-\frac{\gamma_{6}^{2}}{r_{m-1}} \frac{-\phi_{r}^{m-2}}{2 \Delta r}-\left(\frac{\gamma_{4}}{r_{m-1}}\right)^{2} \phi_{r}^{m-1}
$$

Since the right-hand side vectors $\left\{F^{\phi_{r}}\right\}$ and $\left\{F^{\phi_{\theta}}\right\}$ contain the unknowns $\phi_{\theta}$ and $\phi_{r}$, iterations are necessary to obtain their values. An initial estimate of $\phi_{r}^{j}$ is made and given as input to $\left\{F^{\phi_{\theta}}\right\}$, and $\phi_{\theta}^{j}$ is determined by solving equation (3-66). The $\phi_{\theta}^{j}$ values are then given as input to $\left\{F^{\phi_{r}}\right\}$ to obtain $\phi_{r}^{j}$ from equation (3-59). The newly obtained values of $\phi_{r}^{j}$ are again used to obtain new values of $\phi_{\theta}^{j}$, and the iterations are continued until convergence is reached. The criteria $\frac{1}{m} \sum_{j=1}^{m}\left|\phi_{r}^{j_{\text {previous }}}-\phi_{r}^{j_{\text {current }}}\right| \leq 10^{-6}$ and $\frac{1}{m} \sum_{j=1}^{m}\left|\phi_{\theta}^{j_{\text {previous }}}-\phi_{\theta}^{j_{\text {current }}}\right| \leq 10^{-6}$ are used (a stringent value of $10^{-6}$ is used because this iterative solution scheme is central to another set of iterations described next) to ensure that accurate values of $\phi_{r}$ and $\phi_{\theta}$ are obtained.

\subsection{Solution Algorithm}

In order to obtain pile deflections by solving equation (3-41), $k_{i}$ and $t_{i}$ must be known. However, the soil parameters depend on $\phi_{r}$ and $\phi_{\theta}$, which are not known a priori. Hence, an iterative algorithm (separate from the iterations between $\phi_{r}$ and $\phi_{\theta}$ described in the previous section) is necessary to solve the problem. First, initial guesses for $\gamma_{1}$ through $\gamma_{6}$ are made, and for these assumed values, $\phi_{r}$ and $\phi_{\theta}$ are determined using the iterative technique described in the previous section. Using the calculated values of $\phi_{r}$ and $\phi_{\theta}, \eta_{1}$ through $\eta_{9}$ and $\xi_{1}$ and $\xi_{2}$ are calculated by numerical integrations (with $\Delta r$ as the step length) and, subsequently, $k_{i}$ and $t_{i}$ are determined. Using the values of $k_{i}$ and $t_{i}$, the pile deflection is calculated. From the calculated values of pile deflection and slope of the deformed pile, $\gamma_{1}$ through $\gamma_{6}$ are obtained. The new values of $\gamma_{1}$ through $\gamma_{6}$ are then used to recalculate $\phi_{r}$ and $\phi_{\theta}$, and so on. The entire process is repeated until convergence on each of the $\gamma$ s is attained. The tolerance limit prescribed on the $\gamma$ 's between the $i^{\text {th }}$ and $(i+1)^{\text {th }}$ iteration is $\left|\gamma_{1 / 2 / \ldots / 6}^{(i+1)}-\gamma_{1 / 2 / \ldots / 6}^{(i)}\right|<0.001$. The details of the solution steps are given in the form of a flow chart in Figure 3-5. We chose an initial guess of "one" for 
all the $\gamma$ 's but any other choice would produce results with the same level of accuracy at approximately the same computation time.

\subsection{Results}

In this section, we choose a few example problems to investigate if the analysis produces reliable results. We consider as an illustration of use of the analysis a 15-m-long drilled shaft, with a diameter of $0.6 \mathrm{~m}$ and pile modulus $E_{p}=24 \times 10^{6} \mathrm{kN} / \mathrm{m}^{2}$, embedded in a four-layer soil deposit with $E_{s 1}=20 \mathrm{MPa}, E_{s 2}=35 \mathrm{MPa}, E_{s 3}=50 \mathrm{MPa}$ and $E_{s 3}=80 \mathrm{MPa} ; v_{s 1}=0.35, v_{s 2}=0.25$, $v_{s 3}=0.2$ and $v_{s 4}=0.15\left(E_{s i}\right.$ and $v_{s i}$ are the soil Young's modulus and Poisson's ratio for the $i^{\text {th }}$ layer; $E_{s i}$ and $v_{s i}$ are related to $\lambda_{s i}$ and $G_{s i}$ by $\lambda_{s i}=E_{s i} v_{s i} /\left(1+v_{s i}\right)\left(1-2 v_{s i}\right)$ and $\left.G_{s i}=E_{s i} / 2\left(1+v_{s i}\right)\right)$. A horizontal force $F_{a}=300 \mathrm{kN}$ acts on the pile. Figure 3-6 shows the pile deflection profile obtained using our analysis, the analysis based on the displacement assumption of Sun (1994) and a three-dimensional (3D) finite element analysis (FEA). The pile response obtained from our analysis closely matches that of 3D FEA; the analysis based on the displacement field assumed by Sun (1994a) produces a stiffer pile response.

Next, we consider a large-diameter drilled shaft, 40-m long, with a diameter of $1.7 \mathrm{~m}$ and $E_{p}=25 \times 10^{6} \mathrm{kPa}$, embedded in a four-layer soil profile with $H_{1}=1.5 \mathrm{~m}, H_{2}=3.5 \mathrm{~m}$, and $H_{3}=$ $8.5 \mathrm{~m} ; E_{s 1}=20 \mathrm{MPa}, E_{s 2}=25 \mathrm{MPa}, E_{s 3}=40 \mathrm{MPa}$ and $E_{s 4}=80 \mathrm{MPa} ; v_{s 1}=0.35, v_{s 2}=0.3, v_{s 3}=$ 0.25 and $v_{s 4}=0.2$. A $3000 \mathrm{kN}$ force acts at the pile head. Figure 3-7 shows the pile deflection profiles, as obtained from our analysis, the analysis based on the displacement assumption of Sun (1994a) and 3D FEA. As before, our results match those of FEA more closely than results based on the Sun (1994a) assumption. It is important to note that our analysis takes less than a minute to produce the results as opposed to the 3D FEA which takes approximately twenty to thirty minutes to run. 


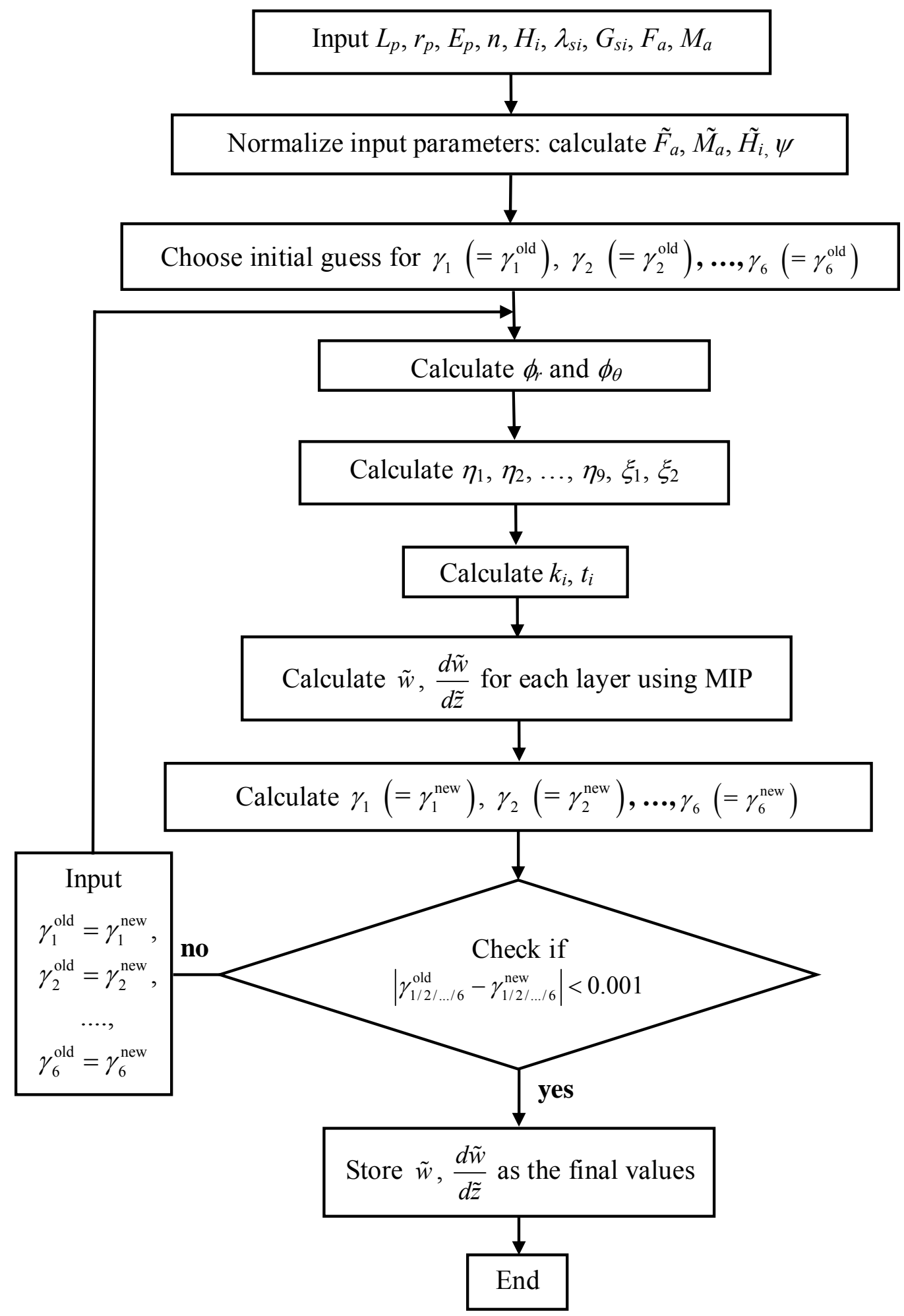

Figure 3-5 Solution Flow Chart 


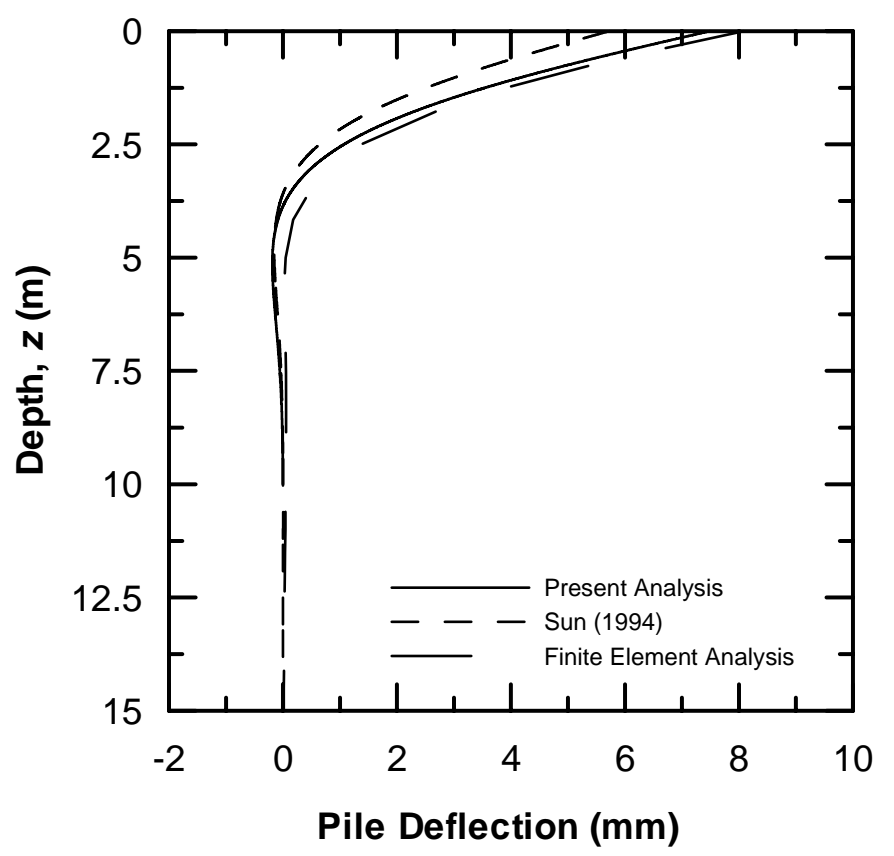

Figure 3-6 Deflection profile of a 15-m-long pile

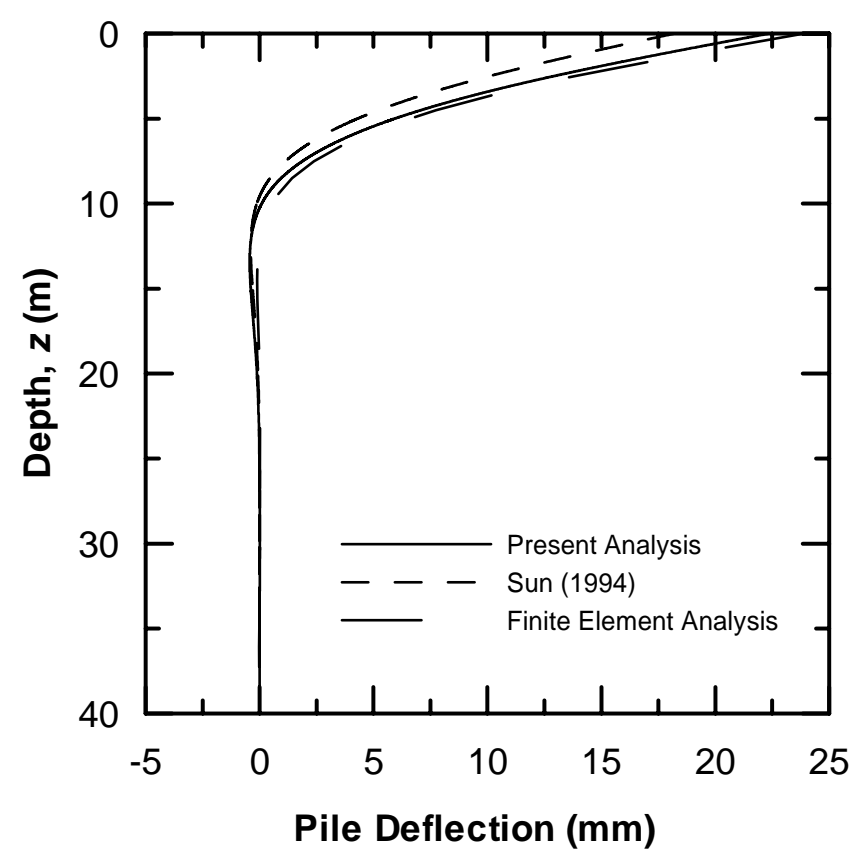

Figure 3-7 Deflection profile of a 40-m-long drilled shaft

Finally, we consider the field example of a laterally loaded pile load test performed by McClelland and Focht (1958). The length $\left(L_{p}\right)$ and radius $\left(r_{p}\right)$ of the pile are $23 \mathrm{~m}$ and $0.305 \mathrm{~m}$, 
and the pile was embedded into a normally consolidated clay. The pile was acted upon by a lateral force $F_{a}=300 \mathrm{kN}$ and a negative moment $M_{a}=-265 \mathrm{kNm}$ at the head. Randolph (1981) back-calculated the pile modulus $E_{p}$ as $68.42 \times 10^{6} \mathrm{kN} / \mathrm{m}^{2}$ from the reported pile flexural rigidity. Randolph (1981) further suggested, based on back calculation of test results to match his finite element analysis (coupled with Fourier series), that the soil shear modulus profile for this soil deposit can be represented as:

$$
G_{s}=0.8 z \times 10^{3} \mathrm{kN} / \mathrm{m}^{2}
$$

with $v_{s}=0.3$. We divided the soil profile into four layers and calculated the shear and Young's moduli at the middle of each layer, which were considered the representative values for each layer (Table 3-1). Using these values of soil modulus, we calculated the pile deflection profile using both our analysis and that based on the assumption of Sun (1994a). Figure 3-8 shows the pile responses. Also plotted are the measured pile response and that obtained by Randolph (1981). Our analysis produces a pile deflection profile that closely matches the measured profile; the analysis of Sun (1994a) produces a stiffer response.

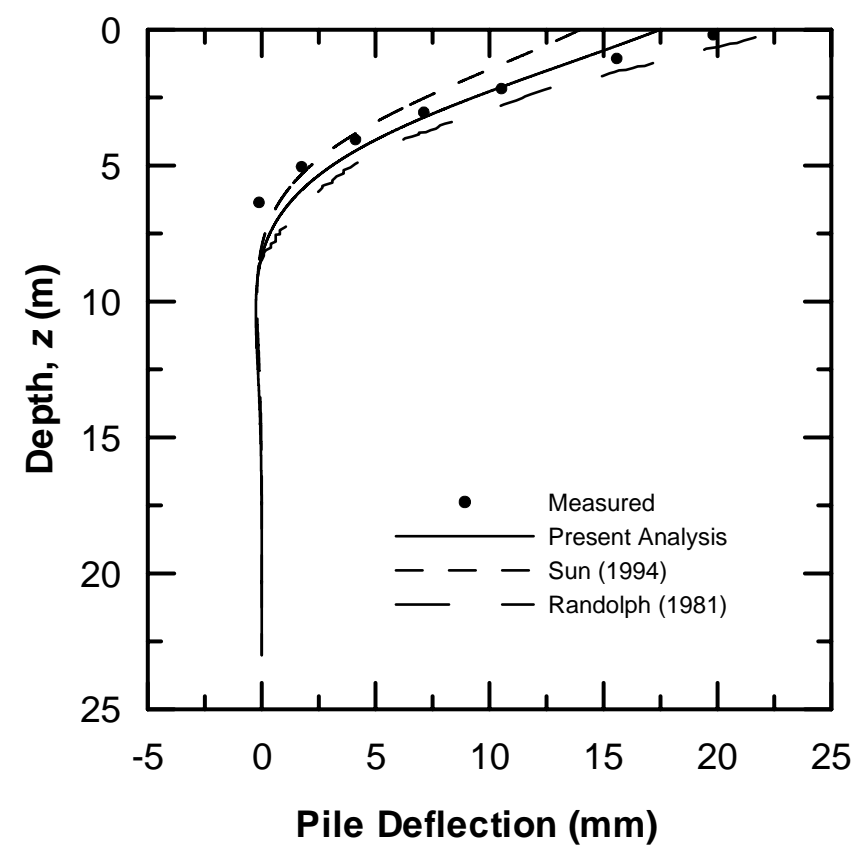

Figure 3-8 Deflection profile for the pile load test of McClelland and Focht (1958) 
Table 3-1 Soil Properties at the Pile Load Test Site of Ismael and Klym (1978)

\begin{tabular}{|c|c|c|c|}
\hline $\begin{array}{c}\text { Depth } \\
\text { (m) }\end{array}$ & $\begin{array}{c}\text { Extent of Soil } \\
\text { Layers (m) }\end{array}$ & $\begin{array}{c}\text { Shear Modulus } \\
G_{s}(\mathrm{MPa})\end{array}$ & $\begin{array}{c}E_{s} \\
\text { (MPa) }\end{array}$ \\
\hline 2.0 & 0 to -4.0 & 1.6 & 4.2 \\
\hline 6.0 & -4.0 to -8.0 & 4.8 & 12.5 \\
\hline 10.0 & -8.0 to -12.0 & 8.0 & 20.8 \\
\hline 17.5 & -12.0 to great depth & 14.0 & 36.4 \\
\hline
\end{tabular}

\subsection{Summary}

A continuum-based elastic analysis for a single, circular pile embedded in a multi-layered elastic medium and subjected to a horizontal force and a moment at the head was developed. The solution is fast and produces results comparable to three-dimensional finite element analysis. Using this method, pile deflection, slope of the deflection curve, bending moment and shear force for the entire length of the pile can be obtained if the following are known: the pile radius and length, thicknesses of the soil layers, Young's modulus of the pile material, the elastic constants of the soil in the various layers, and the magnitudes of the applied force and moment.

The governing differential equation for pile deflection is obtained using the principle of minimum potential energy, and closed-form solutions are obtained. The solution depends on a set of parameters $\gamma_{1}$ through $\gamma_{6}$ that determine the rate at which the deflections in the soil medium decay with increasing radial distance from the pile axis. These parameters are not known a priori and must be determined iteratively. Hence, an iterative scheme was developed to obtain solutions for a variety of boundary conditions and soil profiles consisting of one, two, three and four layers. Notwithstanding the iterations on the $\gamma$ 's, the solutions are obtained in seconds. 


\section{CHAPTER 4. NONLINEAR ANALYSIS OF LATERALLY LOADED PILE IN LAYERED SOIL MEDIUM}

\subsection{Introduction}

In this chapter, we modify the model developed in chapter 3 to account for the nonlinear stress-strain behavior of soil. The degradation (i.e., the decrease in magnitude) of modulus with strain renders the soil mass within each layer heterogeneous because the strains, due to the application of lateral load, are different at different points within the soil mass. Consequently, the equations are modified to take into account the heterogeneity, following which a nonlinear stress-strain relationship is plugged in to develop a nonlinear solution algorithm. The analysis takes into account the three-dimensional pile-soil interaction but produces results much quicker than a standard three-dimensional finite element analysis.

\subsection{Overview}

Scott (1981) had shown with the example of a field pile load test that the head deflection versus load curves for laterally loaded piles are nonlinear, in fact, much more nonlinear than the load-deflection curves for axially-loaded piles. Such a strongly nonlinear response is caused by the nonlinear stress-strain behavior of soil. The resistance against lateral pile movement is generated primarily in the top few meters of soil where the soil is more deformable and weak, which results in a nonlinear pile-soil response. Consequently, it is important to take into account soil nonlinearity in the analysis of laterally loaded piles.

There are two distinct components in the nonlinear analysis of laterally loaded piles: first, studying the soil nonlinearity in the elemental scale and, second, translating the effect of soil nonlinearity from the elemental scale to the scale of the problem so that the overall resistance of soil against pile movement can be properly estimated. Unfortunately, the p-y method is not based on such a rational framework. In the $\mathrm{p}-\mathrm{y}$ method, the nonlinear resistance of soil $p$ is estimated by empirical equations, which are obtained by back fitting the results of numerical 
analysis or field-scale, pile load-test results to match the results of the one-parameter, beam-onfoundation analysis (see chapter 2) by giving different inputs for $p$. Thus, in the p-y method, there is no rational integration of the individual resistance of soil elements to obtain the total soil resistance. The elemental stress-strain properties of soil can be estimated with much more certainty (from laboratory tests, in-situ tests, well established correlations) than the total soil resistance $p$. But the brute-force approach involved in the p-y method often does not allow the reflection of the actual stress-strain behavior and, unless site specific p-y curves are developed from field load tests, there is no guarantee predictions using the p-y method will be accurate.

The limitations of the p-y method can be overcome by a three-dimensional finite element analysis; but the computation expense for such an analysis is prohibitive for its use in routine practice. On the other hand, the analysis framework developed in the previous chapters provides us with an opportunity to extend the linear elastic approach to account for soil nonlinearity.

In this chapter, we rigorously connect the nonlinear stress-strain response of soil to the overall soil resistance against pile movement. We develop the differential equations governing pile deflection and soil displacement, which are similar to those in chapter 3 but have the provisions to incorporate nonlinear stress-strain response. Using these equations, we connect the soil parameters $k_{i}$ and $t_{i}$ (they produce the overall soil resistance) to the soil stress-strain relationships (i.e., the relationships showing the change in the elastic constants $\lambda_{s}$ and $G_{s}$ with strain). Subsequently, we develop a nonlinear solution algorithm which produces the pile response.

\subsection{Soil Nonlinearity}

Soil is a unique material, showing nonlinearity at a very early stage of loading (typical stress-strain plots of soil are shown in Figure 4-1) (Salgado 2008). A typical shear modulus versus shear strain plot makes this point clearer (Figure 4-2). Soil behaves as a linear elastic material at an extremely low range of strain, and the modulus starts degrading at a strain as low as $10^{-5}$ (Shibuya et al. 1992, Salgado 2008). Such a nonlinear behavior can be attributed to the granular nature of the material. If a small load is applied to a soil mass, the soil particles themselves deform but do not move relative to each other, causing very small amount of strain. At this stage, if the load is removed, the stresses acting on the particles are released and no 
permanent deformation is produced. However, if the load is increased further, the soil particles start sliding and rolling over each other, which leads to permanent macroscopic deformation because if the load is removed, the particles can no longer go back to their original positions. Thus, soil deformation, in general, is largely plastic although some elastic fraction is also present. With further increase in load (at very high stress) there can be particle crushing and breakage leading to further plastic deformation. Since, the soil particles constantly change their position during the application of a load, the resistance offered by the soil mass against deformation also changes; this results in the change in the value of soil modulus with increase in strain.

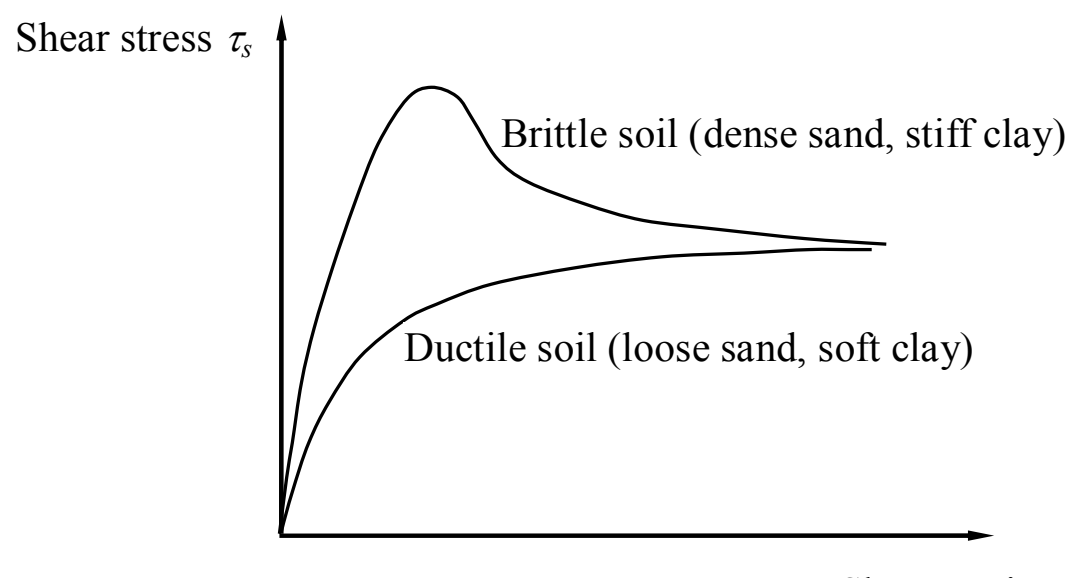

Shear strain $\gamma_{s}$

Figure 4-1 Typical Stress-Strain Plot of Soil under Drained Condition

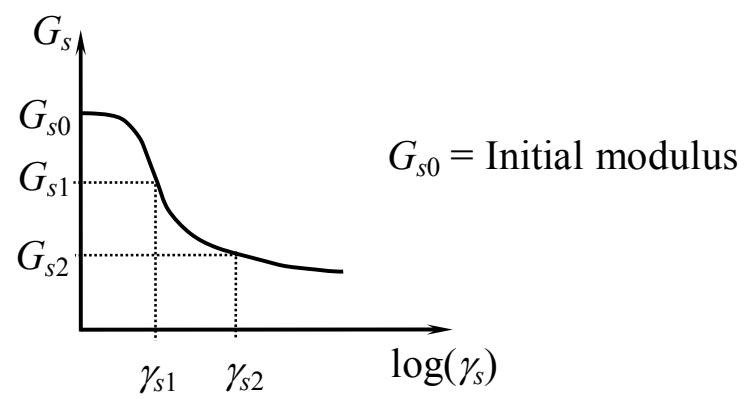

Figure 4-2 Typical Modulus Degradation Curve of Soil 
The relative fractions of elastic and plastic strains and the rate of modulus degradation with strain depend on the stress state and the soil state. By stress state we mean the vertical and lateral stresses acting on a soil element (the representative equivalent volume REV in mechanics terminology). Soil state, among other things, refers to the degree of packing of soil particles (i.e., whether the particles are densely or loosely packed). In order to describe the soil state, we define a few soil parameters, which are called the state parameters. Void ratio is one such state parameter which affects the soil stiffness. It describes how densely the soil particles are packed and is defined as the ratio of the volume of voids (pores) within a REV to the volume of solid soil particles within the same REV. The lower the void ratio, the denser the packing of soil particles is. Owing to denser soil packing, a soil mass with lower void ratio offers greater resistance to deformation and hence, the modulus is higher and the degradation with strain occurs at a slower rate. Dense sands and stiff clays have lower void ratio, and, in general, show higher stiffness and lower modulus degradation rate than loose sands and soft clays. However, the void ratio is not the only controlling factor, the stress state and the soil type also play a role in determining the stiffness and its change with strain. For the same soil with the same void ratio, a REV with a higher confining pressure (mean stress) has a higher stiffness and a faster degradation rate than a soil element with lower confining pressure. Again, elements of two different types of soil with the same void ratio and the same confining stress may have different stiffness and modulus degradation rates because the shape and size of the grains (these are called the intrinsic soil parameters) play some role in the deformation.

The highly nonlinear behavior of soil can be modeled by developing constitutive relationships using the concepts from classical, rate-independent plasticity (Lubliner 1990) if time effects on the stress-strain relationships can be neglected. In fact, such models exist and have been used in the analysis of foundations (Loukidis 2006), although such rigorous, plasticity-based analyses for laterally loaded piles are not known to have been performed. However, plasticity-based constitutive relationships, which explicitly account for soil yielding and strain/work hardening or softening, are strictly required when we are interested in estimating the collapse load more than in estimating the load-deflection response. For the calculation of pile deflection as a function of applied load, for the level of pile-head deflection (of the order of $25 \mathrm{~mm}$ ) we are interested in, a simpler approach of fitting a nonlinear stress-strain curve is sufficient. 
A nonlinear stress-strain curve can be incorporated in an elastic analysis by properly estimating the secant modulus (Figure 4-3) for a given level of strain (or stress). Many researchers in geotechnical engineering have used such an approach in the past; consequently, several nonlinear stress-strain relationships are available in the literature. A common way of representing the soil response is the hyperbolic model (Figure 4-3) (Kondner 1963, Fahey and Carter 1993):

$$
\frac{G_{s}}{G_{s 0}}=\frac{1}{1+\frac{\gamma_{s}}{\gamma_{s r}}}
$$

where $G_{s}$ is the shear modulus, $G_{s 0}$ is the initial (small-strain) shear modulus, $\gamma_{s}$ is the shear strain and $\gamma_{s r}$ is the reference shear strain defined as:

$$
\gamma_{s r}=\frac{\tau_{s \max }}{G_{s 0}}
$$

where $\tau_{\text {smax }}$ is the shear strength. Generally, the Mohr-Coulomb failure criterion (Lubliner 1990, Davis and Selvadurai 2002) is used to calculate soil shear strength, according to which:

$$
\tau_{s \max }=c+\sigma \tan \phi
$$

where $\tau_{s \max }$ is the shear strength on a plane (against slip) passing through a soil mass, $\sigma$ is the normal stress acting on the plane, $c$ is the cohesion (cementation, adhesion, bonding between soil particles) present in the soil and $\phi$ is the friction angle, which represents the frictional resistance of the soil (friction due to rolling, sliding and dislocation of soil particles). Note that the symbol $\phi$ used in the above equation is conventionally used to represent the shear resistance of soil due to friction, and has got no relationship with the function $\phi$ used in chapter 3. The friction angle $\phi$ to be used in equation (4-3) generally varies between $30^{\circ}$ and $45^{\circ}$ (values near the lower end of the range is applicable for clays while those near the upper end are applicable for sands). In soils, including clays, the cohesion $c$ is generally zero unless, in the rare case, there is cementation between particles (in which case, laboratory tests have to be performed to determine the cohesion). For clays, often a nonzero (fictitious) cohesion is assumed in which the $c$ and $\phi$ become curve-fitting parameters obtained from laboratory tests. However, if proper estimation of $\phi$ can be made (which is possible using laboratory or in situ tests), then no such estimation of fictitious cohesion is necessary. 


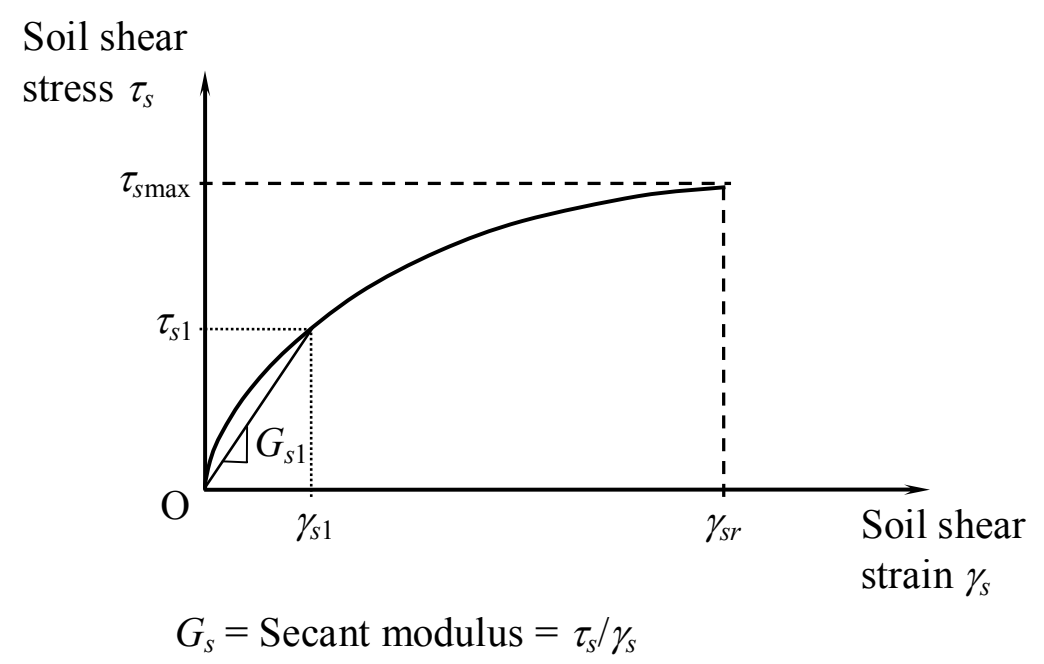

Figure 4-3 Hyperbolic Stress-Strain Plot of Soil

In equation (4-1a), $G_{s}$ represents the secant modulus. If at any instance of loading, $\tau_{s}$ is the shear stress acting on a plane that produces an engineering shear strain $\gamma_{s}$, then $G_{s}=\tau_{s} / \gamma_{s}$. Using equation (4-1a) and (4-2), $G_{s}$ in the hyperbolic model is expressed in terms of stress as (Fahey and Carter 1993):

$$
\frac{G_{s}}{G_{s 0}}=1-\frac{\tau}{\tau_{s \max }}
$$

The hyperbolic model, although simple, has been observed to not model the soil nonlinearity adequately. Consequently, Fahey and Carter (1993) modified the hyperbolic model as:

$$
\frac{G_{s}}{G_{s 0}}=1-f\left(\frac{\tau_{s}}{\tau_{s \max }}\right)^{g}
$$

where the fitting parameters $f$ and $g$ depend on the soil type. When expressed in terms of strain using equation (4-2), the $f-g$ model is written as:

$$
\frac{G_{s}}{G_{s 0}}=\frac{1}{1+f\left(\frac{G_{s}}{G_{s 0}}\right)^{g-1}\left(\frac{\gamma_{s}}{\gamma_{s r}}\right)^{g}}
$$

The parameters $f$ and $g$ have to be determined by fitting equation (4-4) to stress-strain plots obtained from laboratory or in situ tests. Based on a few experimental results, Fahey and Carter 
(1993) suggested values of $f$ ranging from 0.8 to 1.0 and of $g$ ranging from 0.25 to 1.0 . They further suggested that $f=0.98$ and $g=0.25$ give reasonably good results for Toyoura sand. Mayne (2000) suggested that $f=1$ and $g=0.3$ can be used for clays with reasonable accuracy.

Lee and Salgado (2000) extended the $f$ - $g$ model for three-dimensional stress state as:

$$
\frac{G_{s}}{G_{s 0}}=1-f\left(\frac{\sqrt{I I_{s}}-\sqrt{I I_{s 0}}}{\sqrt{I_{s \max }}-\sqrt{I I_{s 0}}}\right)^{g}
$$

where $I I_{S}$ is the second invariant of stress deviator tensor (it is a three-dimensional equivalent of shear stress $\tau$ ); $I I_{s 0}$ is the second invariant of the initial stress deviator tensor (it is a threedimensional equivalent of the initial shear stress present in the soil mass, even before the application of load, due to inherent stress anisotropy of the soil mass) and $I I_{s \max }$ is the maximum value of $I I_{s}$ and is related to the soil shear strength (it is a three-dimensional equivalent of the maximum shear stress $\tau_{s \max }$ ). If the effect of initial stresses can be neglected, then equation (45a) can be simplified to:

$$
\frac{G_{s}}{G_{s 0}}=1-f\left(\frac{\sqrt{I I_{s}}}{\sqrt{I I_{s \max }}}\right)^{g}
$$

$I I_{s \max }$ can be estimated by a suitable choice of a failure criterion. The Drucker-Prager failure criterion (Lubliner 1990) has been used for determining three-dimensional failure state; accordingly, $I I_{s \max }$ can be expressed as:

$$
\sqrt{I I_{s \max }}-\alpha I_{\sigma}-\kappa=0
$$

where $\alpha$ and $\kappa$ can be related to the Mohr-Coulomb $\phi$ and $c$ as:

$$
\begin{aligned}
& \alpha=\frac{2 \sin \phi}{\sqrt{3}(3-\sin \phi)} \\
& \kappa=\frac{6 c \cos \phi}{\sqrt{3}(3-\sin \phi)}
\end{aligned}
$$

Based on finite element analyses and experimental results, Lee and Salgado (2000) suggested that, for Ticino sand, $f$ ranges between 0.93 and 0.98 , and $g$ ranges between 0.15 and 0.32 .

In order to account for the dependence of shear modulus on confinement (mean stress), Lee and Salgado (2000) modified equation (4-5a) as: 


$$
\frac{G_{s}}{G_{s 0}}=\left[1-f\left(\frac{\sqrt{I I_{s}}-\sqrt{I I_{s 0}}}{\sqrt{I_{s \max }}-\sqrt{I I_{s 0}}}\right)^{g}\right]\left(\frac{I_{\sigma}}{I_{\sigma 0}}\right)^{n_{g}}
$$

where $I_{\sigma}$ is the first invariant of the stress tensor and is a measure of the confining stress; $I_{\sigma 0}$ is a measure of the initial confining stress (due to the presence of initial stresses), $n_{g}$ is a parameter that depends on the type of soil (intrinsic parameter).

The $f-g$ model and its three-dimensional modification discussed above are primarily stress dependent because the modulus can be determined directly (i.e., without iterations) by giving stress as input. Strain-dependent models are also available in the literature and, in fact, are more suitable for our analysis. One such widely used model was developed by Ishibashi and Zhang (1993), who proposed modulus degradation equations for sands based on a series of laboratory tests performed by various researchers:

$$
\frac{G_{s}}{G_{s 0}}=K(\gamma) \sigma_{m}^{q(\gamma)}
$$

where $\sigma_{m}$ is the mean stress $\left[=\left(\sigma_{11}+\sigma_{22}+\sigma_{33}\right) / 3\right]$ in $\mathrm{kPa}$, the parameter $K(\gamma)$ and the exponent $q(\gamma)$ depend on the engineering shear strain $\gamma$ as:

$$
\begin{aligned}
& K(\gamma)=0.5\left[1+\tanh \left\{\ln \left(\frac{0.000102}{\gamma}\right)^{0.492}\right\}\right] \\
& q(\gamma)=0.272\left[1-\tanh \left\{\ln \left(\frac{0.000556}{\gamma}\right)^{0.4}\right\}\right]
\end{aligned}
$$

Ishibashi and Zhang (1993) also worked on clays and proposed the following equations:

$$
\frac{G_{s}}{G_{s 0}}=K\left(\gamma, I_{p}\right) \sigma_{m}^{q(\gamma)}
$$

where the parameter $K\left(\gamma, I_{p}\right)$ and the exponent $q(\gamma)$ depend not only on the engineering shear strain $\gamma$ but also on the plasticity index $I_{p}$ (plasticity index is a measure of the water retention capacity of clay; it gives an approximate idea of how plastic or brittle a clay is):

$$
K\left(\gamma, I_{p}\right)=0.5\left[1+\tanh \left\{\ln \left(\frac{0.000102+f\left(I_{p}\right)}{\gamma}\right)^{0.492}\right\}\right]
$$




$$
q(\gamma)=0.272\left[1-\tanh \left\{\ln \left(\frac{0.000556}{\gamma}\right)^{0.4}\right\}\right] e^{-0.00145 I_{p}^{1.3}}
$$

The parameter $f\left(I_{p}\right)=0$ is given by:

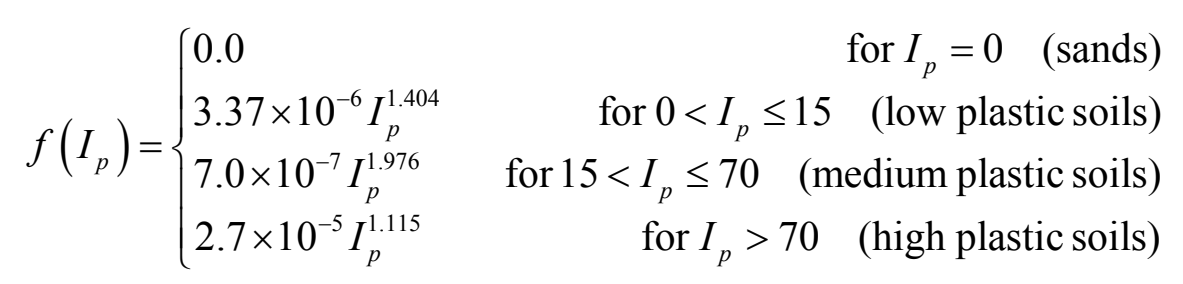

For three-dimensional problems, $\gamma$ should be the engineering octahedral shear strain (i.e., twice the octahedral shear strain).

Isotropic elastic materials are defined by two constants (e.g., $\lambda_{s}$ and $G_{s}$; or $K_{s}$ and $G_{s}$; or $E_{s}$ and $\left.v_{s}\right)$. Both constants change with the application of load if a nonlinear elastic process is considered. The variation of $G_{s}$ with strain or stress is well documented in the literature (as shown above), but the variation of the other constant is not well studied. Fahey and Carter (1993) suggested that the ratio of $K_{s}$ and $G_{s}$ can be assumed to remain constant during the loading process (i.e., $K_{s}$ and $G_{s}$ both degrade maintaining $K_{s} / G_{s}$ a constant). Naylor et al. (1981) proposed a linear relationship between the bulk modulus $K_{s}$ and the mean stress $\sigma_{m}$. Lee and Salgado (2000) suggested a nonlinear dependence of $K_{s}$ on the mean stress, according to which:

$$
\frac{K_{s}}{P_{a}}=D_{s}\left(\frac{\sigma_{m}}{P_{a}}\right)^{n_{k}}
$$

where $P_{a}$ is a reference stress $(100 \mathrm{kPa}), \sigma_{m}$ is the mean stress, $D_{s}$ is a material constant which can be determined from the initial bulk modulus $K_{s 0}$ and the initial mean stress $\sigma_{m 0}$ :

$$
D_{s}=K_{s 0} \sigma_{m 0}^{-n_{k}} P_{a}^{\left(n_{k}-1\right)}
$$

in which the initial bulk modulus $K_{s 0}$ can be obtained from the initial shear modulus $G_{s 0}$ and initial Poisson's ratio $v_{s 0}\left(K_{s}=E_{s} /\left\{3\left(1-2 v_{s}\right)\right\}=\lambda_{s}+(2 / 3) G_{s}=2 G_{s}\left(1+v_{s}\right) /\left\{3\left(1-2 v_{s}\right)\right\}\right)$. Lee and Salgado (2000) suggested a value of 0.5 for $n_{k}$. The Poisson's ratio varies within a small range and a fair estimate of the Poisson's ratio, for different soils, can be made from the available literature (Selvadurai 1979, Salgado 2008). Salgado (2008) suggested that $v_{s 0}$ varies between 0.1 and 0.2 for sands. For clays, a value between 0.3 and 0.5 is a reasonable estimate of $v_{s 0}$ depending on the degree of saturation of clay. The greater the degree of saturation, the more 
incompressible the clay initially is, the greater the value of $v_{s 0}$ is. For the case of drained loading, $v_{s 0}$ would be in the 0.2 to 0.3 range.

The initial shear modulus $G_{s 0}$ has been found to be related, among other factors, to the (initial) void ratio $e_{0}$ and to the (initial) mean stress $\sigma_{m 0}$ (Hardin and Black 1966, 1968, Hardin and Drnevich 1972, Hardin 1978). For sands, $G_{s 0}$ can be estimated from (Salgado 2008):

$$
\frac{G_{s 0}}{P_{a}}=C_{g} \frac{\left(e_{g}-e_{0}\right)^{2}}{1+e_{0}}\left(\frac{\sigma_{m 0}}{P_{a}}\right)^{n_{g}}
$$

where $C_{g}, e_{g}$ and $n_{g}$ depend on the soil type (intrinsic soil properties). $C_{g}=650, e_{g}=2.17$ and $n_{g}$ $=0.45$ can be used for sands if no specific data are available. For clays, the following equation can be used (Salgado 2008):

$$
\frac{G_{s 0}}{P_{a}}=C_{g} \frac{\left(e_{g}-e_{0}\right)^{2}}{1+e_{0}}\left(\frac{\sigma_{m 0}}{P_{a}}\right)^{n_{g}} \mathrm{OCR}^{m_{g}}
$$

where OCR is the overconsolidation ratio (it is defined as the ratio of the maximum stress experienced by a soil deposit to the present existing stress). OCR plays an important role in deciding the stiffness in clayey soils in addition to the void ratio and the mean stress. The exponent $m_{g}$ can be assumed to be equal to 0.5 . The exponent $n_{g}$ of equation (4-20) is the same exponent used in equation (4-10).

Viggiani and Atkinson (1995) suggested a similar equation for clays:

$$
\frac{G_{s 0}}{p_{a}}=A\left(\frac{\sigma_{m 0}}{p_{a}}\right)^{n_{g}} \mathrm{OCR}^{m_{g}}
$$

where the reference stress $p_{a}$ is equal to $1 \mathrm{kPa}$, and the parameters $A, n_{g}$, and $m_{g}$ can be estimated from the plasticity index $I_{p}$ by using the following equations (Foye, Basu and Prezzi, personal communication):

$$
\begin{aligned}
& A=3790 e^{-0.045 I_{p}} \\
& n_{g}=0.109 \ln I_{p}+0.4374 \text { for } I_{p}>5 \\
& m_{g}=0.0015 I_{p}+0.1863 \text { for } I_{p}>5
\end{aligned}
$$

The stresses to be used in all the above equations should be effective stresses. 


\subsection{Nonlinear Pile Analysis}

\subsubsection{Problem Description}

We consider the same multi-layered problem described in chapter 3 with the added condition that the soil within each layer is not linear elastic but nonlinear elastic (i.e., the constants $\lambda_{s}$ and $G_{s}$ within each layer are not constants but is a function of soil strain or stress). When a pile is loaded laterally, the displacements and strains at the pile-soil interface are the same for both the pile and the soil if perfect soil-pile contact is assumed. However, at any radial distance from the pile, the soil displacements and strains are less than the corresponding values at the pile-soil interface; they in fact decrease gradually with increasing radial distance from the pile (Figure 4-4). For any constitutive relationship, the modulus degradation depends on the level of strain (i.e., the higher the strain, the greater the degradation is) (Figure 4-2); therefore, at any particular instance of the loading process, the soil modulus varies in the radial direction (Figure 4-4). Assuming the soil deposit to be homogeneous in its initial state, the modulus has a minimum value at the pile-soil interface (where the strain is at a maximum) and increases with increasing $r$. The modulus varies in the tangential direction as well, because the applied force $F_{a}$ and moment $M_{a}$ create displacement and strain fields that are not axisymmetric (Figure 4-5). Consequently, the elastic constants (e.g., the Lame's constants) within each layer, at any instance

of loading, are functions of both $r$ and $\theta$. The elastic constants are functions of $z$ as well, but its variation along $z$ is taken into account by considering the soil layering. 


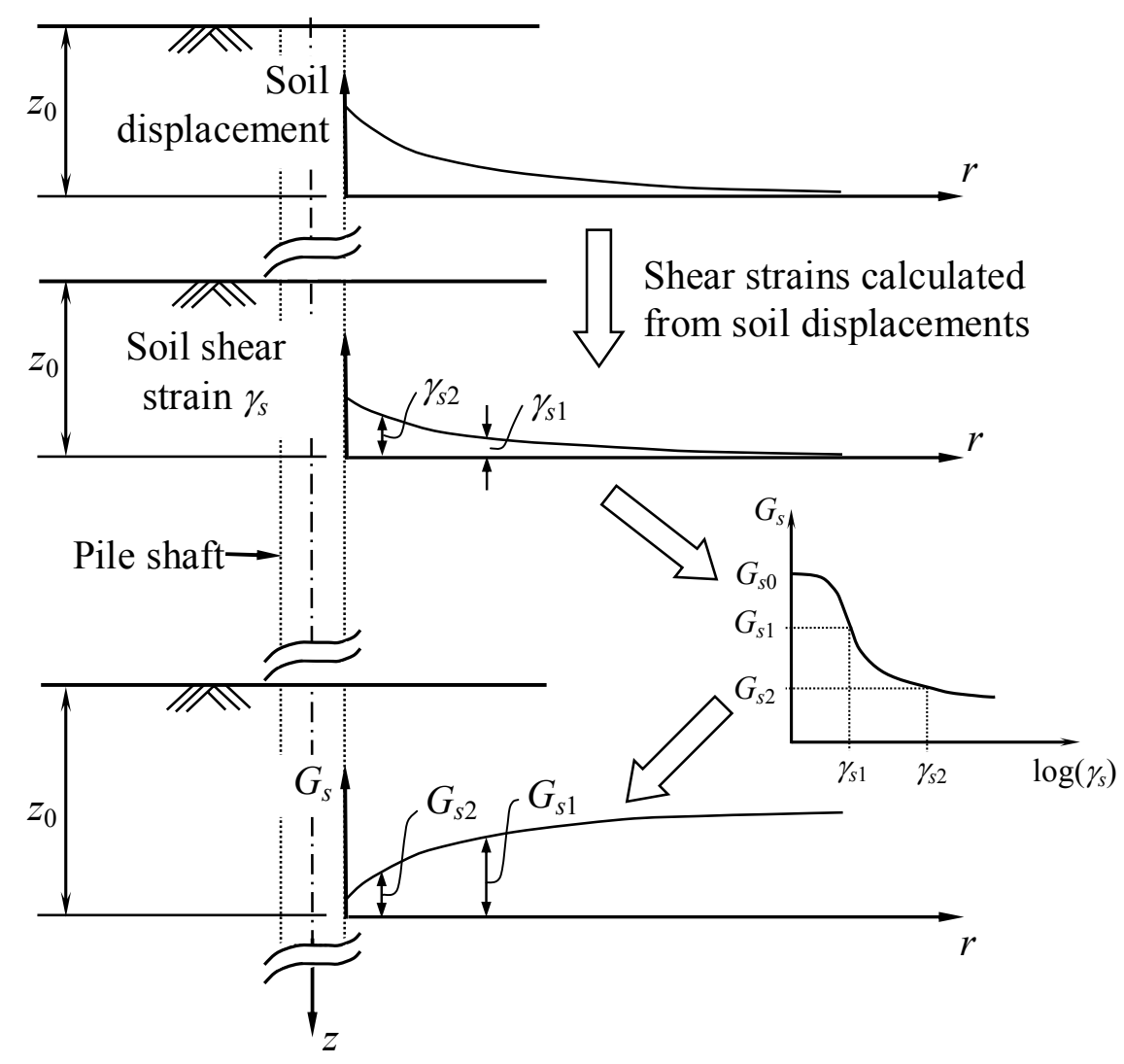

Figure 4-4 Variations of Soil Displacement, Strain and Modulus, at a given Depth $z_{0}$, with Radial Distance $r$ from the Pile

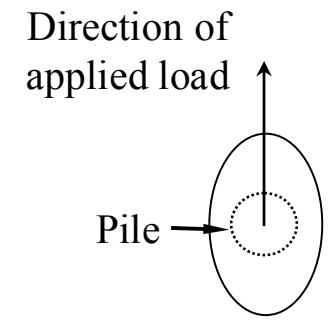

Variation of soil displacement surrounding the pile

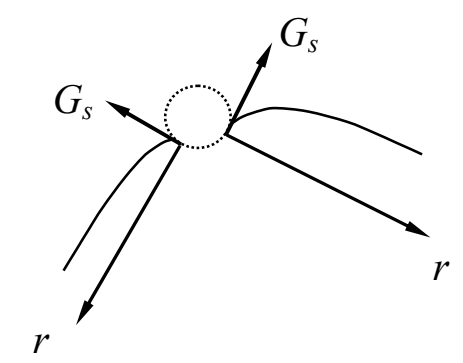

Different variations of soil modulus surrounding the pile

Figure 4-5 Tangential Variation of Soil Displacement and Modulus Surrounding a Pile 
In the derivation of the governing differential equations that can capture the nonlinear soil response, the soil within each layer is assumed to be elastic and isotropic, but heterogeneous (with respect to $r$ and $\theta$ but not with respect to $z$ ) with no sliding or separation between the soil layers or between the pile and the soil. By solving the equations (valid for elastic, heterogeneous soil) recurrently for different magnitudes of load (with appropriate values and variations of soil modulus), the analysis is capable of tracing the nonlinear progression of pile deflection (due to soil nonlinearity) with increasing applied load.

\subsubsection{Principle of Virtual Work}

Applying the principle of virtual work to the pile-soil system, we get:

$$
\begin{gathered}
E_{p} I_{p} \int_{0}^{L_{p}}\left(\frac{d^{2} w}{d z^{2}}\right) \delta\left(\frac{d^{2} w}{d z^{2}}\right) d z+\int_{0}^{\infty} \int_{0}^{2 \pi} \int_{r_{p}}^{\infty} \sigma_{i j} \delta \varepsilon_{i j} r d r d \theta d z+\int_{L_{p}}^{\infty} \int_{0}^{2 \pi} \int_{0}^{r_{p}} \sigma_{i j} \delta \varepsilon_{i j} r d r d \theta d z \\
-\left.F_{a} \delta w\right|_{z=0}+\left.M_{a} \delta\left(\frac{d w}{d z}\right)\right|_{z=0}=0
\end{gathered}
$$

In the above equation, the first integral represents the internal virtual work of the pile. The second and the third integrals represent the internal virtual work of the soil continuum (summation is implied by index repetition); in particular, the third integral represents the soil column of radius $r_{p}$ extending from below the pile to infinity downward while the second integral represents the rest of the soil mass. The remaining two terms represent the external virtual work (due to the presence of the applied force and moment).

We assume the same displacement field as given in equation (3-2); therefore the strains are related to the displacements through equation (3-3). We further make the assumption that at any instance of loading, the total accumulated strain at any point within the soil mass is related linearly to the total accumulated stress at that point, and that the linear relationship between the different components of accumulated strain and stress follow elasticity (equation (3-4)). Relating the stresses to strains using secant modulus, and relating strains to displacements (following a similar procedure as that of chapter 3), we get: 


$$
\begin{aligned}
\int_{0}^{L_{p}} E_{p} I_{p} \frac{d^{2} w}{d z^{2}} \delta\left(\frac{d^{2} w}{d z^{2}}\right) d z+\int_{0}^{\infty} \int_{r_{p}}^{\infty} \int_{0}^{2 \pi}\left[\left(\lambda_{s}+2 G_{s}\right) w \delta w\left(\frac{d \phi_{r}}{d r}\right)^{2} \cos ^{2} \theta\right. \\
+\left(\lambda_{s}+2 G_{s}\right) w^{2} \cos ^{2} \theta\left(\frac{d \phi_{r}}{d r}\right) \delta\left(\frac{d \phi_{r}}{d r}\right)+2 \lambda_{s} w \delta w \cos ^{2} \theta \frac{1}{r} \frac{d \phi_{r}}{d r}\left(\phi_{r}-\phi_{\theta}\right) \\
+\lambda_{s} w^{2} \cos ^{2} \theta \frac{1}{r}\left(\phi_{r}-\phi_{\theta}\right) \delta\left(\frac{d \phi_{r}}{d r}\right)+\lambda_{s} w^{2} \cos ^{2} \theta \frac{1}{r} \frac{d \phi_{r}}{d r} \delta \phi_{r} \\
+\lambda_{s} w^{2} \cos ^{2} \theta \frac{1}{r} \frac{d \phi_{r}}{d r} \delta \phi_{\theta}+\left(\lambda_{s}+2 G_{s}\right) w \delta w \cos ^{2} \theta \frac{1}{r^{2}}\left(\phi_{r}-\phi_{\theta}\right)^{2} \\
+\left(\lambda_{s}+2 G_{s}\right) w^{2} \cos ^{2} \theta \frac{1}{r^{2}}\left(\phi_{r}-\phi_{\theta}\right) \delta \phi_{r}-\left(\lambda_{s}+2 G_{s}\right) w^{2} \cos ^{2} \theta \frac{1}{r^{2}}\left(\phi_{r}-\phi_{\theta}\right) \delta \phi_{\theta} \\
+G_{s} w \delta w \sin ^{2} \theta \frac{1}{r^{2}}\left(\phi_{r}-\phi_{\theta}\right)^{2}+2 G_{s} w \delta w \sin ^{2} \theta \frac{1}{r} \frac{d \phi_{\theta}}{d r}\left(\phi_{r}-\phi_{\theta}\right) \\
+G_{s} w^{2} \sin ^{2} \theta \frac{1}{r^{2}}\left(\phi_{r}-\phi_{\theta}\right) \delta \phi_{r} \\
+G_{s}\left(\frac{d w}{d z}\right)^{2} \sin ^{2} \theta \phi_{\theta} \delta \phi_{\theta} w^{2} \sin ^{2} \theta \frac{1}{r^{2}}\left(\phi_{r}-\phi_{\theta}\right) \delta \phi_{\theta}+G_{s} w^{2} \sin ^{2} \theta \frac{1}{r} \frac{d \phi_{\theta}}{d r} \delta \phi_{r}-G_{s} w^{2} \sin ^{2} \theta \frac{1}{r} \frac{d \phi_{\theta}}{d r} \delta \phi_{\theta} \\
+G_{s}\left(\frac{d w}{d z}\right)^{2} \cos ^{2} \theta \phi_{r} \delta \phi_{r}+G_{s} \frac{d w}{d z} \delta\left(\frac{d w}{d z}\right) \phi_{\theta}^{2} \sin ^{2} \theta \\
+G_{s} w \delta w \sin ^{2} \theta\left(\frac{d \phi_{\theta}}{d r}\right)^{2}+G_{s} w^{2} \sin ^{2} \theta \frac{d \phi_{\theta}}{d r} \delta\left(\frac{d \phi_{\theta}}{d r}\right) \\
+G_{s} w^{2} \sin ^{2} \theta \frac{1}{r}\left(\phi_{r}-\phi_{\theta}\right) \delta\left(\frac{d \phi_{\theta}}{d r}\right)+G_{s} \frac{d w}{d z} \delta\left(\frac{d w}{d z}\right) \phi_{r}^{2} \cos ^{2} \theta \\
+
\end{aligned}
$$

where $\lambda_{s}=\lambda_{s}(r, \theta)$ and $G_{s}=G_{s}(r, \theta)$ represent the secant modulus. For the infinitely long column of soil (having the same radius as that of the pile) beneath the pile, $\lambda_{s}$ and $G_{s}$ are assumed to be constant.

We consider separately the variations $\delta w, \delta \phi_{r}$ and $\delta \phi_{\theta}$ of the functions $w(z), \phi_{r}(r)$ and $\phi_{\theta}(r)$ (since the variations are linearly independent). We collect the terms associated with $\delta w, \delta \phi_{r}$ and $\delta \phi_{\theta}$ and equate each to zero so that equation (4-24b) is satisfied. As in chapter 3, we 
consider the variation of $w$ over two separate sub-domains: $0 \leq z \leq L_{p}$ and $L_{p} \leq z<\infty$. For the functions $\phi_{r}$ and $\phi_{\theta}$, we consider the variations over the domain $r_{p} \leq r<\infty$.

\subsubsection{Soil Displacement}

We first consider the variation on $\phi_{r}(r)$. From equation (4-24b), we collect all the terms associated with $\delta \phi_{r}$ and equate their summation to zero. By doing so, we ensure that the terms collectively satisfy the principle of virtual work. The expression containing the pertinent $\phi_{r^{-}}$ related terms is written as:

$$
\begin{aligned}
& \int_{r_{p}}^{\infty}\left[m_{s 1} \frac{d \phi_{r}}{d r} \delta\left(\frac{d \phi_{r}}{d r}\right)+m_{s 3} \frac{1}{r}\left(\phi_{r}-\phi_{\theta}\right) \delta\left(\frac{d \phi_{r}}{d r}\right)+m_{s 3} \frac{1}{r} \frac{d \phi_{r}}{d r} \delta \phi_{r}+m_{s 1} \frac{1}{r^{2}}\left(\phi_{r}-\phi_{\theta}\right) \delta \phi_{r}\right. \\
& \left.+m_{s 2} \frac{1}{r^{2}}\left(\phi_{r}-\phi_{\theta}\right) \delta \phi_{r}+m_{s 2} \frac{1}{r} \frac{d \phi_{\theta}}{d r} \delta \phi_{r}+n_{s 1} \phi_{r} \delta \phi_{r}\right] d r=0
\end{aligned}
$$

where

$$
\begin{aligned}
& m_{s 1}(r)=\int_{0}^{\infty} \int_{0}^{2 \pi}\left(\lambda_{s}+2 G_{s}\right) w^{2} \cos ^{2} \theta r d \theta d z=\sum_{i=1}^{n} \int_{H_{i-1}}^{H_{i}} \int_{0}^{2 \pi}\left(\lambda_{s i}+2 G_{s i}\right) w_{i}^{2} \cos ^{2} \theta r d \theta d z \\
& m_{s 2}(r)=\int_{0}^{\infty} \int_{0}^{2 \pi} G_{s} w^{2} \sin ^{2} \theta r d \theta d z=\sum_{i=1}^{n} \int_{H_{i-1}}^{H_{i}} \int_{0}^{2 \pi} G_{s i} w_{i}^{2} \sin ^{2} \theta r d \theta d z \\
& m_{s 3}(r)=\int_{0}^{\infty} \int_{0}^{2 \pi} \lambda_{s} w^{2} \cos ^{2} \theta r d \theta d z=\sum_{i=1}^{n} \int_{H_{i-1}}^{H_{i}} \int_{0}^{2 \pi} \lambda_{s i} w_{i}^{2} \cos ^{2} \theta r d \theta d z \\
& n_{s 1}(r)=\int_{0}^{\infty} \int_{0}^{2 \pi} G_{s}\left(\frac{d w}{d z}\right)^{2} \cos ^{2} \theta r d \theta d z=\sum_{i=1}^{n} \int_{H_{i-1}}^{H_{i}} \int_{0}^{2 \pi} G_{s i}\left(\frac{d w_{i}}{d z}\right)^{2} \cos ^{2} \theta r d \theta d z
\end{aligned}
$$

In equations (4-26) through (4-29), the subscript $i$ represents the $i^{\text {th }}$ layer of the multi-layered continuum; $w_{i}$ represents the function $w(z)$ in the $i^{\text {th }}$ layer with $\left.w_{i}\right|_{z=H_{i}}=\left.w_{i+1}\right|_{z=H_{i}}$; and $H_{n}=\infty$. Further simplification of equation (4-25a) by performing integration by parts of the terms containing $\delta\left(\frac{d \phi_{r}}{d r}\right)$ produces: 


$$
\begin{aligned}
\int_{r_{p}}^{\infty}\left[-m_{s 1} \frac{d^{2} \phi_{r}}{d r^{2}}-\frac{d m_{s 1}}{d r} \frac{d \phi_{r}}{d r}-\frac{1}{r} \frac{d m_{s 3}}{d r} \phi_{r}+\left(m_{s 1}+m_{s 2}+m_{s 3}\right) \frac{\phi_{r}}{r^{2}}+n_{s 1} \phi_{r}+\frac{1}{r} \frac{d m_{s 3}}{d r} \phi_{\theta}\right. \\
\left.+\frac{m_{s 2}+m_{s 3}}{r} \frac{d \phi_{\theta}}{d r}-\left(m_{s 1}+m_{s 2}+m_{s 3}\right) \frac{\phi_{\theta}}{r^{2}}\right] \delta \phi_{r} d r+\left.m_{s 1} \frac{d \phi_{r}}{d r} \delta \phi_{r}\right|_{r_{p}} ^{\infty} \\
+\left.m_{s 3} \frac{1}{r}\left(\phi_{r}-\phi_{\theta}\right) \delta \phi_{r}\right|_{r_{p}} ^{\infty}=0
\end{aligned}
$$

Since the function $\phi_{r}$ is not known a priori within the domain $r_{p} \leq r<\infty, \phi_{r}$ has a non-zero variation (i.e., $\delta \phi_{r} \neq 0$ ). Therefore, equation (4-25b) is satisfied if and only if the terms within the integral in the left-hand-side are collectively equal to zero (because $\delta \phi_{r} \neq 0$ ); this gives the differential equation of $\phi_{r}$ :

$$
\begin{gathered}
-m_{s 1} \frac{d^{2} \phi_{r}}{d r^{2}}-\frac{d m_{s 1}}{d r} \frac{d \phi_{r}}{d r}-\frac{1}{r} \frac{d m_{s 3}}{d r} \phi_{r}+\left(m_{s 1}+m_{s 2}+m_{s 3}\right) \frac{\phi_{r}}{r^{2}}+n_{s 1} \phi_{r}+\frac{1}{r} \frac{d m_{s 3}}{d r} \phi_{\theta} \\
+\frac{m_{s 2}+m_{s 3}}{r} \frac{d \phi_{\theta}}{d r}-\left(m_{s 1}+m_{s 2}+m_{s 3}\right) \frac{\phi_{\theta}}{r^{2}}=0
\end{gathered}
$$

which, when rearranged, produces:

$$
\begin{aligned}
\frac{d^{2} \phi_{r}}{d r^{2}}+\frac{1}{m_{s 1}} \frac{d m_{s 1}}{d r} & \frac{d \phi_{r}}{d r}-\left\{\frac{1}{r^{2}} \frac{m_{s 1}+m_{s 2}+m_{s 3}}{m_{s 1}}-\frac{1}{r} \frac{1}{m_{s 1}} \frac{d m_{s 3}}{d r}+\frac{n_{s 1}}{m_{s 1}}\right\} \phi_{r} \\
& =\frac{m_{s 2}+m_{s 3}}{m_{s 1}} \frac{1}{r} \frac{d \phi_{\theta}}{d r}-\left\{\frac{1}{r^{2}} \frac{m_{s 1}+m_{s 2}+m_{s 3}}{m_{s 1}}-\frac{1}{r} \frac{1}{m_{s 1}} \frac{d m_{s 3}}{d r}\right\} \phi_{\theta}
\end{aligned}
$$

At $r=\infty, \phi_{r}=0$ because the displacements within the soil due to the pile deflection must be negligible at sufficiently large distances from the pile. Hence, $\phi_{r}$ is known at $r=\infty$ and the first variation of $\phi_{r}$ at that point is equal to zero (i.e., $\delta \phi_{r}=0$ at $r=\infty$ ). This satisfies equation (4$25 \mathrm{~b})$ and produces the boundary conditions for equation (4-30). The other boundary condition imposed is $\phi_{r}=1$ at $r=r_{p}$ (which makes $\delta \phi_{r}=0$ at $r=r_{p}$ and satisfies equation (4-25b) as well).

We now consider the variation on $\phi_{\theta}(r)$. From equation (4-24b) we collect the terms containing $\delta \phi_{\theta}$ and $\delta\left(\frac{d \phi_{\theta}}{d r}\right)$ and, following a similar procedure as for $\phi_{r}$, we get the following governing differential equation for $\phi_{\theta}$ : 


$$
\begin{aligned}
\frac{d^{2} \phi_{\theta}}{d r^{2}}+\frac{1}{m_{s 2}} \frac{d m_{s 2}}{d r} \frac{d \phi_{\theta}}{d r} & -\left\{\frac{1}{r^{2}} \frac{m_{s 1}}{m_{s 2}}+\frac{1}{r} \frac{1}{m_{s 2}} \frac{d m_{s 2}}{d r}+\frac{n_{s 2}}{m_{s 2}}\right\} \phi_{\theta} \\
& =-\frac{m_{s 2}+m_{s 3}}{m_{s 2}} \frac{1}{r} \frac{d \phi_{r}}{d r}-\left\{\frac{1}{r^{2}} \frac{m_{s 1}}{m_{s 2}}+\frac{1}{r} \frac{1}{m_{s 2}} \frac{d m_{s 2}}{d r}\right\} \phi_{r}
\end{aligned}
$$

with the boundary conditions that $\phi_{\theta}=0$ at $r=\infty$ and $\phi_{\theta}=1$ at $r=r_{p}$, where

$$
n_{s 2}(r)=\int_{0}^{\infty} \int_{0}^{2 \pi} G_{s}\left(\frac{d w}{d z}\right)^{2} \sin ^{2} \theta r d \theta d z=\sum_{i=1}^{n} \int_{H_{i-1}}^{H_{i}} \int_{0}^{2 \pi} G_{s i}\left(\frac{d w_{i}}{d z}\right)^{2} \sin ^{2} \theta r d \theta d z
$$

\subsubsection{Finite Difference Solution for Soil Displacements}

The differential equations (4-30b) and (4-31) for $\phi_{r}$ and $\phi_{\theta}$ are interdependent, and following similar steps as done in chapter 3, can be solved by the finite difference method. Using the central-difference scheme, equations (4-30b) and (4-31) can be respectively written as:

$$
\begin{aligned}
& \frac{\phi_{r}^{j+1}-2 \phi_{r}^{j}+\phi_{r}^{j-1}}{\Delta r^{2}}+\frac{1}{m_{s 1}^{j}} \frac{m_{s 1}^{j+1}-m_{s 1}^{j-1}}{2 \Delta r} \frac{\phi_{r}^{j+1}-\phi_{r}^{j-1}}{2 \Delta r}-\left[\frac{1}{r_{j}^{2}} \frac{m_{s 1}^{j}+m_{s 2}^{j}+m_{s 3}^{j}}{m_{s 1}^{j}}\right. \\
& \left.-\frac{1}{r_{j}} \frac{1}{m_{s 1}^{j}} \frac{m_{s 3}^{j+1}-m_{s 3}^{j-1}}{2 \Delta r}+\frac{n_{s 1}^{j}}{m_{s 1}^{j}}\right] \phi_{r}^{j}=\frac{m_{s 2}^{j}+m_{s 3}^{j}}{m_{s 1}^{j}} \frac{1}{r_{j}} \frac{\phi_{\theta}^{j+1}-\phi_{\theta}^{j-1}}{2 \Delta r} \\
& -\left[\frac{1}{r_{j}^{2}} \frac{m_{s 1}^{j}+m_{s 2}^{j}+m_{s 3}^{j}}{m_{s 1}^{j}}-\frac{1}{r_{j}} \frac{1}{m_{s 1}^{j}} \frac{m_{s 3}^{j+1}-m_{s 3}^{j-1}}{2 \Delta r}\right] \phi_{\theta}^{j} \\
& \frac{\phi_{\theta}^{j+1}-2 \phi_{\theta}^{j}+\phi_{\theta}^{j-1}}{\Delta r^{2}}+\frac{1}{m_{s 2}^{j}} \frac{m_{s 2}^{j+1}-m_{s 2}^{j-1}}{2 \Delta r} \frac{\phi_{\theta}^{j+1}-\phi_{\theta}^{j-1}}{2 \Delta r}-\left[\frac{1}{r_{j}^{2}} \frac{m_{s 1}^{j}}{m_{s 2}^{j}}+\frac{1}{r_{j}} \frac{1}{m_{s 2}^{j}} \frac{m_{s 2}^{j+1}-m_{s 2}^{j-1}}{2 \Delta r}\right. \\
& \left.+\frac{n_{s 2}^{j}}{m_{s 2}^{j}}\right] \phi_{\theta}^{j}=-\frac{m_{s 2}^{j}+m_{s 3}^{j}}{m_{s 2}^{j}} \frac{1}{r_{j}} \frac{\phi_{r}^{j+1}-\phi_{r}^{j-1}}{2 \Delta r}-\left[\frac{1}{r_{j}^{2}} \frac{m_{s 1}^{j}}{m_{s 2}^{j}}+\frac{1}{r_{j}} \frac{1}{m_{s 2}^{j}} \frac{m_{s 2}^{j+1}-m_{s 2}^{j-1}}{2 \Delta r}\right] \phi_{r}^{j}
\end{aligned}
$$

where $j$ represents the $j^{\text {th }}$ node and $\Delta r$ is the step length of the finite difference discretization described in chapter 6 for obtaining $\phi_{r}$ and $\phi_{\theta}$ (Figure 3-4).

The finite difference solution procedure is similar to that described in chapter 3 . Equations (4-33) and (4-34), when applied to the discretized nodes (except the $1^{\text {st }}$ and the last $\left(m^{\text {th }}\right)$ node, at which the values of $\phi_{r}$ and $\phi_{\theta}$ are known from the boundary conditions) produce two sets of simultaneous equations. Each equation set contains $m-2$ equations (there are 
altogether $m$ nodes, but we need to determine $\phi_{r}$ and $\phi_{\theta}$ for the second through the $(m-1)^{\text {th }}$ nodes), represented by the following matrix equation:

$$
[K]\{\delta\}=\{F\}
$$

where $[K]_{(m-2) \times(m-2)}$ represents the left-hand-side tridiagonal matrix containing the coefficients of $\phi_{r}{ }^{j}$ and $\phi_{\theta}{ }^{j}$ (see below); $\{\delta\}_{(m-2) \times 1}$ represents the unknown vector of nodal $\phi_{r}$ or $\phi_{\theta}$ values; and $\{F\}_{(m-2) \times 1}$ represents the right-hand side vector.

When $[K]$ is formed from equation (4-33) (i.e., when $\phi_{r}$ is the unknown), the non-zero elements of the $l^{\text {th }}$ row of $[K]$ are given by:

$$
\begin{aligned}
& {[K]_{l, l-1}=\frac{1}{\Delta r^{2}}-\frac{1}{m_{s 1}^{j}} \frac{m_{s 1}^{j+1}-m_{s 1}^{j-1}}{2 \Delta r} \frac{1}{2 \Delta r}} \\
& {[K]_{l, l}=-\frac{2}{\Delta r^{2}}-\frac{1}{r_{j}^{2}} \frac{m_{s 1}^{j}+m_{s 2}^{j}+m_{s 3}^{j}}{m_{s 1}^{j}}+\frac{1}{r_{j}} \frac{1}{m_{s 1}^{j}} \frac{m_{s 3}^{j+1}-m_{s 3}^{j-1}}{2 \Delta r}-\frac{n_{s 1}^{j}}{m_{s 1}^{j}}} \\
& {[K]_{l, l+1}=\frac{1}{\Delta r^{2}}+\frac{1}{m_{s 1}^{j}} \frac{m_{s 1}^{j+1}-m_{s 1}^{j-1}}{2 \Delta r} \frac{1}{2 \Delta r}}
\end{aligned}
$$

where $l=j-1$. The $l^{\text {th }}$ row of the corresponding $\{F\}$ vector is given by:

$$
\{F\}_{l}=\frac{m_{s 2}^{j}+m_{s 3}^{j}}{m_{s 1}^{j}} \frac{1}{r_{j}} \frac{\phi_{\theta}^{j+1}-\phi_{\theta}^{j-1}}{2 \Delta r}-\left[\frac{1}{r_{j}^{2}} \frac{m_{s 1}^{j}+m_{s 2}^{j}+m_{s 3}^{j}}{m_{s 1}^{j}}-\frac{1}{r_{j}} \frac{1}{m_{s 1}^{j}} \frac{m_{s 3}^{j+1}-m_{s 3}^{j-1}}{2 \Delta r}\right] \phi_{\theta}^{j}
$$

The boundary conditions $\left(\phi_{r}=1\right.$ at node 1 and $\phi_{r}=0$ at node $m$ ) modify the first and the $(m-2)^{\text {th }}$ rows of $\{F\}$, because $\phi_{r}^{j-1}=1$ for node 2 (which corresponds to the first row of $\{F\}$ ) and $\phi_{r}^{j+1}=$ 0 for node $m-1$ (which corresponds to the last row of $\{F\}$ ). These modified equations for nodes 2 and $m-1$ are given by:

$$
\begin{gathered}
\{F\}_{1}=-\frac{1}{\Delta r^{2}}+\frac{1}{m_{s 1}^{(2)}} \frac{m_{s 1}^{(3)}-m_{s 1}^{(1)}}{2 \Delta r} \frac{1}{2 \Delta r}+\frac{m_{s 2}^{(2)}+m_{s 3}^{(2)}}{m_{s 1}^{(2)}} \frac{1}{r_{2}} \frac{\phi_{\theta}^{(3)}-1}{2 \Delta r} \\
-\left[\frac{1}{r_{2}^{2}} \frac{m_{s 1}^{(2)}+m_{s 2}^{(2)}+m_{s 3}^{(2)}}{m_{s 1}^{(2)}}-\frac{1}{r_{2}} \frac{1}{m_{s 1}^{(2)}} \frac{m_{s 3}^{(3)}-m_{s 3}^{(3)}}{2 \Delta r}\right] \phi_{\theta}^{(2)} \\
\{F\}_{m-2}=\frac{m_{s 2}^{m-1}+m_{s 3}^{m-1}}{m_{s 1}^{m-1}} \frac{1}{r_{m-1}} \frac{-\phi_{\theta}^{m-2}}{2 \Delta r}-\left[\frac{1}{r_{m-1}^{2}} \frac{m_{s 1}^{m-1}+m_{s 2}^{m-1}+m_{s 3}^{m-1}}{m_{s 1}^{m-1}}\right. \\
\left.-\frac{1}{r_{m-1}} \frac{1}{m_{s 1}^{m-1}} \frac{m_{s 3}^{m}-m_{s 3}^{m-2}}{2 \Delta r}\right] \phi_{\theta}^{m-1}
\end{gathered}
$$


The non-zero elements of $[K]$, when formed from equation (4-34) (i.e., when $\phi_{\theta}$ is the unknown), are given by:

$$
\begin{aligned}
& {[K]_{l, l-1}=\frac{1}{\Delta r^{2}}-\frac{1}{m_{s 2}^{j}} \frac{m_{s 2}^{j+1}-m_{s 2}^{j-1}}{2 \Delta r} \frac{1}{2 \Delta r}} \\
& {[K]_{l, l}=-\frac{2}{\Delta r^{2}}-\frac{1}{r_{j}^{2}} \frac{m_{s 1}^{j}}{m_{s 2}^{j}}-\frac{1}{r_{j}} \frac{1}{m_{s 2}^{j}} \frac{m_{s 2}^{j+1}-m_{s 2}^{j-1}}{2 \Delta r}-\frac{n_{s 2}^{j}}{m_{s 2}^{j}}} \\
& {[K]_{l, l+1}=\frac{1}{\Delta r^{2}}+\frac{1}{m_{s 2}^{j}} \frac{m_{s 2}^{j+1}-m_{s 2}^{j-1}}{2 \Delta r} \frac{1}{2 \Delta r}}
\end{aligned}
$$

The $l^{\text {th }}$, first and $(m-2)^{\text {th }}$ rows of the corresponding $\{F\}$ vector are given by:

$$
\begin{gathered}
\{F\}_{l}=-\frac{m_{s 2}^{j}+m_{s 3}^{j}}{m_{s 2}^{j}} \frac{1}{r_{j}} \frac{\phi_{r}^{j+1}-\phi_{r}^{j-1}}{2 \Delta r}-\left[\frac{1}{r_{j}^{2}} \frac{m_{s 1}^{j}}{m_{s 2}^{j}}+\frac{1}{r_{j}} \frac{1}{m_{s 2}^{j}} \frac{m_{s 2}^{j+1}-m_{s 2}^{j-1}}{2 \Delta r}\right] \phi_{r}^{j} \\
\{F\}_{1}=-\frac{1}{\Delta r^{2}}+\frac{1}{m_{s 2}^{(2)}} \frac{m_{s 2}^{(3)}-m_{s 2}^{(1)}}{2 \Delta r} \frac{1}{2 \Delta r}-\frac{m_{s 2}^{(2)}+m_{s 3}^{(2)}}{m_{s 2}^{(2)}} \frac{1}{r_{2}} \frac{\phi_{r}^{(3)}-1}{2 \Delta r} \\
-\left[\frac{1}{r_{2}^{2}} \frac{m_{s 1}^{(2)}}{m_{s 2}^{(2)}}+\frac{1}{r_{2}} \frac{1}{m_{s 2}^{(2)}} \frac{m_{s 2}^{(3)}-m_{s 2}^{(3)}}{2 \Delta r}\right] \phi_{r}^{(2)} \\
\{F\}_{m-2}=-\frac{m_{s 2}^{m-1}+m_{s 3}^{m-1}}{m_{s 2}^{m-1}} \frac{1}{r_{m-1}} \frac{-\phi_{r}^{m-2}}{2 \Delta r}-\left[\frac{1}{r_{m-1}^{2}} \frac{m_{s 1}^{m-1}}{m_{s 2}^{m-1}}+\frac{1}{r_{m-1}} \frac{1}{m_{s 2}^{m-1}} \frac{m_{s 2}^{m}-m_{s 2}^{m-2}}{2 \Delta r}\right] \phi_{r}^{m-1}
\end{gathered}
$$

The functions $\phi_{r}$ or $\phi_{\theta}$ are solved using an iterative process similar to that described in chapter 3. Using an initial estimate of $\phi_{r}^{j},\{F\}$ is calculated using equations (4-45) through (447), and $\phi_{\theta}^{j}$ are determined by solving equation (4-35) ([K] is formed using equations (4-42) through (4-44)). Using the calculated $\phi_{\theta}^{j}$ as input, $\{F\}$ is calculated (from equations (4-39) through (4-41)) to obtain $\phi_{r}^{j}$ ([K] for this case is formed using equations (4-36) through (4-38)). Iterations are performed with the newly obtained $\phi_{r}^{j}$ to calculate $\phi_{\theta}^{j}$ and so on until convergence is reached (we call the iterations between $\phi_{r}$ and $\phi_{\theta}$ the $\phi$-iterations). The criteria used for convergence is $\frac{1}{m} \sum_{j=1}^{m}\left|\phi_{r}^{j_{\text {previous }}}-\phi_{r}^{j_{\text {current }}}\right| \leq 10^{-8}$ and $\frac{1}{m} \sum_{j=1}^{m}\left|\phi_{\theta}^{j_{\text {previous }}}-\phi_{\theta}^{j_{\text {current }}}\right| \leq 10^{-8}$ where $m$ is the total number of nodes. A less stringent convergence criterion, with a maximum of $10^{-5}$, can be 
used, but then we have to ensure (by varying the criterion between $10^{-5}$ and $10^{-8}$ ) that accurate values of $\phi_{r}^{j}$ and $\phi_{\theta}^{j}$ are obtained for the chosen criterion.

\subsubsection{Pile Deflection}

Finally, we consider the variation of $w$. We again refer back to equation (4-24b) and collect all the terms associated with $\delta w$ and $\delta\left(\frac{d w}{d z}\right)$, and equate their sum to zero:

$$
\begin{aligned}
& \int_{0}^{L_{p}} E_{p} I_{p} \frac{d^{2} w}{d z^{2}} \delta\left(\frac{d^{2} w}{d z^{2}}\right) d z+\int_{0}^{\infty} \int_{r_{p}}^{\infty} \int_{0}^{2 \pi}\left[\left(\lambda_{s}+2 G_{s}\right) w \delta w\left(\frac{d \phi_{r}}{d r}\right)^{2} \cos ^{2} \theta\right. \\
& +2 \lambda_{s} w \delta w \cos ^{2} \theta \frac{1}{r} \frac{d \phi_{r}}{d r}\left(\phi_{r}-\phi_{\theta}\right)+\left(\lambda_{s}+2 G_{s}\right) w \delta w \cos ^{2} \theta \frac{1}{r^{2}}\left(\phi_{r}-\phi_{\theta}\right)^{2} \\
& +G_{s} w \delta w \sin ^{2} \theta \frac{1}{r^{2}}\left(\phi_{r}-\phi_{\theta}\right)^{2}+2 G_{s} w \delta w \sin ^{2} \theta \frac{1}{r} \frac{d \phi_{\theta}}{d r}\left(\phi_{r}-\phi_{\theta}\right) \\
& +G_{s} w \delta w \sin ^{2} \theta\left(\frac{d \phi_{\theta}}{d r}\right)^{2}+G_{s} \frac{d w}{d z} \delta\left(\frac{d w}{d z}\right) \phi_{r}^{2} \cos ^{2} \theta \\
& \left.+G_{s} \frac{d w}{d z} \delta\left(\frac{d w}{d z}\right) \phi_{\theta}^{2} \sin ^{2} \theta\right] r d \theta d r d z+\pi r_{p}^{2} G_{s} \int_{L_{p}}^{\infty} \frac{d w}{d z} \delta\left(\frac{d w}{d z}\right) d z \\
& -\left.F_{a} \delta w\right|_{z=0}+\left.M_{a} \delta\left(\frac{d w}{d z}\right)\right|_{z=0}=0
\end{aligned}
$$

Considering a layered soil deposit as the one shown in Figure 3-1, equation (4-48a) can be simplified as: 


$$
\begin{aligned}
\int_{0}^{H_{1}} & {\left[E_{p} I_{p} \frac{d^{4} w_{1}}{d z^{4}}-2 t_{1} \frac{d^{2} w_{1}}{d z^{2}}+k_{1} w_{1}\right] \delta w_{1} d z+\int_{H_{1}}^{H_{2}}\left[E_{p} I_{p} \frac{d^{4} w_{2}}{d z^{4}}-2 t_{2} \frac{d^{2} w_{2}}{d z^{2}}+k_{2} w_{2}\right] \delta w_{2} d z } \\
+ & +\int_{H_{n-1}}^{L_{p}}\left[E_{p} I_{p} \frac{d^{4} w_{n}}{d z^{4}}-2 t_{i} \frac{d^{2} w_{n}}{d z^{2}}+k_{i} w_{n}\right] \delta w_{n} d z+\int_{L_{p}}^{\infty}\left[-2 t_{n+1} \frac{d^{2} w_{n+1}}{d z^{2}}+k_{n} w_{n+1}\right] \delta w_{n+1} d z \\
+ & {\left.\left[E_{p} I_{p} \frac{d^{3} w_{1}}{d z^{3}}-2 t_{1} \frac{d w_{1}}{d z}-F_{a}\right] \delta w_{1}\right|_{z=0}-\left.\left[E_{p} I_{p} \frac{d^{2} w_{1}}{d z^{2}}-M_{a}\right] \delta\left(\frac{d w_{1}}{d z}\right)\right|_{z=0} } \\
+ & {\left[-\left.\left\{E_{p} I_{p} \frac{d^{3} w_{1}}{d z^{3}}-2 t_{1} \frac{d w_{1}}{d z}\right\} \delta w_{1}\right|_{z=H_{1}}+\left.\left\{E_{p} I_{p} \frac{d^{3} w_{2}}{d z^{3}}-2 t_{2} \frac{d w_{2}}{d z}\right\} \delta w_{2}\right|_{z=H_{1}}\right] } \\
& +\left[\left.E_{p} I_{p} \frac{d^{2} w_{1}}{d z^{2}} \delta\left(\frac{d w_{1}}{d z}\right)\right|_{z=H_{1}}-\left.E_{p} I_{p} \frac{d^{2} w_{2}}{d z^{2}} \delta\left(\frac{d w_{2}}{d z}\right)\right|_{z=H_{1}}\right] \\
& +\ldots+\left[-\left.\left\{E_{p} I_{p} \frac{d^{3} w_{n}}{d z^{3}}-2 t_{n} \frac{d w_{n}}{d z}\right\} \delta w_{n}\right|_{z=L_{p}}-\left.2 t_{n+1} \frac{d w_{n+1}}{d z} \delta w_{n+1}\right|_{z=L_{p}}\right] \\
& +\left.E_{p} I_{p} \frac{d^{2} w_{n}}{d z^{2}} \delta\left(\frac{d w_{n}}{d z}\right)\right|_{z=L_{p}}+\left.2 t_{n+1} \frac{d w_{n+1}}{d z} \delta w_{n+1}\right|_{z=\infty}=0
\end{aligned}
$$

As before, the $n^{\text {th }}$ (bottom) layer is split into two parts, with the part below the pile denoted by the subscript $n+1$; therefore, in the above equation, $H_{n}=L_{p}$ and $H_{n+1} \rightarrow \infty$. The soil parameters in the above equation are defined as:

$$
\begin{gathered}
t_{i}=\left\{\begin{array}{c}
\frac{1}{2} \int_{r_{p}}^{\infty} \int_{0}^{2 \pi} G_{s i}\left(\phi_{r}^{2} \cos ^{2} \theta+\phi_{\theta}^{2} \sin ^{2} \theta\right) r d \theta d r ; i=1,2, \ldots, n \\
\frac{1}{2} \int_{0}^{\infty} \int_{0}^{2 \pi} G_{s n}\left(\phi_{r}^{2} \cos ^{2} \theta+\phi_{\theta}^{2} \sin ^{2} \theta\right) r d \theta d r ; \quad i=n+1
\end{array}\right. \\
k_{i}=\int_{r_{p}}^{\infty} \int_{0}^{2 \pi}\left[\left(\lambda_{s i}+2 G_{s i}\right)\left(\frac{d \phi_{r}}{d r}\right)^{2} \cos ^{2} \theta+2 \lambda_{s i} \frac{1}{r} \frac{d \phi_{r}}{d r}\left(\phi_{r}-\phi_{\theta}\right) \cos ^{2} \theta\right. \\
+\left(\lambda_{s i}+2 G_{s i}\right) \frac{1}{r^{2}}\left(\phi_{r}-\phi_{\theta}\right)^{2} \cos ^{2} \theta+G_{s i} \frac{1}{r^{2}}\left(\phi_{r}-\phi_{\theta}\right)^{2} \sin ^{2} \theta \\
\left.+2 G_{s i} \frac{1}{r}\left(\phi_{r}-\phi_{\theta}\right) \frac{d \phi_{\theta}}{d r} \sin ^{2} \theta+G_{s i}\left(\frac{d \phi_{\theta}}{d r}\right)^{2} \sin ^{2} \theta\right] r d \theta d r
\end{gathered}
$$

From equation (4-48b), we get the same set of equations of pile deflection, as given in chapter 3, for the domains $L_{p} \leq z<\infty$ and $0 \leq z \leq L_{p}$, respectively. The parameters $t_{i}$ and $k_{i}$ of 
equations (4-49) and (4-50) should be used with those equations. The equations involving $w(z)$ are normalized in a similar way as done in chapters 2 and 3; the normalized parameters to be used with the normalized equations are given by:

$$
\begin{gathered}
\tilde{t}_{i}=\left\{\begin{array}{c}
\frac{L_{p}^{2}}{2 E_{p} I_{p}} \int_{r_{p}}^{\infty} \int_{0}^{2 \pi} G_{s i}\left(\phi_{r}^{2} \cos ^{2} \theta+\phi_{\theta}^{2} \sin ^{2} \theta\right) r d \theta d r ; i=1,2, \ldots, n \\
\frac{L_{p}^{2}}{2 E_{p} I_{p}} \int_{0}^{\infty} \int_{0}^{2 \pi} G_{s n}\left(\phi_{r}^{2} \cos ^{2} \theta+\phi_{\theta}^{2} \sin ^{2} \theta\right) r d \theta d r ; \quad i=n+1
\end{array}\right. \\
\tilde{k}_{i}=\frac{L_{p}^{4}}{E_{p} I_{p}} \int_{r_{p}}^{\infty} \int_{0}^{2 \pi}\left[\left(\lambda_{s i}+2 G_{s i}\right)\left(\frac{d \phi_{r}}{d r}\right)^{2} \cos ^{2} \theta+2 \lambda_{s i} \frac{1}{r} \frac{d \phi_{r}}{d r}\left(\phi_{r}-\phi_{\theta}\right) \cos ^{2} \theta\right. \\
+\left(\lambda_{s i}+2 G_{s i}\right) \frac{1}{r^{2}}\left(\phi_{r}-\phi_{\theta}\right)^{2} \cos ^{2} \theta+G_{s i} \frac{1}{r^{2}}\left(\phi_{r}-\phi_{\theta}\right)^{2} \sin ^{2} \theta \\
\left.+2 G_{s i} \frac{1}{r}\left(\phi_{r}-\phi_{\theta}\right) \frac{d \phi_{\theta}}{d r} \sin ^{2} \theta+G_{s i}\left(\frac{d \phi_{\theta}}{d r}\right)^{2} \sin ^{2} \theta\right] r d \theta d r ; i=1,2, \ldots, n
\end{gathered}
$$

Numerical integrations along $r$ and $\theta$ are required for the calculation of $\tilde{k}_{i}$ and $\tilde{t}_{i}$. A threedimensional discretization of the soil mass along the $r, \theta$, and $z$ directions is done with $\Delta r, \Delta \theta$ and $\Delta \tilde{z}$ as the step lengths, respectively (Figure 4-6). Note that the step length $\Delta r$ used for calculating $\tilde{k}_{i}$ and $\tilde{t}_{i}$ is the same as that used in the finite difference discretization used for calculating $\phi_{r}$ and $\phi_{\theta}$. Therefore, the finite difference discretization (along the radial direction) coincides with this three-dimensional discretization.

At any given depth $\tilde{z}_{l}$ (the subscript $l$ is used to denote the nodes along the $z$ direction), integration following the trapezoidal rule is performed first along $\theta$ for any radial distance $r_{j}$ with a step length of $r_{j} \Delta \theta$. The value of the integral thus obtained is used as the integrand at the $j^{\text {th }}$ node in the radial direction, for subsequent integration along $r$ with $\Delta r$ as the step length. The sequential integrations, first along $\theta$ and then along $r$, gives $\tilde{k}_{i}$ and $\tilde{t}_{i}$ at any particular node $l$ at depth $\tilde{z}_{l}$. Values of $\tilde{k}_{i}$ and $\tilde{t}_{i}$ are calculated for each depth $\tilde{z}_{l}$ within each layer $i$ and then the average values of $\tilde{k}_{i}$ and $\tilde{t}_{i}$ are determined for each of the layers, which are used for the calculation of pile deflection. In order to perform the integration, the correct values of elastic constants are determined at each node (corresponding to the level of stress or strain at the nodes) by an iterative nonlinear algorithm described later. 


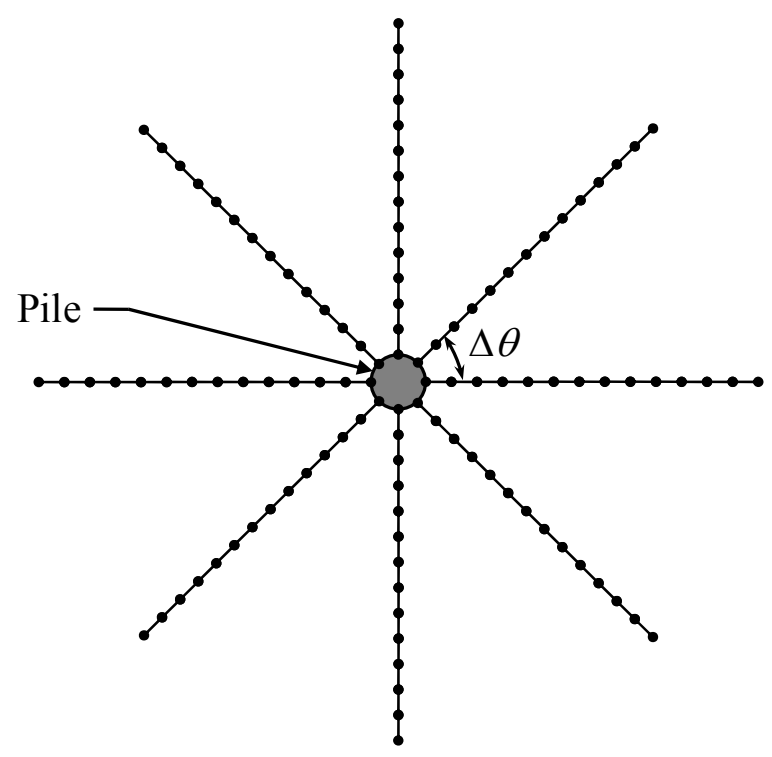

Top View

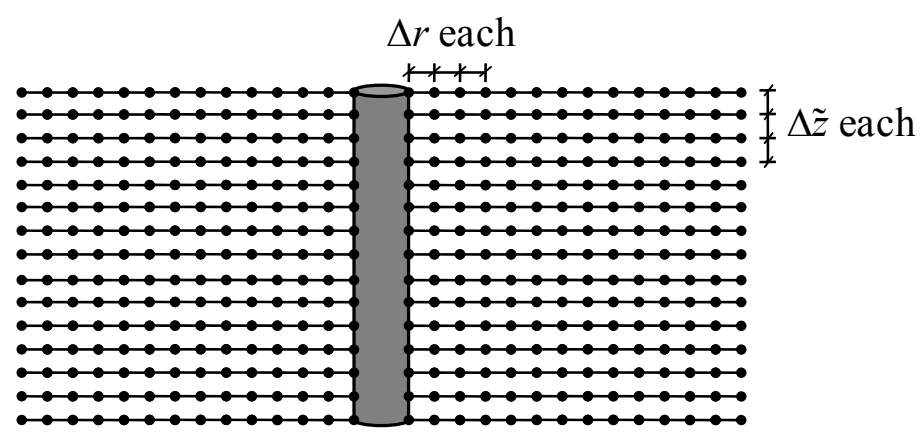

Front View

Figure 4-6 Discretization in a Soil Mass

Solution of pile deflection can be obtained analytically if we assume that the parameters $\tilde{k}_{i}$ and $\tilde{t}_{i}$ are constants. The secant modulus approach used in the nonlinear algorithm, described later, makes the parameters $\tilde{k}_{i}$ and $\tilde{t}_{i}$ constant at every instance of loading. Hence, the method of initial parameters, described in chapter 2 , can be used recurrently to obtain the nonlinear pile deflection, slope, bending moment and shear force as functions of applied load. 


\subsection{Interdependence and Iterative Solutions of Pile and Soil Displacements}

It is evident from equations (4-51) and (4-52) that the functions $\phi_{r}(r)$ and $\phi_{\theta}(r)$ need to be known to estimate the parameters $\tilde{k}_{i}$ and $\tilde{t}_{i}$. This means that pile deflection cannot be obtained unless $\phi_{r}(r)$ and $\phi_{\theta}(r)$ are determined. In order to determine $\phi_{r}(r)$ and $\phi_{\theta}(r)$, the quantities $m_{s 1}$, $m_{s 2}, m_{s 3}, n_{s 1}$ and $n_{s 2}$ need to be known, which, in turn, depend on the pile deflection $w$ and slope $d w / d z$. Therefore, the pile-deflection and the soil-displacement equations are coupled (similarly to the linear elastic case of chapter 3), and have to be solved simultaneously using an iterative scheme (note that this iteration is separate from the $\phi$-iterations described before).

In order to solve the coupled equations, trial profiles for $\phi_{r}$ and $\phi_{\theta}$ are assumed and the values of $\tilde{t}_{i}$ and $\tilde{k}_{i}$ are obtained from equations (4-51) and (4-52) using numerical integration. Pile deflection is then obtained using the method of initial parameters so that $m_{s 1}, m_{s 2}, m_{s 3}, n_{s 1}$ and $n_{s 2}$ can be determined. Using the calculated values of $m_{s 1}, m_{s 2}, m_{s 3}, n_{s 1}$ and $n_{s 2}, \phi_{r}$ and $\phi_{\theta}$ are determined by solving equations (4-33) and (4-34) (using $\phi$-iterations). The newly obtained profiles of $\phi_{r}$ and $\phi_{\theta}$ are then compared with the previous (trial) profiles. If the differences are

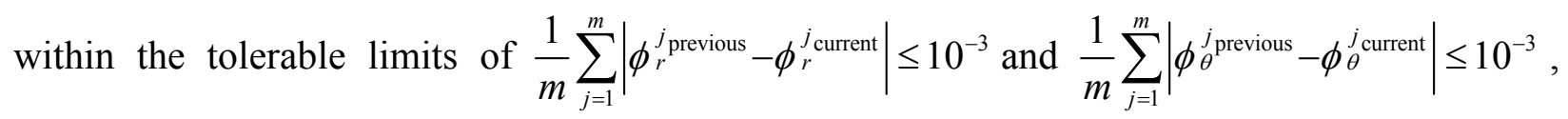
then we accept the $\phi_{r}$ and $\phi_{\theta}$, and the corresponding $w$ as the final solutions (note that the convergence criterion of $10^{-3}$ is different from the convergence criterion of $10^{-8}$ for the $\phi$ iterations described before) . However, if the differences are greater than the tolerable limits, then we assume the newly obtained profiles of $\phi_{r}$ and $\phi_{\theta}$ as the new trial profiles for the next iteration and repeat the process until convergence on both $\phi_{r}$ and $\phi_{\theta}$ is achieved (we call this set of iterations involving $w, \phi_{r}$ and $\phi_{\theta}$ the $w$-iterations). The convergence criterion for the $\phi$ iterations is more stringent than that for the $w$-iterations because the $\phi$-iterations are at the core of this nonlinear analysis, and the accuracy of $w$, obtained from the $w$-iterations, depends on the accuracy of $\phi_{r}$ and $\phi_{\theta}$ obtained from the $\phi$-iterations.

\subsection{Nonlinear Algorithm}

The nonlinear algorithm is fundamentally based on the concept of applying the external load (or/and moment) (or a fraction thereof) and solve for pile deflection (through $\phi$-iterations 
and $w$-iterations) after obtaining the correct values of soil modulus based on the correct displacement, strain and the stress fields, which are consistent with the applied loads. Incremental load application is not necessary in this algorithm because the analysis is based on secant modulus (ratio of total accumulated stress to corresponding strain) approach. If, for example, the design load acting on the pile is $F_{a}=300 \mathrm{kN}$ and $M_{a}=100 \mathrm{kNm}$, the total load of $300 \mathrm{kN}$ and $100 \mathrm{kNm}$ can be applied in one step and pile deflection can be obtained. However, if one needs to follow the progression of displacements as a function of the applied load (e.g., a plot of head deflection versus applied load), then fractions of the total design load have to be applied recurrently in increasing magnitudes (e.g., in the sequence of, say, $30 \mathrm{kN}, 10 \mathrm{kNm}$; 60 $\mathrm{kN}, 20 \mathrm{kNm}$ and so on) and the corresponding deflections have to be determined.

In order to start the algorithm, a small magnitude (say, $1 \mathrm{kN}$ ) of force (and/or moment) is applied to determine the initial profiles of $\phi_{r}$ and $\phi_{\theta}$. For this "initial" load application, it is assumed that the soil mass is homogeneous, isotropic and elastic within each layer. Thus, the initial applied load has to be sufficiently small in order for the modulus to not degrade by any significant amount such that the assumption of homogeneity with respect to soil modulus holds good. For such an assumption, the analysis and algorithm developed in chapter 3 are valid, and are used to obtain the initial pile deflection. For calculating the values of $k_{i}$ and $t_{i}$ for the initial load application, the initial values of the elastic constants $\left(G_{s 0}\right.$ and $v_{s 0}$; or $G_{s 0}$ and $K_{s 0}$; or $\lambda_{s 0}$ and $G_{s 0}$; or $E_{s 0}$ and $v_{s 0}$ ) are used. At the end of the initial load application, the profiles of $\phi_{r}$ and $\phi_{\theta}$ obtained from the initial load step are recorded for use as the initial trial profiles $\left(\phi_{r}{ }^{\text {ini }}\right.$ and $\left.\phi_{\theta}{ }^{\text {ini }}\right)$ during the actual load application.

Next, the actual design load (or a fraction of it) is applied. Profiles of $\phi_{r}{ }^{\text {ini }}$ and $\phi_{\theta}{ }^{\text {ini }}$ are used to calculate the strains using equation (3-3) at different points within the soil mass (the values of pile deflection $w_{i}$ and slope $d w_{i} / d z$ obtained from the initial load application are used in the calculation). Strains are calculated at the discrete points spaced at intervals of $\Delta r$ and of $\Delta \theta$ along the radial and circumferential directions, respectively (Figure 4-6). This discretization is the same as described before for the calculation of $\tilde{k}_{i}$ and $\tilde{t}_{i}$. Using the values of strains, the elastic constants are calculated (by the use of an appropriate stress-strain relationship) at each of the discretized points. From the calculated values of elastic constants and $\phi_{r}{ }^{\text {ini }}$ and $\phi_{\theta}{ }^{\text {ini }}$ at the 
nodes, $\tilde{t}_{i}$ and $\tilde{k}_{i}$ are calculated using equations (4-51) and (4-52) (by the use of numerical integration).

After the calculation of $\tilde{k}_{i}$ and $\tilde{t}_{i}$, profiles of pile deflection and slope are obtained using the method of initial parameters. Using these values, $m_{s 1}, m_{s 2}, m_{s 3}, n_{s 1}$ and $n_{s 2}$ are then calculated from equations (4-26) through (4-29) and (4-32), respectively, using numerical integrations. Here, the numerical integration (following the trapezoidal rule) is done along $\theta$ and $\tilde{z}$. For any radial distance $r_{j}$, integration along $\theta$ is first performed (with a step length of $r_{j} \Delta \theta$ ). The value of the integral thus obtained is then used as the integrand to perform the integration along $\tilde{z}$ with a step length of $\Delta \tilde{z}$.

After obtaining $m_{s 1}, m_{s 2}, m_{s 3}, n_{s 1}$ and $n_{s 2}, \phi_{r}$ and $\phi_{\theta}$ are determined by solving equations (4-30b) and (4-31) using $\phi$-iterations. Using the new values of $\phi_{r}, \phi_{\theta}, w_{i}$ and $d w_{i} / d z$, strains and subsequently soil moduli are recalculated to again calculate $k_{i}$ and $t_{i}$, which are then used to calculate pile deflection. Iterations ( $w$-iterations) are performed until the profiles of $\phi_{r}$ and $\phi_{\theta}$ between successive $w$-iterations match to the specified tolerable limits. Tolerance limits of $\frac{1}{m} \sum_{j=1}^{m}\left|\phi_{r}^{j_{\text {previous }}}-\phi_{r}^{j_{\text {current }}}\right| \leq 10^{-3}$ and $\frac{1}{m} \sum_{j=1}^{m}\left|\phi_{\theta}^{j_{\text {previous }}}-\phi_{\theta}^{j_{\text {current }}}\right| \leq 10^{-3}$ are used, which ensured proper convergence. After the convergence is ensured, we get the pile deflection corresponding to the applied load.

As mentioned before, the above solution scheme is valid for the full design load or a fraction of it. If fractions of the design load are recurrently applied in increasing magnitudes to determine how the pile deflection profile evolves with time, the same procedure has to be repeated for each such load application. The nonlinear algorithm is outlined in Figure 4-7. 


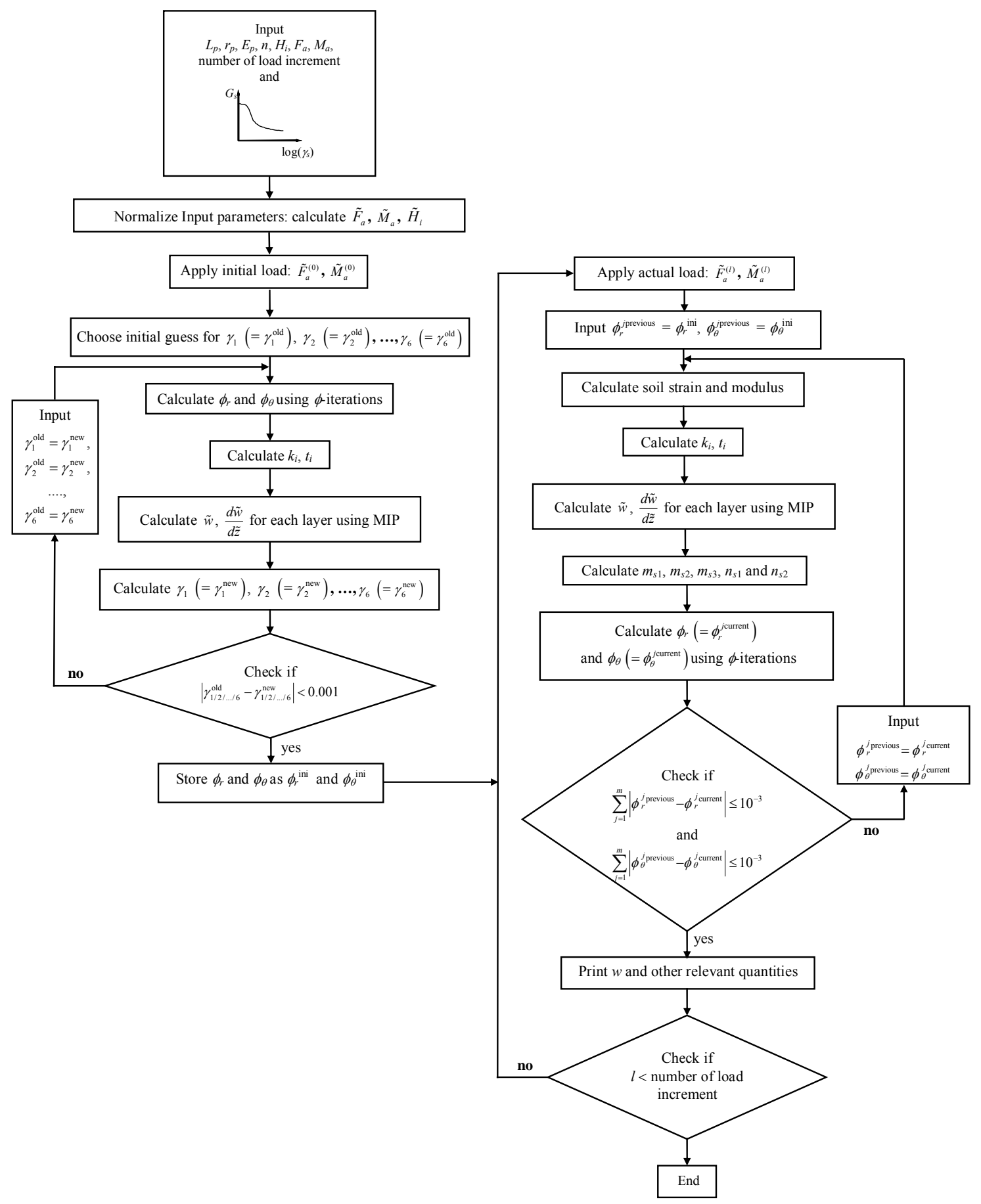

Figure 4-7 Nonlinear Solution Flow Chart 


\subsection{Results}

In this section, we consider two examples to illustrate how the nonlinear analysis works. In the first example, we consider a 20 -m long concrete pile $\left(E_{p}=25 \mathrm{GPa}\right)$ with a diameter of 0.6 $\mathrm{m}$ embedded in a layered sand deposit (the water table is assumed to be at a large depth). The top $5 \mathrm{~m}$ of the deposit consists of a sand with a relative density $\mathrm{D}_{R}=50 \%\left(\mathrm{D}_{R}\right.$ describes the closeness of packing of sand grains and is defined as the ratio $\left(e_{\max }-e\right) /\left(e_{\max }-e_{\min }\right)$, where $e$ is the void ratio of sand at its natural state, $e_{\max }$ is the void ratio when the sand is reconstituted in a manner such that it is in its loosest state, and $e_{\min }$ is the void ratio when the sand is reconstituted in a manner such that it is in its densest state), the second layer extends from a depth of $5 \mathrm{~m}$ to a depth of $10 \mathrm{~m}$ (5-m thickness) and has a $D_{R}=60 \%$, while the third and bottom layer has a relative density of $70 \%$ and extends from a depth of $10 \mathrm{~m}$ to large distance downward. We use the $f$ - $g$ model of modulus degradation for our nonlinear analysis. We assume that, for all the three sand layers, the critical state friction angle $\phi_{c}=33^{\circ}$, the unit weight is equal to $18 \mathrm{kN} / \mathrm{m}^{3}$ and the coefficient of earth pressure is 0.45 . We used standard correlation of Bolton (1986) to obtain the peak friction angle $\phi_{p}$ for the three layers as $40.5^{\circ}, 40^{\circ}$ and $39.5^{\circ}$ (for layers 1,2 and 3 , respectively). We used these values as input (with $c=0$ ) in equation (4-7) for use with the $f-g$ model. We further assumed $e_{\max }=0.8$ and $e_{\min }=0.4$ and used the relationship of $\mathrm{D}_{\mathrm{R}}$ to obtain the initial void ratios $e_{0}$ as $0.6,0.56$ and 0.52 for layers 1,2 and 3 , respectively. We calculated the initial stiffness modulus using equation (4-20a). We used $f=0.97$ and $g=0.23$ for all the layers. We assume that the pile head and base are free to translate and rotate. In our nonlinear analysis, we divide each sand layer into a number of sub-layers so that degradation of the moduli can be calculated in an accurate way. We also performed p-y analysis for the same problem using the software PYGMY (Stewart 2000) using similar discretization of the pile. Figure 4-8 shows the head deflection as a function of applied force. It is evident that our analysis and $p-y$ method produces pile deflections that are of the same order of magnitude. 


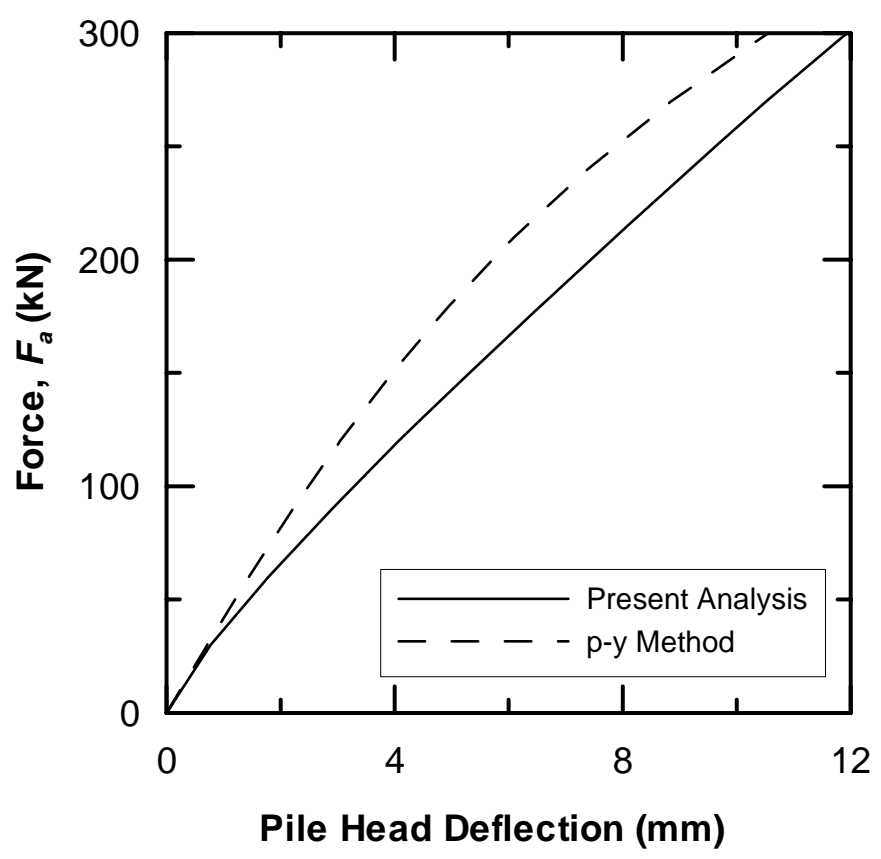

Figure 4-8 Head Deflection as a Function of Applied Force for a Pile in Sand

Next, we consider a problem of a $15-\mathrm{m}$ long concrete pile $\left(E_{p}=25 \mathrm{GPa}\right)$ embedded in a bed of clay underlain by sand. The water table is at the ground surface. The diameter of the pile is $1.0 \mathrm{~m}$ and is free at both the head and base. A horizontal force acts at the head. The clay deposit is assumed to be normally consolidated (with a coefficient of earth pressure of 0.45) extending from the ground surface to a depth of $13 \mathrm{~m}$. We assumed that the bulk unit weight of clay is $16 \mathrm{kN} / \mathrm{m}^{3}$ and $\phi_{c}=30^{\circ}$ for both the layers. The sand layer, in which the pile base sits, consists of sand with $\mathrm{D}_{\mathrm{R}}=70 \%, \phi_{c}=33^{\circ}, e_{\max }=0.8$ and $e_{\min }=0.4$, for which we got $\phi_{p}=40.5^{\circ}$ and $e_{0}=0.52$. We used equation (4-20b) to calculate the initial shear modulus of clays and used equation (4-20a) for sand. For a comparison with the p-y method, we considered the criteria for "soft-clay" as prescribed by API (1993). For obtaining the initial shear modulus of the clay layers, we assumed the values available for lower Cromer till, which belongs to the category of soft clays (Chakraborty, T. and Salgado, R., personal communication) for inputs in equation (420b): $C_{g}=150.0, n_{g}=0.3, e_{g}=2.17$. In order to calculate the initial void ratio of clay $e_{0}$ from the existing mean effective stress $\sigma_{m}$ and OCR, we used the following equation

$$
e_{0}=N-\lambda \ln \left(\frac{\sigma_{m}}{P_{a}}\right)-\kappa \ln \left(\frac{1}{\mathrm{OCR}}\right)
$$


where $N=0.47, \lambda=0.063$ and $\kappa=0.009$. Using the above equation, $e_{0}$ for clay was found to be $0.65,0.58,0.55$ and 0.44 for the soil in the depth ranges $0 \mathrm{~m}$ to $3 \mathrm{~m}, 3 \mathrm{~m}$ to $6 \mathrm{~m}, 6 \mathrm{~m}$ to $9 \mathrm{~m}$, and $9 \mathrm{~m}$ to $13 \mathrm{~m}$, respectively. The undrained shear strength (cohesion) of clay $s_{u}$ was calculated using the following equation:

$$
\frac{s_{u}}{\sigma_{v}}=\left(\frac{\phi_{c}}{100}\right)(\mathrm{OCR})^{0.8}
$$

where $\sigma_{v}$ is the vertical effective stress at a depth. The values of $s_{u}$ obtained for the clay layers are $2.79 \mathrm{kPa}, 8.34 \mathrm{kPa}, 13.9 \mathrm{kPa}$ and $19.5 \mathrm{kPa}$ for the depth ranges of $0 \mathrm{~m}$ to $3 \mathrm{~m}, 3 \mathrm{~m}$ to $6 \mathrm{~m}, 6$ $\mathrm{m}$ to $9 \mathrm{~m}$ and $9 \mathrm{~m}$ to $15 \mathrm{~m}$, respectively. The value of $s_{u}$ was given as input to the p-y analysis (PYGMY) and also to equation (4-7) for use with the $f$ - $g$ model. We assumed $f=1$ and $g=0.3$ for clay in the analysis. Figure 4-9 shows the head deflection as a function of applied horizontal force for our method and the p-y method. It is again evident that our analysis and p-y method produced results that are of the same order of magnitude.

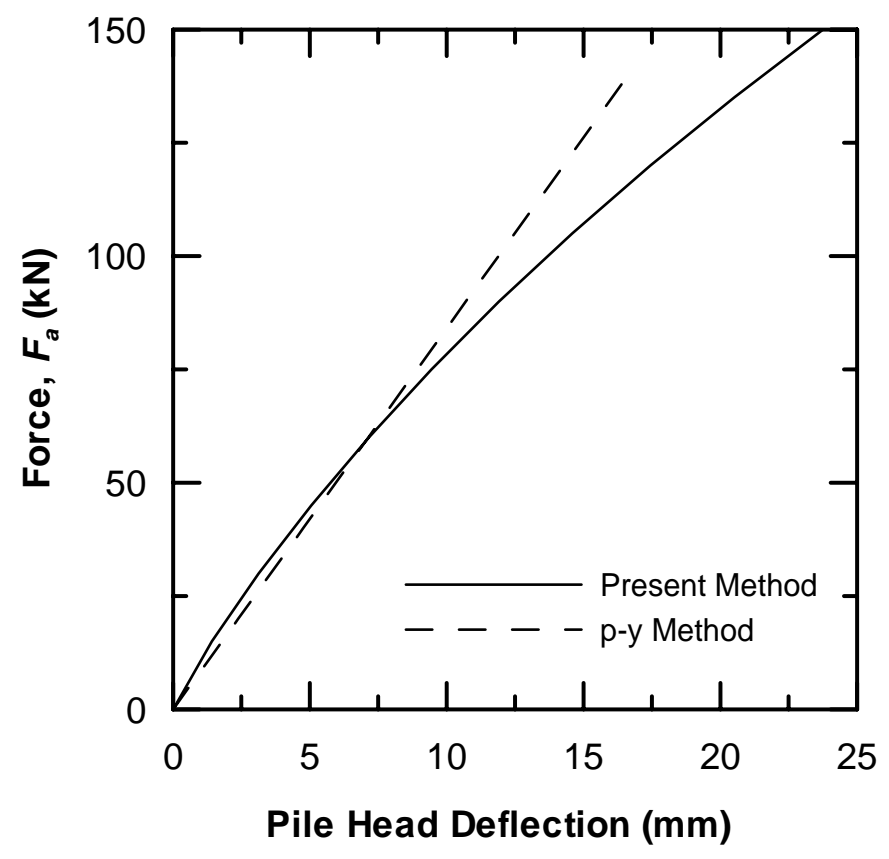

Figure 4-9 Head Deflection as a Function of Applied Force for a Pile in Sand 
Finally, we consider the pile load test performed at the Orange County in Indiana. A closed-ended steel pipe pile of diameter $356 \mathrm{~mm}$ and thickness $12.7 \mathrm{~mm}$ was embedded into the ground to a depth of $17.4 \mathrm{~m}$. The soil profile is described in Figure 4-10. The soil properties are given in Table 4-1.

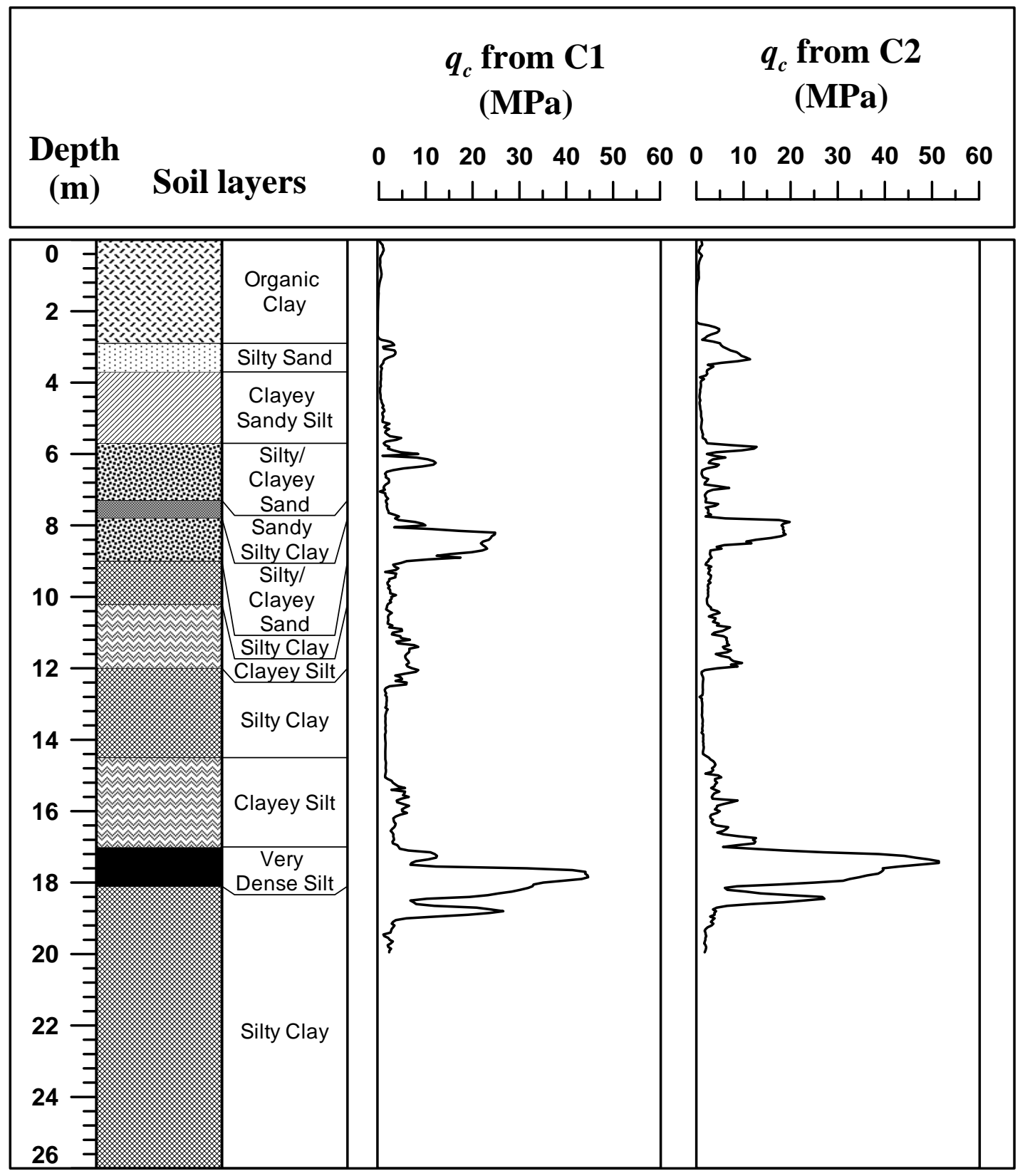

Figure 4-10 Soil Profile at the Pile Load Test Site in Orange County, Indiana 
Table 4-1 Soil Properties at the Pile Load Test Site in Orange County, Indiana

\begin{tabular}{|c|c|c|c|c|c|c|c|c|c|c|c|c|c|c|c|}
\hline $\begin{array}{l}\text { Layer } \\
\text { No. }\end{array}$ & $\begin{array}{l}\text { Depth } \\
(\mathrm{m})\end{array}$ & $\begin{array}{l}\mathrm{W}_{\mathrm{c}} \\
(\%)\end{array}$ & $\begin{array}{l}\text { LL } 1 \\
(\%)(\end{array}$ & & $\begin{array}{l}\text { PI } \\
(\%)\end{array}$ & $\begin{array}{l}\text { Total Unit Weight } \\
\qquad\left(\mathrm{kN} / \mathrm{m}^{3}\right)\end{array}$ & $\begin{array}{l}D_{R} \\
(\%)\end{array}$ & $e_{0}$ & $C_{c}$ & $C_{s}$ & $\begin{array}{c}c_{v} \\
\left.\mathrm{~m}^{2} / \mathrm{sec}\right)(\end{array}$ & $\begin{array}{c}\sigma_{v p}^{\prime} \\
(\mathrm{kPa})\end{array}$ & OCR & $\begin{array}{c}\phi_{c} \\
(\operatorname{deg})(\end{array}$ & $\begin{array}{c}S_{u} \\
(\mathrm{kPa})\end{array}$ \\
\hline 1 & $0-2.9$ & 96 & 138 & 49 & 89 & 13.4 & - & 3.56 & 0.67 & 0.821 & $.66 \mathrm{E}-06$ & - & - & - & - \\
\hline 2 & $2.9-3.7$ & 15 & - & - & - & $22^{*}$ & 78 & - & & - & - & - & - & $31^{*}$ & - \\
\hline 3 & $3.7-5.7$ & 19 & 18 & 10 & 8 & 21.6 & - & 0.47 & 0.11 & 0.032 & $.45 \mathrm{E}-07$ & 265 & 5.6 & - & 78 \\
\hline 4 & $5.7-7.3$ & - & - & - & - & $22^{*}$ & 52 & - & - & - & - & - & - & $29^{*}$ & - \\
\hline 5 & $7.3-7.8$ & - & - & - & - & $21^{*}$ & - & - & - & - & - & - & - & - & - \\
\hline 6 & $7.8-9.0$ & - & - & - & - & $22^{*}$ & 81 & - & - & - & - & - & - & $29^{*}$ & - \\
\hline 7 & $9.0-10.2$ & 25 & 37 & 18 & 19 & 20.1 & - & 0.73 & 0.19 & 0.036 & $.82 \mathrm{E}-07$ & 365 & 3.2 & - & 220 \\
\hline 8 & $10.2-12.0$ & 023 & 29 & 19 & 10 & 20.6 & & 0.63 & 0.13 & 0.013 & $.43 \mathrm{E}-06$ & 265 & 1.9 & - & 320 \\
\hline 9 & $12.0-14.5$ & 515 & 21 & 12 & 9 & 21.9 & - & 0.45 & 0.10 & 0.015 & $30 \mathrm{E}-07$ & 750 & 4.9 & - & 103 \\
\hline 10 & $14.5-17$ & 11 & 22 & 12 & 10 & 21.6 & - & 0.40 & 0.08 & 0.023 & $.75 \mathrm{E}-07$ & 365 & 2.0 & - & 292 \\
\hline 11 & $17-18.4$ & - & - & - & - & $21^{*}$ & $95 \sim 100$ & - & - & - & - & - & - & $30^{*}$ & - \\
\hline
\end{tabular}

Note: $\mathrm{w}_{\mathrm{c}}=$ natural water content, $e_{0}=$ initial void ratio, $\mathrm{LL}=$ liquid limit, $\mathrm{PL}=$ plastic limit, $\mathrm{PI}=$ plasticity index; $C_{c}=$ compression index; $C_{s}=$ recompression index, $c_{v}=$ vertical coefficient of cosolidation; $\sigma_{v p}^{\prime}=$ preconsolidation pressure, $\mathrm{OCR}=$ overconsolidation ratio, $\phi_{c}=$ critical state friction angle, $s_{u}=$ undrained shear strength, $D_{R}=$ relative density.

* These values are assumed.

For analysis, we assume alternate clay and sand layers (layers 1, 3, 5 and 7 are assumed to be clay while layers 2, 4 and 6 are assumed to be sands). The seventh layer is assumed to have properties that are approximately the average of the layers 7 through 10. The initial void ratio assumed for all the layers is 0.5 except for the top layer which is assumed to have a void ratio of 1.6. For clays, the parameters corresponding to those of lower Cromer till, described above, are assumed for determining the initial shear modulus. The $f$ - $g$ model with $f=0.97$ and $g$ $=0.23$ is considered. Figure 4-11 shows the plot of the pile head deflection versus applied horizontal load as obtained from the field experiment and the analysis. It is evident that the analysis result matches reasonably well with the field result. 


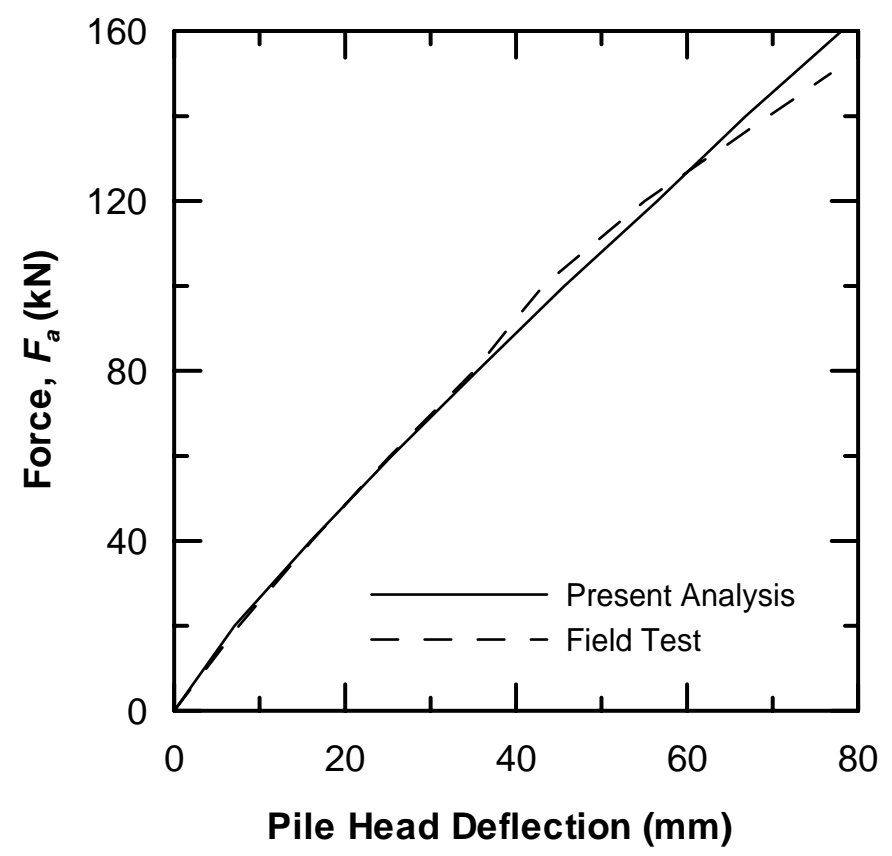

Figure 4-11 Head Deflection versus Applied Force for the Orange County Pile Load Test

\section{8. $\underline{\text { Summary }}$}

The equations for the linear elastic analysis are modified to take into account soil heterogeneity in the radial and tangential directions. The developed equations were coupled with a nonlinear algorithm that accounts for the degradation of soil modulus (which renders the soil heterogeneous) due to soil nonlinearity. A nonlinear algorithm, based on a secant modulus approach, was developed that explicitly takes into account the modulus degradation of each soil element surrounding the pile and relates it to the degraded soil resistance with increasing applied load. Thus, the three-dimensional interaction between the pile and the nonlinear soil is taken into account. Nonlinear soil constitutive relationships are outlined that can be used to obtain the nonlinear pile response. Comparisons were made with the p-y method and a field load test. The method predicts the pile deflection reasonably well. 


\section{CHAPTER 5. PILE GROUP ANALYSIS}

\subsection{Introduction}

In this chapter, the interaction of piles embedded in a linear elastic continuum is studied. The influence of one pile on any other in a group is modeled by modifying the soil resistance which is assumed to be a function of the number of piles and their positions. The additional displacement that a pile undergoes due to the loading of the other is captured.

\section{2. $\underline{\text { Overview }}$}

Interactions between piles within a pile group play an important role in determining the response of the group to external lateral loads. As a pile group is loaded, each pile pushes the soil in the direction of the applied force and generates stresses in the soil mass. Each pile creates a zone of influence within which the stresses caused by the pile are significant. Such zones of influence of all the piles in a group overlap due to the close pile spacings (Figure 1-4). The overlapping zones of influence of each pile not only transfer load to neighboring piles but also cause a reduction in the soil stiffness between piles (Matlock et al 1980, Meimon et al. 1986, Brown et al. 1987, McVay 1996).

The p-y method, modified to take into account the overall stiffness reduction of group piles, is one of the most common methods of pile group analysis (Brown et al. 1988, 1987). In this method, the soil resistance $p$ is reduced by multiplying $p$ by a factor $f$ (called the $p$ multiplier). The values of the p-multipliers are a function of spacing and position of the piles (Reese et al. 2006). The multipliers have been back-calculated to match experimental and numerical results (Brown and Shie 1991, Ruesta and Townsend 1997, Rollins et al. 1998, Zhang et al. 1999b, Ng et al. 2001, Holloway et al. 1981, Baguelin et al. 1985, McVay et al. 1994, 1995, 1998, Ilyas et al. 2004).

Alternative approaches are also available based on the concept of beams on elastic foundations. Ooi and Duncan (1994) and Ooi et al. (2004) addressed the issue of increase in 
deflection by a group-amplification procedure, which is based on the characteristic load method (Duncan et al. 1994). Davisson (1970), based on a subgrade-reaction analysis, proposed a reduction in the values of the modulus of subgrade reaction for use in group analysis. Dunnuvant and O'Neill (1986) proposed a reduction in stiffness of the piles depending on their position in the group. Nogami and Paulson (1985) used a network of Winkler springs interconnected to all the piles in the group and a transfer matrix to analyze the problem. Bogard and Matlock (1983) replaced the actual piles within a group with an equivalent single pile (applicable for closely-spaced piles) and developed equivalent p-y curves. They proposed a ymultiplier concept (with multiplier values greater than one) to account for the excess deflection of pile groups with respect to that of individual piles (applicable for groups with widely spaced piles). Ashour et al. (2001) and (2004) applied the strain wedge model to analyze pile groups.

Several continuum-based approaches are also available. Poulos (1971b) pioneered the continuum-based research by modifying his boundary integral technique, based on Mindlin's solution, to account for interaction between piles within groups. Many researchers have since then used different forms of the boundary integral technique, including the boundary element method, and have approximately accounted for soil nonlinearity and heterogeneity (Poulos 1975, Banerjee 1978, Banerjee and Davies 1980, Sharnouby and Novak 1985, Basile 1999, Xu and Poulos 2000). The finite element method, with several modifications (e.g., finite elements with Fourier technique, finite elements with substructuring, finite elements with periodic boundary conditions), has also been used quite extensively to model pile groups in elastic and elastoplastic soils (Chow 1987, Shibata et al. 1988, Kooijman 1989, Brown and Shie 1991, Bransby 1996, Zhang et al. 1999b, Wakai et al. 1999, Zhang and Small 2000, Law and Lam 2001, Budiman and Ahn 2005). That apart, the discrete element method (Holloway et al. 1981), the finite difference method (Comodromos and Pitilakis 2005) and the variational method (Shen and Teh 2002) have been used to analyze pile groups.

A few mixed methods of analysis are also available, which are the combinations of the continuum-based approach and the beam-on-foundation approach. Focht and Koch (1973) combined the integral equation approach of Poulos (1971b) and the p-y method, assuming that the deflection of a pile group depends both on the nonlinear soil behavior and on the pile-soilpile interaction. A similar hybrid model was developed by Leung and Chow (1987), who modeled the soil response using the subgrade reaction method, and interaction between piles was 
captured using Mindlin's solution. Reese et al. (1984) modified the method of Focht and Koch (1973) by calculating the elastic deflection from the linear portion of the p-y curves. O'Neill et al. (1977) proposed a mixed method involving Mindlin's solution and functional relationships between load and deformations at the pile head, which were developed using the finite difference method and cubic spline functions. Based on elastic interaction in a horizontal plane, Hariharan and Kumarasamy (1983) obtained expressions for load and displacement multipliers for p-y curves.

In this chapter, we propose coefficients $f$ (Reese et al. 2006) to be multiplied with the soil resistances $k_{i}$ and $t_{i}$ of the single-pile analysis (see chapter 4) to obtain the modified soil resistances for pile groups. Thus, the single-pile analysis is modified to obtain the nonlinear response of pile groups. We consider pile groups consisting of 2, 3, 4 and 6 piles.

\subsection{Soil Resistance for Pile Groups}

The two-pile-group problem can be described by Figure 5-1. For coefficient $f$ to be used for pile 1 and 2 are respectively given by:

$$
\begin{aligned}
& f=0.7\left(\frac{r_{12}}{2 r_{p}}\right)^{0.26} \leq 1.0 \\
& f=0.48\left(\frac{r_{12}}{2 r_{p}}\right)^{0.38} \leq 1.0
\end{aligned}
$$

where $r_{12}$ is the center-to-center spacing between the two piles.

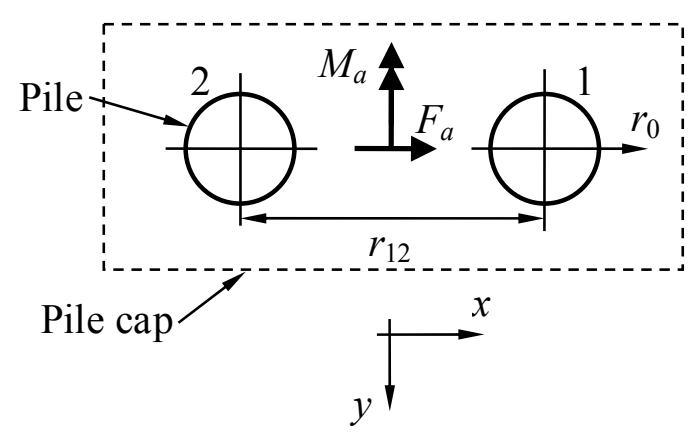

Figure 5-1 Two-Pile Group 
The three-pile group can be described by Figure 5-2. The coefficient $f$ for pile 1 can be expressed as:

$$
f=\beta_{1} \beta_{2}
$$

with

$$
\begin{aligned}
& \beta_{1}=0.7\left(\frac{r_{12}}{2 r_{p}}\right)^{0.26} \leq 1.0 \\
& \beta_{2}=0.7\left(\frac{r_{12}+r_{23}}{2 r_{p}}\right)^{0.26} \leq 1.0
\end{aligned}
$$

For pile 2, equation (5-3) is valid with

$$
\begin{aligned}
& \beta_{1}=0.7\left(\frac{r_{23}}{2 r_{p}}\right)^{0.26} \leq 1.0 \\
& \beta_{2}=0.48\left(\frac{r_{12}}{2 r_{p}}\right)^{0.38} \leq 1.0
\end{aligned}
$$

For pile 3, equation (5-3) is valid with

$$
\begin{aligned}
& \beta_{1}=0.48\left(\frac{r_{23}}{2 r_{p}}\right)^{0.38} \leq 1.0 \\
& \beta_{2}=0.48\left(\frac{r_{12}+r_{23}}{2 r_{p}}\right)^{0.38} \leq 1.0
\end{aligned}
$$

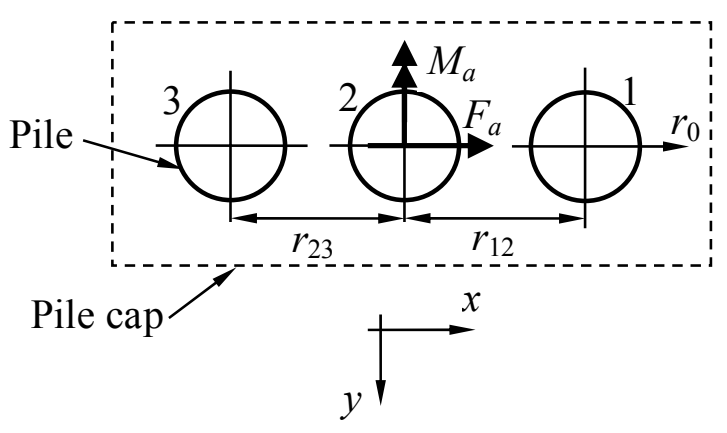

Figure 5-2 Three-Pile Group 
Figure 5-3 describes the geometry of the four-pile group. The coefficient for piles 1 and 2 is the same, while the coefficient for piles 3 and 4 is the same. The coefficient $f$ for pile 1 or 2 can be expressed as:

$$
f=\beta_{1} \beta_{2} \beta_{3}
$$

with

$$
\begin{aligned}
& \beta_{1}=0.7\left(\frac{r_{23}}{2 r_{p}}\right)^{0.26} \leq 1.0 \\
& \beta_{2}=0.64\left(\frac{r_{12}}{2 r_{p}}\right)^{0.34} \leq 1.0 \\
& \beta_{3}=\sqrt{\beta_{1}^{2} \cos ^{2} \alpha+\beta_{2}^{2} \sin ^{2} \alpha} \leq 1.0
\end{aligned}
$$

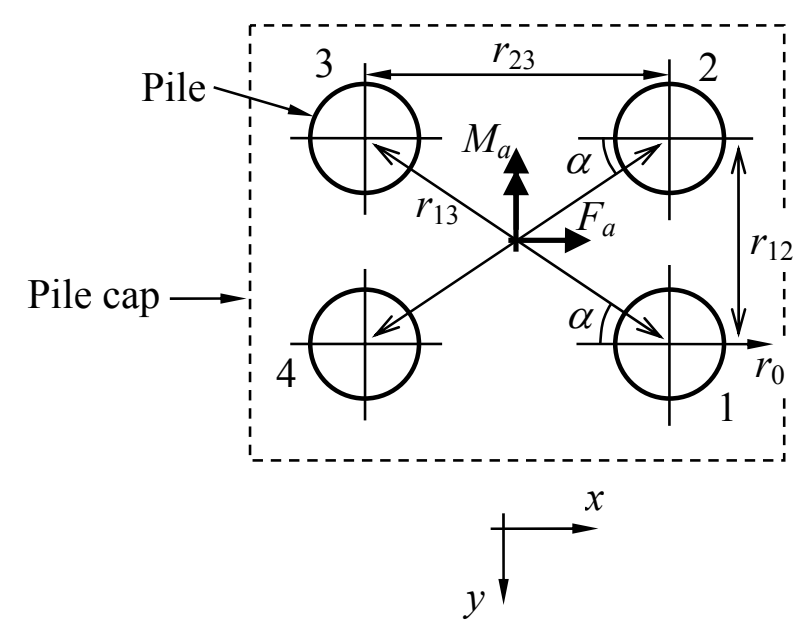

Figure 5-3 Four-Pile Group

For pile 3 or 4 , equation (5-9) is valid with

$$
\begin{aligned}
& \beta_{1}=0.48\left(\frac{r_{23}}{2 r_{p}}\right)^{0.38} \leq 1.0 \\
& \beta_{2}=0.64\left(\frac{r_{12}}{2 r_{p}}\right)^{0.34} \leq 1.0
\end{aligned}
$$


$\beta_{3}=\sqrt{\beta_{1}^{2} \cos ^{2} \alpha+\beta_{2}^{2} \sin ^{2} \alpha} \leq 1.0$

The six-pile group is described by Figure 5-4. The factor $f$ for pile 1 or 2 is given by $f=\beta_{1} \beta_{2} \beta_{3} \beta_{4} \beta_{5}$

with

$$
\begin{aligned}
& \beta_{1}=0.7\left(\frac{r_{23}}{2 r_{p}}\right)^{0.26} \leq 1.0 \\
& \beta_{2}=0.7\left(\frac{r_{23}+r_{46}}{2 r_{p}}\right)^{0.26} \leq 1.0 \\
& \beta_{3}=0.64\left(\frac{r_{12}}{2 r_{p}}\right)^{0.34} \leq 1.0 \\
& \beta_{4}=\sqrt{\beta_{2}^{2} \cos ^{2} \delta+\beta_{3}^{2} \sin ^{2} \delta} \leq 1.0 \\
& \beta_{5}=\sqrt{\beta_{1}^{2} \cos ^{2} \alpha_{1}+\beta_{3}^{2} \sin ^{2} \alpha_{1}} \leq 1.0
\end{aligned}
$$

For pile 3 or 4 , equation (5-16) is valid with

$$
\begin{aligned}
& \beta_{1}=0.48\left(\frac{r_{23}}{2 r_{p}}\right)^{0.38} \leq 1.0 \\
& \beta_{2}=0.7\left(\frac{r_{46}}{2 r_{p}}\right)^{0.26} \leq 1.0 \\
& \beta_{3}=0.64\left(\frac{r_{12}}{2 r_{p}}\right)^{0.34} \leq 1.0 \\
& \beta_{4}=\sqrt{\beta_{2}^{2} \cos ^{2} \alpha_{2}+\beta_{3}^{2} \sin ^{2} \alpha_{2}} \leq 1.0 \\
& \beta_{5}=\sqrt{\beta_{1}^{2} \cos ^{2} \alpha_{1}+\beta_{3}^{2} \sin ^{2} \alpha_{1}} \leq 1.0
\end{aligned}
$$

For pile 5 or 6 , equation (5-16) is again valid with

$$
\beta_{1}=0.48\left(\frac{r_{23}+r_{46}}{2 r_{p}}\right)^{0.38} \leq 1.0
$$




$$
\begin{aligned}
& \beta_{2}=0.48\left(\frac{r_{46}}{2 r_{p}}\right)^{0.38} \leq 1.0 \\
& \beta_{3}=0.64\left(\frac{r_{12}}{2 r_{p}}\right)^{0.34} \leq 1.0 \\
& \beta_{4}=\sqrt{\beta_{2}^{2} \cos ^{2} \alpha_{2}+\beta_{3}^{2} \sin ^{2} \alpha_{2}} \leq 1.0 \\
& \beta_{5}=\sqrt{\beta_{1}^{2} \cos ^{2} \delta+\beta_{3}^{2} \sin ^{2} \delta} \leq 1.0
\end{aligned}
$$

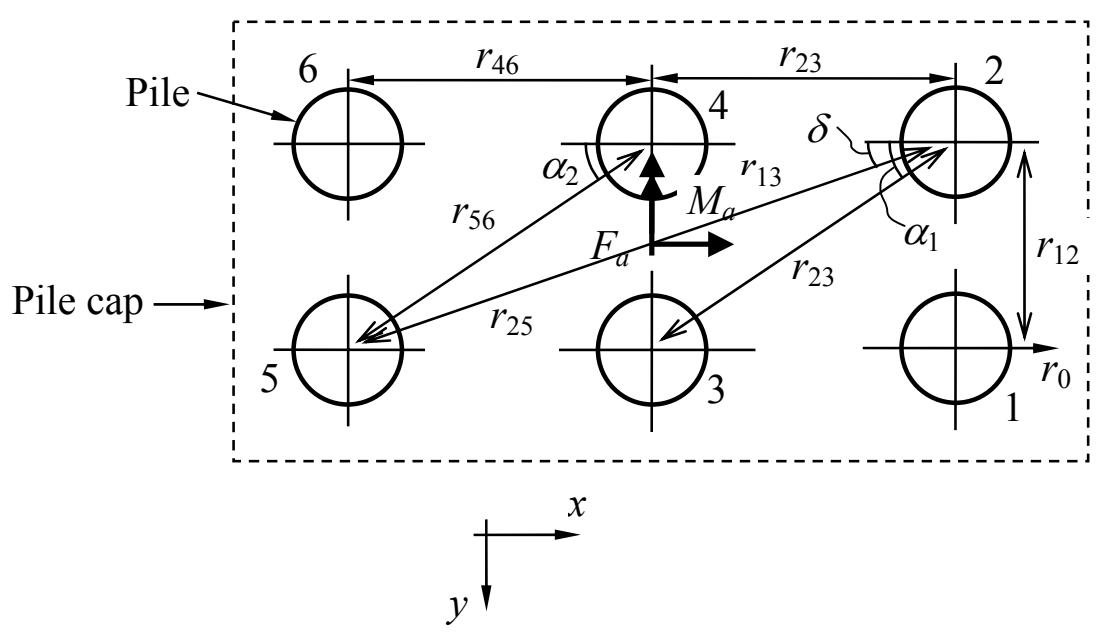

Figure 5-4 Four-Pile Group

\subsection{Results}

In order to illustrate the pile group analysis, we consider the soil profile of the example problem of Figure 4-9 (clay layers overlain by a sand layer); however, instead of a single pile, we assume a four-pile group with a center-to-center spacing of $2.5 \mathrm{~m}$. All the piles in the group are assumed to be $15 \mathrm{~m}$ long with diameters equal to $1.0 \mathrm{~m}$. We restrain the rotation at the head of all the piles with the assumption that the piles are attached to a rigid pile cap. Figure 5-5 shows the head deflections of the leading and trailing piles as a function of applied force. Also plotted in the figure is the head deflection of a single pile (i.e., without a group) with a restrained head condition that has the same length and diameter as those of the piles in the group. It is evident that the leading pile has a slightly stiffer response than the trailing pile. In general, the 
pile group deflects more than a single pile. For example, if we want to restrict the head deflection at $10 \mathrm{~mm}$, say, then the corresponding load at the head of the leading pile is $155 \mathrm{kN}$, while that at the head of the trailing pile is $125 \mathrm{kN}$. Thus, a maximum total load of $2 \times(155+$ $125)=560 \mathrm{kN}$ can be applied on the group. In contrast, the maximum load that can be applied for a $10 \mathrm{~mm}$ deflection for a single pile is $165 \mathrm{kN}$.

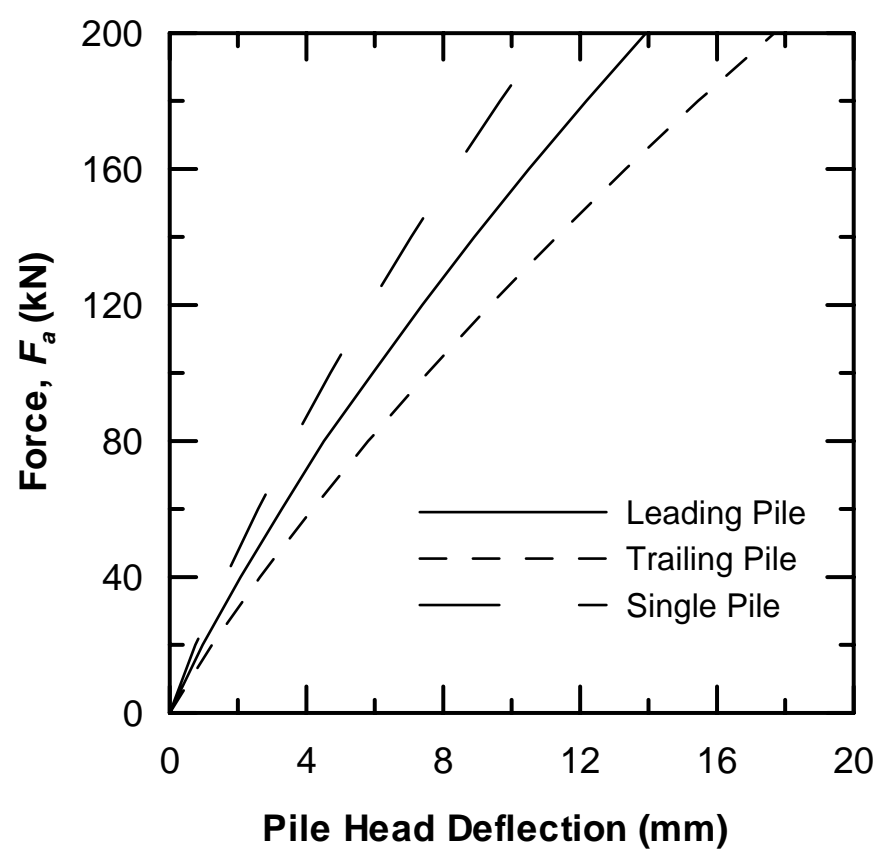

Figure 5-5 Six-Pile Group

\subsection{Summary}

In this chapter, we propose a method for analysis of pile groups. The method is an extension of the single pile analysis. Different coefficients (depending on the position of a pile in a group and the number of piles in a group) are proposed that modify the soil resistances obtained from single pile analysis for different pile groups. An example is provided to illustrate the method. 


\section{CHAPTER 6. RETROSPECTION AND RECOMMENDATIONS}

\subsection{Introduction}

In this final chapter, we present a summary of the research presented in the previous chapters. We also present the main conclusions. Finally, we give recommendations for future extensions of this research.

\subsection{Summary}

In this research study, we developed a continuum-based analysis of piles embedded in multiple soil layers and subjected to a horizontal force and moment at the pile head. The pile is modeled as an Euler-Bernoulli beam while the soil surrounding the pile is modeled as either a linear elastic or a nonlinear elastic material. The displacements in the soil are described as products of separable variables with different dimensionless shape functions for radial and tangential displacements accounting for the decay in the soil displacements with increasing radial distance from the pile. The differential equations governing the equilibrium configuration of the pile-soil system were derived applying the principle of minimum potential energy. The differential equation of pile deflection was solved analytically while the differential equations of soil displacements were solved using the one-dimensional finite difference method. An iterative solution algorithm was developed that ensured the global equilibrium of the pile-soil system. The method takes into account the three-dimensional interaction of the pile with the surrounding soil and produces pile response comparable with that from three-dimensional (3D) finite element method (FEM); however, the computational effort required by the method is much less than that required by an equivalent 3D FEM (nonlinear pile response is produced within minutes). Analysis of pile group was also performed by modifying the soil resistance depending on the number of piles in a group and the relative positions of the piles

In chapter 1, we presented a brief overview of piles foundations. We discussed the statics and kinematics of pile response against vertical and lateral loads and discussed the soil spring 
approach and the continuum approach of analysis of laterally loaded piles. We established that the continuum approach is conceptually more appropriate for analysis of laterally loaded piles; this set the stage for the analysis that was performed as a part of this research. In chapter 2, we showed that a simple beam-on-elastic foundation approach can also be used to analyze laterally loaded piles as a prologue to the continuum analysis subsequently performed. It revealed the analogy between the soil-spring approach and the continuum approach. An analytical method of solving differential equations, the method of initial parameters, was modified and applied to the governing differential equation of pile deflection. In chapter 3, the continuum-based elastic analysis for a single, circular pile was presented. The differential equations for pile deflection and soil displacements were systematically developed using the principle of minimum potential energy and calculus of variations. An iterative solution algorithm was used. The linear elastic analysis was extended to take into account the soil nonlinearity in chapter 4 . Different constitutive models describing soil nonlinearity were first introduced and then the mathematical analysis developed for linear soil was modified to produce the nonlinear pile response. Pile groups were analyzed in chapter 5 by incorporating into the analysis modified soil resistance as a function of the number of piles in a group and of the relative position of the piles.

Through this research, we have demonstrated that a complex three-dimensional boundary value problem, that have so far been analyzed either by simple soil-spring approach or expensive three-dimensional numerical analysis, can be solved with minimal computational effort yet maintaining sufficient rigor so that the essential features of the pile-soil interaction are captured. The need for representing soil resistance by springs with empirical spring constants (which, in the case of nonlinearity, depend on pile deflection) is completely eliminated as the resistance is rigorously calculated using integral equations that are functions of soil modulus and of the rate of decrease of soil displacements with radial distance from the pile. The unique feature of this analysis is that, along with the pile response (i.e., pile deflection, bending moment and shear force), displacements and strains in the surrounding soil can also be obtained. Such a detailed analysis has so far been possible only by the use of three-dimensional numerical analysis like the finite element analysis, which is expensive and cannot be performed routinely for design. By the use of this analysis, pile response is obtained semi-analytically in minutes and can be readily used in design calculations. The inputs required for the analysis are pile geometry and modulus; and the soil properties, typically the soil Poisson's ratio, the void ratio, and the number and 
thickness of soil layers, that can be determined from simple in situ or laboratory tests. The inputs can be typed in a text file requiring minimum effort on the part of the user.

\subsection{Future Research}

Any new analysis method requires sufficient checks before it can be accepted in practice. In the above research, the analysis has been compared with the exiting p-y method. However, the p-y method cannot truly represent soil behavior, and moreover, the present analysis produces soil displacements and strains along side pile deflection, which the p-y method cannot produce. Thus, we need a systematic way of validating the analysis developed in the research, which will check the pile deflections, and soil displacements and strains. An important point to note is that, unlike the p-y method, the analysis uses soil parameters that can be estimated from routine tests. Controlled pile load tests, in which soil properties are estimated using routine tests, is an appropriate way to validate the analysis. That will help us to estimate the soil resistance with reasonable accuracy, which is often not possible in real field problems due to inadequate site characterization. Thus, we recommend the development of a load testing program. The Bowen laboratory at Purdue University has the facility for performing laboratory-scale pile load tests under controlled conditions. It is envisaged that the facility can be used successfully to develop the testing program. 


\section{LIST OF REFERENCES}

Aköz, Y., Öntuna, K. A. \& Sağlamer, A. (1981). A new approach to the analysis of laterally loaded piles. Proc. $10^{\text {th }}$ Int. Conf. Soil Mech. Fdn. Engng. 2, Stolkholm, 587-592.

American Petroleum Institute. (1993). Recommended practice for planning, designing and constructing fixed offshore platforms - working stress design, API-RP-2A, $20^{\text {th }}$ edition.

Anagnostopoulos, C. \& Georgiadis, M. (1993). Interaction of axial and lateral pile responses. Geotech. Engng., Am. Soc. Civ. Engrs. 119, No. 4, 793-798.

Anderson, J. B. \& Townsend, F. C. (2001). SPT and CPT testing for evaluating lateral loading of deep foundations. J. Geotech. Geoenv. Engng., Am. Soc. Civ. Engrs. 127, No. 11, 920-925.

Anderson, J. B., Townsend, F. C. \& Grajales, B. (2003). Case history evaluation of laterally loaded piles. J. Geotech. Geoenv. Engng., Am. Soc. Civ. Engrs. 129, No. 3, 187-196.

Ashford, S. C. \& Juirnarongrit, T. (2003). Evaluation of pile diameter effect on initial modulus of subgrade reaction. J. Geotech. Geoenv. Engng., Am. Soc. Civ. Engrs. 129, No. 3, 234-242.

Ashour, M. \& Norris, G. (2000). Modeling lateral soil-pile response based on soil-pile interaction. J. Geotech. Geoenv. Engng., Am. Soc. Civ. Engrs. 126, No. 5, 420-428.

Ashour, M., Norris, G. \& Pilling, P. (1998). Lateral loading of a pile in layered soil using the strain wedge model. J. Geotech. Geoenv. Engng., Am. Soc. Civ. Engrs. 124, No. 4, 303-315.

Ashour, M., Pilling, P. \& Norris, G. (2001). Assessment of pile group response under lateral load. Proc. 4th Int. Conf. Recent Advan. Geotech. Engng., San Diego, 1-6.

Ashour, M., Pilling, P. \& Norris, G. (2004). Lateral behavior of pile groups in layered soils. $J$. Geotech. Geoenv. Engng., Am. Soc. Civ. Engrs. 130, No. 6, 580-592.

Baguelin, F., Frank, R. \& Saĭd, Y. H. (1977). Theoretical study of lateral reaction mechanism of piles. Geotechnique 27, No. 3, 405-434.

Banerjee, P. K. (1978). Analysis of axially and laterally loaded pile groups. Develop. Soil Mech., 1, Ed. Scott, C. R., Applied Science Pub. Ltd. Lond., 317-346.

Banerjee, P. K. \& Davies, T. G. (1978). The behavior of axially and laterally loaded single piles embedded in nonhomogeneous soils. Geotechnique 28, No. 3, 309-326. 
Banerjee, P. K. \& Davies, T. G. (1980). Analysis of some reported case histories of laterally loaded pile groups. Num. Meth. Offshore Piling, Inst. Civ. Engrs. 101-108.

Basile, F. (1999). Non-linear analysis of pile groups. Proc. Instn. Civ. Engrs. Geotech. Engng. $137,105-115$.

Basu, D. (2006). Analysis of laterally loaded piles in layered soil. Ph.D. Thesis, Purdue University.

Basu, D. \& Salgado, R. (2007a). Elastic analysis of laterally loaded pile in multi-layered soil. Geomech. Geongng. Int. J. 2, No. 3, 183-196.

Basu, D. \& Salgado, R. (2007b). Method of initial parameters for laterally loaded piles embedded in layered Soils. Geomech. Geongng. Int. J. 2, No. 4, 281-294.

Bhowmik, S. K. \& Long, J. H. (1991). An analytical investigation of the behavior of laterally loaded piles. Proc. Congress Geotech. Engng. Div. 2, Am. Soc. Civ. Engrs, 1307-1318.

Biot, M. A. (1937). Bending of an infinite beam on an elastic foundation. J. Appl. Mech., Trans. Am. Soc. Mech. Engrs 4, No. 1, A1-A7.

Block. H. D. (1962). Introduction to tensor analysis. Charles E. Merrill Books, Inc.

Bogard, D \& Matlock, H. (1983). Procedures for analysis of laterally loaded pile groups in soft clay. Proc. Geotech. Practice In Offshore Engng., Am. Soc. Civ. Engrs., Texas, 499-535.

Bolton, M. D. (1986). Strength and dilatancy of sands. Geotechnique 36, No. 1, 65-78.

Bowles, J. (1997). Foundation analysis and design. McGraw-Hill.

Bowman, F. (1958). Introduction to Bessel functions. Dover Publications, Inc.

Bransby, M. F. (1996). Difference between load-transfer relationships for laterally loaded pile groups: active $p-y$ or passive $p-\delta$. J. Geotech. Engng., Am. Soc. Civ. Engrs. 122, No. 12, 10151018.

Bransby, M. F. (1999). Selection of p-y curves for the design of single laterally loaded piles. Int. J. Num. Anal. Meth. Geomech. 23, 1909-1926.

Briaud, J.-L. (1997). Sallop: simple approach for lateral loads on piles. J. Geotech. Geoenv. Engng., Am. Soc. Civ. Engrs. 123, No. 10, 958-964.

Broms, B. B. (1964a). Lateral resistance of piles in cohesive soils. J. Soil Mech. Fdn. Div., Am. Soc. Civ. Engrs. 90, No. SM2, 27-63. 
Broms, B. B. (1964b). Lateral resistance of piles in cohesionless soils. J. Soil Mech. Fdn. Div., Am. Soc. Civ. Engrs. 90, No. SM3, 123-156.

Broms, B. B. (1965). Design of laterally loaded piles. J. Soil Mech. Fdn. Div., Am. Soc. Civ. Engrs. 91, No. SM3, 79-99.

Brown, D. A. \& Kumar, M. (1989). P-y curves for laterally loaded piles derived from threedimensional finite element model. Numer. Models Geomech. NUMOG III, Ed. Pietruszak, S. \& Pande, G.N., Elsevier Applied Science, 683- 690.

Brown, D. A. \& Shie, C. F. (1991). Modification of p-y curves to account for group effects on laterally loaded piles. Proc. Congress Geotech. Engng. Div. 1, Am. Soc. Civ. Engrs., 479-489.

Brown, D. A., Reese, L. C. \& O'Neill, M. W. (1987). Cyclic lateral loading of a large-scale pile group. J. Geotech. Engng., Am. Soc. Civ. Engrs. 113, No. 11, 1326-1343.

Brown, D. A., Morrison, C. \& Reese, L. C. (1988). Lateral load behavior of pile group in sand. $J$. Geotech. Engng., Am. Soc. Civ. Engrs. 114, No. 11, 1261-1276.

Brown, D. A., Hidden, S. A. \& Zhang, S. (1994). Determination of $P-Y$ curves using inclinometer data. Geotech. Test. J., GTJODJ, 17, No. 2, 150-158.

Budhu, M. \& Davies, T. G. (1988). Analysis of laterally loaded piles in soft clays. J. Geotech. Engng. Div., Am. Soc. Civ. Engrs. 114, No. 1, 21-39.

Budiman, J. \& Ahn, K. (2005). Effects of pile cap in single pile and lateral capacity of pile groups. Geo-Frontiers 2005, GSP 132, CD ROM.

Chandrasekharaiah, D. S. \& Debnath, L. (1994). Continuum Mechanics. Academic Press.

Chapra, S. C. \& Canale, R. P. (1998). Numerical methods for engineers. WCB/McGraw-Hill.

Chow, Y. K. (1987). Axial and lateral response of pile groups embedded in nonhomogeneous soils. Int. J. Numer. Anal. Meth. Geomech. 11, 621-638.

Comodromos, E. M. \& Pitilakis, K. D. (2005). Response evaluation for horizontally loaded fixed-head pile groups using 3-D non-linear analysis. Int. J. Numer. Anal. Meth. Geomech. 29, 597-625.

Cox, W. R., Dixon, D. A. \& Murphy, B. S. (1984). Lateral-load tests on 25.4-mm (1-in.) diameter piles in very soft clay in side-by-side and in-line groups. Laterally Loaded Deep Fdns., ASTM STP 835, 122-139.

Davies, T. G. \& Budhu, M. (1986). Non-linear analysis of laterally loaded piles in heavily overconsolidated clays. Geotechnique 36, No. 4, 527-538. 
Davis, R. O. \& Selvadurai, A. P. S. (1996). Elasticity and geomechanics. Cambridge Univ. Press.

Davis, R. O. \& Selvadurai, A. P. S. (2002). Plasticity and geomechanics. Cambridge Univ. Press.

Davisson, M. T. (1970). Lateral load capacity of piles. Highway Res. Rec. 333, 104-112.

Davisson, M. T. \& Gill, H. L. (1963). Laterally loaded piles in a layered soil system. J. Soil Mech. Fdn. Div., Am. Soc. Civ. Engrs. 89, No. SM3, 63-94.

Desai, C. S. \& Appel, G. C. (1976). 3-D analysis of laterally loaded structures. Num. Meth. Geomech., Am. Soc. Civ. Engrs. Ed. Desai, C.S., 2, 405-418.

Duncan, M., Evans, L. T. \& Ooi, P. S. K. (1994). Lateral load analysis of single piles and drilled shafts. J. Geotech. Engng., Am. Soc. Civ. Engrs. 120, No. 5, 1018-1033.

Dunnuvant, T. W. \& O’Neill, M. W. (1986). Evaluation of design-oriented methods for analysis of vertical pile groups subjected to lateral load. Proc. $3^{\text {rd }}$ Intl. Conf. Num. Meth. Offshore Piling, Paris, 303-316.

Einav, I. (2005). Energy and variational principles for piles in dissipative soil. Geotechnique 55, No. 7, 515-525.

Fahey, M. \& Carter, J. P. (1993). A finite element study of the pressuremeter test in sand using a nonlinear elastic plastic model. Can. Geotech. J. 30, 348-362.

Feagin, L. B. (1935). Lateral pile-loading tests. Trans. Am. Soc. Civ. Engrs., 236-254.

Fleming, W. G. K., Weltman, A. J., Randolph, M. F. \& Elson, W. K. (1992). Blackie \& Son Ltd.

Focht, J. A. \& Koch, K. J. (1973). Rational analysis of the lateral performance of offshore pile groups. Proc. 5th Offshore Tech. Conf. 2, Houston, Texas, 701-708.

Francis, A. (1964). Analysis of pile groups with flexural resistance. J. Soil Mech. Fdn. Div., Am. Soc. Civ. Engrs. 90, No. SM3, 1-32.

Fung, Y. C. (1994). A first course in continuum mechanics for physical and biological engineers and scientists. Prentice-Hall, Inc.

Fung, Y. C. \& Tong, P. (2001). Classical and computational solid mechanics. World Scientific Publishing Co. Pte. Ltd., Singapore.

Gabr, M. A., Lunne, T. \& Powell, J. J. (1994). P-y analysis of laterally loaded piles in clay using DMT. J. Geotech. Engng., Am. Soc. Civ. Engrs. 120, No. 5, 816-837.

Gandhi, S. R. \& Selvam, S. (1997). Group effect on driven piles under lateral load. J. Geotech. Geoenv. Engng., Am. Soc. Civ. Engrs. 123, No. 8, 702-709. 
Georgiadis, M. (1983). Development of p-y curves for layered soils. Proc. Conf. Geotech. Pract. Offshore Engng., Am. Soc. Civ. Engrs. 536-545.

Georgiadis, M. \& Butterfield, R. (1982). Laterally loaded pile behavior. J. Geotech. Engng. Div., Am. Soc. Civ. Engrs. 108, No. GT1, 155-165.

Georgiadis, M., Anagnostopoulos, C. \& Saflekou, S. (1992). Centrifugal testing of laterally loaded piles in sand. Can. Geotech. J. 29, 208-216.

Guo, W. D. \& Lee, F. H. (2001). Load transfer approach for laterally loaded piles. Int. J. Numer. Anal. Meth. Geomech. 25, No. 11, 1101-1129.

Hardin, B. O. (1978). The nature of stress-strain behavior for soils. Earthquake Engng. Soil Dyn., Am. Soc. Civ. Engrs. 1, 3-90.

Hardin, B. O. \& Black, W. L. (1966). Sand stiffness under various triaxial stresses. J. Soil Mech. Fdn. Div., Am. Soc. Civ. Engrs. 92, No. SM2, 27-42.

Hardin, B. O. \& Black, W. L. (1968). Vibration modulus of normally consolidated clay. J. Soil Mech. Fdn. Div., Am. Soc. Civ. Engrs. 94, No. SM2, 353-369.

Hardin, B. O. \& Drnevich, V. P. (1972). Shear modulus and damping in soils: design equations and curves. J. Soil Mech. Fdn. Div., Am. Soc. Civ. Engrs. 98, No. SM7, 667-692.

Hariharan, M. \& Kumarasamy, K. (1983). Analysis of pile groups subjected to lateral loads. Proc. $3^{\text {rd }}$ Int. Conf. Behav. Meth. Offshore Struct., Massachusetts, 383-390.

Harr, M. E., Davidson, J. L., Ho, D.-M., Pombo, L. E., Ramaswamy, S. V. \& Rosner, J. C. (1969). Euler beams on a two parameter foundation model. J. Soil Mech. Fdn. Div., Am. Soc. Civ. Engrs 95, No. SM4, 933-948.

Hetényi, M. (1946). Beams on elastic foundation. Univ. Michigan Press, Ann Arbor.

Holloway, D. M., Moriwaki, Y., Stevens, J. B. \& Perez, J. Y. (1981). Response of a pile group to combined axial and lateral loading. Proc. 10 ${ }^{\text {th }}$ Int. Conf. Soil Mech. Fdn. Engng., 2, Stockholm, 731-734.

Horsnell, M. R., Aldridge, T. R. \& Erbrich, C. E. (1990). Lateral group behavior of piles in offshore soil conditions. Proc. $22^{\text {nd }}$ Offshore Tech. Conf., Houston, Texas, 417-424.

Hsiao, J. K. \& Kumar, S. (2005). Predicted and measured response of precast concrete piles under lateral load. Electronic J. Geot. Engrg.

<_http://www.ejge.com/2005/ppr0607/ppr0607.htm> (November 13, 2006).

Hsiung, Y. (2003). Theoretical elastic-plastic solution for laterally loaded piles. J. Geotech. Geoenv. Engng., Am. Soc. Civ. Engrs. 129, No. 6, 475-480. 
Hsiung, Y. \& Chen, Y. (1997). Simplified method for analyzing laterally loaded single piles in clays. J. Geotech. Geoenv. Engng., Am. Soc. Civ. Engrs. 123, No. 11, 1018-1029.

Hsiung, Y.-M., Chen, S.-S. \& Chou, Y.-C. (2006). Analytical solution for piles supporting combined lateral loads. J. Geotech. Geoenv. Engng., Am. Soc. Civ. Engrs. 132, No. 10, 13151324.

Huang, A-B, Hsueh, C-K, O’Neill, M. W., Chern, S. \& Chen, C. (2001). Effects of construction on laterally loaded pile groups. J. Geotech. Geoenv. Engng., Am. Soc. Civ. Engrs. 127, No. 5, 385-397.

Huangfu, F.-M., Wang, Y.-Q. \& Zhang, J. (2003). Behavior of pile under combined axial and lateral loading. J. Harbin Inst. Tech. 35, No. 6. 743-746.

Illyas, T., Leung, C. F., Chow, Y. K. \& Budi, S. S. (2004). Centrifuge model study of laterally loaded pile groups in clay. J. Geotech. Geoenv. Engng., Am. Soc. Civ. Engrs. 130, No. 3, 274283.

Ishibashi, I. \& Zhang, X. (1993). Unified dynamic shear moduli and damping ratios of sand and clay. Soils Fdns. 33, No. 1, 182-191.

Ismael, N. F. \& Klym, T. W. (1978). Behavior of rigid piers in layered cohesive soils. J. Geotech. Engng. Div., Am. Soc. Civ. Engrs 104, No. GT8, 1061-1074.

Jones, R. \& Xenophontos, J. (1977). The Vlasov foundation model. Int. J. Mech. Sci 19, 317-323.

Kerr, A. D. (1964). Elastic and viscoelastic foundation models. J. Appl. Mech., Trans. Am. Soc. Mech. Engrs 31, No. 3, 491-498.

Kim, J. B. \& Brungraber, R. J. (1976). Full-scale lateral load tests of pile groups. J. Geotech. Engng. Div., Am. Soc. Civ. Engrs. 102, No. GT1, 87-105.

Kim, T. K., Kim, N-K, Lee, W. J. \& Kim, Y. S. (2004). Experimental load-transfer curves of laterally loaded piles in Nak-Dong river sand. J. Geotech. Geoenv. Engng., Am. Soc. Civ. Engrs. 130, No. 4, 416-425.

Kondner, R. L. (1963). Hyperbolic stress-strain response: cohesive soils. J. Soil Mech. Fdn. Div., Am. Soc. Civ. Engrs. 89, No. SM1, 115-143.

Kooijman, A. P. (1989). Comparison of an elastoplastic quasi three-dimensional model for laterally loaded piles with field tests. Numer. Models Geomech. NUMOG III, Ed. Pietruszak, S. \& Pande, G.N., Elsevier Applied Science, 675- 682.

Kooijman, A. P. \& Vermeer, P. A. (1988). Elastoplastic analysis of laterally loaded piles. Proc. $6^{\text {th }}$ Int. Conf. Num. Meth. Geomech., Innsbruck, Austria. 2, 1033-1042. 
Langhaar, H. L. (1989). Energy methods in applied mechanics. Robert E. Krieger Publishing Co., Inc.

Law, H. K. \& Lam, I. P. (2001). Application of periodic boundary for large pile group. $J$. Geotech. Geoenv. Engng., Am. Soc. Civ. Engrs. 127, No. 10, 889-892.

Lee, J. \& Salgado, R. (2000). Analysis of calibration chamber plate load tests. Can. Geotech. J. $37,14-25$.

Lee, C. Y. \& Small, J. C. (1991). Finite layer analysis of laterally loaded piles in crossanisotropic soils. Int. J. Numer. Anal. Meth. Geomech. 15, 785-808.

Lee, S. L., Kog, Y. C. \& Karunaratne, G. P. (1987). Laterally loaded piles in layered soil. Soils Fdns. 27, No. 4, 1-10.

Leung, C. F. \& Chow, Y. K. (1987). Response of pile groups subjected to lateral loads. Int. J. Numer. Anal. Meth. Geomech. 11, 307-314.

Loukidis, D. (2006).Advanced constitutive modeling of sands and applications to foundation engineering. Ph.D. Thesis, Purdue University.

Lubliner, J. (1990). Plasticity theory. Macmillan Publishing Company.

Madhav, M. R., Rao, N. S. V. K. \& Madhavan, K. (1971). Laterally loaded pile in elasto-plastic soil. Soils Fdns. 11, No. 2, 1-15.

Maharaj, D. K. (2003) Load-Deflection Response of Laterally Loaded Single Pile by Nonlinear Finite Element Analysis. Electronic J. Geot. Engrg.

< http://www.ejge.com/2003/ppr0342/ppr0342.htm> (November 13, 2006).

Mal, A. K. \& Singh, S. J. (1991). Deformation of elastic solids. Prentice-Hall, Inc.

Malvern, L. E. (1969). Introduction to the mechanics of a continuous medium. Prentice-Hall, Inc.

Matlock, H. (1970). Correlations for design of laterally loaded piles in soft clay. Proc. $2^{\text {nd }}$ Offshore Tech. Conf., Houston, Texas, 1, 577-594.

Matlock, H. \& Reese, L. C. (1960). Generalized solutions for laterally loaded piles. J. Soil Mech. Fdn. Div., Am. Soc. Civ. Engrs. 86, No. SM5, 63-91.

Mayne, P. W. (2000). Enhanced Geotechnical Site Characterizatin by Seismic Piezocone Penetration Tests. Proc. $4^{\text {th }}$ Int. Geot. Conf., Cairo University, 95-120.

$<$ http://www.geotechlinks.com/download.php?id=GL0138> (December 1, 2006).

McClelland, B. \& Focht Jr., J. A. (1958). Soil modulus for laterally loaded piles. Trans. Am. Soc. Civ. Engrs. 123, 1049-1063. 
McLachlan, N. W. (1961). Bessel functions for engineers. Oxford Univ. Press.

McVay, M., Casper, R. \& Shang, T. I. (1995). Lateral response of three-row groups in loose to dense sands at 3D and 5D pile spacing. J. Geotech. Engng., Am. Soc. Civ. Engrs. 121, No. 5, 436-441.

McVay, M., Bloomquist, D., Vanderlinde, D. \& Clausen, J. (1994). Centrifuge modeling of laterally loaded pile groups in sands. Geotech. Test. J. GTJODJ, 17, No. 2, 129-137.

McVay, M. C., Shang, T. I. \& Casper, R. (1996). Centrifuge testing of fixed-head laterally loaded battered and plumb pile groups in sand. Geotech. Test. J. GTJODJ, 19, No. 1, 41-50.

McVay, M., Zhang, L., Molnit, T. \& Lai, P. (1998). Centrifuge testing of large laterally loaded pile groups in sands. J. Geotech. Geoenv. Engng., Am. Soc. Civ. Engrs. 124, No. 10, 1016-1026.

Mindlin, R. D. (1936). Force at a point in the interior of a semi-infinite solid. Physics 7, May, 195-202.

Mokwa, R. L. (1999). "Investigation of the resistance of pile caps to lateral loading." Ph.D. Thesis, Virginia Polytechnic Institute and State University.

Mokwa, R. L. \& Duncan, J. M. (2001). Experimental evaluation of lateral-load resistance of pile caps. J. Geotech. Geoenv. Engng., Am. Soc. Civ. Engrs. 127, No. 2, 185-192.

Naylor, D. J., Pande, G. N., Simpson, B. \& Tabb, R. (1981). Finite elements in geotechnical engineering. Pineridge Press Ltd., Swansea, U. K.

Ng, C. W. W., Rigby, D. B. Ng, S. W. L. \& Lei, G. H. (2000). Field studies of well-instrumented barrette in Hong Kong. J. Geotech. Geoenv. Engng., Am. Soc. Civ. Engrs. 126, No. 1, 60-73.

Ng C. W. W., Zhang, L. \& Nip, D. C. N. (2001). Response of laboratory loaded large-diameter bored pile groups. J. Geotech. Geoenv. Engng., Am. Soc. Civ. Engrs. 127, No. 8, 658-669.

Nogami, T. \& Paulson, S. K. (1985). Transfer matrix approach for nonlinear pile group response analysis. Int. J. Numer. Anal. Meth. Geomech. 9, 299-316.

O’Neill, M. W. \& Murchinson, J. M. (1983). An evaluation of $p-y$ relationships in sand. Rep. prepared for American Petroleum Inst., Washington, D.C. (as cited in Kim et al. 2004).

O’Neill, M. W., Ghazzaly, O. I. \& Ha, H. B. (1977). Analysis of three-dimensional pile groups with non-linear soil response and pile-soil-pile interaction. Proc. $9^{\text {th }}$ Offshore Tech. Conf., Houston, Texas, 2, 245-256.

O’Neill, M. W., Reese, L. C. \& Cox, W. R. (1990). Soil behavior for piles under lateral loading. Proc. $22^{\text {nd }}$ Offshore Tech. Conf., Houston, Texas, 3, 279-287. 
Ooi, P. S. K. \& Duncan, J. M. (1994). Lateral load analysis of groups of piles and drilled shafts. J. Geotech. Engng., Am. Soc. Civ. Engrs. 120, No. 6, 1034-1050.

Ooi, P. S. K., Chang, B. K. F. \& Wang, S. (2004). Simplified lateral load analyses of fixed-head piles and pile groups. J. Geotech. Geoenv. Engng., Am. Soc. Civ. Engrs. 130, No. 11, 1140-1151.

Pile Buck. (2003). The pile driving, foundation and marine contractor's newspaper. April.

$<$ http://www.pilebuck.com/newspaper/> (November 13, 2006).

Pise, P. J. (1982). Laterally loaded piles in a two-layer soil system. J. Geotech. Engng. Div., Am. Soc. Civ. Engrs. 108, No. GT9, 1177-1181.

Poulos, H. G. (1971a). Behavior of laterally loaded piles: I - single piles. J. Soil Mech. Fdn Div., Am. Soc. Civ. Engrs. 97, No. SM5, 711-731.

Poulos, H. G. (1971b). Behavior of laterally loaded piles: II - pile groups. J. Soil Mech. Fdn Div., Am. Soc. Civ. Engrs. 97, No. SM5, 733-751.

Poulos, H. G. (1972). Behavior of laterally loaded piles: III - socketed piles. J. Soil Mech. Fdn Div., Am. Soc. Civ. Engrs. 98, No. SM4, 341-360.

Poulos, H. G. (1973). Load-deflection prediction for laterally loaded piles. Aust. Geomech. J. G3, No. 1, 1-8.

Poulos, H. G. (1975) Lateral load-deflection prediction for pile groups. J. Geotech. Engng. Div., Am. Soc. Civ. Engrs. 101, No. GT1, 19-34.

Poulos, H. G. \& Davis, E. H. (1980). Pile foundation analysis and design. John Wiley \& Sons, Inc.

Prakash, S. \& Sharma, H. D. (1990). Pile foundations in engineering practice. John Wiley \& Sons, Inc.

Pyke, R. \& Beikae, M. (1984). A new solution for the resistance of single piles to lateral loading. Laterally Loaded Deep Fdns., ASTM STP 835, 3-20.

Qiu, T., Fox, P. J., Janoyan, K., Stewart, J. P. \& Wallace, J. W. (2004). Geo-Support 2004, Drilled Shafts, Micropiling, Deep Mixing, Remedial Methods, and Specialty Foundation Systems, GSP 124, 613-624.

Randolph, M. F. (1981). The response of flexible piles to lateral loading. Geotechnique 31, No. 2 , 247-259.

Rao, N. S. V. K. (1998). Vibration analysis and foundation dynamics. Wheeler Publishing, New Delhi, India. 
Rao, N. S. V. K., Das, Y. C. \& Anandakrishnan, M. (1971). Variational approach to beams on elastic foundations. J. Engng. Mech. Div., Am. Soc. Civ. Engrs. 97, No. EM2, 271-294.

Rao, S. N., Ramakrishna, V. G. S. T. \& Rao, M. B. (1998). Influence of rigidity on laterally loaded pile groups in marine clay. J. Geotech. Geoenv. Engng., Am. Soc. Civ. Engrs. 124, No. 6, 542-549.

Reddy, J. N. (2002). Energy principles and variational methods in applied mechanics. John Wiley \& Sons, Inc.

Reddy, A. S. \& Valsangkar, A. J. (1970). Generalized solutions for laterally loaded pile in elastoplastic soil. Soils Fdns. X, No. 3, 66-80.

Reddy, A. S. \& Valsangkar, A. J. (1971). Analysis of a laterally loaded pile in a layered soil by energy method. Acta Technica Academiae Scientiarum Hungaricae, Tomus 70 (1-2), 235-249.

Reese, L. C. (1977). Laterally loaded piles: program documentation. J. Geotech. Engng. Div., Am. Soc. Civ. Engrs. 103, No. GT4, 287-305.

Reese, L. C. (1997). Analysis of laterally loaded piles in weak rock. J. Geotech. Geoenv. Engng., Am. Soc. Civ. Engrs. 123, No. 11, 1010-1017.

Reese, L. C. \& Cox, W. R. (1969). Soil behavior from analysis of tests of uninstrumented piles under lateral loading. Performance of Deep Fdns., ASTM STP 444, 160-176.

Reese, L. C. \& Matlock, H. (1956). Non-dimensional solutions for laterally loaded piles with soil modulus assumed proportional to depth. Proc. $8^{\text {th }}$ Texas Conf. on Soil Mech. Fdn. Engng., Austin, Texas, 1-41.

Reese, J. C. \& Van Impe, W. F. (2001). Single piles and pile groups under lateral loadings. A. A. Balkema, Rotterdam.

Reese, L. C. \& Welch, R. C. (1975). Lateral loading of deep foundations in stiff clay. J. Geotech. Engng. Div., Am. Soc. Civ. Engrs. 101, No. GT7, 633-649.

Reese, L. C., Cox, W. R. \& Koop, F. D. (1974). Analysis of laterally loaded piles in sand. Proc. $6^{\text {th }}$ Offshore Tech. Conf., Houston, Texas, 2, 473-483.

Reese, L. C., Wright, S. G. \& Aurora, R. P. (1984). Analysis of a pile group under lateral loading. Laterally Loaded Deep Fdns., ASTM STP 835, 56-71.

Reese, L. C., Cox, W. R. \& Koop, F. D. (1975). Field testing and analysis of laterally loaded piles in stiff clay. Proc. $7^{\text {th }}$ Offshore Tech. Conf., Houston, Texas, 2, 671-690.

Reese, L. C., Isenhower, W. M. \& Wang, S.-T. (2006). Analysis and design of shallow and deep foundations. John Wiley \& Sons, Inc. 
Rollins, K. M. \& Sparks, A. (2002). Lateral resistance of full-scale pile cap with gravel backfill. J. Geotech. Geoenv. Engng., Am. Soc. Civ. Engrs. 128, No. 9, 711-723.

Rollins, K. M., Peterson, K. T. \&Weaver, T. J. (1998). Lateral load behavior of full-scale pile group in clay. J. Geotech. Geoenv. Engng., Am. Soc. Civ. Engrs. 124, No. 6, 468-478.

Ruesta, P. F. \& Townsend, F. C. (1997). Evaluation of laterally loaded pile group at Roosevelt bridge. J. Geotech. Geoenv. Engng., Am. Soc. Civ. Engrs. 123, No. 12, 1153-1161.

Salgado, R. (2008). The engineering of foundations. The McGraw-Hill Companies, Inc.

Scott, R. F. (1981). Foundation analysis. Prentice-Hall, Inc.

Selvadurai, A. P. S. (1979). Elastic analysis of soil-foundation interaction, Elsevier Scientific Publishing Company.

Sharnouby, B. E. \& Novak, M. (1985). Static and low-frequency response of pile groups. Can. Geotech.J. 22, 79-94.

Shen, W. Y. \& Teh, C. I. (2002). Analysis of laterally loaded pile groups using variational approach. Geotechnique 52, No. 3, 201-208.

Shen, W. Y. \& Teh, C. I. (2004). Analysis of laterally loaded piles in soil with stiffness increasing with depth. J. Geotech. Geoenv. Engng., Am. Soc. Civ. Engrs. 130, No. 8, 878-882.

Shibata, T, Yashima, A., Kimura, M. \& Fukada, H. (1988). Analysis of laterally loaded piles by quasi-three-dimensional finite element method. Proc. $6^{\text {th }}$ Intl. Conf. Numer. Meth. Geomech., Innsbruck, 1051-1058.

Shibuya, S., Tatsuoka, F., Teachavorasinskun, S. Kong, X. J. Abe, F., Kim, Y.-S. \& Park, C.-K. (1992). Elastic deformation properties of geomaterials. Soils Fdns. 32, No. 3, 26-46.

Sogge, R. L. (1981). Laterally loaded pile design. J. Geotech. Engng. Div., Am. Soc. Civ. Engrs 107, No. GT9, 1179-1199.

Stewart, D. P. (2000). User Manual PYGMY. Univ. West. Aust.

Strang, G. (1988). Linear algebra and its applications. Thomson Learning, Inc.

Sun, K. (1993). Static analysis of laterally loaded piles. Proc. $11^{\text {th }}$ Southeast Asian Geotech. Conf., Singapore, 589-594.

Sun, K. (1994a). Laterally loaded piles in elastic media. J. Geotech. Engng., Am. Soc. Civ. Engrs. 120, No. 8, 1324-1344.

Sun, K. (1994b). A numerical method for laterally loaded piles. Comput. Geotech. 16, 263-289. 
Terzaghi, K. (1955). Evaluation of coefficients of subgrade reaction. Geotechnique 5, No. 4, 297-326.

Tomlinson, M. J. (1994). Pile design and construction practice. E \& FN Spon.

Trochanis, A. M., Bielak, J. \& Christiano, P. (1991). Three-dimensional nonlinear study of piles. J. Geotech. Engng., Am. Soc. Civ. Engrs 117, No. 3, 429-447.

Vallabhan, C. V. G. (1999). Validity of the Reese model for pile foundations using variational principles. Anal. Des. Const. Testing Deep Fdns., Geotech. Sp. Publ. 88, Am. Soc. Civ. Engrs., 61-75.

Vallabhan, C. V. G. \& Das, Y. C. (1988). Parametric study of beams on elastic foundations. $J$. Engng. Mech., Am. Soc. Civ. Engrs 114, No. 12, 2072-2082.

Vallabhan, C. V. G. \& Das, Y. C. (1991). Modified Vlasov model for beams on elastic foundations. J. Geotech. Engng., Am. Soc. Civ. Engrs 117, No. 6, 956-966.

Valsangkar, A. J., Rao, N. S. V. K. \& Basudhar, P. K. (1973). Generalized solutions of axially and laterally loaded piles in elasto-plastic soil. Soils Fdns. 13, No. 4, 1-14.

Veruijt, A. \& Kooijman, A. P. (1989). Laterally loaded piles in a layered elastic medium. Geotechnique 39, No. 1, 39-46.

Vesić, A. B. (1961). Bending of beams resting on isotropic elastic solid. J. Engng. Mech. Div., Am. Soc. Civ. Engrs. 87, No. EM2, 35-53.

Viggiani, G. \& Atkinson, J. H. (1995). Stiffness of fine-grained soil at very small strains. Geotechnique 45, No. 2, 249-265.

Vlasov, V. Z. \& Leont'ev, N. N. (1966). Beams, plates and shells on elastic foundations. Israel Program for Scientific Translations, Jerusalem.

Wakai, A, Gose, S. \& Ugai, K. (1999). 3-D elasto-plastic finite element analysis of pile foundations subjected to lateral loading. Soils Fdns. 39, No. 1, 97-111.

Wesselink, B. D., Murff, J. D., Randolph, M. F. Numez, I. L. \& Hyden, A. M. (1988). Analysis of centrifuge model test data from laterally loaded piles in calcareous sand. Engineering for calcareous sediments, 1, Balkema, Rotterdam, 261-170.

Winkler, E. (1867). Die Lehre von der Elasticitaet und Festigkeit, Prag, Dominicus.

Wu, D., Broms, B. B. \& Choa, V. (1998). Design of laterally loaded piles in cohesive soils using p-y curves. Soils Fdns. 38, No. 2, 17-26. 
Xu, K. J. \& Poulos, H. G. (2000). General elastic analysis of piles and pile groups. Int. J. Numer. Anal. Meth. Geomech. 24, 1109-1138.

Yan, L. \& Byrne, P. M. (1992). Lateral pile response to monotonic pile head loading. Can. Geotech. J. 29, 955-970.

Yang, K. \& Liang, R. (2006). Numerical solution for laterally loaded piles in a two-layer soil profile. J. Geotech. Geoenv. Engng., Am. Soc. Civ. Engrs. 132, No. 11, 1436-1443.

Yang, C. C., Lin, S.-S., Juang, C. H. \& Lee, W. F. (2002). Analysis of laterally loaded piles in a two-layered elastic medium. Deep Fdns. 2002 1, Am. Soc. Civ. Engrs., GSP 116, 80-93.

Zhang, L. M. (2003). Behavior of laterally loaded large-section barrettes. J. Geotech. Geoenv. Engng., Am. Soc. Civ. Engrs. 129, No. 7, 639-648.

Zhang, H. H. \& Small, J. C. (2000). Analysis of capped pile groups subjected to horizontal and vertical loads. Comput. Geotech. 26, 1-21.

Zhang, L., McVay, M. C. \& Lai, P. W. (1999a). Centrifuge modelling of laterally loaded single battered piles in sands. Can. Geotech. J. 36, 1074-1084.

Zhang, L., McVay, M. C. \& Lai, P. (1999b). Numerical analysis of laterally loaded $3 \times 3$ to $7 \times 3$ pile groups in sands. J. Geotech. Geoenv. Engng., Am. Soc. Civ. Engrs. 125, No. 11, 936-946.

Zhang, L., Ernst, H. \& Einstein, H. H. (2000). Nonlinear analysis of laterally loaded rocksocketed shafts. J. Geotech. Geoenv. Engng., Am. Soc. Civ. Engrs 126, No. 11, 955-968.

Zhang, L., Silva, F. \& Grismala, R. (2005). Ultimate lateral resistance to piles in cohesionless soils. J. Geotech. Geoenv. Engng., Am. Soc. Civ. Engrs. 131, No. 1, 78-83.

Zhaohua, F. \& Cook, R. D. (1983). Beam elements on two-parameter elastic foundations. $J$. Engng. Mech., Am. Soc. Civ. Engrs. 109, No. 6, 1390-1402. 